\title{
Water dynamics in the rhizosphere How mucilage affects water flow in soils
}

\author{
Dissertation
}

zur Erlangung des mathematisch-naturwissenschaftlichen Doktorgrades

\author{
"Doctor rerum naturalium" \\ der Georg-August-Universität Göttingen \\ im Promotionsprogramm für Agrarwissenschaften \\ der Georg-August University School of Science (GAUSS)
}

vorgelegt von

Eva Kröner

geboren 21.08.1987 in Aachen 

Betreuungsausschuss

Jun. Prof. Dr. Andrea Carminati,

Abteilung für Bodenhydrologie, Georg-August Universität Göttingen

Prof. Dr. Dr. h.c. Hannes Flühler,

Departement Umweltsystemwissenschaften, ETH Zürich

Prof. Dr. Marco Bittelli,

Department of Agricultural Sciences, University of Bologna

Weiterer Betreuer

Dr. Mohsen Zarebanadkouki,

Abteilung für Bodenhydrologie, Georg-August Universität Göttingen

$\underline{\text { Mitglieder der Prüfungskommission }}$

Referent: Jun. Prof. Dr. Andrea Carminati,

Abteilung für Bodenhydrologie, Georg-August Universität Göttingen

Korreferent: Prof. Dr. Dr. h.c. Hannes Flühler,

Departement Umweltsystemwissenschaften, ETH Zürich

2. Korreferent: Prof. Dr. Marco Bittelli,

Department of Agricultural Sciences, University of Bologna

Weitere Mitglieder der Prüfungskommission

Prof. Dr. Yakov Kuzjakov,

Ökopedologie der gemäßigten Zonen, Georg-August Universität Göttingen

Prof. Dr. Kerstin Wiegand,

Ecosystem Modelling, Georg-August Universität Göttingen

Prof. Dr. Heiko Becker,

Zuchtmethodik bei Mais und Raps, Georg-August Universität Göttingen

Tag der mündlichen Prüfung: 10. Februar 2016 



\section{Acknowledgements}

I would like to express my gratitude to my supervisor Andrea Carminati for many great and inspiring ideas, for pointing out this topic of my thesis, for his support of my work and many fruitful discussions.

Next, I would like to thank Marco Bittelli for his encouragement, his help in questions related to soil physics and for guiding me to further scientific topics.

I thank Kurt Roth and Olaf Ippisch for teaching me soil physics and its numerical aspects and for drawing my interest to this exciting research area.

This thesis would not have been possible without the contribution of many scientists. I would like to acknowledge:

- Mohsen Zarebanadkouki, Mutez Ali Ahmed, Maire Holz for the joint work, the help with neutron radiography, many exciting discussions, scientific ideas and a great time - especially during the long days at PSI,

- Nele Richter-Harder, Pascal Benard and Katayoun Ahmadi for inspiring discussions,

- Anders Kästner, Peter Vontobel and Jan Hovind for their support and for providing the opportunity to conduct experiments using neutron radiography,

- Hannes Flühler and Dani Or for scientific hints and inspiration,

- Ingrid Ostermeyer for her support in the laboratory, especially for the huge amounts of mucilage she prepared and that have been essential for this thesis,

- Karin Schmidt for help in the laboratory,

- my office mates Joscha Becker, Kevin Mganga, Silke Hafner, Thomas Splettstößer and the Institute of Soil Science at the University of Göttingen for the nice atmosphere,

- the Soil Science Soccer group.

Finally, I thank my family for supporting me the entire time.

This work has been supported by the Dorothea-Schlözer Stipendium of Göttingen University and the German Research Foundation (DFG) under Project MUCILAGE CA 921/2-1. The Paul Scherrer Institute is acknowledged for the opportunity to monitor water content distributions using neutron radiography. Some numerical problems have been computed on the scientific compute cluster of the University Göttingen (GWDG). I thank people involved in developing the distributed and unified numerics evinronment (DUNE). 


\section{Abstract}

The flow of water from soil to plant roots is affected by the narrow region of soil close to the roots, the so-called rhizosphere. The rhizosphere is influenced by mucilage, a polymeric gel exuded by roots that alters the hydraulic properties of the rhizosphere. When in contact with water mucilage can hold large volumes of water but when dry it turns hydrophobic.

Here we focus on the effect of mucilage on soil hydaulic properties. At first we present experimental and numerical studies describing the rewetting dynamics of dry rhizosphere. Below a certain concentration of mucilage water could flow across the rhizosphere layer, above this concentration the layer turned water repellent. We present an analytical estimation of mucilage concentration at percolation threshold as function of mean soil particle size and bulk soil water potential after irrigation. The estimation was validated with capillary rise experiments.

We developed an effective model describing how mucilage alters soil hydraulic functions, namely (a) swelling and shrinking dynamics of mucilage result in non-equilibrium dynamics between water potential and water content, (b) the presence of mucilage in gel reduces the water potential at a given water content and (c) mucilage is viscous and reduces the soil hydraulic conductivity at a given water content.

In experiments on soil-mucilage mixtures we tested the model and we applied it to simulate observations of past experiments with real plants that show evidences of altered hydraulic dynamics in the rhizosphere.

Attached to this thesis are two studies on heat dissipation from underground electrical power cables. Here hydraulic dynamics similar to those of water flow towards a single root can occur.

\section{Zusammenfassung}

Die Wurzelwasseraufnahme aus dem Boden wird durch die Rhizosphäre beeinflusst. Die Rhizosphäre ist eine dünne Bodenschicht, die sich um Wurzeln herum bildet. Die Rhizosphäre wird durch Mucilage beeinflusst. Mucilage ist ein polymeres Gel, was von Wurzeln abgesondert wird und vor allem die hydraulischen Eigenschaften der Rhizosphäre verändert. Wenn es im Kontakt mit Wasser ist, kann Mucilage große Mengen an Wasser aufnehmen, aber wenn es trocken ist, wird seine Oberfläche hydrophob.

Hier konzentrieren wir uns auf den Effekt von Mucilage auf die hydraulischen Eigen- 
schaften des Bodens. Zunächst präsentieren wir experimentelle und numerische Studien, die die hydraulischen Prozesse in der Rhizosphäre nach der Bewässerung von trockenem Boden beschreiben. Bei Mucilagekonzentrationen, die niedriger als ein gewisser Schwellwert waren, konnte Wasser durch die Rhizosphärenschicht fließen, über dieser Konzentration wurde die Schicht wasserundurchlässig während der ersten Minuten bis zu Stunden nach Bewässerung. We präsentieren eine analytische Abschätzung der Mucilagekonzentration an der Perkolationsschwelle als Funktion von mittlerer Teilchengröße und Bodenwasserpotential nach Bewässerung. Die Abschätzung wurde an Hand von Experimenten des kapillaren Aufstiegs in Bodensäulen validiert.

Wir entwickelten ein effektives Model um zu beschreiben, wir Mucilage die hydraulischen Funktionen des Bodens verändert: (a) Quell- und Trocknungsprozesse von Mucilage resultieren in Nicht-Gleichgewichtsdynamiken zwischen Wassergehalt und Wasserpotential, (b) die Präsenz von Mucilage im Boden reduziert das Wasserpotential bei einem gegebenen Wassergehalt und (c) Mucilage ist viskos und reduziert dadurch die hydraulische Leitfähigkeit des Bodens bei einem gegebenen Wassergehalt.

In Experimenten mit Boden-Mucilage-Mischungen testeten wir das Model und wandten es an, um Beobachtungen von früheren Experimenten mit echten Pflanzen zu simulieren, die veränderte hydraulische Dynamiken in der Rhizophäre zeigen.

Im Anhang dieser Arbeit sind zwei Studien zur Wärmeausbreitung von Erdkabeln. Hier können hydraulische Dynamiken autreten, die dem radialen Wasserfluss zu einer einzelnen Wurzel ähneln. 


\section{Contents}

List of Figures vii

List of Tables $\quad$ xviii

1 Introduction 1

1.1 Topic . . . . . . . . . . . . . . . . . . . . . . . . . . . . . 1

1.2 Outline . . . . . . . . . . . . . . . . . . . . . . 2

1.3 Material and Methods $\ldots \ldots \ldots \ldots \ldots \ldots$

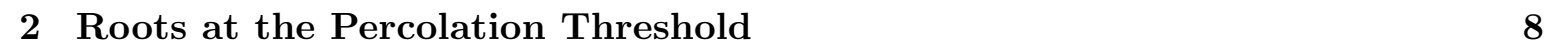

2.1 Introduction . . . . . . . . . . . . . . . . . . . . . . . 8

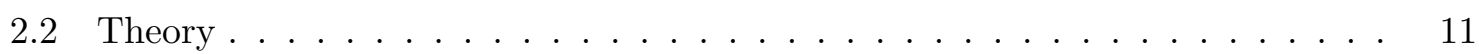

2.3 Measurements . . . . . . . . . . . . . . . . . . . . . . . . . . . 15

2.4 Discussion . . . . . . . . . . . . . . . . . . . . . . . . . . . . . . . . . . . . 17

\begin{tabular}{lll}
\hline 3 & Water percolation through the root-soil interface & 19
\end{tabular}

3.1 Introduction . . . . . . . . . . . . . . . . . . . . . . . . . . . . . . . . 19

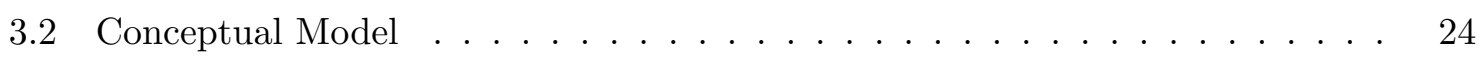

3.3 Material and Methods . . . . . . . . . . . . . . . . . . . . . . 27

3.4 Results and Discussion $\ldots \ldots \ldots \ldots \ldots$

3.5 Conclusions . . . . . . . . . . . . . . . . . . . . . . . . . . 35

4 Drying of mucilage causes water repellency in the rhizosphere of maize: measurements and modelling ${ }^{\star} \quad 36$

4.1 Introduction $\ldots \ldots \ldots \ldots \ldots \ldots \ldots$

4.2 Materials and methods . . . . . . . . . . . . . . . . . . . . . . . . 39

4.3 Conceptual model $\ldots \ldots \ldots \ldots$. . . . . . . . . . . . . . . . 42

4.4 Results and Discussions $\ldots \ldots \ldots \ldots \ldots \ldots$

5 Non-equilibrium water dynamics in the rhizosphere: How mucilage affects

water flow in soils $\quad 53$

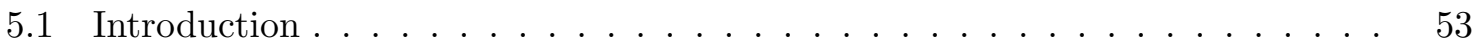

5.2 Material and Methods $\ldots \ldots \ldots \ldots \ldots$. . . . . . . . . . 57

5.2 .1 Model description $\ldots \ldots \ldots \ldots \ldots$. . . . . . . . . . . . 57

5.2 .2 Numerical solution . . . . . . . . . . . . . . . . . . . . . 65 
$5.2 .3 \quad$ Parameter estimation and experimental validation of the model . . . . 66

5.3 Results . . . . . . . . . . . . . . . . . . . . . . . . 71

5.3 .1 Water retention curve . . . . . . . . . . . . . . . . . 71

5.3.2 Hydraulic conductivity as a function of mucilage concentration . . . . 73

$5.3 .3 \quad$ Non-equilibrium monitored with neutron radiography $\ldots . . . . . . .73$

5.4 Discussion . . . . . . . . . . . . . . . . . . . . . . . . . . . . 78

6 Mucilage exudation facilitates root water uptake in dry soils ${ }^{\star} \quad 81$

6.1 Introduction . . . . . . . . . . . . . . . . . . . . . . . . . . 81

6.2 Materials and methods . . . . . . . . . . . . . . . . . . . . . 83

6.3 Results . . . . . . . . . . . . . . . . . . . . . . . . . 90

6.4 Discussion . . . . . . . . . . . . . . . . . . . . . . . . . . 98

7 Simulation of root water uptake under consideration of non-equilibrium $\begin{array}{ll}\text { dynamics in the rhizosphere } & 101\end{array}$

$7.1 \quad$ Introduction . . . . . . . . . . . . . . . . . . . . . . . . . . . . . . . . . . . . 101

7.2 Material and Methods . . . . . . . . . . . . . . . . . . . . . . 103

$7.2 .1 \quad$ Hydraulic properties of the rhizosphere $\ldots \ldots \ldots$

7.2 .2 Implementation of root water uptake model . . . . . . . . . . . . . . 107

$7.2 .3 \quad$ Simulated experiments . . . . . . . . . . . . . . . . . . . 108

7.3 Results . . . . . . . . . . . . . . . . . . . . . . . . . . . . . 110

$7.3 .1 \quad$ Summary of the results $\ldots \ldots \ldots \ldots$. . . . . . . . . 116

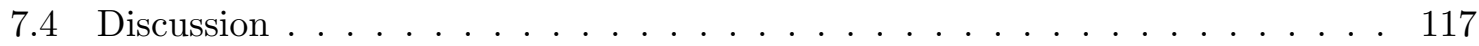

7.5 Supplementary Material $\ldots \ldots \ldots$. . . . . . . . . . . . . . . . . . . . . . 119

\begin{tabular}{llr}
\hline 8 & Conclusions & 124
\end{tabular}

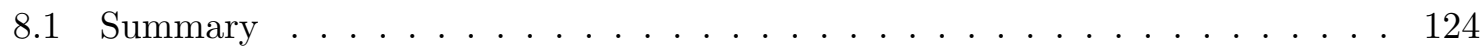

8.2 Application, limitations and outlook . . . . . . . . . . . . . 125

A Numerical simulation of coupled heat, liquid water and water vapor in soils

$\begin{array}{ll}\text { for heat dissipation of underground electrical power cables } & 127\end{array}$

A.1 Introduction . . . . . . . . . . . . . . . . . . . . . . . 127

A.2 Theory . . . . . . . . . . . . . . . . . . 130

A.2.1 Description of coupled model . . . . . . . . . . . . . . . . 130

A.2.2 Atmospheric boundary conditions . . . . . . . . . . . . . . 135 
A.2.3 Boundary conditions at cable surface . . . . . . . . . . . . . 138

A.3 Simulations . . . . . . . . . . . . . . . . . . . . . . . . . . . . 139

A.3.1 Applications . . . . . . . . . . . . . . . . . . . 139

A.3.2 Numerical Implementation . . . . . . . . . . . . . . . . . . . . 146

A.4 Results and Discussion . . . . . . . . . . . . . . . . . . . . . . . . . . . 146

A.4.1 Down-scaled Experiment . . . . . . . . . . . . . . . . . . . 146

A.4.2 Simplified geometry . . . . . . . . . . . . . . . . 147

A.4.3 Simulations under realistic atmospheric conditions . . . . . . . . . . . 152

A.5 Discussion . . . . . . . . . . . . . . . . . . . . . . . . 156

A.6 Nomenclature . . . . . . . . . . . . . . . . . . . . . . . . . . . . . . 159

B Estimation of thermal instabilities in soils for heat dissipation of under$\begin{array}{ll}\text { ground electrical power cables } & 161\end{array}$

B.1 Introduction . . . . . . . . . . . . . . . . . . . . . 161

B.2 Governing equations . . . . . . . . . . . . . . . . . 163

B.3 Order of magnitude estimation $\ldots \ldots \ldots \ldots$. . . . . . . . . 166

B.3.1 Limiting rate of liquid water flow toward the cable . . . . . . . . . . . 167

B.3.2 Vapor flow away from the cable . . . . . . . . . . . . . . . . . . 168

B.3.3 Critical heat dissipation rate . . . . . . . . . . . . . . . . 170

B.4 Numerical simulation . . . . . . . . . . . . . . . . . . . . . . . . . . 170

B.4.1 Parameter functions . . . . . . . . . . . . . . . . . . . . . . 171

B.4.2 Boundary conditions . . . . . . . . . . . . . . . . 172

B.4.3 Critical bulk water potential $\ldots \ldots \ldots \ldots$. . . . . . . . . 172

B.4.4 Implementation . . . . . . . . . . . . . . . . . . . 172

B.5 Results. . . . . . . . . . . . . . . . . . . . . . 173

B.6 Discussion . . . . . . . . . . . . . . . . . . . . . . . . . 177

B.7 Conclusion . . . . . . . . . . . . . . . . . . . . . . 181

B.8 Nomenclature . . . . . . . . . . . . . . . . . . . . . . . . . . . . . . . 182

\begin{tabular}{lr}
\hline References & 183
\end{tabular}

In chapters marked with ${ }^{*}$ I contributed as second author, in the other chapters as first author. 


\section{List of Figures}

$1.140 \%$ of the terrestrial precipitation flows across the rhizosphere - a small layer of soil around roots that is affected by mucilage. Mucilage is a polymeric gel exuded at the tip of plant roots. . . . . . . . . . . . . . 1

1.2 Left: mucilage appears in form of a transparent capsule around the seeds. Right: gel extracted from the seeds. . . . . . . . . . . . . . . . 6

$2.1 \quad$ Water content distribution in the root zone of a young lupine after irrigation \begin{tabular}{l}
\hline as imaged with neutron radiography. The grey values are proportional to the \\
\hline water content (dark=wet). The radiograph shows that the water content in \\
\hline the rhizosphere was markedly drier than in the adjacent bulk soil. The image \\
\hline is taken from the experiments described in Carminati (2013).
\end{tabular}

2.2 Non-equilibrium relation (Eq. 2.1$)$ between rhizosphere water content and \begin{tabular}{|l|}
\hline water potential in the rhizosphere during the rewetting phase (Kroener et al., \\
\hline 2014b). The water content recovers more slowly than the water potential, \\
\hline resulting in a deviation from the equilibrium curve. . . . . . . . . . . . 10
\end{tabular}

2.3 Conceptual model of rewetting of dry rhizosphere. a) Illustration of water content in the rhizosphere during drying and after rewetting; b) rewetting of a \begin{tabular}{|c|}
\hline pore covered with dry mucilage; c) pore network model of bonds covered with \\
\hline
\end{tabular}

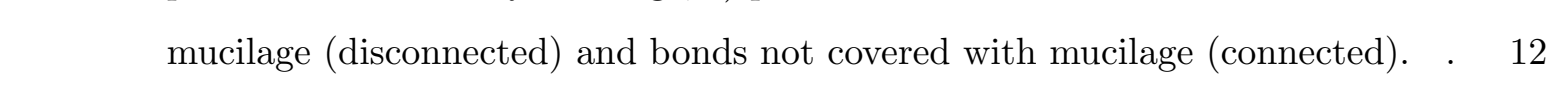

2.4 Bond percolation in a cubic lattice with varying percentage of disconnected \begin{tabular}{|c|}
\hline pores. a) Water filled pores averaged across the sample and for a horizontal \\
\hline
\end{tabular} \begin{tabular}{|c|}
\hline cross section. b) Water filled nodes as a function of disconnected bonds. c) \\
\hline
\end{tabular} Water-filled nodes at the outflow as a function of disconnected bonds. Close to the percolation threshold a small change in the number of disconnected nodes strongly affects the hydraulic behavior. . . . . . . . . . . . . . . . 14

2.5 Capillary rise in soil columns with a small layer of soil mucilage mixture as

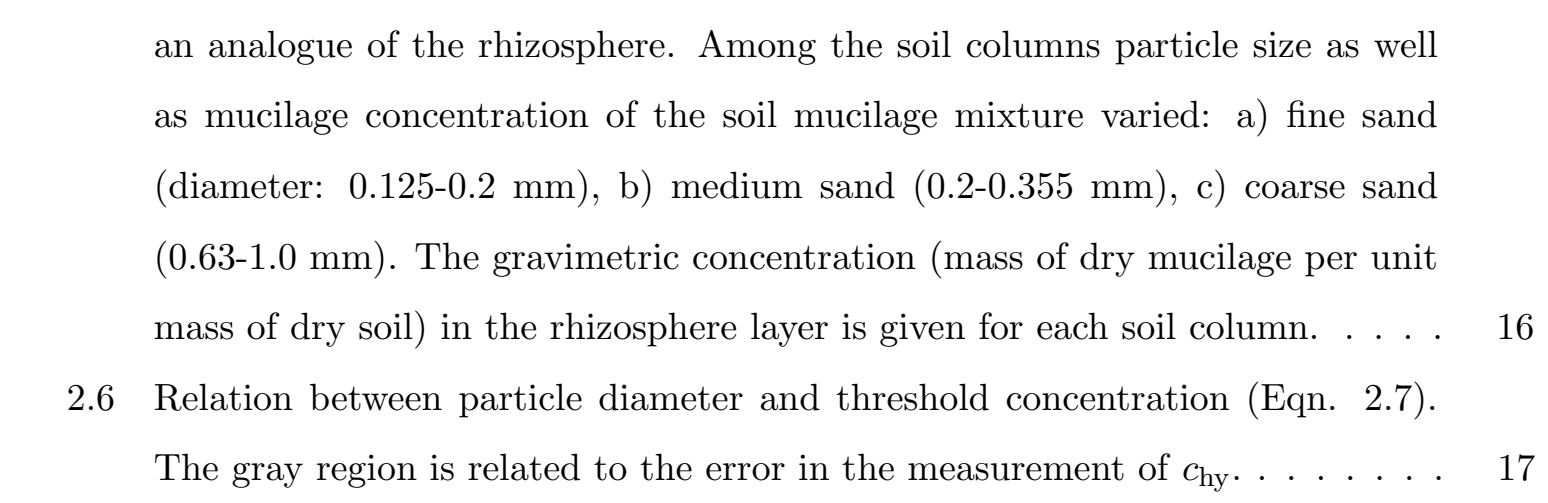


3.1 Neutron radiographs of water distribution around the roots of lupines after irrigation. Left: lupine in a sandy soil; right: lupine in a coarse quartz sand. The samples were rewetted by capillary rise, with the water table at a depth of $15 \mathrm{~cm}$ from the soil surface. The grey values are proportional to the water content (dark=dry, bright=wet). Dry zones are visible around the roots, in particular in the coarse quartz sand (right). . . . . . . . . . . . . . 22

3.2 Illustration of the effect of mucilage on water flow in the rhizosphere. After drying mucilage becomes hydrophobic and impedes the water flow in a fraction of the pores. . . . . . . . . . . . . . . . . . . . . . . . . . 24

$3.3 \quad$ Example of mucilage distribution in the pore-network model. According to the percolation theory, at least $31.17 \%$ of the pores must be conductive to enable the water flow from one side of the system to the opposite side. Eq.(3.1) allows to calculate if a pore of given radius and mucilage concentration $C_{S}$ is wettable at a given matric potential $h . \ldots \ldots \ldots$. . . . . . . . . . 26

3.4 Simulation of water percolation through a soil with varying mucilage concentrations. The simulations show the invasion of water from the left to the right in a soil with pore radius $r=0.1625 \mathrm{~mm}$ at matric potential $h=-2.5 \mathrm{~cm}$.

Near $C_{t h}$ small changes in mucilage concentrations resulted in a large change in water saturation. . . . . . . . . . . . . . . . . . . . . 27

3.5 Neutron radiographs of the capillary rise experiments in fine sand (particle diameter $0.125-0.2 \mathrm{~mm})$, medium sand $(0.2-0.355 \mathrm{~mm})$ and coarse sand $(0.65-$ $1 \mathrm{~mm})$ at varying matric potentials, $\mathrm{h}=-2.5 \mathrm{~cm}, \mathrm{~h}=-6.5 \mathrm{~cm}$ and $\mathrm{h}=-12 \mathrm{~cm}$. Each sample was prepared with a specific amount of mucilage. The threshold mucilage concentrations $C_{t h}$ are indicated with an arrow. . . . . . . . . . . . 29

3.6 Mucilage concentration at the percolation threshold $\left(C_{t h}\right)$ [g of mucilage per g of dry soil] as a function of soil particle diameter for varying matric potentials (h). The lines show the critical mucilage concentrations derived from the porenetwork simulations. The points with error bars show the experimental results for different soil particle diameters. . . . . . . . . . . . . . . . . . . . . 32

$3.7 \quad$ Measured and fitted relations between mucilage concentration per surface area $\left(C_{S}\right)$ and contact angle $(\alpha)$. Contact angles measured with the sessile drop methods are marked with crosses. The dashed line gave the best fit between observed and simulated $C_{t h}$. 
4.1 Brace roots of a 5-week-old maize plant. Roots were kept in plastic tubes filled with water for $24 \mathrm{~h}$ to keep mucilage fully hydrated. . . . . . . . . . . . . 40

4.2 Illustration of the effect of mucilage on water flow in the rhizosphere. This is a $2 \mathrm{D}$ sketch but the model accounts for the $3 \mathrm{D}$ extension of the rhizosphere. After drying mucilage becomes hydrophobic and impedes the water flow in a fraction of the pores. . . . . . . . . . . . . . . . . . 43

4.3 Neutron radiograph of water distribution around the roots of 3-weeks-old maize 30 min after irrigation. The grey values are proportional to the water content (dark=wet, bright=dry). The figure shows that the rhizosphere of most roots appeared brighter than the bulk soil. This shows that maize rhizosphere remained dry after irrigation. . . . . . . . . . . . . . . . . . 45

4.4 Contact angle of the dry maize mucilage on the glass slides. The contact angle was determined with the sessile drop method. . . . . . . . . . . . . . . . . . 46

4.5 Contact angle of dry mucilage collected from maize plants. Mucilage was hydrophobic $\left(\mathrm{CA}>90^{\circ}\right)$ at higher mucilage concentration. The contact angle decreased for lower mucilage concentrations. The blue line shows the fitting of

4.6 Neutron radiographs of the capillary rise experiments in soil with different particle size $(<20,63-200,200-500,360-630,630-1000 \mu \mathrm{m})$. The lower part of each sample was filled with an untreated sandy soil. The second layer was filled with a soil- mucilage layer with a mucilage concentration of $0.5 \% \mathrm{~g}$ of dry mucilage per $\mathrm{g}$ of dry soil. This layer represented the rhizosphere. Then we added another layer of untreated sand. The figure shows that for particles sizes $<200 \mu \mathrm{m}$ water could easily cross the rhizosphere, while for particle sizes $>360$ $\mu \mathrm{m}$ above the critical concentration water could no longer percolate through it. 48

4.7 Maize mucilage concentration at the percolation threshold (g of mucilage per $\mathrm{cm}^{3}$ of dry soil) as a function of soil particle diameter for varying matric potentials $(h)$. The figure shows the critical maize mucilage concentrations derived from the pore-network simulations . . . . . . . . . . . . . . . . . . 49 
$5.1 \quad$ Neutron radiography of soil moisture distribution around the roots of lupines. The gray values are proportional to water content (dark means wet). The upper picture was taken during drying, the lower picture was taken 10 hours after irrigation. The radiographs show that the water content in the rhizosphere is markedly different from that in the adjacent bulk soil (adapted from Figure 3 in Carminati and Vetterlein $[(2013)$ with kind permission of Oxford University Press). . . . . . . . . . . . . . . . . . . . . 55

5.2 Schematic of the physical system simplified into a one dimensional radial geometry. a) Sketch of mucilage distribution in the pore space around a root. b Radial distribution of non-mucilage-filled pore ratio $R$. c) Dynamic water con\begin{tabular}{|c|}
\hline tent change during drying as a function of distance to root. d) Water content \\
\hline
\end{tabular} change during re-wetting as function of distance to root. . . . . . . . . . . 58

5.3 Illustration of a drying cycle in the rhizosphere. Near the root the drying process is strongly influenced by the presence of mucilage in the pore space: its gel-like properties induce a delay in water content changes after changes in water potential. Far from the root, where most of the pores are not filled with mucilage, the water content follows the changes in water potential. . . . . . . 59

5.4 Dynamic non-equilibrium in the water retention curve of the rhizosphere. Dur\begin{tabular}{l}
\hline ing rewetting/drying cycles we imposed that the water potential increases/decreases \\
\hline exponentially over time. The corresponding water content has been calcu- \\
\hline lated with Eq (5.3). Fast/slow means the applied water potential changes \\
\hline fast/slowly. Here, it is assumed that all pores are filled with mucilage $(R=0)$. 60
\end{tabular}

5.5 a) The water potential increases from a high negative value to a value close to zero. The corresponding water content has been calculated with Eq (5.3): Far from the root $(R=1)$ the change in water content occurs immediately. When mucilage concentration increases $(R=0.5)$ the immediate change in water content occurs in half of the pore fraction, while the remaining half of the pore space reacts with a time delay. At the root surface $(R=0)$ there is no immediate change in water content. In this illustration the possibly different equilibrium water holding capacity of mucilage has not been considered. b) The relation between soil matric potential and water content is illustrated during drying/rewetting (i.e. the applied water potential decreases/increases)

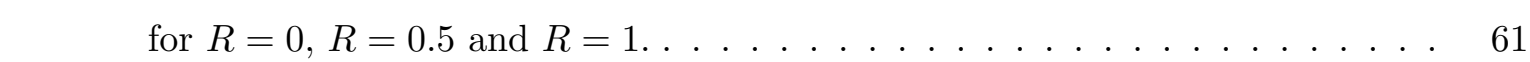


5.6 Illustration of rhizosphere wetting. Close to the root there is a long time delay in water content change. Far from the root the water content changes at the same time as the water potential. Hydrophobicity of mucilage under dry conditions prevents the immediate re-wetting of the pore space when the surface of surrounding soil particles is covered with gel. . . . . . . . . . . . .

5.7 Effect of the osmotic pressure in the mucilage on the equilibrium water reten-

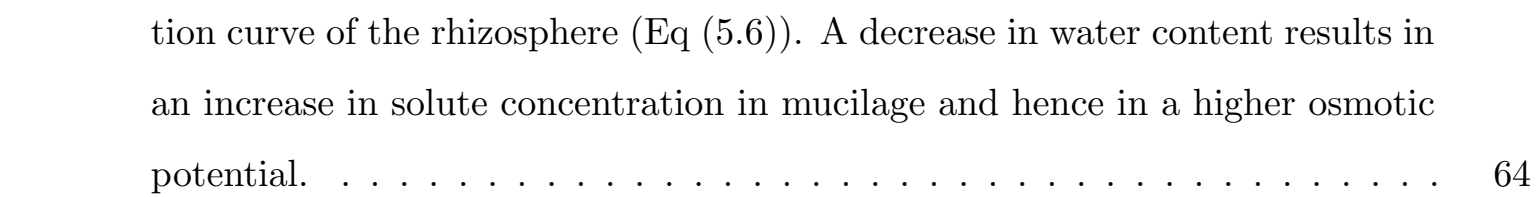

5.8 Relation between viscosity and concentration of a polymeric solution for dilute and concentrated regime. . . . . . . . . . . . . . . . . . 64

5.9 Measured and fitted water retention curve for a treated and untreated sandy soil. Measurements as well as fitted lines according to the parameters of Table 15.1land Table|5.2 are shown. . . . . . . . . . . . . . . . . . . 71

5.10 a) Saturated hydraulic conductivity as a function of mucilage concentration in a sandy soil. Parameters are listed in Table 5.3 . b) Relative viscosity as function of mucilage concentration in water, derived from soil hydraulic conductivity measurements using Eq (5.8). . . . . . . . . . . . . . . . . 72

5.11 a) Water content distribution during wetting as measured with neutron radiography. Zone 1: untreated soil, zone 2: soil mixed with mucilage, zone 3: untreated soil. b) Average water content in the three zones over time. The measured points were obtained from neutron radiographs. The experiment was fitted using the parameters of Table|5.4 $\ldots \ldots \ldots$. . . . . . . . 74

5.12 a) Water content in soil during drying measured by neutron radiography. Zone

1: untreated soil, zone 2: treated soil, zone 3: untreated soil. b) Water content of the three zones over time. The figure shows measurement and simulation based on parameters of Table 5.4 . . . . . . . . . . . . . . . 75

5.13 Sensitivity analysis of the model to the saturated hydraulic conductivity of \begin{tabular}{|l|}
\hline untreated soil $K_{\text {sat }}$ and to the relaxation time parameter $\tau_{0}$ during the wetting \\
\hline (a,b) and drying (c,d) experiments. Continuous lines correspond to the best
\end{tabular} fit, dotted/dashed lines correspond to simulations where either $K_{\text {sat }}$ or $\tau_{0}$ was multiplied/divided by a factor of five. . . . . . . . . . . . . . . 77

$6.1 \quad$ The experimental set-up for the root pressure probe experiment. . . . . . . . 86 
6.2 Effect of mucilage on the soil water retention curve. The figure shows that the soil mixed with mucilage retained more water than the control soil at any given water potential. The data points are averages of three replications. The error bars indicate the standard deviation of the measurements. The water

retention curve of the soil without mucilage is fitted with the Brooks-Corey model (solid line). The data of soil with mucilage are fitted with Eqn.(6.5). The fitted parameters are presented in Table (6.1). . . . . . . . . . . . . . 90

6.3 Change in the volumetric water content after injection of mucilage and water. The soil had an initial water content of $0.04 \mathrm{~cm}^{3} \mathrm{~cm}^{-3}$. The figure shows that the soil is drained much more slowly after mucilage injection than after water injection. The experiments were fitted solving the modified Richards' equation

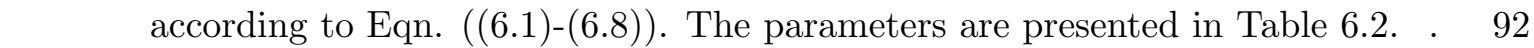

6.4 Pressure relaxation curves during several injection and suction pulses with artificial roots covered and not covered with mucilage. (a) Pressure curve for a root not covered with mucilage. After we placed the artificial roots in soil we covered them with additional dry soil to achieve a good contact between root and soil. Due to the high water tension in the newly added soil, the roo pressure dropped. When the pressure reached a constant value, we applied a \begin{tabular}{|c|}
\hline series of injection and suction pulses over a period of $5 \mathrm{~h}$. The curves were \\
\hline
\end{tabular} fitted by solving the Richards' equation in radial coordinates. Pressure curves for an artificial root covered with mucilage over (b) 0-5 h, (c) 5-10 h and

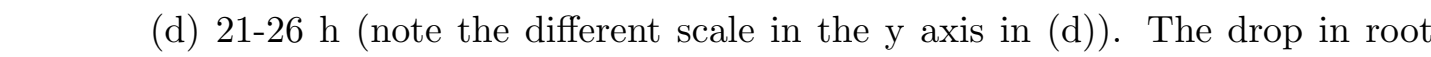
pressure after placing the root in the soil was smaller and the pressure reached equilibrium faster. The pressure relaxations in roots covered with mucilage \begin{tabular}{|c|}
\hline were initially much faster and then they became slower over time. The pressure \\
\hline
\end{tabular} \begin{tabular}{|c|}
\hline relaxation curves were fitted solving Richards' equation modified according to \\
\hline
\end{tabular} \begin{tabular}{|c|c|c|c|}
\hline Eqns ((6.1)-(6.8)). The fitting parameters are shown in Table $|6.2|$ & $\ldots$ \\
\hline
\end{tabular}

6.5 Pressure relaxation curves with artificial roots covered and not with mucilage

\begin{tabular}{l}
\hline at different times. After mucilage application, the pressure relaxation was \\
\hline much quicker than in the soil without mucilage. After 24 hours, the relaxation \\
\hline times became similar. This was caused by the drainage of mucilage over time. \\
\hline The data are normalized between zero and one for comparison. . . . . . . . . . 996
\end{tabular}


6.6 Evolution of the hydraulic conductivity in the soil next to the root for both cases, with and without mucilage. The figure shows that in both cases the hydraulic conductivity decreased after placing the root in soil. This was caused by the outflow of water from the root until the pressure reached equilibrium with the soil. Mucilage increased the hydraulic conductivity of the soil next to the root for the measured 24 hours. During the first 5 hours, the conductivity was increased of a factor of 10 times, which decreased to a factor of 2 after 24 hours. The decreasing differences over time were caused by mucilage drying. . 97

7.1 Water content distribution shortly after irrigation. The light grey boxes indicate the roots that correspond to the stage of the roots in the simulated experiments. As an illustration we used a lupine for this figure. Note that not all the simulated experiments are based on lupines. . . . . . . . . . . . . . . . 104

7.2 (a) Estimated hydraulic conductivity of the rhizosphere after placing mucilage respectively water in a small region around the root [adapted from Ahmed et al. (2014), Fig. 6]. The data have been obtained by fitting the measured \begin{tabular}{|c|}
\hline root pressure of the artificial root. (b) Estimated hydraulic conductivity of \\
\hline
\end{tabular} bulk soil and in the rhizosphere at root surface both during the simulation and under equilibrium conditions. . . . . . . . . . . . . . . . . . . 111

7.3 Top: evolution of average water content in rhizosphere and bulk soil during slow drying and subsequent rewetting for a young (a) and an old lupine roo (b). Bottom: simulated hydraulic conductivity and equilibrium hydraulic conductivity for young (c) and old (d) rhizosphere as function of water potential. Also the bulk soil hydraulic conductivity is shown. . . . . . . . . . . . . . . 113

7.4 (a) Simulated and measured (Zarebanadkouki et al., 2015) evolution of water content as function of distance to the root surface of a lupine during drying and after irrigation. (b) Simulated hydraulic conductivity and equilibrium hydraulic conductivity of the rhizosphere at the root surface as function of water potential. Also the bulk soil hydraulic conductivity is shown. . . . . . . 114 
7.5 (a) Relation between water potential in the leaf xylem and transpiration rate. The dotted line shows the measured data taken from Passioura|(1980), the continuous line shows the result of a simulation under the assumption that the rhizosphere has the same hydraulic properties as the bulk soil, and the dashed line shows the results of a simulation using our model. (b) Hydraulic conductivity as function of water potential. $\ldots \ldots \ldots \ldots$

7.6 Transpiration rate and simulated water content in the rhizosphere at the root \begin{tabular}{|c|}
\hline surface for run E of the experiment of \\
\hline Passioura $[(1980)$. The non-equilibrium \\
\hline concept in the rhizosphere means that at the time when transpiration is highest
\end{tabular} concept in the rhizosphere means that at the time when transpiration is highest water content has not yet reached its lowest value. . . . . . . . . . . . . 116

7.7 Boundary flux used for simulating the experiment described in Carminati (2012). It is obtained from the data of the weight of the sample, see Fig. 2 in Carminati $[2012) . \ldots \ldots \ldots \ldots$. . . . . . . . . . . . . . 120

7.8 Hydraulic properties of the bulk soil used in Passioura (1980). The points show the measured values taken from Passioura $(1980)$ and the lines are the fitted hydraulic functions. . . . . . . . . . . . . . . . . . . . . . . . . . . . . 121

7.9 Transpiration rate versus time for the simulation of the experiment of Passioura (1980). It has been assumed that the transpiration rate increases in each step exponentially to the new transpiration rate and that the time interval of each step is 2000 s. . . . . . . . . . . . . . . . . . . . . . . 123

A.1 Water retention curve and hydraulic conductivity function for two materials that have been used in the simulations, see table|A.1] . . . . . . . . . . . 132

A.2 Thermal conductivity as a function of water content at a temperature of 25 ${ }^{\circ} \mathrm{C}$ for the parameters of table $\mid$ A.1 $\ldots \ldots \ldots$. . . . . . . . . . . . . 135

A.3 Shape of the domain and spatial discretization used in the simulations: a down-scaled experiment b) simplified cylindrical geometry with realistic extensions c) realistic situation with a local grid refinement depending on the scenario: i) concrete slab, ii) hydraulic barrier of v-shape and iii) silt layer. . 140

A.4 Top: Dimensions of experimental set-up. Units are [m]. Bottom: The position of the installed thermocouples (filled circles in the cross sections) and the position of the cable (dark line) within the soil-box. Numbers are the corresponding coordinates, where $x=0$ is in the middle of the box, $y=0$ at the front of the box and $z=0$ at the top. Units are $[\mathrm{m}] . \ldots \ldots \ldots 141$ 
A.5 Weather conditions at the upper boundary. Measured temperature and precipitation and smoothed function of both are shown. They have been smoothed by a convolution with a Gaussian function. The standard deviations of the used Gaussian functions were $6 \mathrm{~d}$ for the temperature plot and $35 \mathrm{~h}$ for the precipitation plot. . . . . . . . . . . . . . . . . . . . . . . . . . 144

A.6 Temporal evolution of the cable's temperature during the 4-day measurement \begin{tabular}{|l|}
\hline period for 4 different runs. For each of them measured data (continuous line) \\
\hline and simulated based on the coupled program (dashed line) and based on the \\
\hline assumptions of a constant thermal resistivity of $0.9 \mathrm{mC} / \mathrm{W}$ (dotted line) are \\
\hline plotted.
\end{tabular} plotted. . . . . . . . . . . . . . . . . . . 147

A.7 Spatial distribution of temperature at the end of run 1. Empty symbols correspond to the measured temperature, black symbols with a continuous line to the simulated data based on the coupled model and empty symbols with dashed line to the simulated data based on the assumption of a constant ther-

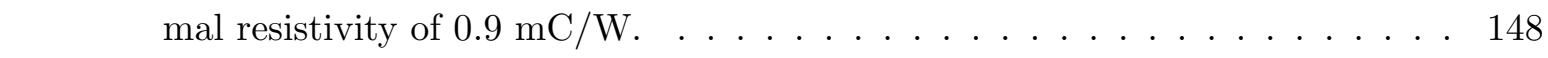

A.8 Evolution of temperature (top) and matric potential at the cable bottom) during the first 10 days under different initial conditions of water potential ranging from $\psi=-0.7 \mathrm{~J} \mathrm{~kg}^{-1}$ to $\psi=-14 \mathrm{~J} \mathrm{~kg}^{-1}$. . . . . . . . . . . . 149

A.9 Radial temperature (top) and radial water content (bottom) distribution after 10 days for different initial water potentials. . . . . . . . . . . . . . . 151

A.10 Simplified geometry simulation: Cable's temperature after 10 days related to initial water content . . . . . . . . . . . . . . . . . . 152

A.11 Thermal energy exchange at the soil surface separated into: sensible heat, latent heat, global solar radiation (measured), radiation reflected by the soil \begin{tabular}{|c|}
\hline surface, long wave radiation from the atmosphere and long wave radiation \\
\hline
\end{tabular} emitted from the soil surface . . . . . . . . . . . . . . . . 153

A.12 Temperature at cable surface versus time. (The temperatures of run A and run B are so close that they can only be distinguished after zooming in.)] . . . 154

A.13 Distribution of temperature and water content at day 150 for the simulated cases (i), (ii) and (iii). . . . . . . . . . . . . . . . . 155

A.14 Water content at cable surface related to time . . . . . . . . . . . . . 156 
A.15 Top: Cable's temperature for run D and smoothed measured air temperature. The smoothed line is also shown in figure|A.5|but here it is shifted forward by 50 days. Bottom: Negative change of the cable's temperature $-d T / d t$ for run A and smoothed precipitation. The smoothed curve is the same as the one shown in Fig $\mid$ A.5 5 but it is now shifted forward by 3.15 days. . . . . . . . . . . 157

B.1 Energy and mass balance for heat dissipation in a) wet soil and b) dry soil. The thickness of the arrows indicates the quantitative contribution of latent heat flux $q_{\mathrm{hL}}$, sensitive heat flux $q_{\mathrm{hC}}$, heat dissipation from the cable $q_{\mathrm{C}}$, water vapor flux driven by a gradient in water potential $q_{\mathrm{vP}}$, liquid water flux $q_{\mathrm{lw}}$, and water vapor flux driven by a gradient in temperature $q_{\mathrm{vT}}$ to the overall energy and mass budget. . . . . . . . . . . . . . . . . . . . . 164

\begin{tabular}{|ll|l|l|}
\hline B.2 & Limiting water flux $q_{\mathrm{lw}}^{\text {lim }}$ as function of bulk soil water potential (Eq. & B.12) for \\
\hline
\end{tabular} typical soil types using the parameters of Tab. B.1 . . . . . . . . . . . . . . . 168

B.3 Thermal conductivity as function of water potential (Eq. (B.16)) obtained as best fit to the data set from McInnes (1981). . . . . . . . . . . . . . . 169

\begin{tabular}{|lll|l|l|}
\hline B.4 Vapor flux $q_{\mathrm{v}}$ as function of bulk soil water potential (Eq. & B.15) for typical \\
\hline
\end{tabular} soil types (Tab. $\mid$ B.1) assuming a cable heat dissipation rate of $q_{\mathrm{C}}=100 \mathrm{~W} \mathrm{~m}^{-1}$. 170

B.5 Slope of saturation vapor concentration (Eq. $\mid$ B.201. . . . . . . . . . . . . . 171

B.6 Relation between bulk soil water potential and cable heat dissipation rate for \begin{tabular}{|r|ll}
\hline various soil types (Tab. & B.1) at the critical point where soil turns thermally \\
\hline
\end{tabular} unstable. The results of the numerical simulations are illustrated as linespoints.

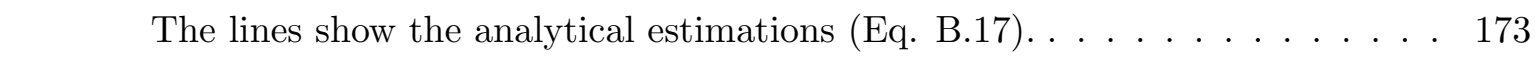

B.7 Radial solution of temperature, water potential, and water content for a sandy soil (top) and a clay soil (bottom) at the critical point. Top: Note the differ\begin{tabular}{|c|}
\hline ent order of magnitude in the radial solution of the water potential for sand \\
\hline
\end{tabular} compared to the clay, as well as the different range of water contents for the two soil types. . . . . . . . . . . . . . . . . . . . 175

B.8 Soil temperature and soil water content at the cable surface as function of heat

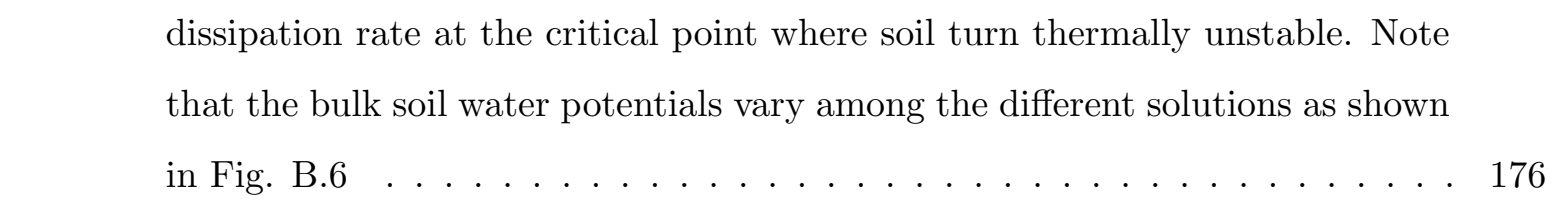


B.9 Conductive heat flow, temperature driven vapor flow, water flow, and water potential driven vapor flow at the critical bulk soil water potential where soil at the cable turns thermally unstable. Solutions are shown for a sandy soil (top) and a clay soil (bottom) and for various cable heat dissipation rates $\left(q_{\mathrm{C}}=20\right.$, 80 and $\left.100 \mathrm{~W} \mathrm{~m}^{-1}\right)$. Note that the scale for the water potential driven vapor flow is logarithmic. . . . . . . . . . . . . . . . . . . . 178

B.10 Radial solution of temperature, water potential, water content, conductive heat flow, temperature driven vapor flow, liquid water flow, and water potential driven vapor flow for a sandy soil for conditions beyond the critical point of thermal instability. They have been obtained by replacing the termination criterion (Eq. (B.23)) of the numerical solver by the condition that the water potential at the cable surface get smaller than $-3 \mathrm{e}-4 \mathrm{~J} \mathrm{~kg}^{-1}$. . . . . . . . . . . 179

B.11 Evaporation and condensation derived from the solutions presented in Fig. $\mid$ B.10! . . . . . . . . . . . . . . . . . . . . . . 180 


\section{List of Tables}

$3.1 \quad$ Soil particle diameters and estimated average surface area $A$ assuming a cubic

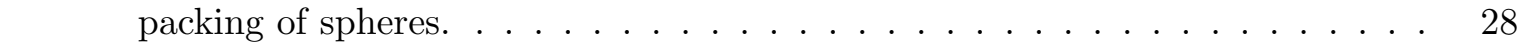

5.1 Brooks-Corey parameters fitted to the measured water retention curve of the

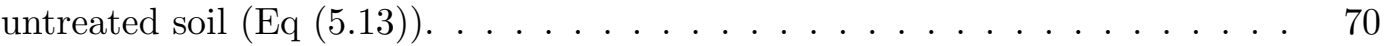

$5.2 \quad$ Parameters describing the increased water holding capacity caused by mucilage (Eq (5.6p). Fit 1 is the best fit, fit 2 is the best fit under the constraint of $\beta=1$ and fit 3 is the best fit for the measured values with $\psi>-1000 \mathrm{hPa}$

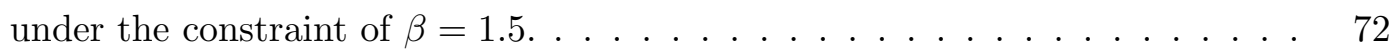

5.3 Parameterization of the reduced relative viscosity (Eq (5.8)). Three parametersets are provided: One for the best fit to the measurements and two modified parameter sets to illustrate the sensitivity towards different parameters. . . . 73

5.4 Parameters to describe the relaxation process of soil-mucilage mixture (Eq (5.4)). The parameters have been fitted to the drying and wetting process measured with neutron radiography. . . . . . . . . . . . . . . . . 73

5.5 Mualem-Brooks-Corey parameters of the unsaturated hydraulic conductivity of the untreated soil $(\mathrm{Eq}(5.14)) . \quad K_{\text {sat }}$ was measured, $L$ was fitted to the wetting and drying experiments observed with neutron radiography. . . . . . 76

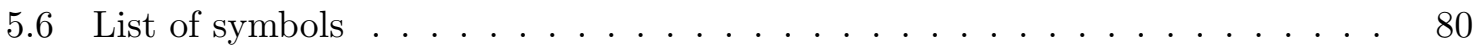

6.1 Brooks-Corey parameters of the soil water retention curve. The parameters were obtained by fitting the pressure plate apparatus measurements. The retention curve of the soil-mucilage mixture is parameterized according to Eqn. $(6.4)$. . . . . . . . . . . . . . . . . . . . . 91

6.2 Parameters of the non-equilibrium model (Eqn.(6.1)). The data are obtained from fitting the root pressure probe measurements and the drying of mucilage with the non-equilibrium model (Eqn.(6.1)). . . . . . . . . . . . . . . 91

7.1 Parameter set obtained by fitting the simulated results to the experimental data. (Unit of $\mathrm{c}$ is $\left.\left.\left[\mathrm{g} \mathrm{cm}^{-3}\right]\right)\right] \ldots \ldots \ldots$. . . . . . . . . . . . 110

7.2 Hydraulic parameters used for the simulation. The parameters have been taken from Carminati $[(2012)$. To account for the low bulk soil water contents measured with neutron radiography, the residual water content has been modified

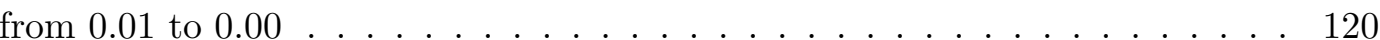


7.3 $\quad$ Parameter set for the hydraulic properties of the bulk soil used by Passioura (1980). The parameters have been obtained by least-square fitting to the data points of water retention and conductivity (Fig. 7.8) . . . . . . . . . . . . 121

A.1 Hydraulic properties of the soil types used in the simulation of the downscaled experiment. Values for $\psi_{e}, b, K_{s}$ and clay content are from Campbell and Norman $\left[(1998)\right.$, the bulk densities $\rho_{b}$ have been measured by de Lieto Vollaro

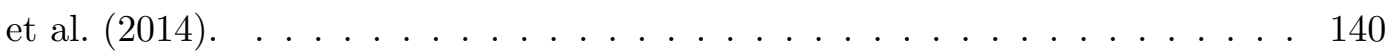

A.2 Hydraulic parameters of the different materials used in Run B-D of the weather study . . . . . . . . . . . . . . . . . . . . . . . 145

A.3 Generation of cable energy at the cable, ambient air temperature and temperature at the cable after 4 days obtained by: experiment, simulation based on coupling of liquid water, vapor and heat and simulation based on the recommended value for thermal resistivity of $0.9 \mathrm{mC} / \mathrm{W} . \quad \ldots \ldots \ldots$

A.4 Average contribution of each component to the energy exchange at the upper surface. Units are $\left[\mathrm{Wm}^{-2}\right] \ldots \ldots \ldots \ldots$. . . . . . . . . . . 152

A.5 Average temperature $T_{\mathrm{av}}$, variation as an indicator for the oscillation of the cable temperature and the average difference between daily maximum and minimum cable temperature. . . . . . . . . . . . . . . . . . . 153

A.6 List of Latin Symbols (A-T) . . . . . . . . . . . . . . . . . . . . . . . . 159

A.7 List of Latin Symbols (U-Z) $\ldots \ldots \ldots$

A.8 List of Greek Symbols ～. . . . . . . . . . . . . . . . . . . . . . . . . 160

B.1 Hydraulic properties of soils as a function of soil texture . . . . . . . . . . . 167

B.2 List of symbols . . . . . . . . . . . . . . . . . . . . . . . . 182 



\section{Introduction}

\section{$1.1 \quad$ Topic}

Water scarcity, desertification and drought together with an increasing population are among the major challenges we are facing in this century - one of the biggest limitations of world wide food production is the availability of fresh water. The current agricultural production, however, is mainly based on resource-intensive farming systems and relies on high availability of water and fertilizers (Lynch and Brown, 2012).

A proper understanding of how plants take up water from soil can help to increase plant drought tolerance and to optimize irrigation techniques. All water that flows through the soilplant-atmosphere continuum - which is around $40 \%$ of the terrestrial precipitation Bengough, 2012 ) - flows across a layer of soil around roots, the so-called rhizosphere (Fig. 1.1). The rhizosphere is actively modified by root growth and root exudates and it is the region of complex and dynamic interactions between roots, bacteria and soil affect the capacity of plants to take up water. It is well known that this region differs in its biological, chemical and physical properties from those of the adjacent bulk soil (Hinsinger et al., 2009). Although this layer has an extension of only some millimeters it is a hydrological hotspot that strongly affects water flow from soil to plant roots. Sposito (2013) suggested that rhizosphere hydraulic processes are essential for a sustainable use of soil-water resources.

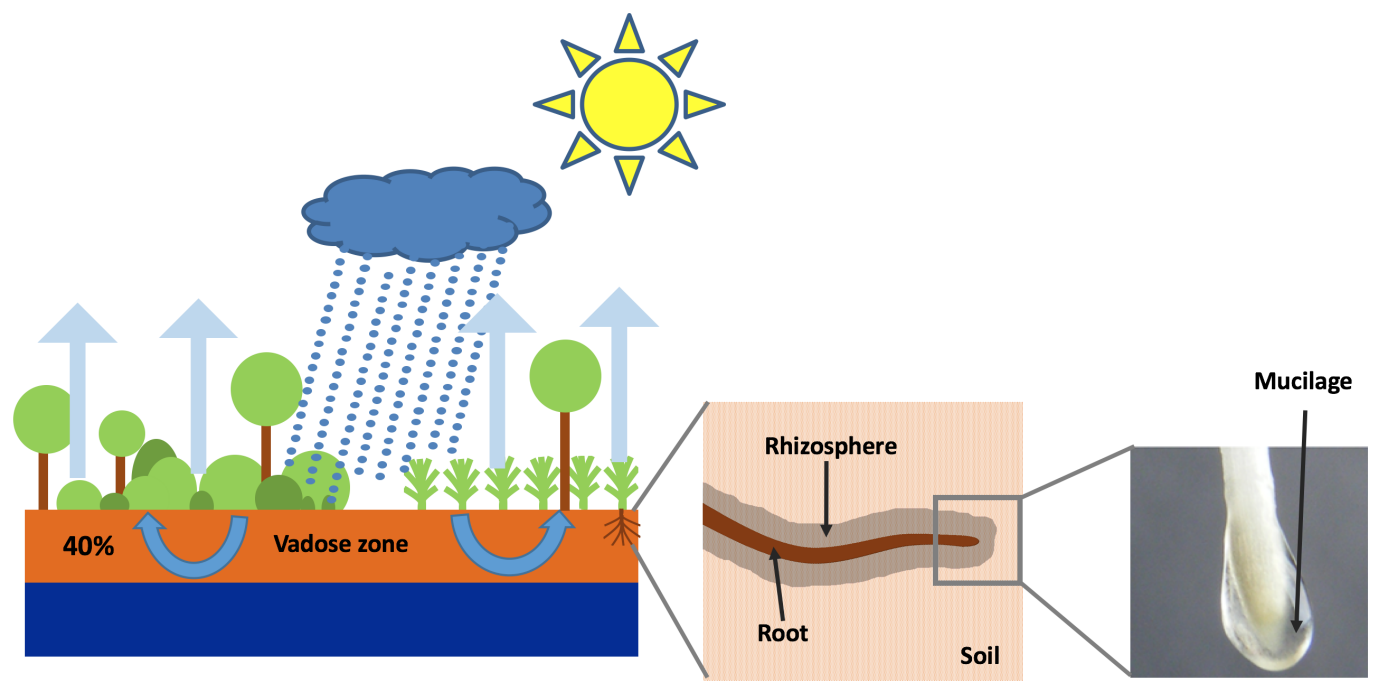

Figure 1.1: $40 \%$ of the terrestrial precipitation flows across the rhizosphere - a small layer of soil around roots that is affected by mucilage. Mucilage is a polymeric gel exuded at the tip of plant roots. 
In my thesis I focus on this region - or more preciously on how its hydraulic properties are affected by mucilage. Mucilage is a gel exuded by roots which absorbs large volumes of water (McCully and Boyer, 1997), but becomes hydrophobic when dry (Moradi et al., 2012).

Our starting-point are recent studies reporting unexpected and puzzling hydraulic dynamics observed in the rhizosphere that could not have been explained under the assumption of homogeneous hydraulic properties in rhizosphere and bulk soil: for drying experiments Carminati et al. (2010) reported a higher water content in the rhizosphere than in the adjacent bulk soil. The assumption of homogeneous hydraulic properties, however, would result in a decrease of water towards the root since water flow is driven by a decrease in water potential and flows from bulk soil to the root. After irrigation of a plant showing first wilting symptoms Carminati et al. (2010) found the water content distribution being reversed: while bulk soil rewetted quickly for the rhizosphere water content it took up to two days to recover. (Zarebanadkouki and Carminati, 2014) found that this lower water content is associated with a root water uptake being reduced by 4-8 times. Moradi et al. (2012) suggested that this water repellency of dry rhizosphere is caused by mucilage that turns hydrophobic when dry. In another study Passioura (1980) measured water potential in the xylem of wheat plants during cycles of increasing and decreasing transpiration rates and reported a puzzling hysteresis and an additional resistance at decreasing transpiration rates that could not have been explained under the assumption of homogeneous hydraulic properties..

The objective of the thesis was to properly understand and describe the hydraulic dynamics in the rhizosphere - more preciously how mucilage alters soil hydraulic dynamics. We combined experiments and numerical modeling to quantitatively describe hydraulic processes on the pore scale as well as their implications on the Darcy scale.

I applied the numerical methods I learned to simulate root water uptake also to other problems: studies on heat dissipation from underground cables (discussed in the Appendix $\mathrm{A}$ and $\mathrm{B}$ and a mathematical study on Numerical approximation of level set power mean curvature flow (Kröner et al., submitted 2015) which is not presented in this thesis.

\subsection{Outline}

The thesis is organized as follows: Chapter 2, 4 focus on rewetting of dry rhizosphere and apply pore network models to derive macroscopic properties from physical relations on the pore scale. In Chapter 5 5 I present and apply a model describing hydraulic dynamics of the rhizosphere on the Darcy scale. In the appendix $\mathrm{A}-\mathrm{B}$ I present studies about heat dissipation 
from electrical underground cables.

Chapter 2 is taken from the article Roots at the Percolation Threshold by Kroener et al. (2015) published in Physical Review E. We present a percolation model to describe rewetting of dry rhizosphere. We found that at a critical mucilage concentration the rhizosphere becomes impermeable. The critical mucilage concentration depends on the radius of the soil particle size. Capillary rise experiments monitored with neutron radiography proved that for concentrations below the critical mucilage concentration water could easily cross the rhizosphere, while above the critical concentration water could no longer percolate through it.

Chapter 3 is taken from the article Water percolation through the root-soil interface by Benard et al. (2015) published in Advances in Water Resources. In this study we extended experiment and theory of the previous study to the more general unsaturated case: at the pore scale we used the Young-Laplace equation to estimate the water potential necessary to rewet a pore as function of contact angle and pore size. A percolation model together with measurements of contact angle as function of mucilage concentration give an estimation for the macroscopic relation between mucilage concentration in soil, mean soil particle size and water potential at percolation threshold. Capillary rise experiments confirmed the estimation obtained from the percolation model.

Chapter 4 is taken from the article Drying of mucilage causes water repellency in the rhizosphere of maize: measurements and modelling by Ahmed et al. (2015c) ${ }^{2}$ published in Plant and Soil. Here the rhizosphere water repellency was shown for a real maize-root system, we found an analytical estimation for the percolation threshold and confirmed it with capillary rise experiments in soil columns prepared with a layer of soil mixed with mucilage collected from maize roots.

Chapter 5 is taken from the article Non-equilibrium water dynamics in the rhizosphere: How mucilage affects water flow in soils by Kroener et al. (2014b) published in Water Resources Research. Here we present a model that describes how a certain concentration of mucilage may affect the soil hydraulic functions: namely (a) swelling and shrinking of mucilage results in a non-equilibrium relation between water content and water potential, (b) mucilage reduces the water potential at a given water content, (c) mucilage is viscous and re-

\footnotetext{
${ }^{1}$ E.K. was responsible for the capilary rise experiments and contributed to the extension of the theoretical concept from the saturated case to the unsaturated case.

${ }^{2}$ E.K. developed the analytical estimation of the percolation threshold and partly prepared the capillary rise experiment.
} 
duces the hydraulic conductivity at a given water content. The model is tested experimentally on soil-mucilage mixtures.

Chapter 6 is taken from the article Mucilage exudation facilitates root water uptake in dry soils by Ahmed et al. (2014) $)^{3}$ published in Functional Plant Biology. In this study we measured the hydraulic conductivity of the soil around an artificial root (suction cup) with the root pressure probe technique combined with numerical modeling. For the simulations we applied the model of Chapter 5. We showed that under dry soil conditions exudation of young mucilage increases the soil hydraulic conductivity.

Chapter 7 is taken from the manuscript Simulation of root water uptake under consideration of non-equilibrium dynamics in the rhizosphere by Kroener et al. (submitted 2016) submitted to Water Resources Research. Here we combined the model from Chapter 5 with the Richards equation to simulate root water uptake of four experiments from the literature that show altered hydraulic dynamics in the rhizosphere. The experiments differ in their experimental set-up, the measured variables and in soil type, plant species and root age.

Appendix A is taken from the article Numerical simulation of coupled heat, liquid water and water vapor in soils for heat dissipation of underground electrical power cables by Kroener et al. (2014a) published in Applied Thermal Engineering. Soil thermal conductivity strongly depends on water content. Therefore it is important to couple water, vapor and heat flow to estimate the cables temperature in soil. We tested our numerical model on an down-scaled experiment from the literature and present numerical simulations under various conditions to show the relation between cable temperature, soil texture and atmospheric boundary conditions.

Appendix B is taken from the manuscript Estimation of thermal instabilities in soils for heat dissipation of underground electrical power cables by Kroener et al. (in preparation) submitted to Applied Thermal Engineering. Around the warm cable water evaporates, vapor diffuses away from the cable, condensates in the distant colder regions and liquid water flows back to the cable. This liquid return flow to the cable geometrically resembles the water flow towards a single root. Similar to root water uptake, this liquid return flow can not be sustained below a certain bulk soil water potential where the soil around the cable gets too dry and this water-vapor cycle breaks. Here we present an analytical estimation of the critical heat dissipation rate when the water-vapor cycle breaks as function of bulk soil water potential and the respective soil texture.

\footnotetext{
${ }^{3}$ E.K. was responsible for the modeling part and was partly involved in the experimental design of the study.
} 


\subsection{Material and Methods}

\section{Neutron radiography}

Neutron radiography is a non-invasive method that allows to monitor spatial distribution of water inside thin samples with high accuracy (Carminati et al., 2007). We used neutron radiography to monitor water content distribution in soils mixed with mucilage during drying and wetting. The neutron radiographs were taken at the NEUTRA and ICON imaging stations of the Paul Scherrer Institute, Switzerland (Kaestner et al., 2011).

The samples were placed in front of the neutron beam. A scintillator plate behind the sample converted the beam into visible light. The visible light was acquired by a digital camera and transformed into a digital image. The neutron attenuation coefficient of water is high compared to that of air and soil which makes neutron radiography an excellent technique to measure water content distribution non-destructively.

\section{Plant mucilage}

For Chapter 4 we used mucilage collected from maize roots - a method developed by Ahmed et al. (2015b). Since it is difficult to collect large amounts of mucilage from real plant roots, in some experimental studies (Chapter 2, 3, 5 and 6) we used mucilage collected from chia seeds (Salvia Hispanica L.) as an analogue for plant root exudates. The chemical composition and physical behavior of mucilage from maize roots and from chia seeds are similar: both are composed mainly of xylose, glucose and uronic acids. In both mucilages, the content of uronic acid is $\approx 25 \%$ (Lin et al., 1994, Carminati and Vetterlein, 2013). In fact, Lin et al. (1994) and Muñoz et al. (2012) have shown that when in contact with water mucilage of chia seeds formed a gel-like network with a remarkable capacity to adsorb water. Indeed, when immersed in water, within a few minutes mucilage appeared in form of a transparent capsule around the seeds (Fig. 1.2).

We used the sessile drop technique to measure the contact angle of mucilage dried at various concentrations on a flat glass surface. In the sessile drop technique a drop of water is placed on the treated surface, a high resolution camera takes a digital image and an image processing tool fits an ellipse to the imaged droplet to estimate the contact angle between liquid, solid and air. The measurements revealed that mucilage both form chia seeds as well as from maize roots turn hydrophobic at a concentration of around $0.03-0.06 \mathrm{mg} / \mathrm{cm}^{2}$ (Fig. 3.7 and Fig. 4.5. 


\section{Devices to measure soil hydraulic properties}

We used standard devices to measure soil hydraulic properties: the pressure plate apparatus was used to measure water retention curves. Here hollow cylinders of $6 \mathrm{~cm}$ in diameter and $6 \mathrm{~cm}$ in height were filled with the respective soil and were placed in the pressure plate apparatus. The water potential was reduced stepwise. In each step we waited until no outflow was visible for at least a few days. After each step we derived the water content from the weight of the sample.

We measured the saturated soil hydraulic conductivity using the constant head method as well as the falling head method. In the constant head method two different positive water potentials were applied on two sides of the sample. The soil hydraulic conductivity was obtained from the water flow through the sample, the difference in the applied water potentials and geometric extensions of the sample. In the falling head method a water column is connected to one side of the sample. The water column and as a result also the applied water potential continuously decrease as water flows through the sample. The hydraulic conductivity is obtained by fitting an exponential to the measured outflow rate.

We used a root pressure probe to measure the conductivity of the root-soil interface under various conditions. Here a suction cup is connected to a pressure transducer recording the pressure over time. By injecting or extracting water from soil the pressure inside the suction cup changes immediately. Now the relaxation of the pressure in the cup is related to the conductivity of the root-soil interface.
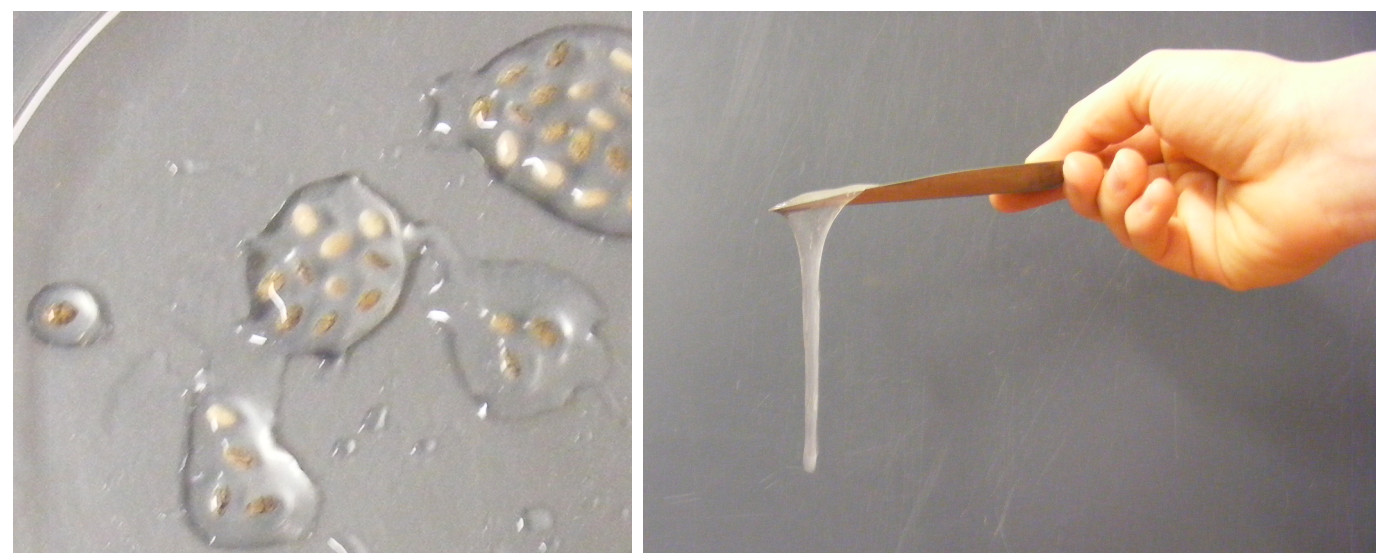

Figure 1.2: Left: mucilage appears in form of a transparent capsule around the seeds. Right: gel extracted from the seeds. 


\section{Numerical simulations}

For the numerical simulations of Chapter 5, 6 and 7 I adapted the Python-based code for infiltration of water into soil implemented by Bittelli et al. (2015) to our problem where water content and water potential are decoupled according to the concept of hydraulic nonequilibrium in the rhizosphere.

For Appendix B I modified this program to solve the coupled water, vapor and heat flow equation system describing the steady state problem of heat dissipation from an underground cable along the one-dimensional radial coordinate.

In Appendix A the coupled water, vapor and heat flow is solved numerically in a 2D crosssection of the soil surrounding the cable during a one-year period with measured weather data as upper boundary conditions. We used the finite element method to solve this problem and implemented the equations in PDELab (PDELab, 2014), a discretization module for partial differential equations. PDELab depends on the Distributed and Unified Numerics Environment (DUNE, 2014). Further informations can be found in Bastian et al. (2008b a); Blatt and Bastian (2007a). 


\title{
2 Roots at the Percolation Threshold
}

written by Eva Kröner, Mutez Ali Ahmed, Andrea Carminati, published in Physical Review E (Kroener et al., 2015)

\begin{abstract}
The rhizosphere is the layer of soil around the roots where complex and dynamic interactions between plants and soil affect the capacity of plants to take up water. The physical properties of the rhizosphere are affected by mucilage, a gel exuded by roots. Mucilage can absorb large volumes of water, but it becomes hydrophobic after drying. We used a percolation model to describe the rewetting of dry rhizosphere. We found that at a critical mucilage concentration the rhizosphere becomes impermeable. The critical mucilage concentration depended on the radius of the soil particle size. Capillary rise experiments with neutron radiography proved that for concentrations below the critical mucilage concentration water could easily cross the rhizosphere, while above the critical concentration water could no longer percolate through it. Our studies, together with former observations of water dynamics in the rhizosphere, suggest that the rhizosphere is near the percolation threshold, where small variations in mucilage concentration sensitively alter the soil hydraulic conductivity. Is mucilage exudation a plant mechanism to efficiently control the rhizosphere conductivity and the access to water?
\end{abstract}

\subsection{Introduction}

Worldwide $8.6 \cdot 10^{10} \mathrm{t}$ of water flow across the soil-plant-atmosphere-continuum every day (Dirmeyer et al., 2006). This water flow is necessary to sustain vegetation, carbon sequestration and food production. One of the least understood but very important interfaces in this water cycle is the rhizosphere, the soil region surrounding the roots and that is effected by them.

Since the early 60's the Gardner model (Gardner, 1960) has been applied to model root water uptake. In the Gardner model the Richards equation (Richards, 1931) is solved in radial coordinates and the root system is simplified as a single root. Since then, models have been developed that account for more complex 3D root architecture (Roose and Fowler, 2004; Doussan et al., 2006, Javaux et al., 2008). All of these models are based on the assumption of homogeneous properties around the roots. However, there is increasing evidence that the rhizosphere differs from the bulk soil in its physical, chemical and biological properties.

Root growth induces soil compaction in the vicinity of roots (Aravena et al., 2010, 2013). 

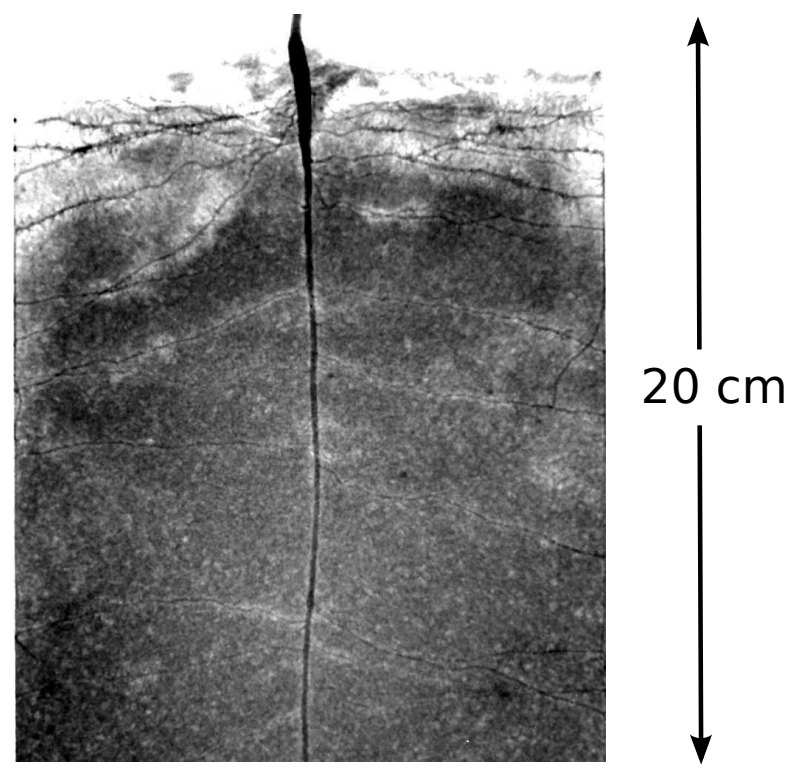

Figure 2.1: Water content distribution in the root zone of a young lupine after irrigation as imaged with neutron radiography. The grey values are proportional to the water content (dark=wet). The radiograph shows that the water content in the rhizosphere was markedly drier than in the adjacent bulk soil. The image is taken from the experiments described in Carminati (2013).

Variations in soil water content lead to expansion and shrinkage of roots and can produce air filled-gaps around roots under dry conditions (Carminati et al., 2013; North and Nobel, 1997). Furthermore, the rhizosphere is affected by mucilage, a polymeric gel exuded by roots. Mucilage is capable of absorbing large volumes of water, up to 1000 times its own dry weight (McCully and Boyer, 1997). Hydrated mucilage facilitates root water uptake from dry soil (Ahmed et al., 2014). However, when mucilage dries it turns hydrophobic and it makes the rhizosphere temporarily water repellent (Moradi et al., 2012) limiting root water uptake (Zarebanadkouki and Carminati, 2014).

Swelling and shrinking rates of mucilage within the soil pore space depend on mucilage elasticity and viscosity and they can be significantly slower than the change in water potential in the bulk soil near the root. The slow rewetting rate of the rhizosphere is illustrated in Fig. 2.1. which shows a neutron radiography of the root zone of a young lupine plant after irrigation subsequent to a drying period (Carminati, 2013). Carminati (2013) reported that during a drying period the rhizosphere of lupines had a higher water content than the adjacent bulk soil. In contrast, after irrigation the rhizosphere remained markedly drier than the bulk soil for approximately 1-2 days. However, the recovery in transpiration rate as well as root 


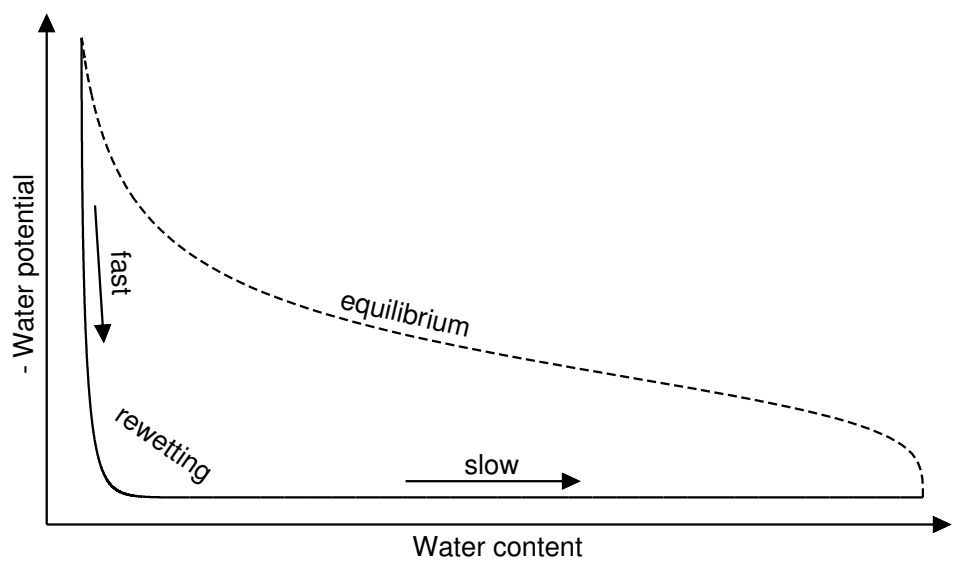

Figure 2.2: Non-equilibrium relation (Eq. 2.1) between rhizosphere water content and water potential in the rhizosphere during the rewetting phase (Kroener et al., 2014b). The water content recovers more slowly than the water potential, resulting in a deviation from the equilibrium curve.

swelling rates had faster kinetics, i.e. water was able to flow across the rhizosphere into the root without rewetting the rhizosphere significantly.

To describe these non-equilibrium dynamics Kroener et al. (2014b) proposed a dynamic relation between water content $\theta\left[\mathrm{cm}^{3} \mathrm{~cm}^{-3}\right]$ and water potential $\psi[\mathrm{hPa}]$ in the rhizosphere:

$$
\frac{\partial \theta}{\partial t}=R C_{\mathrm{bu}}(\psi) \frac{\mathrm{d} \psi}{\partial t}+(1-R) \frac{1}{\tau}\left[\psi-\psi^{e q}\left(\theta_{\mathrm{M}}\right)\right]
$$

where $R[-]$ and $\tau[\mathrm{s} \mathrm{hPa}]$ are parameters affecting the non-equilibrium dynamics, $\psi^{e q}\left(\theta_{\mathrm{M}}\right)$ [hPa] is the equilibrium water potential and $C_{\mathrm{bu}}(\psi)\left[\mathrm{hPa}^{-1}\right]$ is the bulk soil water holding capacity. The resulting dynamic relation is illustrated in Fig. 2.2.

When combined with the Richards equation (Richards, 1931) Eq. 2.1 is capable of reproducing the observed non-equilibrium dynamics in the bulk soil-rhizosphere-root-continuum. The model, however, increases the complexity of previous root water uptake models and adds further parameters that need to be fitted. Additionally, although Eq. 2.1 can effectively describe the observed hydraulic dynamics, it still needs to be physically justified at the pore scale.

The objective of this study was to understand the non-equilibrium dynamics using a percolation approach and to determine the concentrations where this non-equilibrium relation becomes relevant.

We applied a classic percolation model where a fraction of bonds is impermeable. An impermeable pore describes a pore covered with dry, hydrophobic mucilage. The percola- 
tion model predicts the fraction of disconnected pores above which the rhizosphere turns impermeable.

\subsection{Theory}

Percolation models are idealized representations of a medium at the microscopic scale used to derive macroscopic properties. Percolation has been applied to various disciplines: elasticity and formation of gels (Axelos and Kolb, 1990; Del Gado et al., 1998; De Gennes, 1976), metal-insulator transition (Meir, 1999, Gefen et al., 1986) and flow and transport in porous media (Ghanbarzadeh et al., 2014, Pike and Seager, 1974, Seager and Pike, 1974, Berkowitz and Ewing, 1998). When applied to hydraulic processes in soils, the pore space is usually represented as a regular lattice consisting of sites that are connected by bonds of simple shapes, i.e. cylindrical tubes, channels or angular prisms (Blunt, 2001; Thullner and Baveye, 2008; Ghanbarzadeh et al., 2014; Ebrahimi and Or, 2014). The importance of percolation for flow in porous media consists in relating large-scale flow phenomena at the Darcy scale to pore scale heterogeneities (

In this study we focus on water infiltration through the rhizosphere during the first minutes after irrigation. Consider an initially dry rhizosphere (Fig. 2.3 ). A pore covered with mucilage is initially water repellent (Fig. 2.3b). The small part of the gel in contact with water starts adsorbing water, wets and the contact angle relaxes. The wetting front advances and the gel continues swelling. The wetting of this pore is controlled by the advancing contact angle, the swelling rate of mucilage and the flow of water across mucilage. Since these processes happen at a time scale of several minutes to hours, we consider a mucilage covered pore as impermeable during the first minutes after irrigation. To incorporate this idea into a percolation model we set a random number of pores as covered with mucilage; these pores are assumed to be impermeable (Fig. 2.3k).

We simulated the percolation through a cubic lattice. We used the bond percolation model, in which the nodes between the sites can be either connected or disconnected. The simulations show a distinct dual behavior (Fig. 2.4 ): when $73 \%$ of the bonds are disconnected water can easily flow across the region, most nodes of the domain get filled with water, and the wetting front is rather uniform. At $74 \%$ water can still cross the region, but the wetting front becomes irregular and only a fraction of pores is rewetted. Above a percentage of $75 \%$ water can no longer percolate. This value is consistent with the more accurate value of $24.88126(5) \%$ of connected bonds determined by Lorenz and Ziff (1998) using extensive 
a)

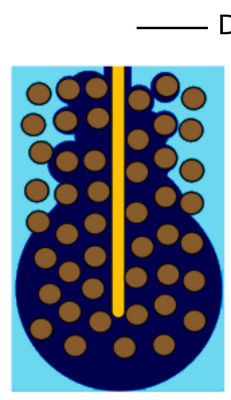

Drying $\longrightarrow \quad$ - Rewetting $\longrightarrow$

b)

1) dry mucilage is initially water

repellent 2) mucilage
wets and starts swelling

3) mucilage viscosity controls the conductivity

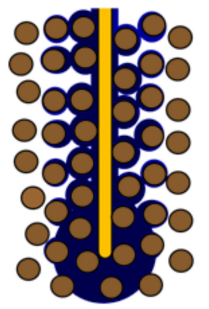

C)
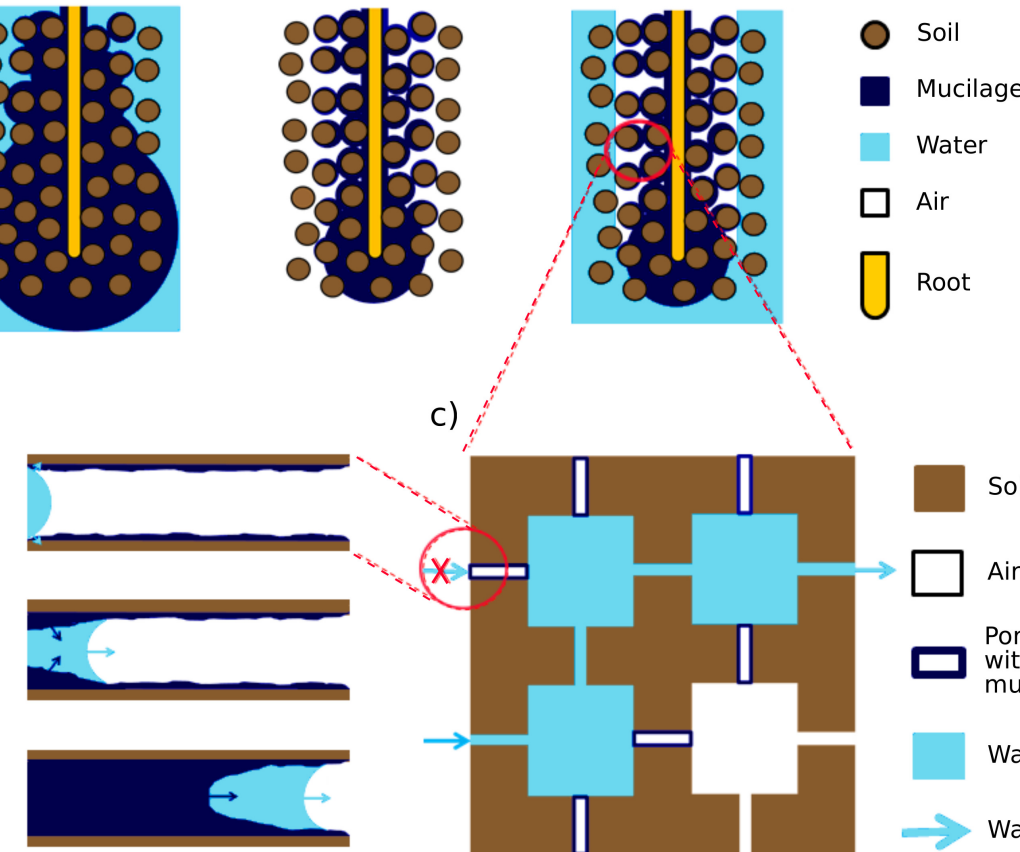
Air Pore covered wind mucilage

Water

Water flow

Figure 2.3: Conceptual model of rewetting of dry rhizosphere. a) Illustration of water content in the rhizosphere during drying and after rewetting; b) rewetting of a pore covered with dry mucilage; c) pore network model of bonds covered with mucilage (disconnected) and bonds not covered with mucilage (connected). 
Monte Carlo simulations. Close to the percolation threshold a small change in the number of disconnected nodes extremely alters the rhizosphere hydraulic behavior (Fig. $2.4 \mathrm{~b}, \mathrm{c}$ ).

Steenhuis et al. (2005) used a percolation model to explain water repellency in soils containing a fraction of hydrophobic particles. Experimentally, they found that $5 \%$ of hydrophobic particles can impede infiltration unless ponded. Our experiments differed in two ways: 1) all particles were uniformly treated; 2) the longitudinal dimension was shorter than the lateral ones - as in the rhizosphere, while in their theoretical considerations they followed the approach of Raikh and Ruzin (1990), who described the longitudinal conductivity of a narrow channel.

We did capillary rise experiments between pairs of glass plates to study the relation between mucilage concentration and contact angle. We used Chia seed mucilage (Salvia hispanica) since it is easy to extract and it has similar chemical composition as lupine and maize mucilage (mainly xylose, glucose and uronic acids, (Lin et al., 1994, Carminati and Vetterlein, 2013)). Additionally it has similar physical behavior: when in contact with water it becomes a gel (Lin et al., 1994; Muñoz et al., 2012) and it turns hydrophobic after being dried. Mucilage extraction from chia seeds is described in Kroener et al. (2014b); Ahmed et al. (2014). Our experiments showed that capillary rise in the angle between two glass plates was considerably reduced at a mucilage concentration of:

$$
c_{\mathrm{hy}}=6.0 \times 10^{-5} \mathrm{~g} \mathrm{~cm}^{-2}
$$

Capillary rise between glass plates that where covered with a mucilage concentration lower than $c_{\text {hy }}$ was finished within five minutes, while for plates covered with a higher concentration there was no capillary rise even after one day.

Once a threshold value of mucilage concentration per surface area was known, we calculated how much mucilage is needed to make the rhizosphere impermeable.

Assuming that the soil particles are spheres with radius $r$, the specific soil surface area $A$ $\left[\mathrm{cm}^{-1}\right]$ is:

$$
A=N 4 \pi r^{2}
$$

where $N\left[\mathrm{~cm}^{-3}\right]$ is the number of soil particles per unit of volume. $N$ is related to bulk soil density $\rho_{\mathrm{bu}}=1.6 \mathrm{~g} \mathrm{~cm}^{-3}$ and soil mineral density $\rho_{\mathrm{mi}}=2.6 \mathrm{~g} \mathrm{~cm}^{-3}$ according to:

$$
\rho_{\mathrm{bu}}=N \frac{4}{3} \pi r^{3} \rho_{\mathrm{mi}}
$$

Eqs. 2.3 and 2.4 give:

$$
A=\frac{3}{r} \frac{\rho_{\mathrm{bu}}}{\rho_{\mathrm{mi}}}
$$




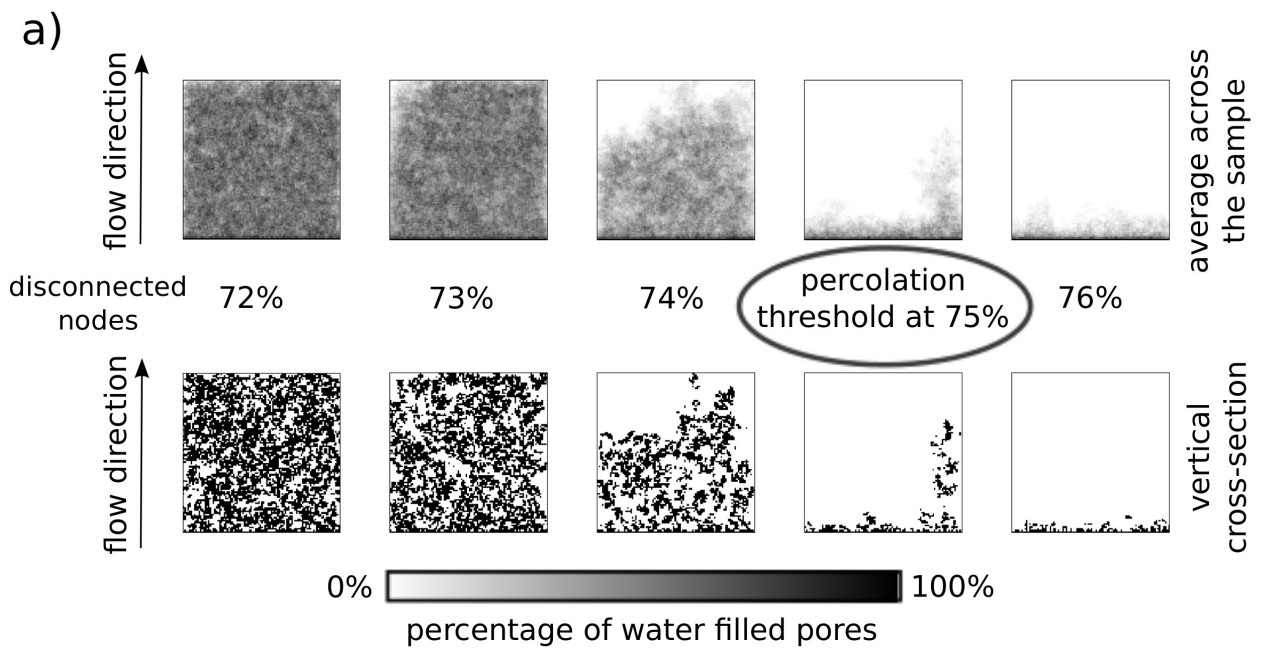

b)

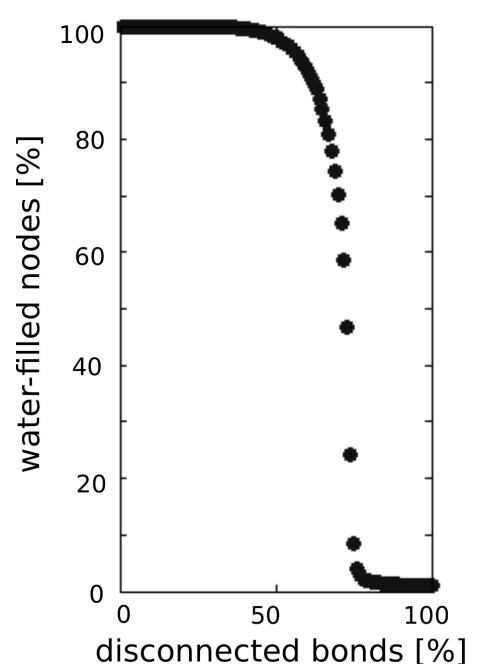

c)

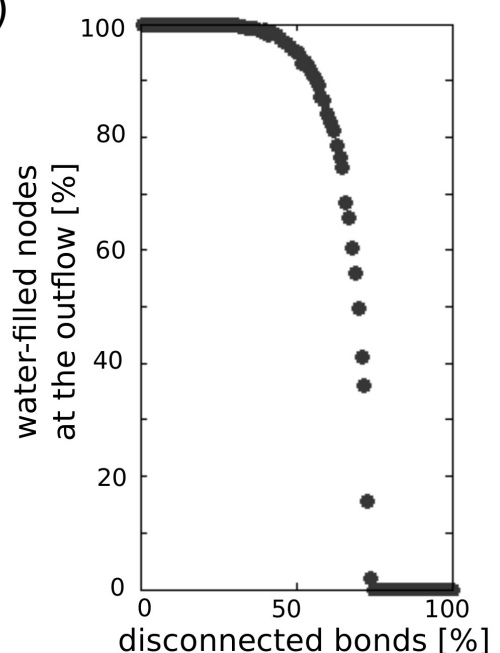

Figure 2.4: Bond percolation in a cubic lattice with varying percentage of disconnected pores.

a) Water filled pores averaged across the sample and for a horizontal cross section. b) Water filled nodes as a function of disconnected bonds. c) Water-filled nodes at the outflow as a function of disconnected bonds. Close to the percolation threshold a small change in the number of disconnected nodes strongly affects the hydraulic behavior. 
The cubic percolation model predicts that around $75 \%$ of disconnected nodes are sufficient to make the soil impermeable. This means that we expect the percolation threshold at a mucilage concentration of $c_{\mathrm{th}}=0.75 A c_{\mathrm{hy}}$. Combination with Eqs. 2.2 and 2.5 gives the mass of mucilage per unit volume of bulk soil required to make the soil impermeable:

$$
c_{\mathrm{th}}=\frac{1 \mathrm{~cm}}{r} 8.3 \times 10^{-5} \mathrm{~g} \mathrm{~cm}^{-3}
$$

Expressed as mass of dry mucilage per dry soil mass as a function of particle diameter $d$ it becomes:

$$
c_{\mathrm{th}}=\frac{1 \mathrm{~cm}}{d} 1.04 \times 10^{-4} \mathrm{~g} \mathrm{~g}^{-1}
$$

\subsection{Measurements}

We tested this theoretical relation by monitoring water infiltration through artificial rhizosphere layers of varying mucilage concentration and particle size. We mixed various amounts of wet mucilage with dry soil to obtain soil samples of the required mucilage concentration. The mucilage-soil mixture was dried for around 24 hours at $40^{\circ} \mathrm{C}$. To reduce accumulation of dry mucilage on the surface, we used very wide boxes, so that the height of the soil was less than $1 \mathrm{~mm}$.

We packed soil samples representing a simplified model of the root-rhizosphere-soil system: we used a layer of dry untreated soil as an analogue of the root, a layer of ca. $5 \mathrm{~mm}$ of treated soil as rhizosphere, and again a region of untreated soil representing the bulk soil.

Neutron radiography was used to monitor the water content distributions during capillary rise. Water has a high attenuation coefficient for neutrons, making of neutron radiography an optimal non-invasive method to quantitatively image water distribution in thin samples with

high accuracy (Carminati et al., 2007). The neutron radiographs were taken at the neutron imaging beam line NEUTRA (Pleinert and Lehmann, 1997) of the Paul Scherrer Institute, Switzerland.

The prepared dry samples were placed inside a box in front of the neutron beam. Then the box was filled with water until the water table was $2.5 \mathrm{~cm}$ below the layer representing the rhizosphere. The capillary rise in the samples was monitored with time-series neutron radiography. Fig. 2.5 shows the water content distribution in the samples five minutes after the start of capillary rise. A typical threshold behavior appeared: when the concentration of mucilage in the rhizosphere was below a certain value, then water could easily cross the treated layer. In contrast, when the concentration exceeded this value water could no longer cross this region - at least during the first hours after irrigation. Neutron radiography was 


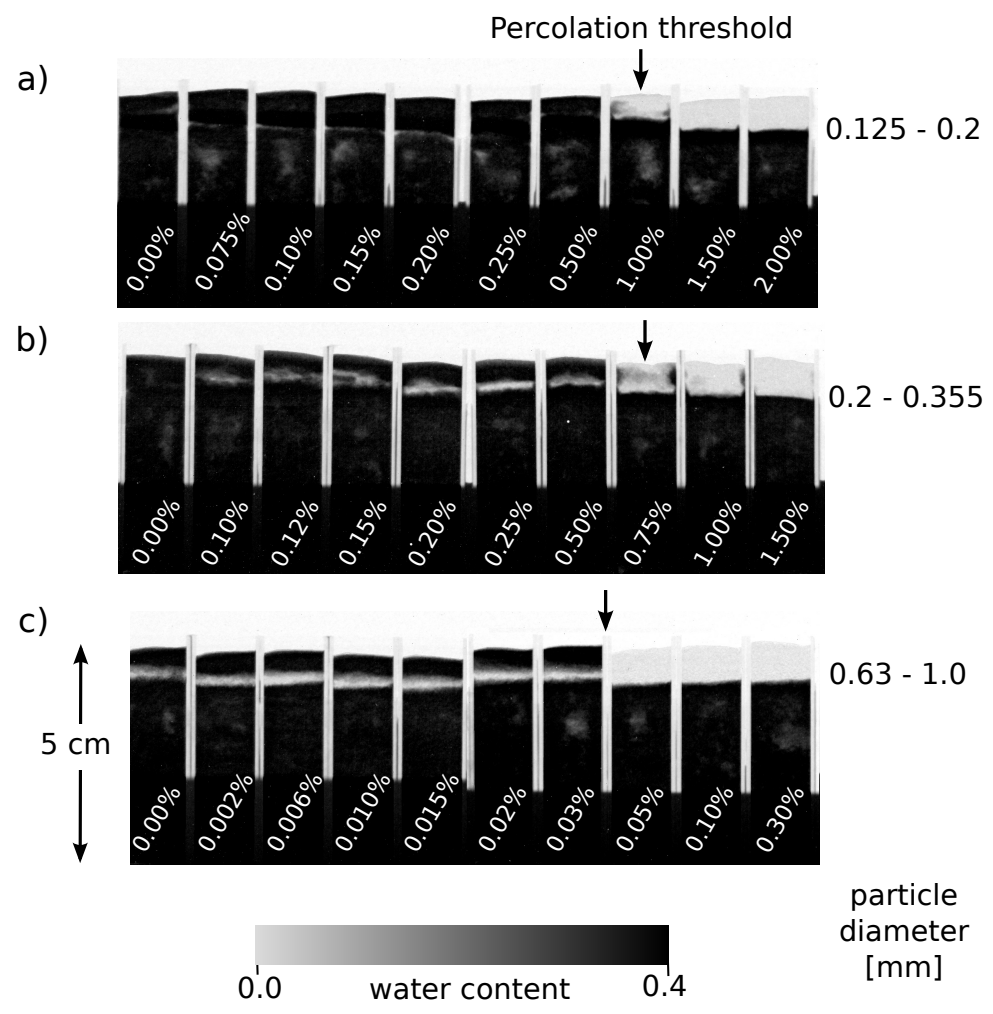

Figure 2.5: Capillary rise in soil columns with a small layer of soil mucilage mixture as an analogue of the rhizosphere. Among the soil columns particle size as well as mucilage concentration of the soil mucilage mixture varied: a) fine sand (diameter: $0.125-0.2 \mathrm{~mm}$ ), b) medium sand $(0.2-0.355 \mathrm{~mm}), \mathrm{c})$ coarse sand $(0.63-1.0 \mathrm{~mm})$. The gravimetric concentration (mass of dry mucilage per unit mass of dry soil) in the rhizosphere layer is given for each soil column. 


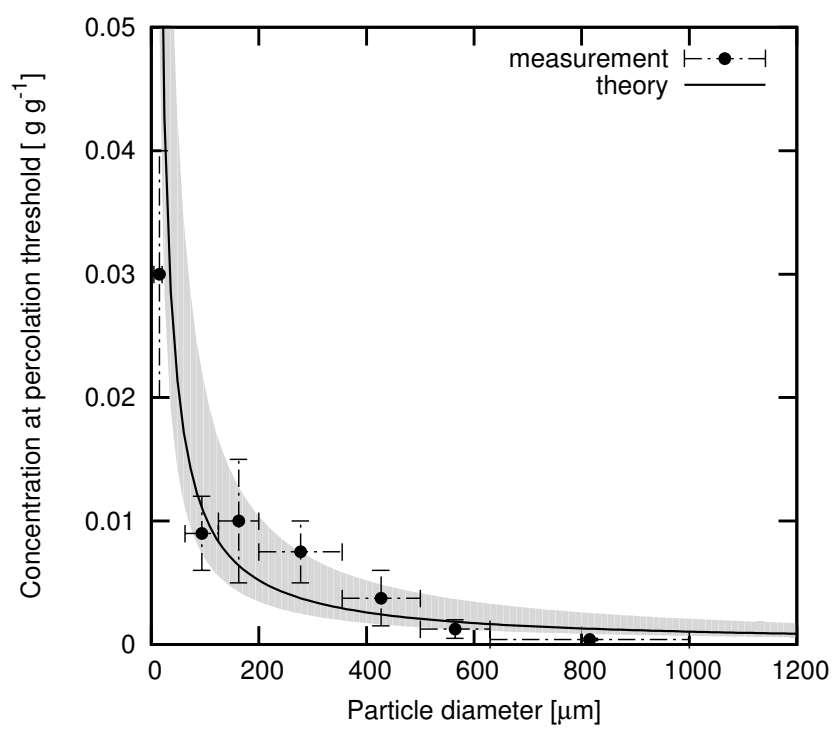

Figure 2.6: Relation between particle diameter and threshold concentration (Eqn. 2.7). The gray region is related to the error in the measurement of $c_{\mathrm{hy}}$.

performed with soils of three different particle sizes. Four additional particle sizes were tested in our laboratory. Note that to determine the mucilage concentration at the percolation threshold it is sufficient to control if the surface of the sample turns wet. In this way we determined further threshold concentrations for different particle sizes without using neutron radiography.

Fig. 2.6 shows the relation between mucilage concentration at the percolation threshold and particle size as predicted by Eq. 2.7 and as observed in our experiments. The experimental results fit well with the theoretical relation. For the coarse sand the experimental points are slightly below the range of theoretical values. This can be explained by the small offset in the water potential during the experiments. At $\psi=-2.5 \mathrm{~cm}$ the untreated, coarse rhizosphere layer (Fig. 2.5., sample on the left) was not fully saturated. Therefore less surface was needed to be covered by mucilage to prevent capillary rise, i.e. the mucilage concentration at percolation threshold is smaller than the one we expect from our theory developed for saturated soil conditions.

\subsection{Discussion}

Our pore network model and the capillary rise experiments showed a distinct dual behavior for the rewetting of dry rhizosphere. For mucilage concentrations lower than a certain threshold, water could uniformly and quickly cross the treated layer after rewetting of bulk soil. At 
slightly higher mucilage concentrations, the thin layer became almost impermeable. The mucilage concentration at the threshold strongly depended on particle size: the smaller was the particle size, the larger was the soil specific surface and more mucilage was needed to cover enough soil surface to induce water repellency. The percolation model could quantitatively reproduce well the threshold characteristics of the experiments.

Fig. 2.5p demonstrates that near the percolation threshold water could flow across the rhizosphere layer without rewetting it significantly. In equilibrium, however, mucilage should lead to a higher water content (Ahmed et al. 2014$)$. This apparent contradiction is explained by the slow rewetting of mucilage compared to initial percolation of water, which results in non-equilibrium dynamics in the rhizosphere (Kroener et al., 2014b).

Of course our percolation model contains several simplifications: a) Soils are not cubic lattices. More realistic networks, e.g. Voronoi networks, might provide a better prediction of the ratio of connected pores at the percolation threshold (Becker and Ziff, 2009). b) While percolation is scale independent, we expect our experimental results to depend on the thickness of the rhizosphere: in thicker soil layers mucilage is expected to start swelling during the percolation time, leading to pore clogging mechanisms that are not included in this model (Vandevivere and Baveye, 1992). Rhizosphere, however, is a very thin layer, so the percolation time should be much faster than the swelling. Therefore our model is justified to describe the initial phase of the rhizosphere rewetting. c) In our study the capillary pressure was almost zero. For lower capillary pressures, however, one can expect that a lower mucilage concentration is needed to make the rhizosphere impermeable.

Our experiments, together with former observations of water dynamics in the rhizosphere (Fig. 2.1), suggest that the rhizosphere is near the percolation threshold, where small variations in mucilage concentration sensitively alter the hydraulic behavior. It is tempting to conclude that mucilage exudation is an efficient mechanism to control the rhizosphere conductivity and the access to water. 


\title{
3 Water percolation through the root-soil interface 4
}

written by Pascal Benard, Eva Kröner, Peter Vontobel, Anders Kaestner, Andrea Carminati, published in Advances in Water Resources (Benard et al., 2015)

\begin{abstract}
Plant roots exude a significant fraction of the carbon assimilated via photosynthesis into the soil. The mucilaginous fraction of root exudates affects the hydraulic properties of the soil near the roots, the so called rhizosphere, in a remarkable and dynamic way. After drying, mucilage becomes hydrophobic and limits the rewetting of the rhizosphere. Here, we aim to find a quantitative relation between rhizosphere rewetting, particle size, soil matric potential and mucilage concentration. We used a pore-network model in which mucilage was randomly distributed in a cubic lattice. The general idea was that the mucilage concentration per solid soil surface increases the contact angle between the liquid and solid phases consequently limiting the rewetting of pores covered with dry mucilage. We used the Young-Laplace equation to calculate the mucilage concentration at which pores are not wettable for varying particle sizes and matric potentials. Then, we simulated the percolation of water across a cubic lattice. Our simulations predicted that above a critical mucilage concentration water could not flow through the porous medium. The critical mucilage concentration decreased with increasing particle size and decreasing matric potential. The model was compared with experiments of capillary rise in soils of different particle size and mucilage concentration. The experiments confirmed the percolation behaviour of the rhizosphere rewetting. Mucilage turned hydrophobic at concentrations above $0.1 \mathrm{mg} / \mathrm{cm}^{2}$. The critical mucilage concentration at matric potential of $-2.5 \mathrm{hPa}$ was ca. $1 \%[\mathrm{~g} / \mathrm{g}]$ for fine sand and $0.1 \%[\mathrm{~g} / \mathrm{g}]$ for coarse sand. Our conceptual model is a first step towards a better understanding of the water dynamics in the rhizosphere during rewetting and it can be used to predict in what soil textures rhizosphere water repellency becomes a critical issue for root water uptake.
\end{abstract}

\subsection{Introduction}

The rhizosphere is the layer of soil next to plant roots that is actively modified by root growth and exudation (Gregory, 2006, Hinsinger et al., 2009). This layer of soil has an extent of millimetres up to a maximum of a few centimetres and it has a profound impact on soil

\footnotetext{
${ }^{4}$ E.K. was responsible for the capilary rise experiments and contributed to the extension of the theoretical concept from the saturated case to the unsaturated case.
} 
hydrology. In fact, all the water that flows through the soil-plant-atmosphere continuum, which corresponds to $40 \%$ of the terrestrial precipitation (Bengough, 2012), flows across the rhizosphere. Sposito (2013) defined the flow of water through the rhizosphere as productive green water and suggested that rhizosphere processes are an essential element for sustainable and efficient use of soil-water resources.

The rhizosphere is where plants and soil meet and interact, and its physical, chemical and biological properties differ from those of the adjacent bulk soil (Hinsinger et al., 2009). In this study we focus on the physical properties of the rhizosphere and how they affect the water flow through the root-soil interface. Our starting-point are recent observations of water repellency in the rhizosphere (Carminati et al., 2010, Moradi et al., 2012). Carminati et al. (2010) found that the rewetting rate of the rhizosphere after a drying/wetting cycle was markedly slower than that of the bulk soil. They found that the rhizosphere of lupines subject to a drying cycle and then irrigated by capillary rise remained drier than the bulk soil for 1-2 days. Moradi et al. (2012) showed that the slow rewetting of the rhizosphere was caused by the high contact angle of dry rhizosphere. Carminati (2013) observed that rhizosphere hydrophobicity was more marked when the soil was let dry until the plants started to show wilting symptoms, which happened at volumetric water contents below 0.05 . However, water repellency in the rhizosphere was also visible when the samples were kept relatively wet (at volumetric water contents above 0.10-0.15). These observations suggest that rhizosphere water repellency is more evident in dry soils, but it also occurs in a broader range of water contents.

Carminati (2012) proposed that the water repellency of the rhizosphere resulted from mucilage exuded by roots. Mucilage is primarily exuded at the root tips and consists mainly of polysaccharides and a few percentages of phospholipids (Guinel and McCully, 1986; McCully and Boyer, 1997; Read et al., 2003). Guinel and McCully (1986) reported that mucilage of maize (Zea mays) had a remarkable ability to swell and adsorb water and found that at saturation the weight of wet mucilage is 1000 times higher than its dry weight. Read et al. (2003) measured similar mucilage water contents for fully hydrated maize mucilage. Impressed by the ability of mucilage to absorb water, McCully and Boyer (1997) tested whether mucilage can hold large volumes of water also when the water potential decreases. They found that most of the water stored in fully hydrated mucilage was actually lost at water potentials less negative than $-10 \mathrm{kPa}$. Nevertheless, Carminati (2012) argued that the remaining mucilage water content is sufficient to increase the soil moisture of a few 
percentages. Young (1995) came to a similar conclusion after measuring a higher water content in the rhizosphere of wheat compared to the adjacent bulk soil. Recently, Ahmed et al. (2014) and Kroener et al. (2014b) showed that mucilage exuded by chia seeds increases the water content of a sandy soil at any water potential if the system is in hydraulic equilibrium. Similar observations were made by Deng et al. (2015), who investigated the effect of mucilage exuded from the seed coating of Capsella bursa-pastoris L. Medik. on the water retention curve of a sandy clay loam. They found an increase in the saturated water retention due to the presence of seeds and/or mucilage. The increase in water content was visible for water potentials less negative than $-10 \mathrm{kPa}$.

Although these studies suggest a positive effect of mucilage on increasing the water content in the rhizosphere, other studies showed an apparently opposite behaviour. Read and Gregory (1997) observed that mucilage surface tension was smaller than the one of water. Read et al. (2003) explained this reduction in surface tension as the effect of phospholipids. The reduction of surface tension is likely to decrease the capillary forces and to reduce the soil water content at negative water potential, as shown for an analogue of mucilage phospholipids (lecithin) mixed with a sandy loam (Read et al., 2003). The importance of the surface tension of mucilage is currently poorly understood, but it is likely to play an important role on rhizosphere hydrology, in particular in shaping the initial penetration of mucilage through the soil pores.

Upon drying, the phospholipids present in mucilage are expected to induce some degree of water repellency. In fact, Czarnes et al. (2000) observed that soils mixed with polygalacturonic acid (one of the main components of mucilage) became water repellent. Hallett et al. (2003) investigated the impact of four plant species on the hydraulic properties of rhizosphere soil and observed different magnitudes of water repellency among the samples. Furthermore, they suggested the idea that the rewetting rate of mucilage was reduced by mucilage swelling and consequent pore clogging. Moradi et al. (2012) measured contact angles higher than $90^{\circ}$ in the rhizosphere of lupine roots. Carminati et al. (2010) used neutron radiography, to image the water content distribution around the roots of lupines in a sandy soil. They found that during drying the rhizosphere was wetter than the bulk soil. On the contrary, after irrigation the rhizosphere remained dry and it rewetted in a few days. Carminati (2012) suggested that these two apparently contrasting behaviours are not in contradiction, but they are rather the result of the dynamic and time-dependent effect of mucilage on the hydraulic properties of porous media. At equilibrium conditions mucilage is capable of holding large 


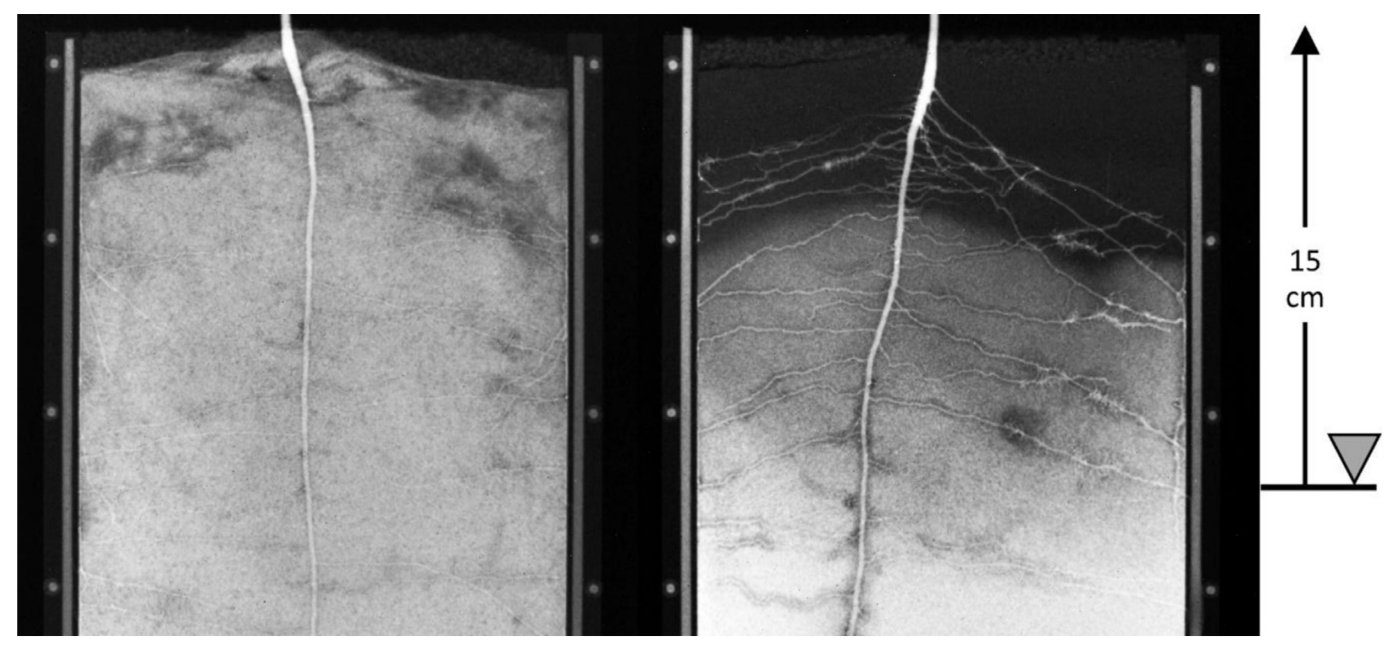

Figure 3.1: Neutron radiographs of water distribution around the roots of lupines after irrigation. Left: lupine in a sandy soil; right: lupine in a coarse quartz sand. The samples were rewetted by capillary rise, with the water table at a depth of $15 \mathrm{~cm}$ from the soil surface. The grey values are proportional to the water content (dark=dry, bright=wet). Dry zones are visible around the roots, in particular in the coarse quartz sand (right).

volumes of water and it increases the soil water content at any water potentials Kroener et al. 2014b). After drying mucilage turns hydrophobic and delays the rhizosphere rewetting for a period of hours up to a few days (Carminati, 2012), resulting in time-dependent and hysteretic hydraulic properties of the rhizosphere (Kroener et al., 2014b).

In the present study we investigate the water dynamics in the rhizosphere during the initial stage of the rewetting phase after the soil was let dry. Our objective was to quantitatively relate the rewetting kinetics of the rhizosphere to mucilage concentration, soil particle size and soil water potential.

To better motivate our study we refer to Fig. 3.1. which shows the water content distribution around the roots of lupines a few minutes after rewetting subsequent to a severe drying. The grey values are proportional to the water content (dark=dry, bright=wet). The images were obtained using neutron radiography. The details of the experiments are described in Carminati (2013). The samples were $30 \mathrm{~cm}$ high and were irrigated by capillary rise, with the water table being placed at $15 \mathrm{~cm}$ below the soil surface. The lupine plant on the left side was grown in a sandy soil composed of coarse sand (6.59\%), medium sand (49.9\%), fine sand $(31.5 \%)$, coarse silt (3.35\%), medium silt (1.8\%), fine silt (1.81\%) and clay (5.05\%). The lupine on the right side was grown in quartz sand with a particle diameter of 0.2 to $0.63 \mathrm{~mm}$. 
The radiographs show that in both samples the water content in the direct vicinity of roots was reduced, but the reduction was much more marked and evident in the coarser quartz sand (sample on the right side). Note that the reduction in water content near the roots was caused by the altered contact angle of the rhizosphere and could not be trivially explained as the effect of water depletion due to root uptake. Indeed, the soil water content in the two samples was high enough to allow a fast redistribution of water in the soil. The water that roots took up from the rhizosphere was very quickly replaced by water flowing from the bulk soil adjacent to the rhizosphere. Moderate water depletion around the roots could only been expected in drier soil conditions and not at such high water contents.

Our objective was to develop a conceptual model to explain the differences between the rhizosphere of the two samples shown in Fig. 3.1. We aimed to find the relation between rhizosphere rewetting, soil particle size, mucilage exudation and soil matric potential. We intended to answer the following questions. At what mucilage concentrations does a soil with a given particle size become water repellent? What matric potential is needed to rewet a rhizosphere with a given mucilage concentration and particle size?

To answer these questions we: (1) performed capillary rise experiments with soils of varying particle size and mucilage concentration; (2) developed a simple model of water percolation across the rhizosphere.

The concept behind the model is that the distribution of mucilage at the pore-scale is heterogeneous. This microscopic heterogeneity of mucilage deposition on the pore surface results in a distribution of contact angles in the porous medium. The mucilage concentration on the pore surface affects the contact angle between water and the solid surface and determines the wettability of a pore at a given water potential. If the fraction of wettable pores is high enough to form a connected network spanning throughout the sample water could flow across the porous medium; which is a typical percolation problem.

Percolation theory has been used to predict macroscopic transport properties of porous media by simulating relevant processes at the microscopic scale Berkowitz and Balberg, 1993, 1992; Berkowitz and Ewing, 1998; Hunt, 2004). Steenhuis et al. (2005) used the percolation approach to explain the experimental observation that a few hydrophobic grains can make a soil water repellent. We followed a similar percolation approach to predict how the microscopic distribution of mucilage at the pore-scale affects the macroscopic rewetting of the rhizosphere. To test our conceptual model, we used neutron radiography to monitor the water flow through soils and quartz sands of varying particle size mixed with different 


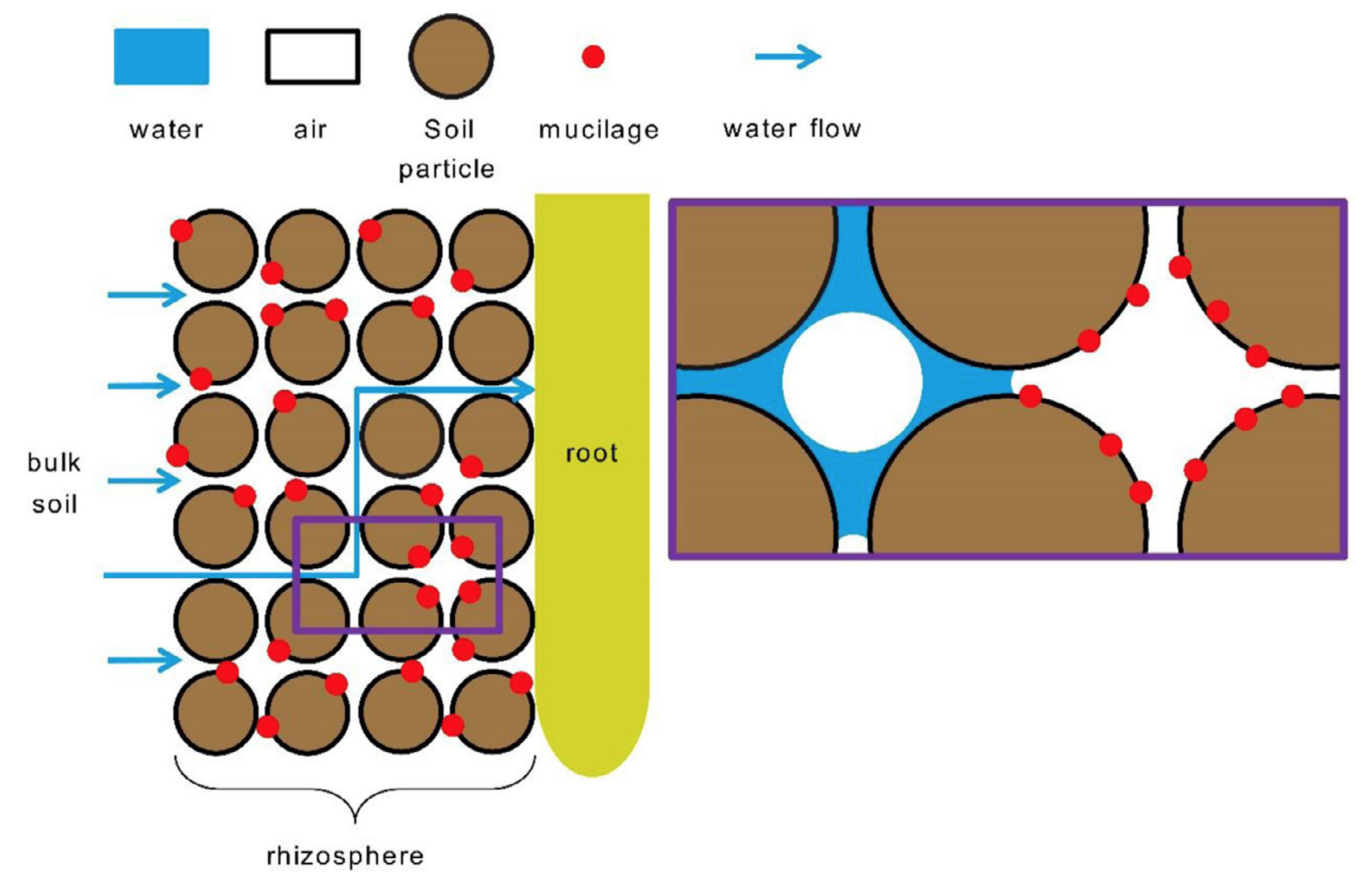

Figure 3.2: Illustration of the effect of mucilage on water flow in the rhizosphere. After drying mucilage becomes hydrophobic and impedes the water flow in a fraction of the pores.

quantities of mucilage. Additionally, we measured the contact angle of glass slides covered with varying quantities of mucilage.

\subsection{Conceptual Model}

Mucilage is exuded from the root tips and it diffuses into the soil until it binds to the surface of soil particles. It is expected that during the first drying phase mucilage keeps the rhizosphere wet. As roots take up water and the soil dries out, mucilage dehydrates, turns hydrophobic and causes high water repellency in the rhizosphere. At this point, even when the bulk soil is rewetted the rhizosphere remains dry. Afterwards, mucilage slowly rehydrates, the contact angle relaxes and the rhizosphere rewets. The rewetting of the rhizosphere may vary from a period of some hours up to a few days (Carminati, 2012). In this study we focus on the initial phase of the rewetting process and we do not consider mucilage swelling and the rewetting of the rhizosphere. This is justified by the different time-scales of the two processes: the initial water flow through the rhizosphere has a time scale of seconds to a few minutes, the rehydration of mucilage has a time scale of hours.

An illustration of the model is shown in Fig. 3.2. Dry mucilage is randomly distributed on the surface of the soil particles. The quantity of mucilage on the pore surface affects 
the contact angle between water and the solid particle. We assume that the contact angle depends on the mucilage concentration on the pore surface $C_{S}\left[\mathrm{~g} / \mathrm{cm}^{2}\right]$ - i.e. $C_{S}=\alpha\left(C_{S}\right)$. We expect that the contact angle increases with increasing mucilage concentration per soil surface $C_{S}$. It follows that the wettability of a pore depends on both, the specific pore surface and the quantity of mucilage in the pore. We also assumed that mucilage distribution and soil particle position is constant over time.

The wettability of a pore is estimated according to the Young-Laplace equation. A pore is rewetted when the following condition is satisfied:

$$
h>\frac{-2 \gamma \cos (\alpha)}{r \rho_{w} g}
$$

where $h$ is the matric potential (or capillary pressure) expressed in centimetre heads [cm] (note that in unsaturated conditions $\mathrm{h}$ is negative), $\gamma$ is the surface tension of water $\left[\mathrm{J} \mathrm{m}^{-2}\right.$ ], is the contact angle, $\rho_{w}$ is the density of water $\left[\mathrm{g} \mathrm{cm}^{-3}\right], \mathrm{r}$ is the pore radius $[\mathrm{cm}]$ and $\mathrm{g}$ is gravity $\left[\mathrm{m} \mathrm{s}^{-2}\right]$. By using the Young-Laplace equation to estimate the wettability of a pore, we assume that pores are cylindrical and we ignore corner and film flows in more realistic porous media. Recent and more advanced pore-network models can be found in Blunt (2001), Valvatne and Blunt (2004), Joekar-Niasar and Hassanizadeh (2012). Here we used a very simple representation of the porous medium because of the lack of experimental information on the microscopic distribution of mucilage in the pores. Since this information is missing, we decided to start with a simple model.

Similarly, we simplified the pore-network as a cubic lattice, with each cube representing a single pore. Assuming that the soil was a cubic packing of spheres, we obtained that the radius of the embedded pore was 0.73 times the radius of the soil particles.

Then we randomly distributed the mucilage in the cubic packing. A random, normally distributed quantity of mucilage per pore was generated. The distribution was shifted towards the positive direction by summing to all values the largest absolute value of the distribution. Then the values were scaled to obtain the desired quantity of mucilage in the packing. A distribution of mucilage per pore is shown in Fig. 3.3

Based on the mucilage distribution and the particle radius, we calculated the surface mucilage concentration $C_{S}$ in each pore. Given the relation between contact angle $\alpha$ and $C_{S}$, we used Eq. (3.1) to predict if a pore of a given radius $r$, at a matric potential $h$ is rewettable or not. According to Stauffer (1985), the percolation threshold for a cubic lattice is $31.17 \%$. In other words, at least $31.17 \%$ of the sites must be conductive to allow the water flow. The line in Fig. 3.3 shows the distribution of mucilage at the percolation threshold: the pores on 


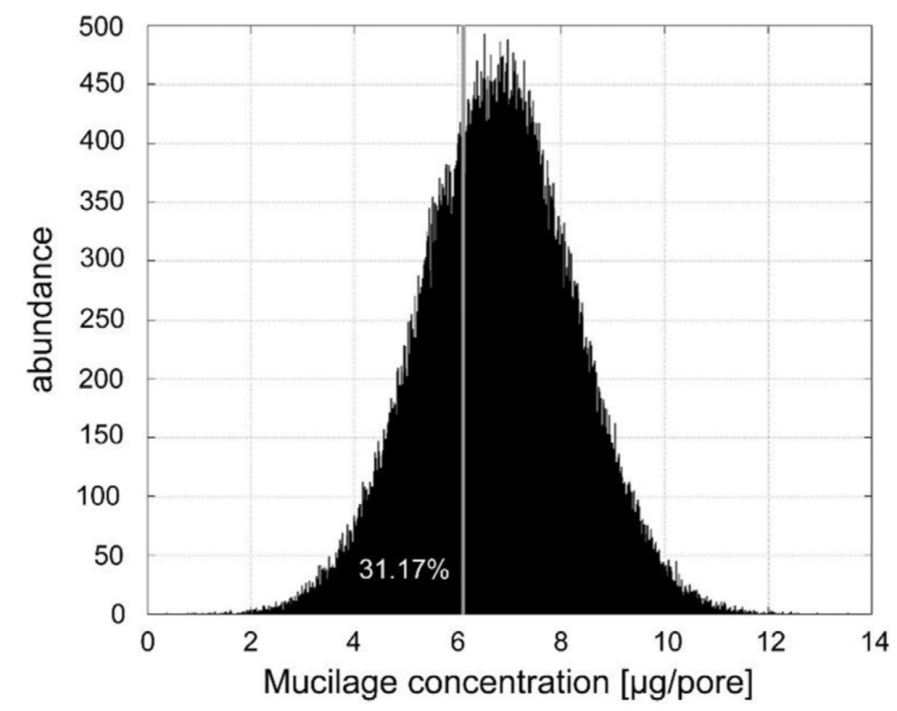

Figure 3.3: Example of mucilage distribution in the pore-network model. According to the percolation theory, at least $31.17 \%$ of the pores must be conductive to enable the water flow from one side of the system to the opposite side. Eq. 3.1) allows to calculate if a pore of given radius and mucilage concentration $C_{S}$ is wettable at a given matric potential $h$.

the left side of the line satisfy Eq. (3.1) and are wettable, while the pores on the right side are not wettable.

After the distribution of mucilage, each pore was checked for possible invasion. The process of invasion was started from one face (starting face) of the cubic lattice to its opposite side. A pore was invaded when Eq. (3.1) was satisfied and the pore was connected to a wetted pore (for each pore we considered the 6 neighbours). An illustration of the process close to the percolation threshold is shown in Fig. 3.4 .

In Fig. 3.4 we showed the invasion of water from the left side for varying mucilage concentration, $C[\mathrm{~g} / \mathrm{g}]$, defined as the gram of mucilage per gram of dry soil. At a critical mucilage concentration, $C_{t h}$, the infiltration front became irregular and formed fingers, as often observed in hydrophobic soils (Bauters et al. 2000). Slightly above and below $C_{t h}$ the final water content differed largely.

Objective of the model was to predict $C_{t h}$ as a function of particle size and soil matric potential. Note that as the soils used in the experiments had a narrow pore size distribution, in the model we assumed a uniform pore size. Of course, our model is a clear simplification of water flow in porous media. Beside ignoring film flow and snap off mechanisms, we also simplified the pore network as a cubic lattice, which has a coordination number equal to 6 , 


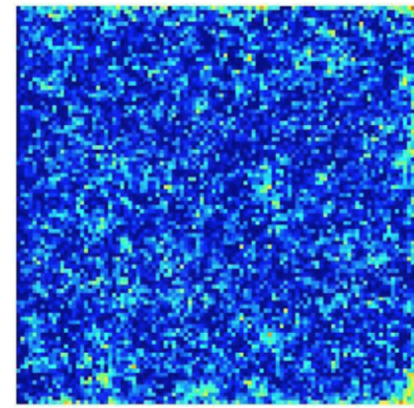

$0.0114 \mathrm{~g} / \mathrm{g}$

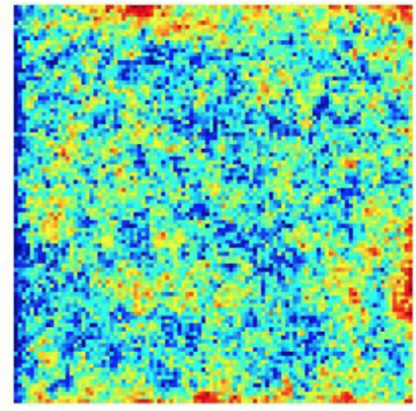

$0.0116 \mathrm{~g} / \mathrm{g}$

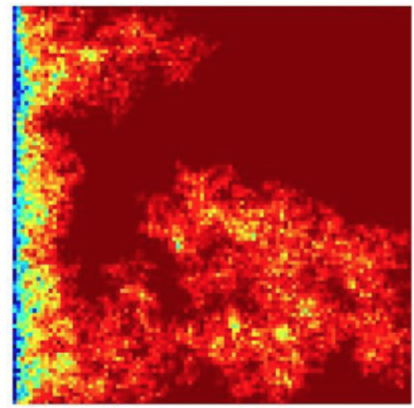

$0.0118 \mathrm{~g} / \mathrm{g}$

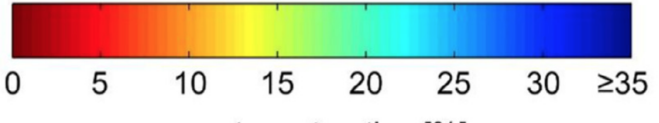

water saturation [\%]

Figure 3.4: Simulation of water percolation through a soil with varying mucilage concentrations. The simulations show the invasion of water from the left to the right in a soil with pore radius $r=0.1625 \mathrm{~mm}$ at matric potential $h=-2.5 \mathrm{~cm}$. Near $C_{t h}$ small changes in mucilage concentrations resulted in a large change in water saturation.

while the coordination number of real porous media is variable (Valvatne and Blunt, 2004). We intentionally kept the model as simple as possible, so that we could investigate the effect of a few parameters, soil particle size and mucilage concentration on the water repellency in the rhizosphere.

\subsection{Material and Methods}

To reproduce an analogue of the rhizosphere, we mixed soils of different particle size with a varying quantity of mucilage, obtaining a range of mucilage concentrations $C$ (dry mass of mucilage per dry mass of soil). We extracted mucilage from the seeds of chia (Salvia hispanica L.). We chose mucilage from chia seeds since it can easily be extracted and it has a similar chemical composition to lupine and maize mucilage (mainly xylose, glucose and uronic acids) (Lin et al., 1994). Additionally, it also has similar physical properties - i.e. it becomes a gel after immersion in water and it turns hydrophobic after drying Ahmed et al., 2014; Kroener et al. 2014b). Mucilage was extracted according to the procedure described in Ahmed et al. (2014) and Kroener et al. (2014b).

By mixing various amounts of wet mucilage with dry soil, we obtained samples with varying $C$. The gravimetric water content of the mixture was around $25 \%$ to ensure a homogeneous mucilage-soil mixture. Experiments were conducted with a silty soil (particle diameter $d=0-0.02 \mathrm{~mm})$ and quartz sands of different particle diameters $(d=0.063-0.125$ 
Table 3.1: Soil particle diameters and estimated average surface area $A$ assuming a cubic packing of spheres.

\begin{tabular}{ll}
\hline Soil particle diameter $[\mathrm{mm}]$ & Surface area A $\left[\mathrm{cm}^{2} \mathrm{~g}^{-1}\right]$ \\
\hline $0-0.02$ & 2308 \\
$0.063-0.125$ & 245 \\
$0.125-0.2$ & 142 \\
$0.2-0.355$ & 83 \\
$0.355-0.5$ & 54 \\
$0.5-0.63$ & 41 \\
$0.63-0.1$ & 28 \\
\hline
\end{tabular}

$\mathrm{mm}, d=0.125-0.2 \mathrm{~mm}, d=0.2-0.355 \mathrm{~mm}, d=0.355-0.5 \mathrm{~mm}, d=0.5-0.63 \mathrm{~mm}$, and $d=0.63-1 \mathrm{~mm}$ ). Assuming that the soil particles had spherical shape and uniform particle size distribution, we estimated that the surface area per gram of soil is $A=\frac{6}{d} \frac{1}{\rho_{m i}}\left[\mathrm{~cm}^{2} / \mathrm{g}\right]$, where $d$ is the particle diameter and $\rho_{m i}$ is the mineral density $\left(2.6 \mathrm{~g} / \mathrm{cm}^{3}\right)$. The specific surface area $A$ of the different soils is given in Table 3.1 .

The mucilage-soil mixtures were dried for about $24 \mathrm{~h}$ at $40^{\circ} \mathrm{C}$. To avoid accumulation of dry mucilage on the surface, the mixture was spread on a large plate with the height of the mixture being smaller than $1 \mathrm{~mm}$. The mucilage-sand composition formed a crust which was then poured through a sieve to separate individual grains.

The experimental set-up consisted of thin rectangular containers of various heights and base of $1.3 \mathrm{~cm} \times 1.3 \mathrm{~cm}$. The containers were filled with three soil layers. The lower part of each sample was filled with untreated soil. The second layer was filled with the different soilmucilage mixtures. This layer had a thickness of $0.5 \mathrm{~cm}$ and represented the rhizosphere. The upper layer consisted of untreated sand. The samples were initially dry. Then the samples were immersed in water until the water table reached a given height below the second layer (the rhizosphere). The tested distances between the water table and the rhizosphere were: $2.5 \mathrm{~cm}, 6.5 \mathrm{~cm}$ and $12 \mathrm{~cm}$ (Fig. 3.5).

The water content in the samples was monitored in real time using neutron radiography. Neutron radiography is an optimal non-invasive method to measure the spatial distribution of water in thin samples with high accuracy (Carminati et al., 2007). Neutron radiographs were performed at the NEUTRA and ICON imaging stations of the Paul Scherrer Institute, Switzerland (Kaestner et al., 2011). 


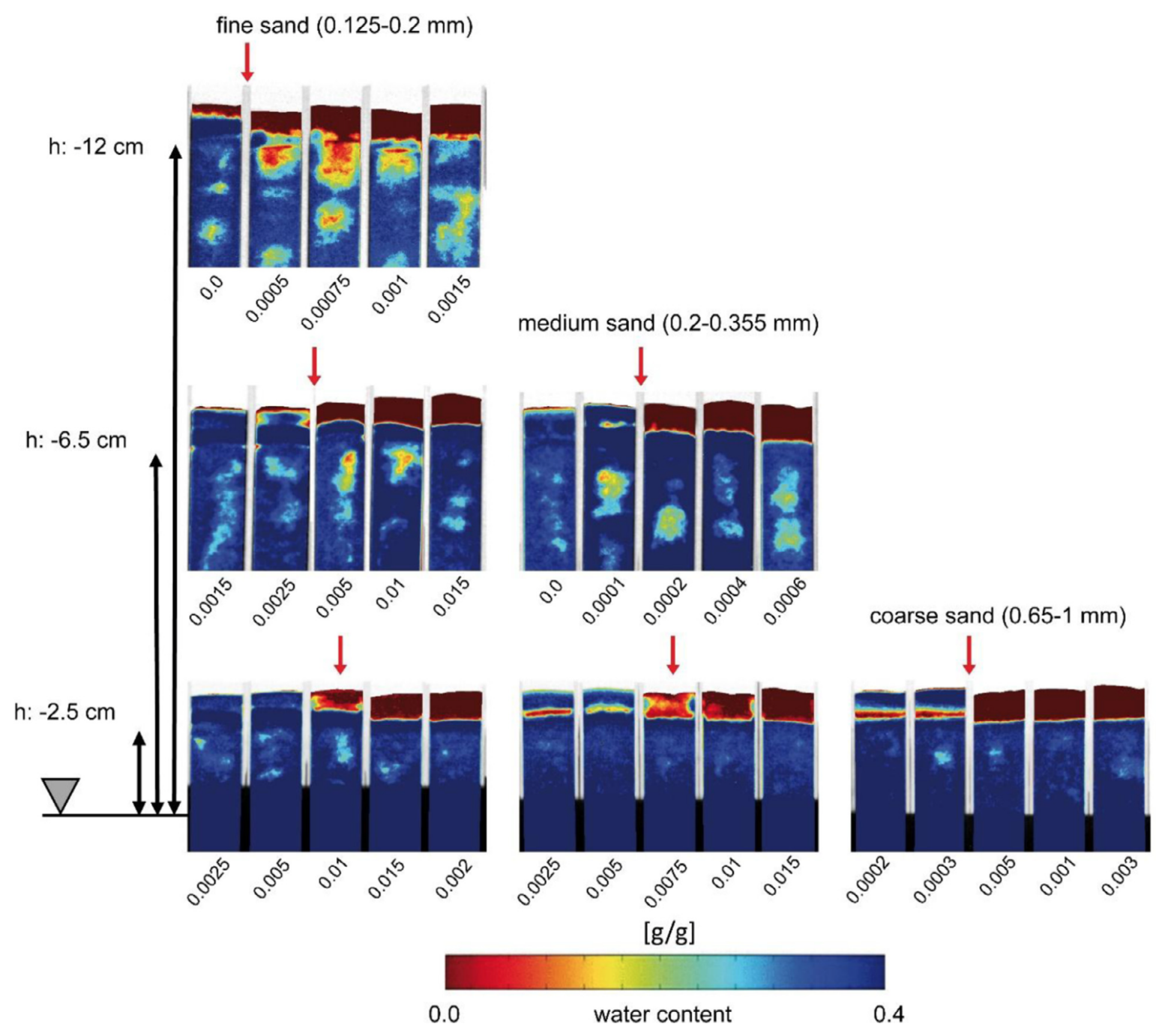

Figure 3.5: Neutron radiographs of the capillary rise experiments in fine sand (particle diameter $0.125-0.2 \mathrm{~mm})$, medium sand $(0.2-0.355 \mathrm{~mm})$ and coarse sand $(0.65-1 \mathrm{~mm})$ at varying matric potentials, $\mathrm{h}=-2.5 \mathrm{~cm}, \mathrm{~h}=-6.5 \mathrm{~cm}$ and $\mathrm{h}=-12 \mathrm{~cm}$. Each sample was prepared with a specific amount of mucilage. The threshold mucilage concentrations $C_{t h}$ are indicated with an arrow. 
Objective of the neutron radiography experiments were: (1) to investigate whether there was a critical mucilage concentration $C_{t h}$ [gram of dry mucilage per gram of dry soil] above which water could not percolate through the rhizosphere layer; (2) to experimentally estimate $C_{t h}$ for varying soil-mucilage mixtures and matric potential.

Then we tested if our percolation model was able to reproduce the observed $C_{t h}$. To fit the values of $C_{t h}$, we let vary the relation between the contact angle and the surface mucilage concentration $C_{S}$. Note that is the microscopic contact angle at the pore-scale.

An initial estimation of the microscopic contact angle as a function of mucilage concentration, $\alpha\left(C_{S}\right)$, was obtained from capillary rise experiments between two glass slides. The glass slides were prepared with specific amounts of mucilage. Diluted wet mucilage was uniformly spread on the glass slides and it was let dry at $40^{\circ} \mathrm{C}$ for $24 \mathrm{~h}$. The slides were then taped together on one corner, while a clip was placed between them in the other corner. In this way a narrow angle formed between the slides. The slides were then placed in dyed water and the capillary rise was monitored. The experiments were conducted several times, with values of $C_{S}$ ranging from 0 to $5.46 \mathrm{e}-4 \mathrm{~g} / \mathrm{cm}^{2}$. Values between these concentrations were chosen with $5.46 \mathrm{e}-4 \mathrm{~g} / \mathrm{cm}^{2}$ multiplied by $1 / 3,1 / 9,1 / 16,1 / 27$ and 0 .

The capillary rise experiments between the glass plates gave an initial range of values for the relation between $\alpha$ and $C_{S}$. Within this range, we determined the curve $\alpha\left(C_{S}\right)$ that gave the best fit between the model and the neutron radiography experiments.

To validate the model, we additionally measured the curve $\alpha\left(C_{S}\right)$ using the sessile drop method (SDM). Slides with specific amounts of dry mucilage were prepared as described above. Then a water droplet was placed with a needle on the slides and the initial contact angle was measured optically at $25^{\circ} \mathrm{C}$ (Drop Shape Analyser DSA25S; KRUSS GmbH). We measured the contact angle for samples with a surface concentration $C_{S}\left[\mathrm{~g} / \mathrm{cm}^{2}\right]$ ranging from 0.00015625 to $0.32 \mathrm{mg} / \mathrm{cm}^{2}$. Measurements were repeated at least 10 times for each $C_{S}$. These measurements provide the relation between contact angle and surface mucilage concentrations of $C_{S}$.

\subsection{Results and Discussion}

The neutron radiographs showed that at a critical mucilage concentration $C_{t h}$ there was a sudden transition in the wettability of the soil-mucilage layer. At mucilage concentrations below $C_{t h}$ water could rapidly flow through the layer, while at concentrations above $C_{t h}$ the treated layer impeded the water flow for more than one hour. Exemplary radiographs of 
the experiments are shown in Fig. 3.5. The images show the water content in the samples 5 minutes after irrigation from the bottom. The water content was derived by referencing the actual radiographs to the radiograph of the dry samples. The detailed image analysis is described elsewhere in details (Carminati et al., 2007; Hassanein et al., 2005). The colours in the images show the water contents. The threshold mucilage concentration $C_{t h}$ at the transition between the conductive and the non-conductive state depended on particle size and matric potential. $C_{t h}$ decreased with increasing particle size and with decreasing matric potential. For a matric potential of $-2.5 \mathrm{~cm}$ and particle diameter of $0.125-0.2 \mathrm{~mm} C_{t h}$ was around $1 \%[\mathrm{~g} / \mathrm{g}]$. For particle diameters of $0.2-0.355 \mathrm{~mm}$ at the same matric potential of -2.5 $\mathrm{cm} C_{t h}$ was $0.75 \%$. At a matric potential of $-6.5 \mathrm{~cm} C_{t h}$ was around $0.375 \%$ for $0.125-0.2$ $\mathrm{mm}$ particle diameter and $0.015 \%$ for $0.2-0.355 \mathrm{~mm}$ particle diameter.

The simulation of the capillary rise experiments using the pore- network model showed a good agreement with the neutron radiographs. The threshold concentration $C_{t h}$ as derived from the neutron radiography experiments and as predicted by the pore-network model are shown in Fig. 3.6. The effect of particle size and matric potential on $C_{t h}$ was well described by the model. Soils with large particle diameters have a small specific surface - i.e. $A$ is proportional to $1 / d$. This results in high concentration of mucilage per surface area $C_{S}$ and high contact angles. Additionally large particle diameters decrease the capillary forces (Eq. (3.1)), resulting in a lower critical mucilage concentration $C_{t h}$. Similarly, according to Eq. (3.1) a decrease in matric potential results in a decrease in $C_{t h}$; in other words, at more negative water potentials, less mucilage is needed to impede the water flow.

The relation between $\alpha$ and $C_{S}$ is plotted in Fig. 3.7. The initial estimation of the relation between $\alpha$ and $C_{S}$ was derived from the capillary rise experiments between two glass plates (region between the two lines). The experiments showed that for $2.02 \mathrm{e}-5 \mathrm{~g} / \mathrm{cm}^{2}<C_{S}<1.82 \mathrm{e}-$ $4 \mathrm{~g} / \mathrm{cm}^{2}$ there was no capillary rise between the slides, indicating that $\alpha \geq 90^{\circ}$. At the other extreme, we found that for $C_{S}<6.75 \mathrm{e}-6 \mathrm{~g} / \mathrm{cm}^{2}$ there was no visible influence of mucilage on capillary rise; hence the original contact angle of the used glass plates was unaltered. A minimum contact angle of $30^{\circ}$ was chosen for the untreated sandy soil based on the measurements of Moradi et al. (2012), who measured the contact angle of the same sands used here - i.e. we took the values of the contact angle far from the root surface where exudates did not alter the soil wettability. These observations gave a range of possible values for the curve $\alpha\left(C_{S}\right)$. The semi-solid line shows the curve $\alpha\left(C_{S}\right)$ that gave the best fit between the pore-network model and the neutron radiography experiments. The relation consists of 


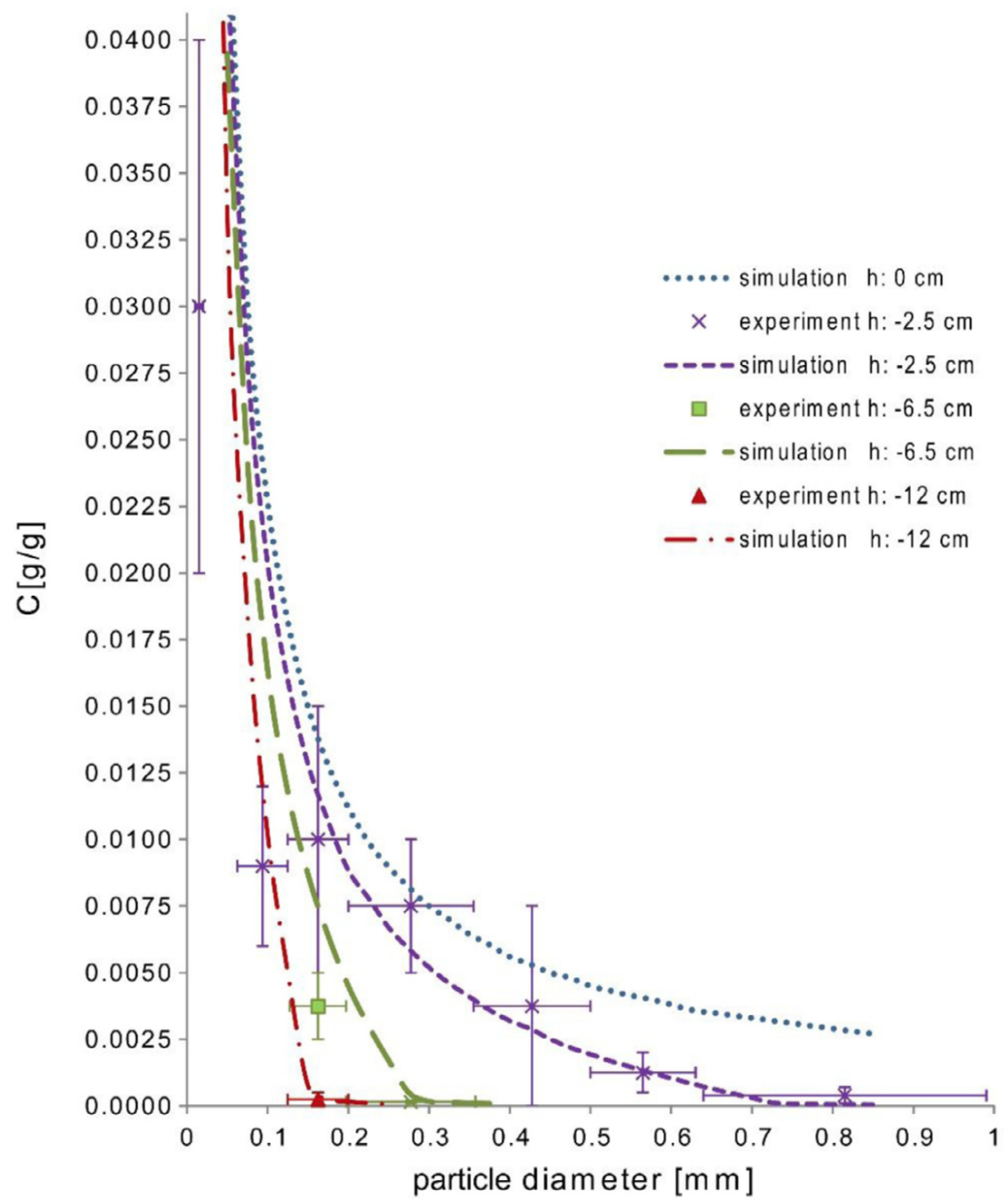

Figure 3.6: Mucilage concentration at the percolation threshold $\left(C_{t h}\right)$ [g of mucilage per g of dry soil] as a function of soil particle diameter for varying matric potentials (h). The lines show the critical mucilage concentrations derived from the pore-network simulations. The points with error bars show the experimental results for different soil particle diameters. 


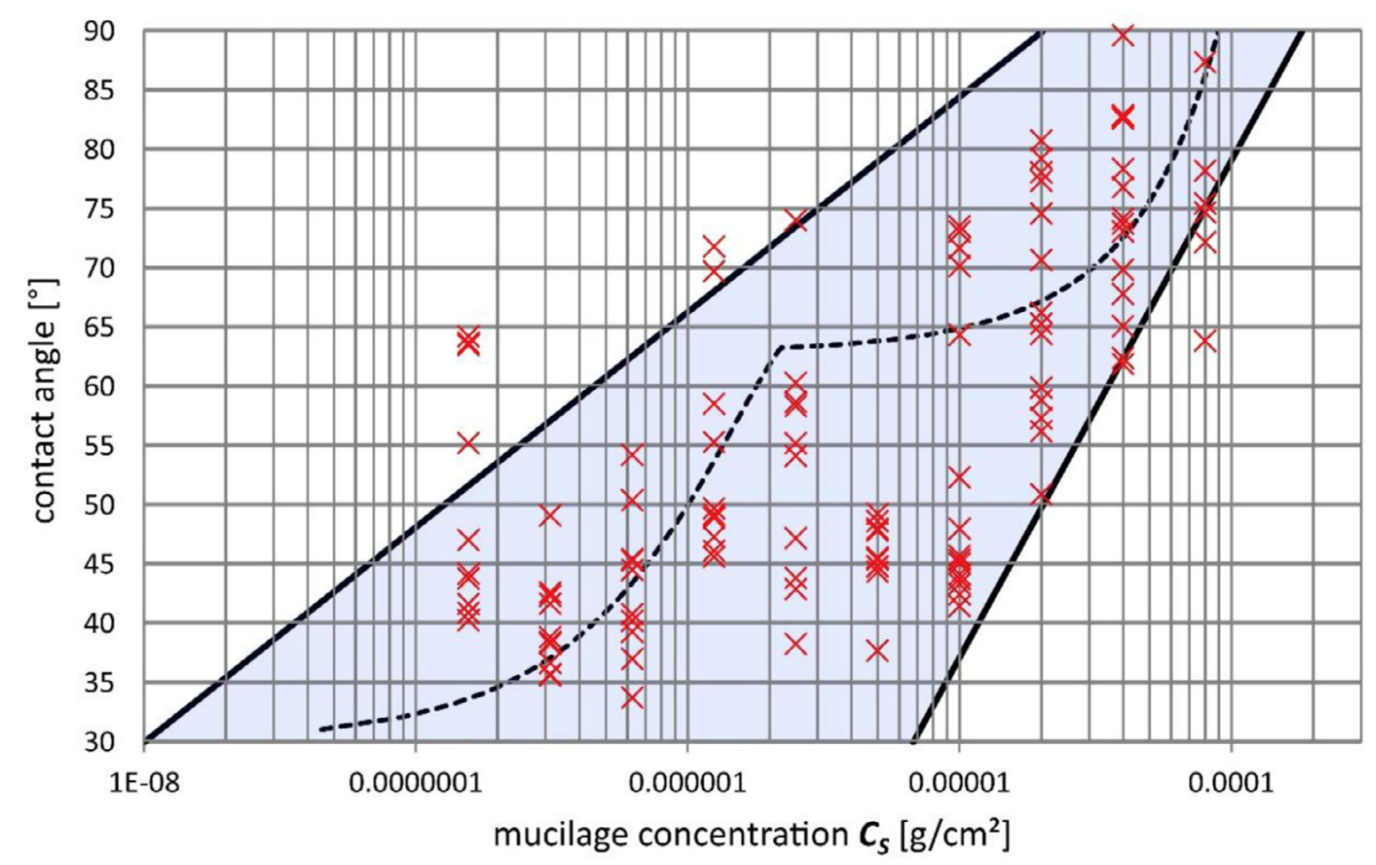

Figure 3.7: Measured and fitted relations between mucilage concentration per surface area $\left(C_{S}\right)$ and contact angle $(\alpha)$. Contact angles measured with the sessile drop methods are marked with crosses. The dashed line gave the best fit between observed and simulated $C_{t h}$.

two polynomial functions:

$$
\begin{gathered}
\alpha=-4.05 \cdot 10^{12} C_{S}^{2}+2.40 \cdot 10^{7} C_{S}+30 \text { for } 0 \leq C_{S} \leq 2.2 \cdot 10^{-6} \mathrm{~g} / \mathrm{cm}^{2} \\
\alpha=1.21 \cdot 10^{9} C_{S}^{2}+1.96 \cdot 10^{5} C_{S}+62.85 \text { for } C_{S}>2.2 \cdot 10^{-6} \mathrm{~g} / \mathrm{cm}^{2}
\end{gathered}
$$

The measurements with the sessile drop method (crosses) showed a similar trend, with contact angles of $35^{\circ}-45^{\circ}$ at $C_{S}=0.00015625 \mathrm{mg} / \mathrm{cm}^{2}$ and contact angles up to $90^{\circ}$ and larger for $C_{S}$ of $0.08 \mathrm{mg} / \mathrm{cm}^{2}$. In total 9 of 15 measurements at a concentration of $0.08 \mathrm{mg} / \mathrm{cm}^{2}$ resulted in contact angles $>90^{\circ}$, with a maximum of $109^{\circ}$. In the figure we showed only the values $\leq 90^{\circ}$, because for our model contact angles equal to $90^{\circ}$ or bigger than $90^{\circ}$ have the same effect.

These results show that the percolation model was able to predict the critical mucilage concentration at which the rhizosphere turned impermeable. The fitted curve $\alpha\left(C_{S}\right)$ felt well in the range of the observations. The effect of particle size and matric potential on $C_{t h}$ was well described by the model.

For soils with a particle diameter $d<0.125 \mathrm{~mm}$ the model over-estimated $C_{t h}$ by a factor of 2-3. For particle diameters of $d=0-0.02 \mathrm{~mm}$, the experimental $C_{t h}$ was $0.094 \mathrm{~g} / \mathrm{g}$ and the 
simulated $C_{t h}$ was $0.02 \mathrm{~g} / \mathrm{g}$. For particle diameters of $d=0.063-0.125 \mathrm{~mm}$, the experimental $C_{t h}$ was $0.03 \mathrm{~g} / \mathrm{g}$, while the simulated $C_{t h}$ was $0.09 \mathrm{~g} / \mathrm{g}$. This overestimation may be caused by the swelling of mucilage in the fine pores and the consequent pore clogging, as suggested by Hallett et al. (2003). In fact, assuming that the swelling of mucilage follows a diffusion behaviour, we can expect that the time needed to clog a pore goes like $t=r^{2} / D$ where $r$ is the pore radius and $D$ is a diffusion coefficient describing the mucilage swelling. In soils with fine particles the time needed to clog the pores would therefore be much shorter than in coarse textured soils. Based on this argument, we expect that pore clogging due to mucilage swelling occurred during the experiments with the fine textured soils. Due to the joint effect of hydrophobicity and pore clogging, less mucilage was actually needed to impede the flow in the fine textured soils. To describe the effect of pore clogging due to mucilage swelling, the kinetics of the mucilage expansion through the pores should be included in the model. In this paper, we neglected these dynamic effects and focused on the initial rewetting phase.

The model was compared to experiments with a silty soil and quartz sands of comparably narrow grain size distributions. However, natural soils have broader and often multi-modal pore size distributions. In such cases, the spatial distribution of mucilage becomes important. For instance, the possibility that mucilage is uniformly distributed on all pore surfaces or that, more likely, it is preferentially distributed in small or large pores would affect the quantity of mucilage needed to impede the water flow at varying matric potentials. Interestingly, Albalasmeh and Ghezzehei (2014) observed a preferential distribution of polygalacturonic acid (an analogue of mucilage) in the contacts between spherical particles that were let dry. Similarly, it could be expected that a higher amount of mucilage is deposited in the small pores during drying. However, our current understanding of pore-scale mechanism of mucilage deposition at varying soil moisture conditions is still limited and it deserves further experimental and modelling studies.

Some of the assumptions of our model were clear simplifications of real porous media. We neglected water flow in pore corners and we used a cubic lattice, which has a coordination number of 6 , while more realistic pore-network models could allow variable coordination numbers (Valvatne and Blunt, 2004). Additionally, we focused on the initial phase of the rewetting process and we just checked if water could flow or not through the porous medium, without simulating the time dynamics of the rewetting.

Further improvements of the model should include the implementation of a grain size distribution, for example using a site-bond percolation approach and distributing the different 
pore radii across the lattice. Secondly, the effects of the relation between pore radius and mucilage quantity should be investigated, either assuming a uniform or a preferential mucilage distribution in pores of a given class. By introducing a more realistic pore geometry, for instance using triangular pores (Blunt, 2001), the model could be extended to simulate water and mucilage interactions in the pore corners, where probably mucilage is deposited as the soil dries. Also, tomography based three- dimensional images of the rhizosphere would provide a more precise description of the process. Recently, X-ray CT has been successfully used to reconstruct the pore morphology in the rhizosphere (Aravena et al., 2010; Keyes et al., 2013, Schmidt et al., 2012, Daly et al., 2015). These advancements in pore-scale imaging offer new avenues to relate the pore-scale physics of the rhizosphere to root water uptake and soil-plant interactions.

\subsection{Conclusions}

We developed a simple pore-network model of water percolation through the rhizosphere. The model is capable of reproducing the dual behaviour of the rewetting of soils mixed with mucilage. When the mucilage concentration is below a given threshold $C_{t h}$ water can easily cross the rhizosphere. When the mucilage concentration is higher than $C_{t h}$ the soil becomes water repellent, impeding the water flow. Near the critical mucilage concentration, the infiltration front becomes irregular and shows fingering.

The simplicity of the model allows highlighting two principle factors controlling the wettability of the root-soil interface: the particle size and the mucilage concentration (gram of mucilage per gram of soil). The radiographs shown at the beginning of this paper (Fig. 3.1) suggest that the rhizosphere of lupines in sandy soils is near the percolation threshold, where small variations in mucilage exudation would mean a big change in the wettability of the rhizosphere. It is tempting to think that the amount of mucilage exuded is functional to maintaining the rhizosphere near the percolation threshold, so that the roots can efficiently adjust the hydraulic conductivity of the root-soil interface. To confirm this hypothesis, it would be interesting to study whether plants adapted to different soil textures exude a different amount of mucilage, or, alternatively, a mucilage containing a different amount of hydrophobic compounds. The simplicity of this model allows addressing in a quantitative way such complex ecological questions. 


\title{
4 Drying of mucilage causes water repellency in the rhizo- sphere of maize: measurements and modelling ${ }^{5}$
}

written by Mutez Ali Ahmed, Eva Kröner, Pascal Benard, Mohsen Zarebanadkouki, Anders Kaestner, and Andrea Carminati, published in Plant and Soil (Ahmed et al., 2015c)

\begin{abstract}
Background and Aims Although maize roots have been extensively studied, there is limited information on the effect of root exudates on the hydraulic properties of maize rhizosphere. Recent experiments suggested that the mucilaginous fraction of root exudates may cause water repellency of the rhizosphere. Our objectives were: 1) to investigate whether maize rhizosphere turns hydrophobic after drying and subsequent rewetting; 2) to test whether maize mucilage is hydrophobic; and 3) to find a quantitative relation between rhizosphere rewetting, particle size, soil matric potential and mucilage concentration.

Methods Maize plants were grown in aluminum containers filled with a sandy soil. When the plants were three-weeks-old, the soil was let dry and then it was irrigated. The soil water content during irrigation was imaged using neutron radiography. In a parallel experiment, ten maize plants were grown in sandy soil for five weeks. Mucilage was collected from young brace roots growing above the soil. Mucilage was placed on glass slides and let dry. The contact angle was measured with the sessile drop method for varying mucilage concentration. Additionally, capillary rise experiments were performed in soils of varying particle size mixed with maize mucilage. We then used a pore-network model in which mucilage was randomly distributed in a cubic lattice. The general idea was that rewetting of a pore is impeded when the concentration of mucilage on the pore surface $\left(\mathrm{g} \mathrm{cm}^{-2}\right)$ is higher than a given threshold value. The threshold value depended on soil matric potential, pore radius and contract angle. Then, we randomly distributed mucilage in the pore network and we calculated the percolation of water across a cubic lattice for varying soil particle size, mucilage concentration and matric potential.
\end{abstract}

Results Our results showed that: 1) the rhizosphere of maize stayed temporarily dry after irrigation; 2) mucilage became water repellent after drying. Mucilage contact angle increased with mucilage surface concentration (gram of dry mucilage per surface area); 3)

\footnotetext{
${ }^{5}$ E.K. developed the analytical estimation of the percolation threshold and partly prepared the capillary rise experiment.
} 
Water could easily cross the rhizosphere when the mucilage concentration was below a given threshold. In contrast, above a critical mucilage concentration water could not flow through the rhizosphere. The critical mucilage concentration decreased with increasing particle size and decreasing matric potential.

Conclusions These results show the importance of mucilage exudation for the water fluxes across the root-soil interface. Our percolation model predicts at what mucilage concentration the rhizosphere turns hydrophobic depending on soil texture and matric potential. Further studies are needed to extend these results to varying soil conditions and to upscale them to the entire root system.

\subsection{Introduction}

Water scarcity is considered a major threat and a challenge that must be overcome in the twenty-first century. Modern agricultural production relies on the high availability of input resources like fertilizer and water (Lynch and Brown, 2012). However, it is widely understood that a limited water supply is one of the largest impediments to food production worldwide. Therefore, increasing plant drought tolerance and improving the capacity of agricultural plants to extract water from the soil are fundamentally imperative to sustain the increasing food demand caused by modern population growth trends.

A recently suggested approach to enhance crop productivity is based on plant-soil feedbacks that can improve the capacity of plant roots to extract water from the soil (Sposito, 2013). Extraction of water from soils depends on several root traits, such as: root architecture (Wasson et al., 2012), root depth (Tron et al., 2015), internal axial and radial conductivity and the conductance of the shortest paths that links the roots to the shoot base (Lobet et al., 2014); capacity of roots to explore the heterogeneous soil resources and reach the subsoil (Lynch and Wojciechowski, 2015), and root-shoot signaling (Huber et al., 2014). An alternative strategy to increase the capture of water from the soil consists in modifying the environment where the roots grow and interact with, the rhizosphere.

The rhizosphere is the soil in the vicinity of the roots that is modified by the roots in several ways. Root growth compacts the soil and decreases the rhizosphere porosity (Aravena et al., 2010). Root shrinking during drying creates gaps between soil and roots (Carminati et al. 2009). Repeated drying and wetting cycles in the rhizosphere increase soil aggregation (Watt et al., 1994). Indeed, an increasing number of studies on soil structure, soil aggregation and water distribution around the roots showed that the physical properties of the rhizosphere 
differ in several ways from those of the adjacent bulk soil (Watt et al., 1994, Young, 1995; Carminati et al., 2010; Moradi et al., 2011).

An additional process affecting the rhizosphere physicochemical properties is the exudation of mucilage (Carminati and Vetterlein, 2013). Mucilage is mainly secreted at the root tips. It is primarily composed of polysaccharides and small fraction of lipids Read et al., 2003). A variety of functions have been attributed to mucilage: maintenance of a good contact between roots and soil particles, reduction of friction for root penetration, facilitation of root water uptake by increasing the rhizosphere water content, and avoidance of root tissue dehydration while the root tip is emerging (McCully, 1995; Hallett et al., 2003, Carminati et al., 2011; Ahmed et al., 2014).

Mucilage from maize (Zea mays L.) roots, in particular, was shown to have a remarkable ability to swell and adsorb water: fully hydrated mucilage can contain water up to 1000 times its dry weight (McCully and Boyer, 1997). The authors then tested the potential role of mucilage and its large water-holding capacity in protecting roots from drought. They found that mucilage loses most of its water at water potentials less negative than -0.01 MPa and concluded that mucilage water content, per se, does not play a significant role in drought protection. However, the remaining water at $-0.01 \mathrm{MPa}$, which is around 50 times its dry weight, would increase the gravimetric water content in the rhizosphere by about $5 \%$ (Carminati, 2012). Furthermore, Carminati et al. (2011) suggested that such an increase in water holding capacity of the rhizosphere, especially when the soil is dry, could limit the drop in the hydraulic conductivity at the root surface hereby maintaining the hydraulic contact between soil and roots. Recently, we showed that mucilage exuded by chia seeds (Salvia hispanica L.) favors root water uptake in dry soil (Ahmed et al., 2014) and increases the water holding capacity of a sandy soil at any water potential (Kroener et al., 2014b).

Besides the capacity to absorb water, mucilage exudation may also cause water repellency of the rhizosphere (Carminati et al., 2010; Moradi et al., 2012). Carminati et al. (2010) showed that the water content in the rhizosphere of lupine (Lupinus albus L.) was higher than in the bulk soil during a period of active transpiration. However, after the soil was dried and the samples were subsequently irrigated, the rhizosphere remained markedly drier than the bulk soil. It took approximately 2 days for the rhizosphere to become wet again. Moradi et al. (2012) measured contact angles higher than $90^{\circ}$ in the rhizosphere of lupine (Lupinus albus L.). Carminati and Vetterlein (2013) suggested that such a dynamic effect of mucilage on the rhizosphere water content could be considered as a plant strategy to regulate what part 
of the root system has facilitated access to water and what part becomes disconnected from the soil. For instance, fresh and hydrated mucilage may facilitate water uptake of young root segments, while dry and water repellent mucilage may isolate the old root segments from the drier soil regions.

In this manuscript, we focus on the water dynamics in the rhizosphere of maize during the rewetting phase. We aimed to experimentally verify the hypothesis that mucilage exudation causes water repellence in the rhizosphere of maize. Additionally, we intended to quantitatively relate the rewetting kinetics of the rhizosphere to mucilage concentration, soil particle size and soil water potential. Our specific objectives were: 1) to find whether maize rhizosphere turns hydrophobic after drying and subsequent rewetting; 2) to test if mucilage becomes hydrophobic upon drying; and 3) to estimate in what soil textures the rhizosphere of maize is prone to water repellency.

To address these points we: 1) used neutron radiography to monitor the water content in the rhizosphere of maize during drying and after rewetting; 2) measured the contact angle of maize mucilage at varying surface concentration (gram of dry mucilage per surface area); 3) performed capillary rise experiments with soils of varying particle size mixed with maize mucilage; 4) developed a model of water percolation across the rhizosphere at the pore scale.

\subsection{Materials and methods}

Soil and plant preparation We used a sandy soil collected near Göttingen, Germany. The soil was sieved to a particle size smaller than $2 \mathrm{~mm}$ and then filled into 10 pots $(15 \mathrm{~cm}$ diameter, $20 \mathrm{~cm}$ height). Maize seeds were soaked in $10 \% \mathrm{H}_{2} \mathrm{O}_{2}$ solution for 1 minute and then germinated on moist filter paper for 48 hours. The seedlings were then planted at a depth of $0.5 \mathrm{~cm}$ into the soil (one seed per pot). The upper soil layers were covered with a $1 \mathrm{~cm}$ layer of quartz gravel (3 $\mathrm{mm}$ diameter) to reduce evaporation. The plants were grown with a daily cycle of $14 \mathrm{~h}$ light and $10 \mathrm{~h}$ darkness, with a light intensity of $500 \mu \mathrm{mol} \mathrm{m}{ }^{-2}$ $\mathrm{s}^{-1}$, day/night temperatures of $22 / 19^{\circ} \mathrm{C}$, and relative humidity of $60 \%$. The aim of this experiment was to measure the contact angle of mucilage collected from maize plants.

In addition, we grew 10 maize plants in aluminum containers for neutron radiography experiments. The containers $(30 \mathrm{~cm} \times 15 \mathrm{~cm} \times 1 \mathrm{~cm})$ were comprised of two aluminum sheets held together at the edges by three 1-cm-thick bars. The small thickness of the container is a requirement of neutron radiography. A thicker layer of wet soil would increase neutron scattering, making less accurate the quantification of water content. The aluminum containers 


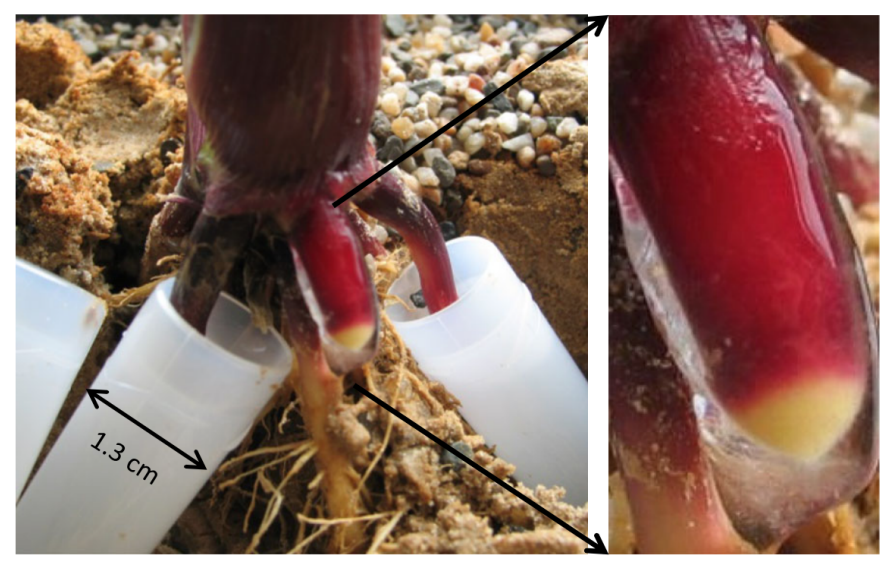

Figure 4.1: Brace roots of a 5-week-old maize plant. Roots were kept in plastic tubes filled with water for $24 \mathrm{~h}$ to keep mucilage fully hydrated.

were laid horizontally and the detachable top sheet was removed. The sandy soil was then poured into the containers through a $2 \mathrm{~mm}$ sieve in order to minimize formation of soil layers. The top sheet of each container was then closed, and the containers were turned vertically and gently shaken to achieve a stable packing. The resulting bulk density was approximately $1.45 \mathrm{~g} \mathrm{~cm}^{-3}$. The aim of this experiment was to find out whether maize rhizosphere turns hydrophobic after drying and subsequent rewetting.

Mucilage collection Mucilage was collected from the emerging brace roots of five-week-old maize plants. The brace roots had a diameter of 3.5-4 $\mathrm{mm}$. The brace roots were immersed in plastic cylinders filled with water for 24 hours (Fig. 4.13). The tubes were connected in the morning and the roots were left in water for the whole day and night. The next morning the cylinders were removed and a blob of mucilage covered the root segment immersed in water (Fig. 4.1b). Mucilage was removed from the roots using a syringe.

Contact angle measurements The contact angle (CA) of mucilage was measured for varying mucilage concentrations (gram of dry mucilage per surface). Different amounts of mucilage collected from maize plants were spread on glass slides having a surface of $4 \mathrm{~cm}^{2}$. Then mucilage was let dry on the glass slides. We measured the CA for six different mucilage concentrations.

The contact angle was determined with the sessile drop method, by monitoring the placement and subsequent behavior of a drop of deionized water (SDM, Goebel et al. (2013)) using a CCD-equipped CA microscope (Drop Shape Analyzer DSA25S; KRÜSS GmbH). The drop 
volume was $2 \mu \mathrm{l}$. The contact angle of each drop is given as the mean of the left and the right side in the images (Fig. 4.4).

Neutron radiography experiments Neutron imaging is being increasingly used to image water and root distribution in soil because of its high sensitivity to hydrous materials (Oswald et al., 2008; Moradi et al., 2009; Carminati et al., 2010; Moradi et al., 2011; Ahmed et al., 2015a). With neutron radiography, a parallel neutron beam propagates through the sample and the transmitted neutrons behind the sample are detected using a scintillator. The scintillator converts the neutrons into visible light, which is acquired using a CCD camera that provides the information in the form of a digital image. The detected image carries the information on the thickness and composition of the sample, this is described by Beer-Lamberts law.

Our experiments were performed at the ICON beam-line of the Paul Scherrer Institute (PSI), Switzerland (Kaestner et al. 2011). We used a sCMOS camera with an array of $2160 \times 2560$ pixels, resulting in a field of view of $13.3 \times 16 \mathrm{~cm}$ and with a pixel size of $0.062 \mathrm{~mm}$. The measurements with neutron radiography started three weeks after seed germination. The samples were let dry to a water content of $0.04-0.05 \mathrm{~cm}^{3} \mathrm{~cm}^{-3}$ and then they were irrigated. The samples were imaged before irrigation and 30 minutes after irrigation.

Capillary rise experiment The capillary rise experiments were conducted with soils of various particle size distributions $(<20,63-200,200-500,360-630,630-1000 \mu \mathrm{m})$. To reproduce an analogue of the rhizosphere, we mixed soils of different particle sizes with maize mucilage. The mucilage was collected from maize roots as described above. The mucilage-soil mixture was dried for about 24 hours at $40^{\circ} \mathrm{C}$. To avoid accumulation of dry mucilage on the surface, the mixture was spread on a large surface with the height of the mixture being around $1 \mathrm{~mm}$. The mucilage-sand mixture formed a crust which was then gently crushed into individual grains.

The experimental set-up consisted of thin rectangular containers with a cross section of $1 \mathrm{~cm}$ filled with three soil layers. The lower part of each sample was filled with an uncoated sandy soil. The second layer was filled with the soil-mucilage layer which had a concentration of $0.5 \%$ ( $\mathrm{g}$ of dry mucilage per $\mathrm{g}$ of dry soil). This layer represented the rhizosphere. On the top, we added another layer of uncoated sand. The samples were initially dry. Then the lower part of the sample was immersed in water until the water table reached a given height below the second layer (representing the rhizosphere). This height corresponded to 
(minus) the matric potential of the water rewetting of the rhizosphere. The capillary rise in the samples was monitored using neutron radiography.

\subsection{Conceptual model}

The percolation approach: We used a simple pore-network model of water percolation through a water repellent soil. The general idea is that mucilage covers a fraction of the pores making them susceptible to water repellency. The higher is the amount of mucilage, the higher becomes the fraction of pores turning water repellent. When the fraction of pores turning water repellent increases above a critical value, then water flow is impeded and the soil becomes hydrophobic.

This problem of varying the number of connected/disconnected pores in relation to the flow through porous media has been elegantly addressed using the percolation theory. The concept of the percolation theory has been used in a number of approaches to predict transport properties of a porous media by a description of relevant processes at the microscopic scale (Berkowitz and Balberg, 1992, 1993). A review of applications can be found in Berkowitz and Ewing (1998). Hunt (2004) predicted the critical moisture content for capillary flow employing the percolation approach. Steenhuis et al. (2005) employed the concept of percolation theory to demonstrate the hydrological influence of hydrophobic grains in a soil. They demonstrated how a few hydrophobic grains can make a soil water repellent.

We developed a simple percolation model to predict how the microscopic distribution of mucilage at the pore scale affects the rewetting of maize rhizosphere. We focused on the initial phase of the rewetting process and we did not consider the rehydration and swelling of the gel. This simplification is justified by the different time-scales of the two processes: the initial water flow through the rhizosphere has a time scale of seconds to a few minutes, while the rehydration of mucilage has a time scale of hours.

An illustration of the model is shown in Fig. 4.2. Dry mucilage is randomly distributed on the surface of the soil particles. The soil pores that have a higher mucilage concentration, defined as gram of dry mucilage per soil surface, turn hydrophobic. After irrigation these pores are not immediately rewetted and temporarily impede the water flow. On the contrary, the pores that are not covered by mucilage or that have a lower mucilage concentration than $C$ are rewetted and let the water flow. We assume that the contact angle depends on mucilage concentration. We expect that the contact angle increases with mucilage concentration per unit soil surface area. 


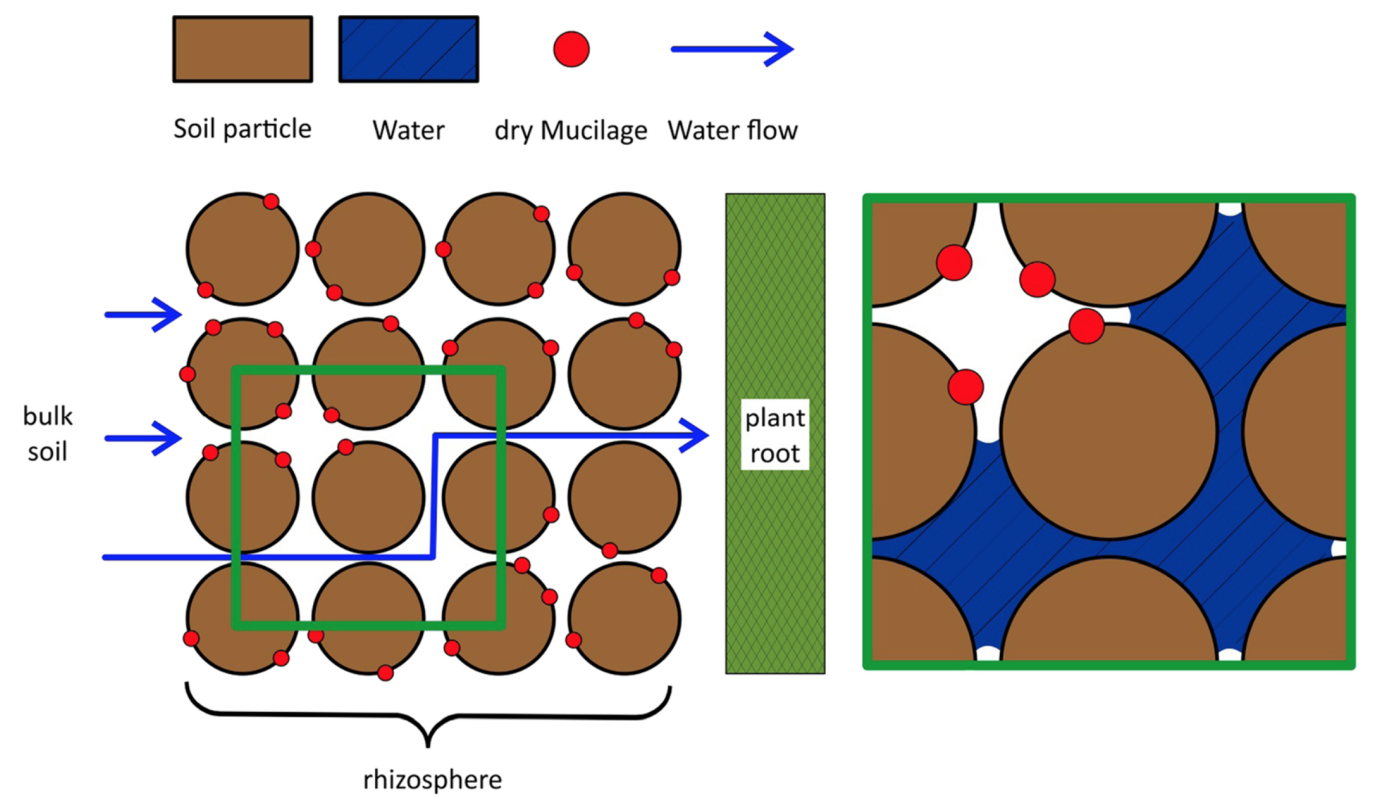

Figure 4.2: Illustration of the effect of mucilage on water flow in the rhizosphere. This is a 2D sketch but the model accounts for the 3D extension of the rhizosphere. After drying mucilage becomes hydrophobic and impedes the water flow in a fraction of the pores.

Kroener et al. (2015) showed experimentally that, for soil layers mixed with artificial mucilage collected from chia seeds (Salvia hispanica L.), there is a critical mucilage concentration above which water could no longer percolate across the layer. They used a simple percolation model to predict the mucilage concentration at percolation threshold as a function of soil particle size for saturated conditions with matric potential close to zero. In this manuscript, we extend the previous model to the unsaturated case and relate mucilage concentration and particle size to the water potential at percolation threshold.

In our study we applied a simple site percolation model where all soil particles are represented as spheres of radius $r_{\text {part }}$ arranged in a cubic lattice. The number of particles per unit of volume $N$ (particles $\mathrm{cm}^{-3}$ ) is related to the bulk soil density $\rho_{b u}$ and soil mineral density $\rho_{m i}$ :

$$
N=\frac{1}{\frac{4}{3} \pi r_{p a r t}^{3}} \frac{\rho_{b u}}{\rho_{m i}}
$$

Assuming a cubic packing of sphere, the relation between the pore radius (biggest sphere that can be put into the pore space between the particles) and the particle radius is given by:

$$
r_{\text {pore }}=(\sqrt{3}-1) r_{\text {part }}=0.73 r_{\text {part }}
$$

Note that the representation of pores and particles as spheres means that the entire volume 
is not completely represented as either pore or particle from a geometrical point of view. However, for our estimation this approximation is sufficient. In a cubic lattice the number of particles per unit volume $N$ (particles $\mathrm{cm}^{-3}$ ) is equal to the number of pore bodies of radius $r_{\text {pore }}$.

We assume that the total concentration of dry mucilage $C\left[\mathrm{~g} \mathrm{~cm}^{-3}\right]$ is normally distributed inside the pores. The average mass of mucilage per pore $C N^{-1}\left[\mathrm{~g}\right.$ pore $\left.{ }^{-1}\right]$ is:

$$
\frac{C}{N}=C \frac{4}{3} \pi r_{\text {Part }}^{3} \frac{\rho_{m i}}{\rho_{b u}}
$$

Dividing by the pore surface $A_{\text {pore }}=4 \pi r_{\text {pore }}^{2}$ gives the average dry mass of mucilage per pore surface $c_{\text {pore }}\left[\mathrm{g} \mathrm{cm}^{-2}\right]$ :

$$
c_{\text {pore }}=\frac{C}{A_{\text {pore }} N}=\frac{C}{3} \frac{r_{\text {part }}^{3}}{r_{\text {pore }}^{2}} \frac{\rho_{\text {mi }}}{\rho_{b u}}
$$

Using Equation (4.2) gives:

$$
c_{\text {pore }}=\frac{C}{1.6} r_{\text {part }} \frac{\rho_{m i}}{\rho_{b u}}
$$

For a site pore network model based on a cubic 3-dimensional lattice the percolation threshold is at $31.2 \%$ (Stauffer, 1985). That means water can flow through the soil only if at least $31.2 \%$ of the pores are permeable for water flow. We use the Young-Laplace equation to calculate at what matric potential $h$ a pore with a given contact angle and pore radius can be rewetted:

$$
h>\frac{-2 \gamma \cos (\alpha)}{r_{\text {pore }} \rho_{w} g}=\frac{-2 \gamma \cos (\alpha)}{0.73 r_{\text {part }} \rho_{w} g}
$$

where $\gamma=0.073 \mathrm{mN} \mathrm{cm}^{-1}$ is the surface tension of water in air at $20^{\circ} \mathrm{C}, \rho_{w}=1 \mathrm{~g} \mathrm{~cm}^{-3}$ is the density of water, $g=9.81 \mathrm{mN} \mathrm{g}^{-1}$ is the gravity constant, and $\alpha$ is the contact angle between air, water and a solid surface covered with a given concentration of mucilage $c\left(\mathrm{~g} \mathrm{~cm}^{-2}\right) . \alpha$ depends on the mucilage concentration in the pore $(c)$. We obtained the relation $\alpha$ (c) between contact angle $\alpha$ and mucilage concentration $c$ from our contact angle measurements.

In our model we assume that the distribution of mucilage concentration in the pores has a Gaussian shape with mean $c_{\text {pore }}$ and standard deviation $\sigma$. For such a distribution the cumulative distribution function of pores with less concentration than $c$ is:

$$
F(c)=\frac{1}{2}\left[1+\operatorname{erf}\left(\frac{c-c_{\text {pore }}}{\sigma \sqrt{2}}\right)\right]
$$

At the percolation threshold $31.2 \%$ of the pores are permeable. In other words: if $c_{l}$ is the largest mucilage concentration of those pores that are still permeable then: $\mathrm{F}\left(c_{l}\right)=0.312$. Using Eq. 4.7 we obtain:

$$
c_{l}=c_{\text {pore }}+\sigma \sqrt{2} \operatorname{erf}^{-1}[2 \times 0.312-1]=c_{\text {pore }}-0.490 \sigma
$$




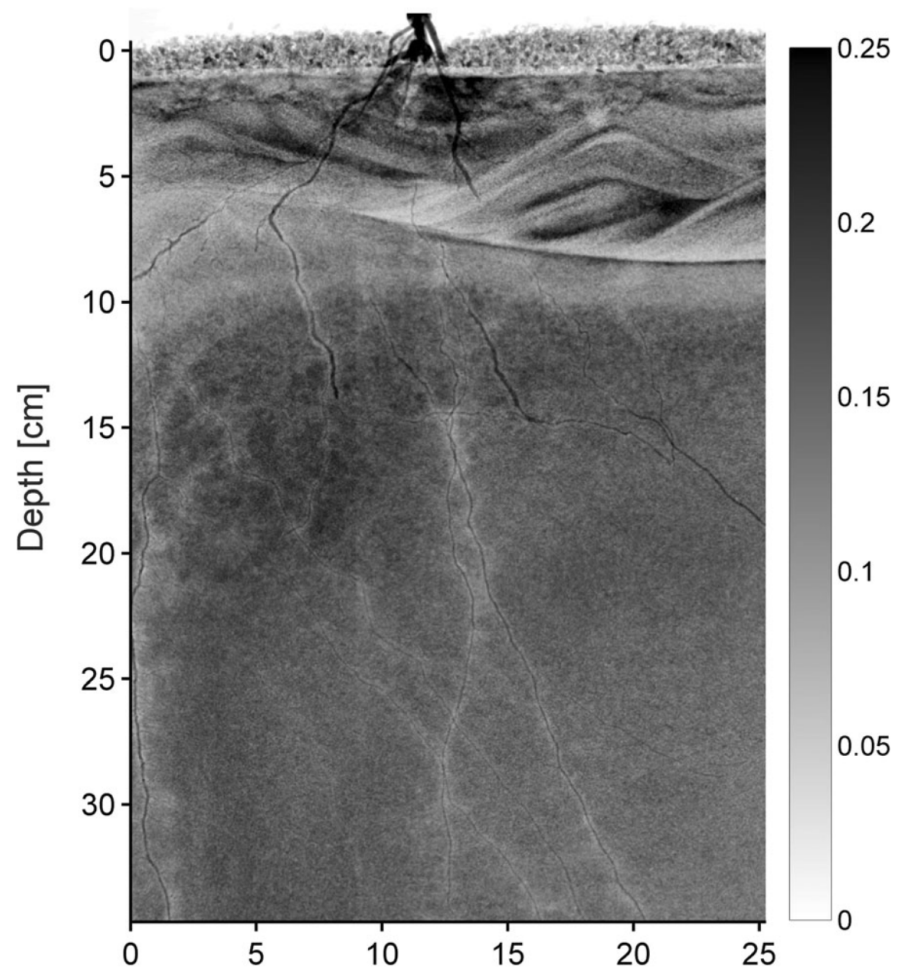

Figure 4.3: Neutron radiograph of water distribution around the roots of 3-weeks-old maize $30 \mathrm{~min}$ after irrigation. The grey values are proportional to the water content (dark=wet, bright $=$ dry). The figure shows that the rhizosphere of most roots appeared brighter than the bulk soil. This shows that maize rhizosphere remained dry after irrigation.

$$
c_{l}=0.472 C r_{\text {part }} \frac{\rho_{m i}}{\rho_{b u}}
$$

The relation given in Eq4.6 finally gives the water potential as a function of particle size and mucilage concentration at the percolation threshold:

$$
h=\frac{-2 \gamma \cos \left[\alpha\left(0.472 C r_{p a r t} \frac{\rho_{m i}}{\rho_{b u}}\right)\right]}{0.73 r_{p a r t} \rho_{w} g}
$$

\subsection{Results and Discussions}

We used neutron radiography to image the water distribution in the rhizosphere of maize roots that were irrigated after a drying cycle. Figure 4.3 shows the water content distribution in the root zone of a three-weeks-old maize 30 minutes after irrigation. The samples were irrigated from the top using a syringe. In the images, the water content is proportional to the gray values: i.e. dark means wet. The figure shows that the rhizosphere of most roots appeared 


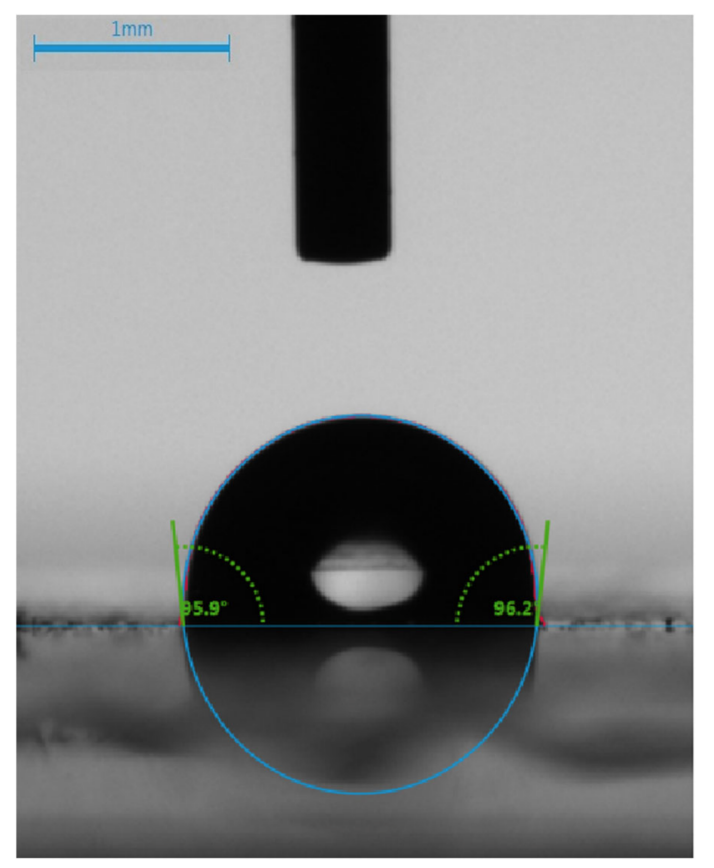

Figure 4.4: Contact angle of the dry maize mucilage on the glass slides. The contact angle was determined with the sessile drop method.

brighter than the bulk soil. This shows that maize rhizosphere remained dry after irrigation. The experiment was replicated 5 times. All samples showed the same behavior. Our results are in line with the observations of water repellence in the rhizosphere of lupine Carminati et al., 2010; Moradi et al., 2012). Carminati et al. (2010) showed that the water content in the rhizosphere of lupine (Lupinus albus L.) was higher than in the bulk soil during a period of active transpiration. However, after the soil was dried and the samples were subsequently irrigated, the rhizosphere remained markedly drier than the bulk soil. It took approximately 2 days for the rhizosphere to become wet again. More recently, Moradi et al. (2012) measured contact angles higher than $90^{\circ}$ in the rhizosphere of lupine (Lupinus albus L.). Carminati and Vetterlein (2013) suggested that the rhizosphere hydrophobicity was caused by mucilage exudation.

To verify this hypothesis, we measured the contact angle of mucilage collected from maize roots. Mucilage was collected from the young brace roots growing above the soil (Fig. 4.1). The collected mucilage was spread on glass slides having a surface area of $4 \mathrm{~cm}^{2}$. Then mucilage was let dry on the glass slides. Figure 4.4 shows an example of the measurements. The contact angle of maize mucilage was measured for varying mucilage concentrations (gram of dry mucilage per unit surface area). The relation between the contact angle and maize 


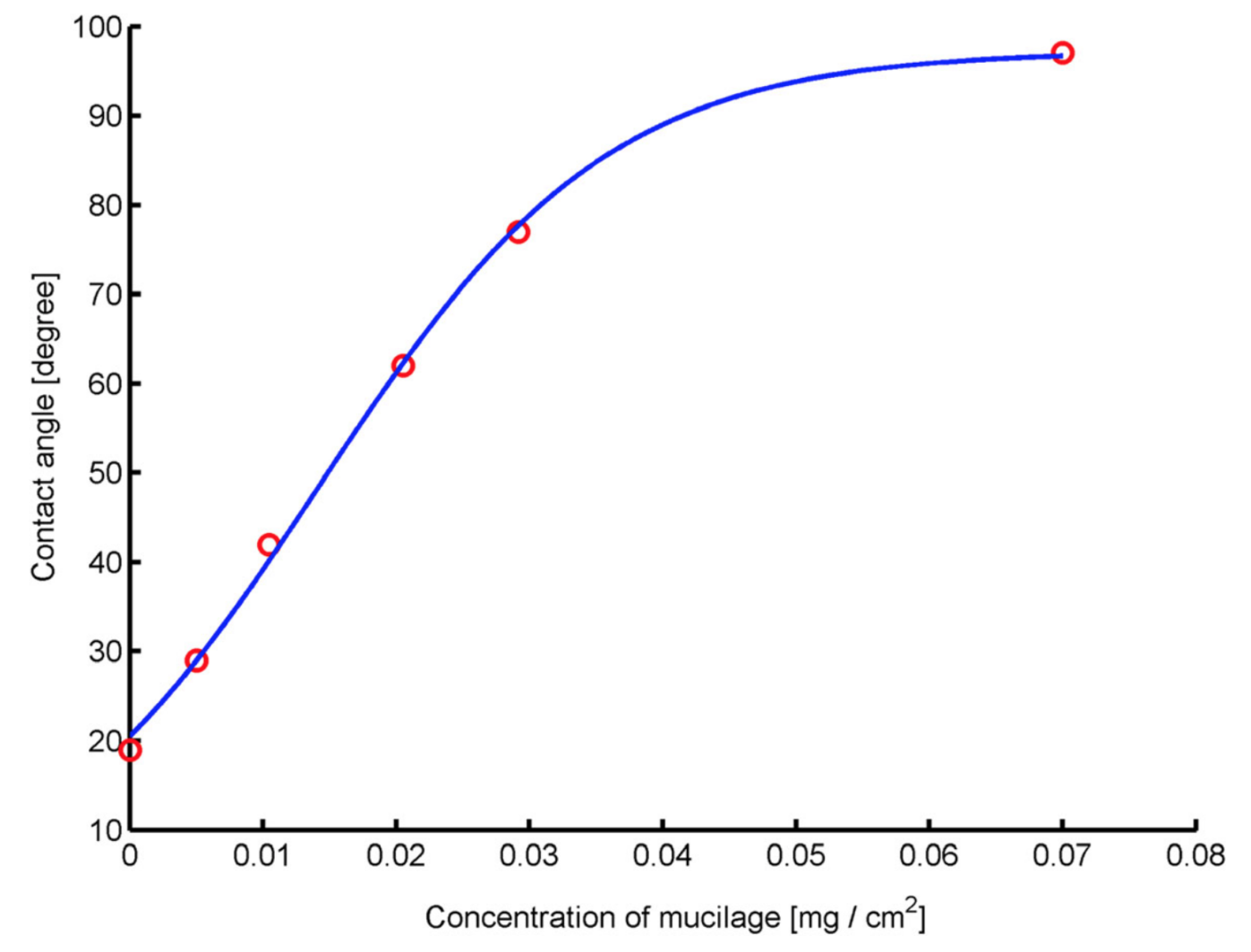

Figure 4.5: Contact angle of dry mucilage collected from maize plants. Mucilage was hydrophobic $\left(\mathrm{CA}>90^{\circ}\right)$ at higher mucilage concentration. The contact angle decreased for lower mucilage concentrations. The blue line shows the fitting of our model.

mucilage concentration is plotted in Fig. 4.4. The figure shows that maize mucilage became water repellent after drying. Mucilage contact angle increased with mucilage concentration (gram of dry mucilage per unit surface area).

Additionally, we performed capillary rise experiments in soils with five different particle sizes mixed with maize mucilage. The radiograph of the capillary rise experiment is shown in Fig. 4.6. The image shows the water content in the samples 5 minutes after irrigation from the bottom. The samples were initially dry. Then the lower part of the sample was immersed in water. The water content was derived by referencing the actual radiographs to the radiograph of the dry samples. The details of the image analysis are described elsewhere (Carminati et al. 2010). In the images, grey values are proportional to the water content i.e. dark means wet. The radiographs of the capillary rise experiments showed that for the finer soil textures $(<20 \mu \mathrm{m}, 60-200 \mu \mathrm{m})$ water could easily cross the rhizosphere layer, for particle sizes of $200-500 \mu \mathrm{m}$ water could still percolate through the layer without rewetting it 

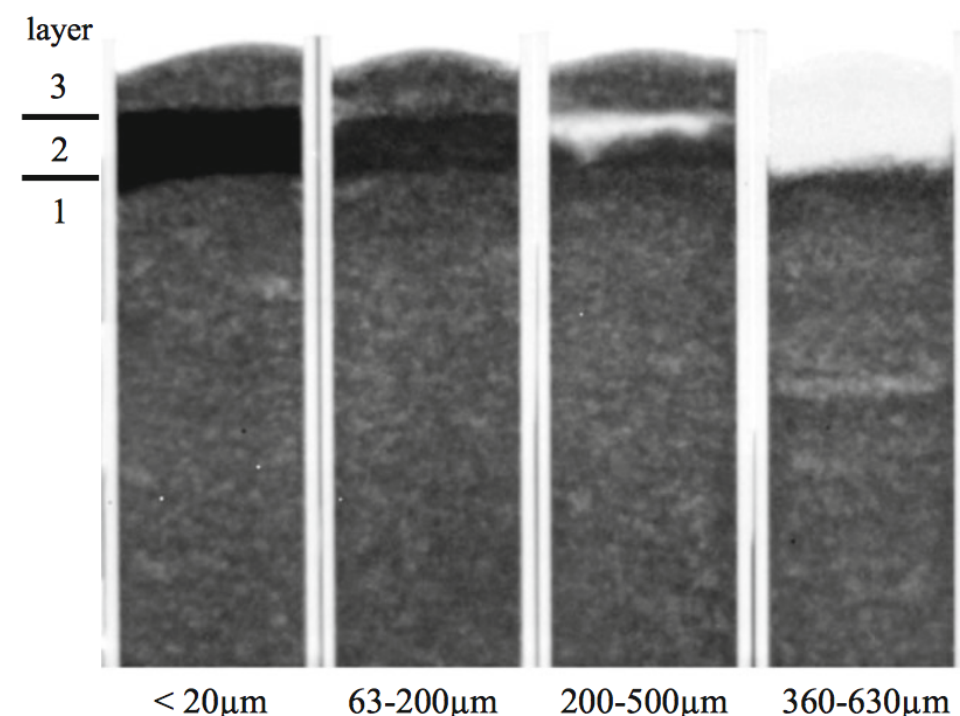

$63-200 \mu \mathrm{m}$

$200-500 \mu \mathrm{m}$

$360-630 \mu \mathrm{m}$

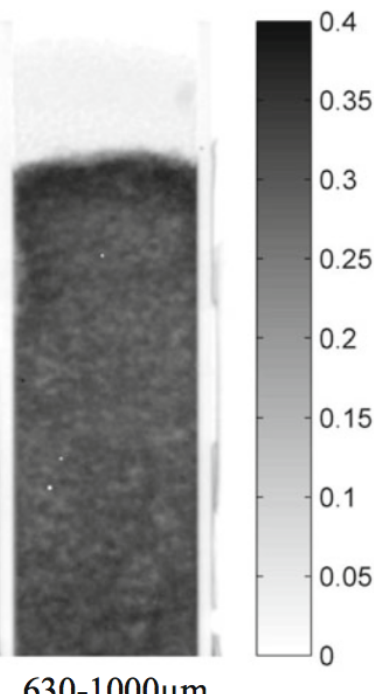

$630-1000 \mu \mathrm{m}$

Figure 4.6: Neutron radiographs of the capillary rise experiments in soil with different particle size $(<20,63-200,200-500,360-630,630-1000 \mu \mathrm{m})$. The lower part of each sample was filled with an untreated sandy soil. The second layer was filled with a soil- mucilage layer with a mucilage concentration of $0.5 \% \mathrm{~g}$ of dry mucilage per g of dry soil. This layer represented the rhizosphere. Then we added another layer of untreated sand. The figure shows that for particles sizes $<200 \mu$ m water could easily cross the rhizosphere, while for particle sizes $>360$ $\mu \mathrm{m}$ above the critical concentration water could no longer percolate through it. 


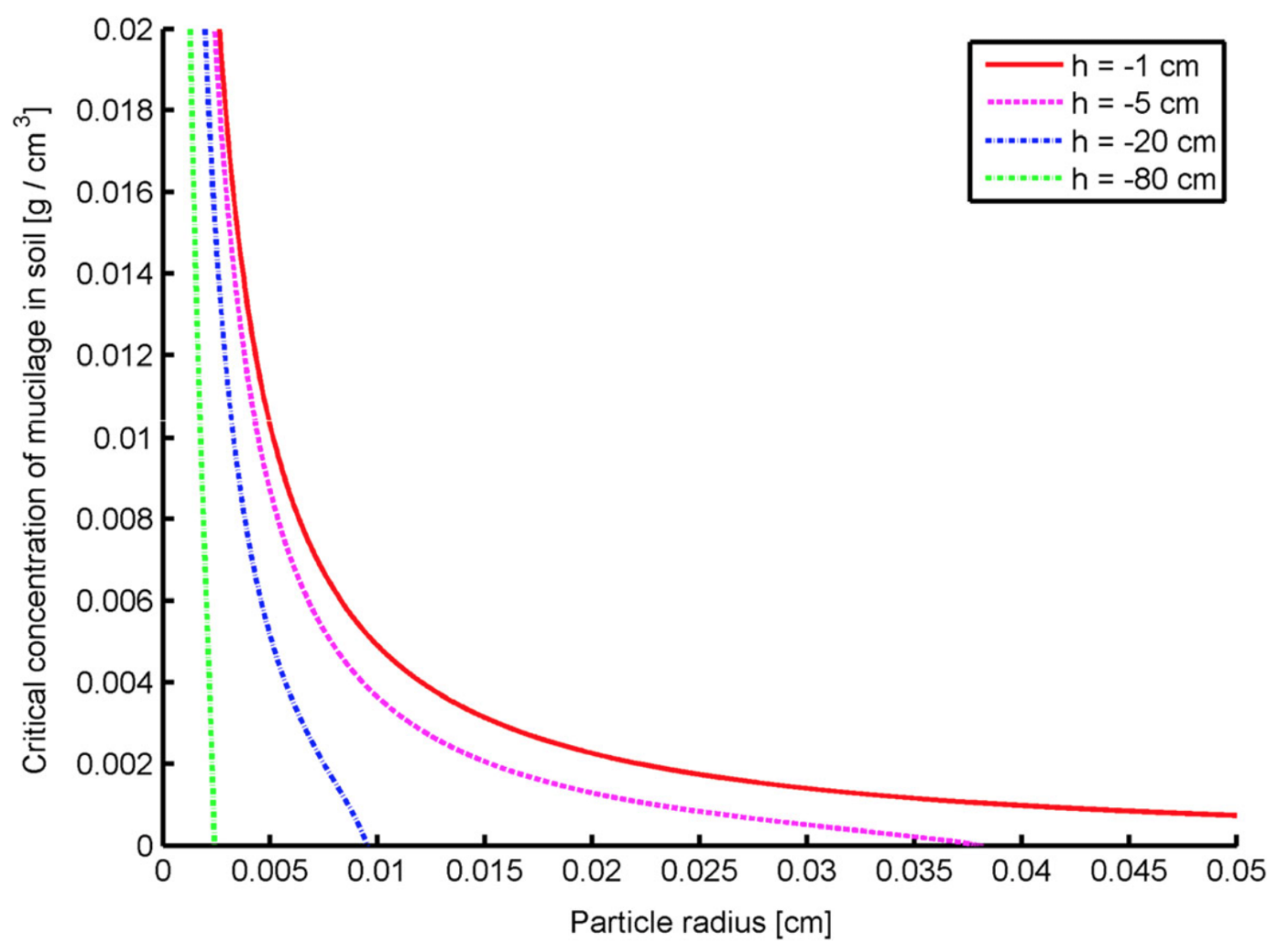

Figure 4.7: Maize mucilage concentration at the percolation threshold ( $\mathrm{g}$ of mucilage per $\mathrm{cm}^{3}$ of dry soil) as a function of soil particle diameter for varying matric potentials $(h)$. The figure shows the critical maize mucilage concentrations derived from the pore-network simulations

completely and for coarse textured layers $(360-630 \mu \mathrm{m}, 630-1000 \mu \mathrm{m})$ water could no longer percolate through this layer. This can only be explained when considering that the finer the soil texture, the larger the soil specific surface and the more mucilage is required to make the soil surface hydrophobic and to impede percolation of water through the treated layer.

We observed (data not shown) that the threshold mucilage concentration $(C)$ at the transition between the conductive to the non-conductive state depended not only on particle size but also on matric potential. Since repeating the capillary rise experiments in a systematic way for various matric potentials and particle sizes requires a considerable amount of maize mucilage which is practically very difficult to collect, we used our percolation model to quantitatively relate particle size, soil matric potential and mucilage concentration.

Figure 4.7 shows the relation between the concentration of maize mucilage at percolation threshold and soil particle size for varying matric potentials $(h)$. The effect of particle size and matric potential on mucilage concentration at the percolation threshold is well described by the model. The larger the soil particle diameter, the smaller is the specific soil surface and 
as a result less mucilage is required at the percolation threshold. Additionally larger particle diameters decrease the capillary forces, resulting in a lower critical mucilage concentration. The more negative the water potential the smaller the number of pores that need to become hydrophobic to prevent water flow resulting in a lower critical mucilage concentration for lower water potentials. For each water potential there is a certain particle size where soil pores are so big that water cannot percolate even at zero concentration, e.g. $r_{p a r t}=0.01 \mathrm{~cm}$ for $\mathrm{h}=-20 \mathrm{~cm}$.

The capillary rise experiments agree well with the predictions from our model: in the experiment (Fig. 4.6) the percolation threshold for a mucilage concentration of $0.5 \% \mathrm{~g} \mathrm{~g}^{-1}$ and a matric potential of around $-5 \mathrm{~cm}$ is at a range of soil particle sizes of $0.2 \mathrm{~mm}<r_{\text {part }}<$ $0.36 \mathrm{~mm}$. Our model (Fig. 4.7) predicts for a water potential of around $\mathrm{h}=-5 \mathrm{~cm}$ and a soil particle size of around $r_{\text {part }}=0.1 \mathrm{~mm}$ (diameter: $0.2 \mathrm{~mm}$ ) a critical mucilage concentration of $C=0.0025 \mathrm{~g} \mathrm{~cm}^{-3}$ or per unit weight of soil: $C=0.4 \% \mathrm{~g} \mathrm{~g}^{-1}$ which is in the same order as the experimental concentration $\left(0.5 \% \mathrm{~g} \mathrm{~g}^{-1}\right)$.

Mucilage concentration in the rhizosphere depends on several factors: mucilage exudation rate, diffusion of mucilage in the soil (which depends on the soil water content) and root elongation rate. Carminati et al. (in press) calculated the concentration of mucilage for varying rhizosphere extension, assuming exudation rate of $20 \mu \mathrm{g}$ (dry mucilage) per root tip per day and root growth rate of $1 \mathrm{~cm}$ per day. They found that mucilage concentration in the rhizosphere may vary between $0.01-1 \% \mathrm{~g} \mathrm{~g}^{-1}$. It has also to be noted that mucilage concentration varies as a function of distance to the root surface. Our capillary rise experiments were conducted at a mucilage concentration of $0.5 \% \mathrm{~g} \mathrm{~g}^{-1}$, which probably corresponds to the rhizosphere soil very close $(<100 \mu \mathrm{m})$ to the root surface.

In conclusion, we showed that maize rhizosphere turns hydrophobic after drying and subsequent rewetting. We proved that rhizosphere hydrophobicity was caused by mucilage exudation. Mucilage became water repellent after drying. Mucilage contact angle increased with mucilage concentration (gram of dry mucilage per surface area). We developed a simple pore-network model of water percolation through the rhizosphere. The model is capable of reproducing the dual behaviour of the rewetting of soils mixed with root mucilage. When the mucilage concentration is below a given threshold $C$ water can easily cross the rhizosphere. When the mucilage concentration is higher than $C$ the soil becomes water repellent, impeding the water flow.

It has to be mentioned that our model was tested against data derived from a compara- 
bly narrow particle size distribution. The assumed Gaussian distribution of mucilage within the pore space might have been sufficient to match our experimental results, but a preferential distribution of mucilage within the inter-particle space, as observed by Albalasmeh and Ghezzehei (2014), who conducted experiments with polygalacturonic acid (an analogue of mucilage) could be more likely. A preference for high concentrations of mucilage in small pores could also be expected.

A number of assumptions used were clear simplifications of porous media. We assumed a random distribution of mucilage on the soil surface. This assumption may be justified in our experiments, where mucilage was mixed with soil particles of relatively uniform radius. The samples were prepared in a way that mucilage was uniformly distributed - after mixing mucilage and soil, we let the samples dry, we gently crashed them and then we repacked the soil particles. For such an artificial rhizosphere, the assumption of a random mucilage distribution may be justified. However, for a natural rhizosphere, it may well be that mucilage is preferentially distributed in some pore classes. For instance, we can expect that after mucilage is exuded, it is partly drained and it is deposited in the small pores or in the contact between soil particles, as suggested by Albalasmeh and Ghezzehei (2014). This could be modelled by defining a relation between pore size and mucilage concentration and it would have a significant effect on the critical mucilage concentration.

Additionally, in our study we applied a simple site percolation model where all soil particles are represented as spheres of radius $r_{\text {part }}$ arranged in a cubic lattice. A cubic-lattice is a regular structure with a coordination number equal to 6 - each pore site is connected to 6 pore sites. For such a medium, the percolation threshold is $31.2 \%$ (fraction of wettable pores in a site-percolation model). In a real soil, the coordination number is likely to be variable and this would affect the percolation threshold. Therefore, more detailed pore-network models with variable and realistic coordination numbers should be implemented. Such models could be derived from imaging and quantitative morphology of soil pore structure (Vogel and Roth, 2001). Furthermore, corner flow was neglected and particle radii were assumed constant, without implementing a grain size distribution. Finally, our model could be improved by usage of a continuum percolation model, instead of a site percolation model. In this way a random distribution of particles in space and a variable coordination number could be implemented (Balberg, 1987; Berkowitz and Ewing, 1998), without the drawbacks of a regular cubic lattice with a constant coordination number. Further investigations on the pore scale distribution of mucilage in the rhizosphere are necessary to gain a better understanding of 
the role of mucilage on soil-plant water relations. 


\title{
5 Non-equilibrium water dynamics in the rhizosphere: How mucilage affects water flow in soils
}

written by Eva Kröner, Mohsen Zarebanadkouki, Anders Kaestner, Andrea Carminati, published in Water Resources Research (Kroener et al., 2014b)

\begin{abstract}
The flow of water from soil to plant roots is controlled by the properties of the narrow region of soil close to the roots, the rhizosphere. In particular, the hydraulic properties of the rhizosphere are altered by mucilage, a polymeric gel exuded by the roots. In this paper we present experimental results and a conceptual model of water flow in unsaturated soils mixed with mucilage. A central hypothesis of the model is that the different drying/wetting rate of mucilage compared to the bulk soil results in non-equilibrium relations between water content and water potential in the rhizosphere. We coupled this non-equilibrium relation with the Richards equation and obtained a constitutive equation for water flow in soil and mucilage. To test the model assumptions, we measured the water retention curve and the saturated hydraulic conductivity of sandy soil mixed with mucilage from chia seeds. Additionally, we used neutron radiography to image water content in a layer of soil mixed with mucilage during drying and wetting cycles. The radiographs demonstrated the occurrence of non-equilibrium water dynamics in the soil-mucilage mixture. The experiments were simulated by numerically solving the non-equilibrium model. Our study provides conceptual and experimental evidences that mucilage has a strong impact on soil water dynamics. During drying, mucilage maintains a greater soil water content for an extended time, while during irrigation it delays the soil re-wetting. We postulate that mucilage exudation by roots attenuates plant water stress by modulating water content dynamics in the rhizosphere.
\end{abstract}

\subsection{Introduction}

Water uptake by plant roots is a dynamic process that depends on complex interactions between roots and soil. To reasonably include such interactions in root water uptake models is a challenging task, but it is needed to better understand and model how roots take up water from soils.

Current root water uptake models can be grouped into: 1) macroscopic models that describe root water uptake as an empirical sink term, that is proportional to the root length 
distribution and a stress factor (Feddes et al., 1976; Jarvis, 1989; Clausnitzer and Hopmans, 1994); and 2) microscopic models that explicitly solve the flow of water from the soil to root surface (Gardner, 1960). Recent models solve the three-dimensional flow equation in soil and roots (Roose and Fowler, 2004; Doussan et al., 2006; Javaux et al., 2008). In most of these models the soil around roots is considered to be homogeneous with the same hydraulic properties as the bulk soil. Since water flow from soil towards roots is driven by a potential gradient, these models predict that the water content decreases towards the roots.

In contrast to this assumption, there is increasing evidence that the region near the roots, the rhizosphere, has different physical, chemical and biological properties compared to the bulk soil (e.g. Gregory (2006) and Hinsinger et al. (2009)). Young (1995) observed a significantly higher water content in the rhizosphere compared to the bulk soil. Similarly Carminati et al. (2010), Moradi et al. (2011) and Carminati and Vetterlein (2013) (Figure 5.1) showed that even when roots take up water, the water content increased towards the root, which contradicts the assumption that soil is homogeneous around the roots. Some possible reasons for this unexpected increase in water content are the compaction of soil around roots Aravena et al., 2010, 2013) and the hygroscopic properties of mucilage exuded by roots. In this study we focus on the effect of mucilage on root water uptake.

Mucilage is a gel that is capable of holding large volumes of water McCully and Boyer, 1997). Therefore, it is expected that mucilage increases the water content in the rhizosphere at any soil matric potential. On the other hand, mucilage contains also lipids (Read et al., 2003), that may cause a reduction of surface tension of the soil solution. Similarly, lipids could explain the rhizosphere hydrophobicity at lower water contents, as observed by Moradi et al. (2011) and Carminati and Vetterlein (2013). The hydrophobicity for low water contents can be explained by long-chained molecules that change their configuration under dry conditions leading to an exposed hydrophobic surface (Ma'shum and Farmer, 1985).

The physical and chemical principles of gel drying and wetting are discussed in Brinker and Scherer (1990). A gel can be imagined as a liquid inside a solid network. Drying and wetting of gels are driven by osmotic and capillary forces. As the water potential decreases, the solid network of the gel shrinks, and the liquid phase in the gel is drained. As water potential increases, the solid network of the gel swells and the liquid phase is sucked into the gel. The drying and wetting rate depends on gel viscosity, water diffusivity in gel, and on whether the solid network of the gel is elastic, viscous, or viscoelastic.

Drying and wetting of mucilage is a time dependent process and it likely influences water 

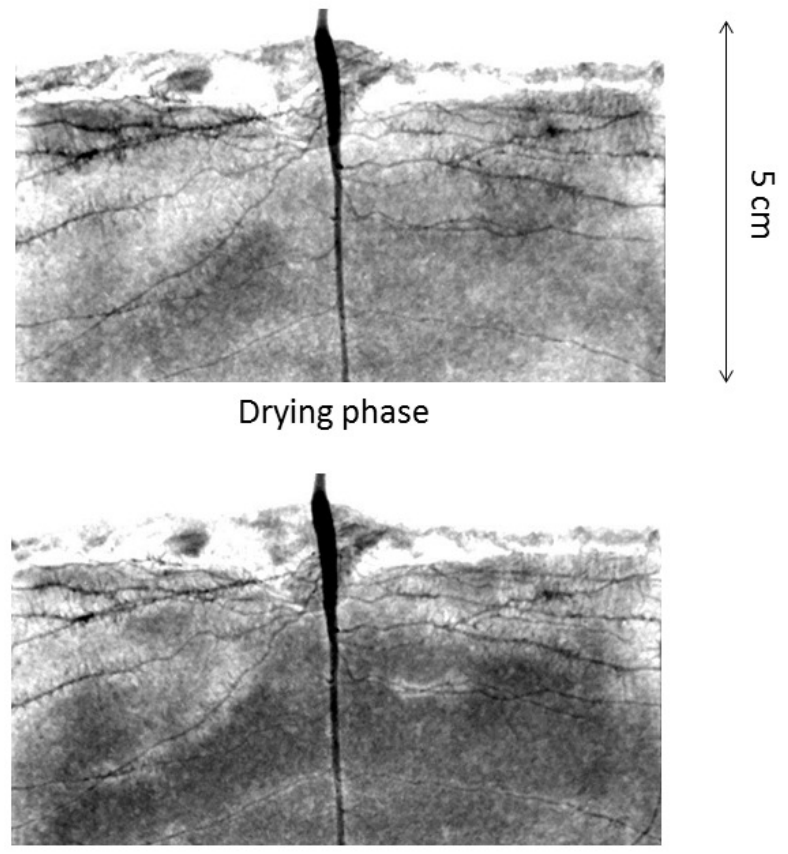

Re-wetting phase

Figure 5.1: Neutron radiography of soil moisture distribution around the roots of lupines. The gray values are proportional to water content (dark means wet). The upper picture was taken during drying, the lower picture was taken 10 hours after irrigation. The radiographs show that the water content in the rhizosphere is markedly different from that in the adjacent bulk soil (adapted from Figure 3 in Carminati and Vetterlein (2013) with kind permission of Oxford University Press). 
content and hydraulic conductivity of the rhizosphere. Therefore, the assumption of a unique relation between water content and matric potential, $\psi(\theta)$, may not be justified in soils mixed with mucilage. On the contrary, this relation may vary over time depending on the drying/wetting history. For example, when mucilage is in equilibrium with the soil water potential, it increases the water content of the rhizosphere at a given water potential. The equilibration time depends, however, on the mucilage re-wetting rate, and as long as the mucilage is not rehydrated it may decrease the water content in the rhizosphere. Such dynamics cannot be correctly described with the classical water retention curve, in which the relation between water content and water potential is unique.

Our objective is to incorporate drying and wetting of mucilage into a model of root water uptake. Depending on the time scale of interest different processes need to be taken into account: exudation, diffusion and degradation of mucilage become important on a time scale of days, drying and wetting of the mucilage happen on a time scale of hours to days (Carminati et al. 2010), while the change in boundary conditions, e.g. during irrigation, may occur on the time scale of a few minutes. In this study we focus on processes that have a time scale of minutes to hours and we do not account for exudation and diffusion of mucilage.

We start from the model of Carminati (2012), in which the re-wetting of the rhizosphere is described with a non-equilibrium term. The model of Carminati (2012) assumed a sharp boundary between bulk soil and rhizosphere. In reality, mucilage concentration decreases from the root surface towards the bulk soil (Li et al., 1997; Gao et al., 2011), and a gradual transition of hydraulic properties from the root surface towards the bulk soil is a more reasonable assumption. This gradual transition is included in the new model by dividing the pore space in two fractions, one filled with mucilage and responsible for the non-equilibrium dynamics, and one not filled with mucilage which follows the classic equations. The model is generalized to allow a non-equilibrium relation between water content and water potential also during the drying phase. In this sense, this model is a more flexible and complete version of the one introduced in Carminati (2012). Finally, the model is now solved numerically, which allows us to simulate variable boundary conditions.

In the initial part of the paper we introduce and provide theoretical justification of the conceptual model. We then describe experiments on water flow in sandy soil mixed with mucilage. We used soil mixed with mucilage extracted from chia seeds as an analogue of the rhizosphere. We then measured the effects of mucilage on the soil water retention curve and the saturated conductivity. Finally we used neutron radiography to image water con- 
tent distribution across the soil-mucilage mixture during drying and wetting experiments. Experimental results have been simulated by numerically solving the model.

\subsection{Material and Methods}

\subsubsection{Model description}

The model is based on the assumption that near the root surface the fraction of pore space filled with mucilage is high. As we move far from the root surface the fraction of pore space filled with mucilage decreases (Figure 5.2 a). We define the non-mucilage-filled pore ratio, $R$, as the ratio between the volume of pores that are not filled with mucilage and the total volume of pores (Figure $5.2 \mathrm{a}, \mathrm{b}$ ). Based on the assumption stated above, we expect that $R$ increases with increasing distance to the root. Similarly, we expect that the non-equilibrium dynamics become less significant far from the root surface (Figure 5.2,, $\mathrm{d}$ ).

In Figure 5.3 we illustrate water and mucilage distribution at the pore scale as a function of distance to the root during a drying cycle. Far from the roots (right side), there is no mucilage and $R=1$. Volumetric water content $\theta$ follows within a very short time the changes in $\psi$. This is expressed as:

$$
\frac{\mathrm{d} \theta}{\mathrm{d} t}=C_{\mathrm{bu}}(\psi) \frac{\mathrm{d} \psi}{\mathrm{d} t}
$$

where $C_{\mathrm{bu}}(\psi)=\frac{\mathrm{d} \theta_{\mathrm{bu}}}{\mathrm{d} \psi}$ is the soil specific water holding capacity, with the subscript bu referring to the bulk soil. This implies a unique function $\theta(\psi)$ for the bulk soil.

When all pores are filled with mucilage, as illustrated in the left column of Figure 5.3 , there is a time delay in the changes in water content. Although the water potential has reached equilibrium, the water content is still changing. Note that this resembles the behaviour of a visco-elastic material. When all pores are influenced by mucilage, the non-equilibrium relation between water content and matric potential is modelled as a relaxation process:

$$
\frac{\mathrm{d} \theta}{\mathrm{d} t}=\frac{1}{\tau}\left[\psi-\psi^{e q}(\theta)\right]
$$

where the equilibrium water potential $\psi^{e q}(\theta)$ is the water potential that the system reaches when kept at water content $\theta$. The parameter $\tau$ can be seen as the relaxation time: the larger it is, the longer it takes for the water content to equilibrate. Eq (5.2) leads to a non-unique relation between water content and matric potential. Such a relation depends on the velocity of the process: when $\psi$ changes slowly, the system is close to equilibrium. Instead, when $\psi$ 
a)

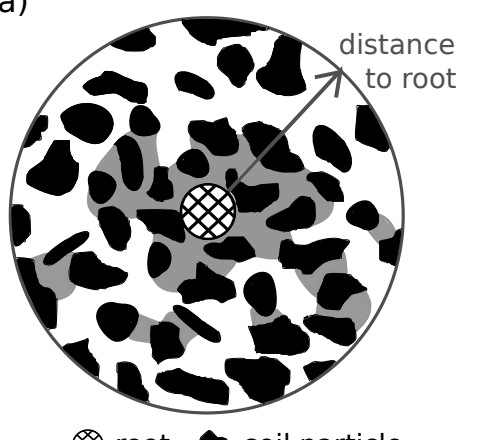

root soil particle

pores influenced by mucilage

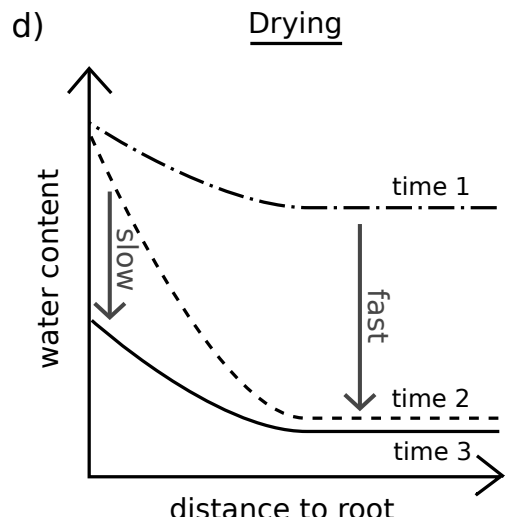

b)

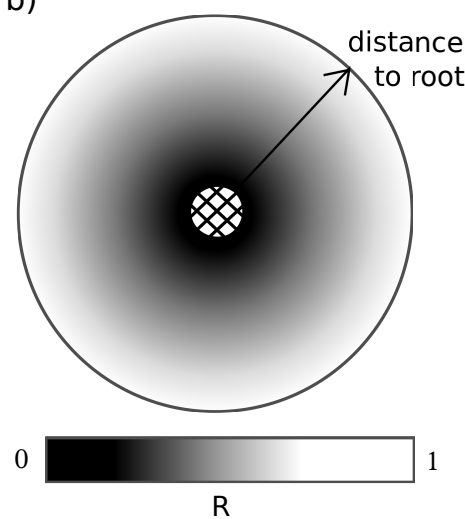

c)

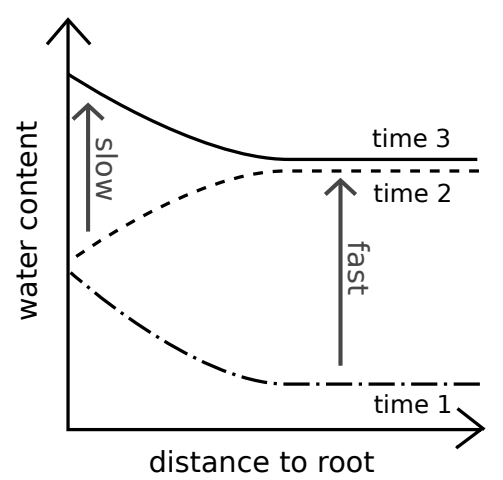

Figure 5.2: Schematic of the physical system simplified into a one dimensional radial geometry. a) Sketch of mucilage distribution in the pore space around a root. b) Radial distribution of non-mucilage-filled pore ratio $R$. c) Dynamic water content change during drying as a function of distance to root. d) Water content change during re-wetting as function of distance to root. 


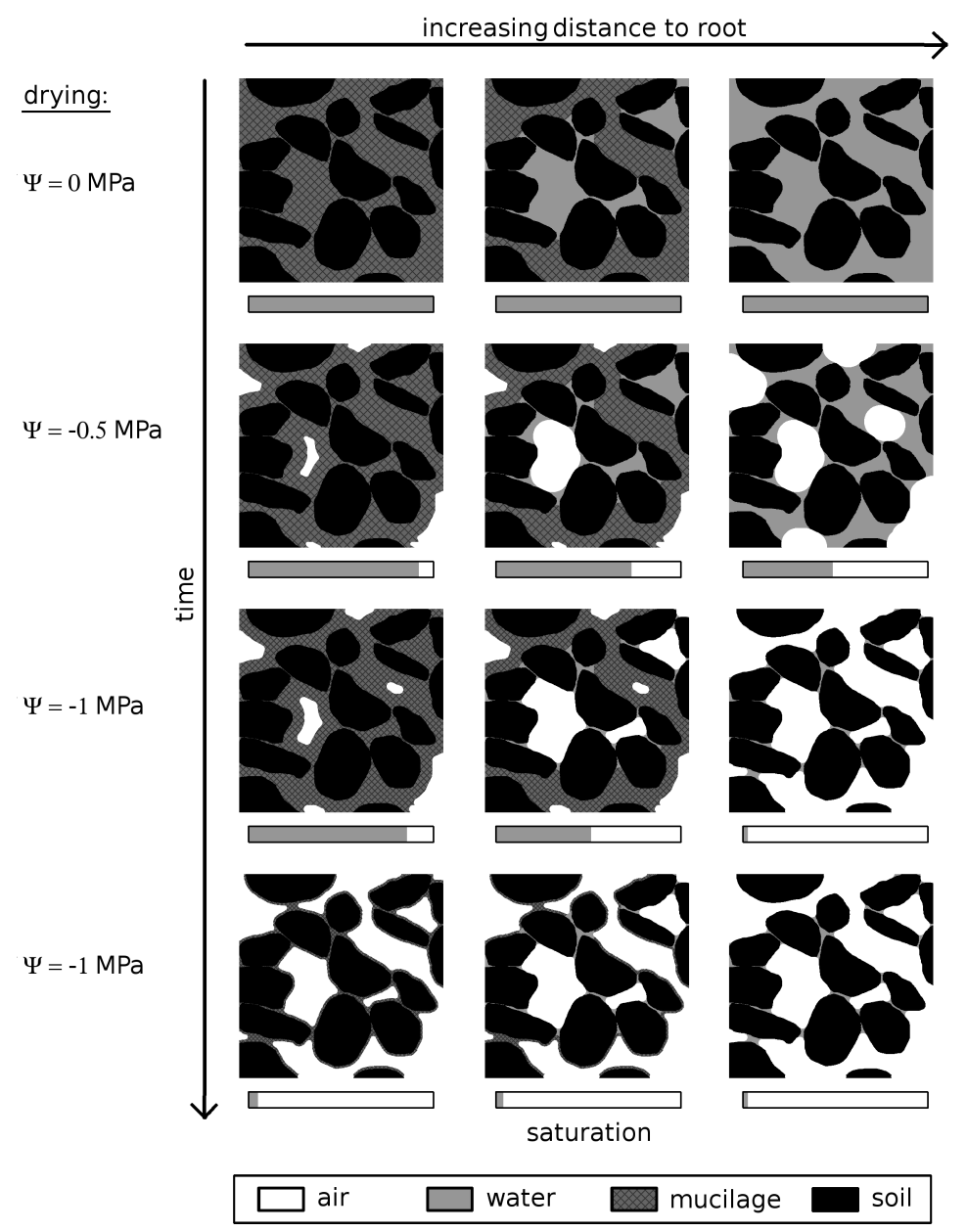

Figure 5.3: Illustration of a drying cycle in the rhizosphere. Near the root the drying process is strongly influenced by the presence of mucilage in the pore space: its gel-like properties induce a delay in water content changes after changes in water potential. Far from the root, where most of the pores are not filled with mucilage, the water content follows the changes in water potential. 


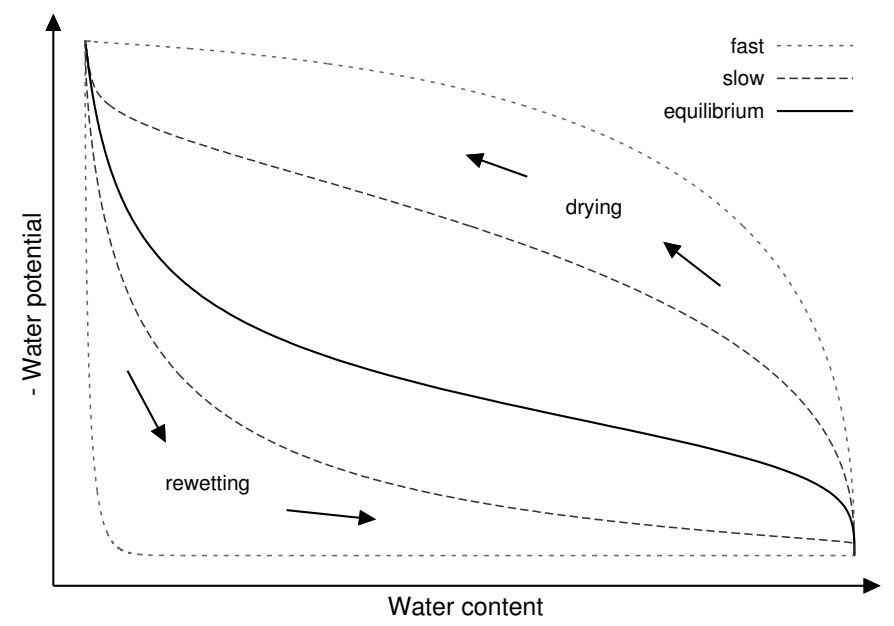

Figure 5.4: Dynamic non-equilibrium in the water retention curve of the rhizosphere. During rewetting/drying cycles we imposed that the water potential increases/decreases exponentially over time. The corresponding water content has been calculated with Eq (5.3). Fast/slow means the applied water potential changes fast/slowly. Here, it is assumed that all pores are filled with mucilage $(R=0)$.

changes rapidly, the time delay in water content change results in a dynamic water retention curve (Figure 5.4).

While Eq (5.1) represents the case of bulk soil $(R=1)$ and Eq (5.2) the situation where all pores are filled with mucilage $(R=0)$, the rhizosphere is in between the two extremes. We assume that the water content in the rhizosphere in an average of the terms in Eq (5.1) and Eq $(5.2)$, with the two terms being weighted by $R$ and $1-R$, with $0 \leq R \leq 1$ :

$$
\frac{\partial \theta}{\partial t}=R C_{\mathrm{bu}}(\psi) \frac{\mathrm{d} \psi}{\partial t}+(1-R) \frac{1}{\tau}\left[\psi-\psi^{e q}\left(\theta_{\mathrm{M}}\right)\right]
$$

where $\theta_{\mathrm{M}}$ is the volumetric water content in the fraction of the elementary volume $(1-R)$ that is influenced by mucilage. The volumetric water content $\theta=R \theta_{\mathrm{bu}}(\psi)+(1-R) \theta_{\mathrm{M}}$ is the sum of the water content $\theta_{\mathrm{bu}}$ in the fraction of the elementary volume that is not influenced by mucilage $R$, and the water content $\theta_{\mathrm{M}}$ of the fraction that is influenced by mucilage $(1-R)$. Note that, assuming uniform soil porosity, the fraction of pore space filled by mucilage, $(1-R)$, is equal to the fraction of the elementary volume, i.e. solid phase + pore space, influenced by mucilage. In other words, if mucilage influences $30 \%$ of the pore space, the fraction of the total volume influenced by mucilage is also $30 \%$. The assumption is that the porosity in the region occupied by mucilage is equal to the porosity in the region without mucilage. The dynamic relation $(\mathrm{Eq} 5.3$ between water potential and water content 

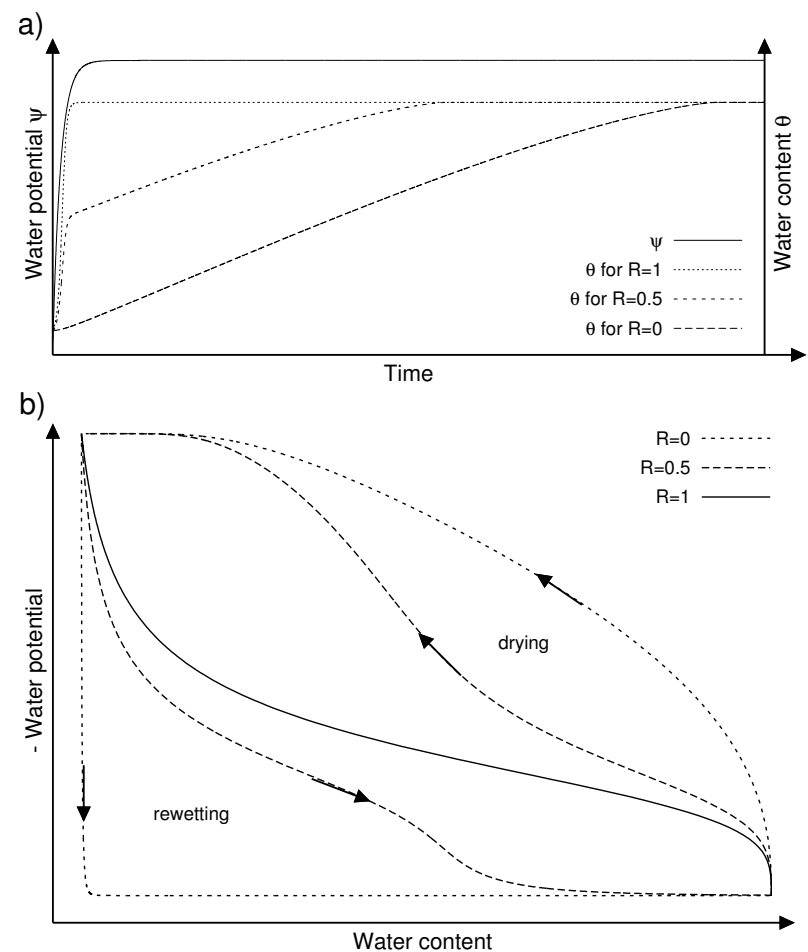

Figure 5.5: a) The water potential increases from a high negative value to a value close to zero. The corresponding water content has been calculated with Eq (5.3): Far from the root $(R=1)$ the change in water content occurs immediately. When mucilage concentration increases $(R=0.5)$ the immediate change in water content occurs in half of the pore fraction, while the remaining half of the pore space reacts with a time delay. At the root surface $(R=0)$ there is no immediate change in water content. In this illustration the possibly different equilibrium water holding capacity of mucilage has not been considered. b) The relation between soil matric potential and water content is illustrated during drying/rewetting (i.e. the applied water potential decreases/increases) for $R=0, R=0.5$ and $R=1$.

is illustrated in Figure 5.5 .

During drying, the time that is needed for mucilage to shrink in response to a decrease in water potential explains the time-delay in the changes in water content. After drying, mucilage turns water repellent (Moradi et al., 2012) and limits the rewetting of the pore space (Fig. 5.6). The increase in water content depends on the mucilage swelling rate, that is controlled by the diffusivity of water molecules in mucilage.

In the following paragraphs the model parameters are discussed in more detail:

Non-mucilage-filled pore ratio The volume fraction, $(1-R)$, of the soil that is influenced by mucilage depends on total mucilage concentration $c_{\text {tot }}$ (dry mass of mucilage per dry mass 


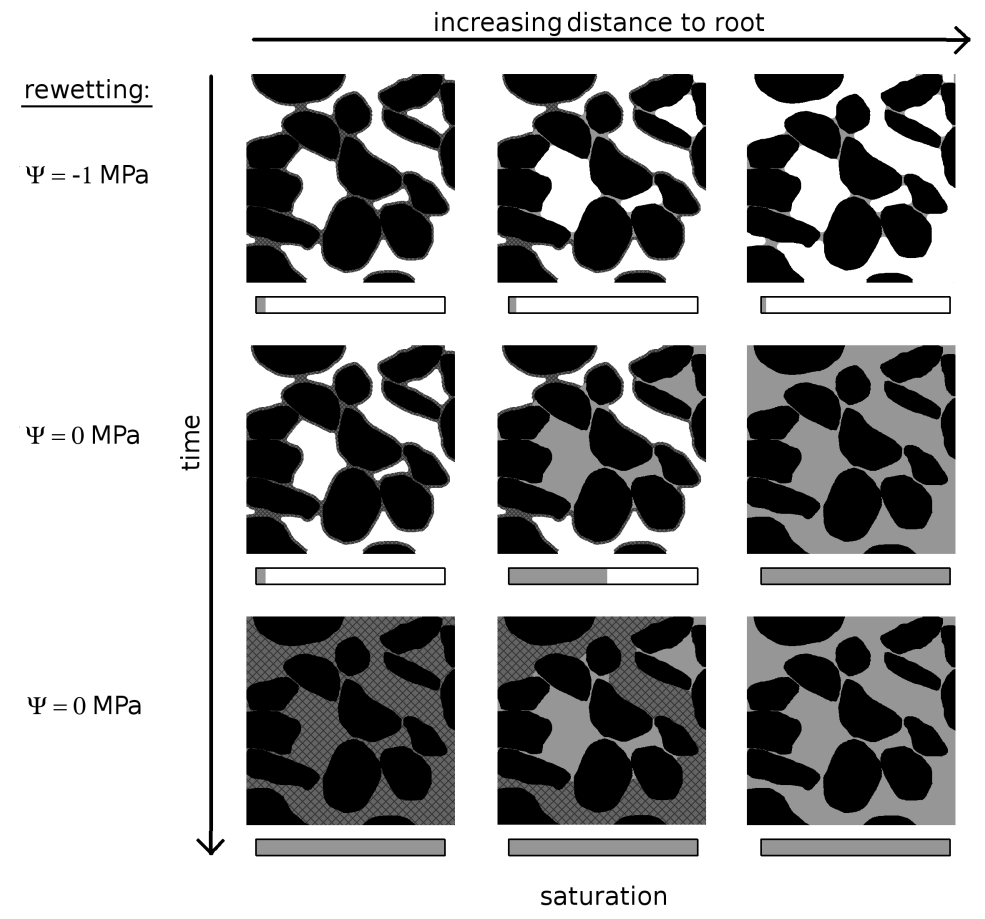

Figure 5.6: Illustration of rhizosphere wetting. Close to the root there is a long time delay in water content change. Far from the root the water content changes at the same time as the water potential. Hydrophobicity of mucilage under dry conditions prevents the immediate re-wetting of the pore space when the surface of surrounding soil particles is covered with gel. 
of soil), soil texture and history of water content. Under saturated conditions the large polymers easily diffuse into soil pores. Additionally, mucilage exudation from roots depends on plant specie, age of the roots and soil water content.

Relaxation time of the rhizosphere The higher is the number and strength of the internal links between mucilage polymers the slower the system equilibrates. At high water contents, the average distance between the polymers increases, while the number of links between them decreases. We therefore expect that the relaxation time $\tau$ is a function of water content in the mucilage-filled pores. In particular, the larger the water content, the faster is the relaxation process. A power function has been chosen to describe this relation:

$$
\tau\left(\theta_{\mathrm{M}}\right)=\theta_{\mathrm{M}}^{-\gamma} \tau_{0}
$$

where $\tau_{0}$ and $\gamma$ are fitting parameters.

Water holding capacity of the rhizosphere Mucilage can hold large volumes of water and in this way it increases the water holding capacity of the soil. In other words mucilage decreases the water potential at a given volume of water. The decrease in water potential is due to osmotic forces. Flory (1953) described a gel as a solution (water plus network of polymers) confined by a membrane. Actually, there is no membrane confining the solute. Instead, the role of the membrane is played by links between the polymers and the development of elastic forces that do not allow the polymers to freely diffuse. Water enters into the gel by osmotic forces and the network expands until water and network are in equilibrium. We assume that the osmotic potential in the gel is related to concentration of mucilage by a power law:

$$
\pi(c)=\omega_{0} c^{\beta}
$$

where $\omega_{0}$ and $\beta$ are fitting parameters. The gravimetric concentration of mucilage in water $c=\frac{c_{\mathrm{tot}} \rho_{\mathrm{bu}}}{\theta_{\mathrm{M}} \rho_{\mathrm{w}}}$ is the ratio of dry weight of mucilage to the weight of water in the mucilage-filled pore space, where $\rho_{\mathrm{bu}}$ and $\rho_{\mathrm{w}}$ are bulk soil density and density of water. For diluted solutions $\beta$ is expected to be 1 (Flory, 1953).

The equilibrium water retention curve as affected by mucilage concentration is related to the water retention curve of bulk soil $\theta_{\mathrm{bu}}(\psi)$ according to:

$$
\theta_{\mathrm{M}}^{e q}(\psi)=\theta_{\mathrm{bu}}\left(\psi+\omega_{0} c^{\beta}\right)
$$




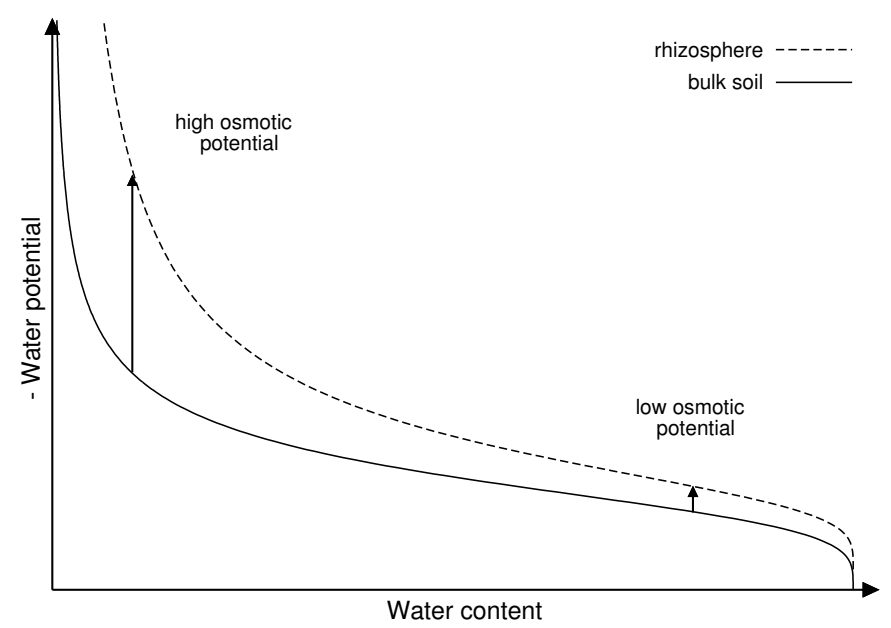

Figure 5.7: Effect of the osmotic pressure in the mucilage on the equilibrium water retention curve of the rhizosphere $(\mathrm{Eq}$ (5.6)). A decrease in water content results in an increase in solute concentration in mucilage and hence in a higher osmotic potential.

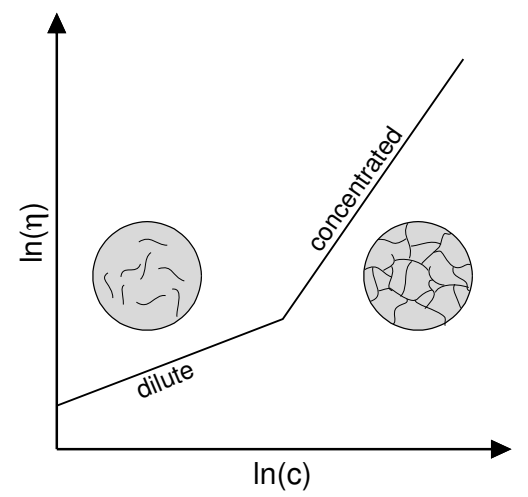

Figure 5.8: Relation between viscosity and concentration of a polymeric solution for dilute and concentrated regime.

Eq (5.6) says that mucilage increases the matric component of the water potential by $\omega_{0} c^{\beta}$. This results in a shift in the water retention curve as shown in Figure 5.7. Eq (5.6) accounts also for the fact that as mucilage dries, its concentration increases, and the osmotic and capillary forces that hold water in mucilage become stronger.

Hydraulic conductivity of the rhizosphere Increasing concentration of mucilage leads to higher viscosity of the liquid phase. Furthermore, the adhesive properties of mucilage lead to links between polymers and soil particles. Both phenomena result in a decrease of hydraulic conductivity of the mucilage-soil composite with increasing concentration of mucilage. 
Morris et al. (1981) investigated the relation between viscosity and solute concentration for polysaccharide solutions. They found two different regimes - one for concentrated solutions and one for dilute solutions. In each of these regimes the viscosity-concentration relation follows a power law with different exponents, as is illustrated in Figure 5.8. The reason of the different exponents is that at a critical concentration the polymers form a network. Above this critical concentration viscosity rapidly increases with increasing concentration.

To account for the mucilage viscosity and additional friction between the polymer network and water, we scale the soil hydraulic conductivity by a factor $\mu(c)$, referred to as relative viscosity, adapted from Morris et al. (1981):

$$
\begin{gathered}
\mu(c)=\max \left\{\begin{array}{l}
{\left[\frac{c}{c_{0}}\right]^{d_{0}}+1} \\
{\left[\frac{c}{c_{1}}\right]^{d_{1}}}
\end{array}\right. \\
K_{\mathrm{eff}}(\theta)=\frac{1}{\mu(c)} K_{\mathrm{bu}}(\theta)
\end{gathered}
$$

where $c_{0}, d_{0}, c_{1}$ and $d_{1}$ are fitting parameters, and $K_{\text {eff }}$ and $K_{\text {bu }}$ are the hydraulic conductivity of soil-mucilage mixture and bulk soil, respectively.

For the conductivity we do not distinguish between mucilage-filled pore space and nonmucilage-filled pore space, and we calculate an effective conductivity by fitting $c_{0}, d_{0}, c_{1}$ and $d_{1}$.

\subsubsection{Numerical solution}

The flow problem was solved in one dimension. Our simulated experiments had a rectangular geometry. Therefore we present the following equations in Cartesian coordinates. For simulating root water uptake they should be reformulated in radial coordinates. The one-dimensional Richard's equation is:

$$
\frac{\partial \theta}{\partial t}=\frac{\partial}{\partial x} K(\theta) \frac{\partial}{\partial x} \psi
$$

By inserting Eq $\sqrt{5.3}$ into Eq 5.9 and considering $C_{\mathrm{bu}} \frac{\partial \psi}{\partial t}=\frac{\partial \theta_{\mathrm{bu}}}{\partial t}$ we obtain:

$$
\begin{aligned}
0 & =R \frac{\partial \theta_{\mathrm{bu}}(\psi)}{\partial t}+(1-R) \frac{1}{\tau}\left[\psi-\psi^{e q}\left(\theta_{\mathrm{M}}\right)\right] \\
& -\frac{\partial}{\partial x} K_{\mathrm{eff}}(\theta) \frac{\partial}{\partial x} \psi
\end{aligned}
$$


Note that if there is no mucilage then $R=1$, and $\mathrm{Eq}(5.10)$ is the classic Richards equation to describe water flow in unsaturated soils.

Gradients in this equation have been expressed by finite differences and Eq (5.10) was solved for $\psi$ with the Newton method. After each iteration $\theta_{\mathrm{M}}$ and $\theta$ have been updated by solving the following implicit equations:

$$
\begin{aligned}
\frac{\partial \theta_{\mathrm{M}}}{\partial t} & =\frac{1}{\tau}\left[\psi-\psi^{e q}\left(\theta_{\mathrm{M}}\right)\right] \\
\frac{\partial \theta}{\partial t} & =R \frac{\partial \theta_{\mathrm{bu}}(\psi)}{\partial t}+(1-R) \frac{\partial \theta_{\mathrm{M}}}{\partial t}
\end{aligned}
$$

The total water content $\theta$, obtained from the above equations, was used to update the conductivity $K_{\text {eff }}$ according to Eq (5.8).

The Thomas-Algorithm has been used to solve the linear equations during Newton iterations. The implementation of the procedure is adapted from (Campbell, 1985), where the approach was described for solving the Richard's equation. Because of the high non-linearity of the problem, an implicit Euler scheme has been chosen for the time discretization.

In each time step the iterations were repeated until: either a maximum number of iterations was reached, or the maximum change in water content, the maximum change in matric potential and the mass balance were smaller than a prescribed tolerance. In the first case the interval of the time-step was divided by two, in the second case the solution was accepted and used for the next time step.

\subsubsection{Parameter estimation and experimental validation of the model}

To validate the model we measured the water retention curve, the hydraulic conductivity and the dynamic drying/wetting behaviour of soil mixed with mucilage. This mixture was created as described below: mucilage was extracted from Chia seeds (Salvia hispanica). Chia seed, lupine and maize mucilage have similar chemical composition (mainly xylose, glucose and uronic acids, (Lin et al., 1994, Carminati and Vetterlein, 2013)) and physical behaviour: when immersed in water seeds release polysaccarides that form a network and build up a transparent, gel-like capsule around the seeds (Lin et al., 1994; Muñoz et al., 2012). When dried the surface of chia seed mucilage turns hydrophobic. This behaviour makes mucilage of chia seeds a good analogue of root mucilage.

Mucilage was extracted in the following way: Chia seeds and water were mixed at a gravimetric ratio of 1:10. After 2 hours, the mixture turned into a gel. We extracted mucilage from the seeds by pushing the gel-like liquid through a sieve. 
We mixed the extracted mucilage with a certain amount of water. The obtained diluted mucilage was mixed with a sandy soil at a ratio of 1:1. In this way, the soil-mucilage mixture was very liquid and could be easily mixed. Throughout our experiments we used a sandy soil (92\% sand, $5 \%$ silt and $3 \%$ clay) collected from the artificial catchment of Hühnerwasser near Cottbus, Germany. It was sieved to a particle size smaller than $1 \mathrm{~mm}$.

The mixture was poured into a wide box and dried for around 24 hours at $60^{\circ} \mathrm{C}$. During drying water evaporates and mucilage deposits in the soil. In this way dry mucilage tends to accumulate on the surface. To reduce this accumulation we used very wide boxes so that the height of the soil was smaller than $1 \mathrm{~mm}$ and mucilage dried homogeneously. The gluey properties of mucilage formed a crust of glued soil particles. This crust was broken into individual soil particles and sieved to a particle size smaller than $1 \mathrm{~mm}$.

Water retention curve We used a pressure plate apparatus to measure the water retention curve of the untreated soil and the soil prepared with mucilage (concentration: $1.25 \%$ dry weight of mucilage per dry weight of soil). Cylinders of $6 \mathrm{~cm}$ in diameter and $6 \mathrm{~cm}$ in height were filled with dry soil. 3 cylinders were filled with untreated soil and 3 with treated soil. The cylinders were saturated by capillary rise. Notably, the capillary rise in the treated soil took approximately 5 days.

The cylinders were placed in the pressure plate apparatus and the water potential was reduced stepwise, with at least two weeks of equilibration time between the steps. At the end of each step the water content was derived from the weight of the sample.

We parameterized the water retention curve of the untreated soil with the Brooks-Corey's model (Brooks and Corey, 1964):

$$
\Theta= \begin{cases}\left(\frac{\psi_{e}}{\psi}\right)^{\lambda} & \text { if }\left(\psi<\psi_{e}\right) \\ 1 & \text { if }\left(\psi \geq \psi_{e}\right)\end{cases}
$$

where $\Theta=\left(\theta_{\mathrm{bu}}-\theta_{\mathrm{r}}\right) /\left(\theta_{\mathrm{s}}-\theta_{\mathrm{r}}\right)$ is saturation, $\theta_{\mathrm{r}}$ residual water content and $\theta_{\mathrm{s}}$ saturated water content. Furthermore $\lambda$ is a fitting parameter and $\psi_{e}$ the air-entry potential.

Saturated Hydraulic conductivity The saturated hydraulic conductivity of soils mixed with mucilage at varying concentration was measured with the falling head method (Klute et al., 2003). We are aware that the falling head measurement of saturated hydraulic conductivity can be affected by flow along the side of the container and macropore flow. We carefully packed the sample and we checked that there were no visible gaps along the inner 
wall of the container.

Hollow aluminium boxes that were open at the top and bottom (width: $1.7 \mathrm{~cm}$, length: $1.7 \mathrm{~cm}$, height: $10 \mathrm{~cm}$ ) were filled to a height of $1.7 \mathrm{~cm}$ with the mucilage-soil mixture. Two meshes made of stainless steel with a mesh size of $0.34 \mathrm{~mm}$ were taped at a height of 0 and 1.7 $\mathrm{cm}$ inside the box to minimize soil displacement during measurement. The soil samples were saturated by capillary rise and left in water for around 50 hours to equilibrate. Then each of them was positioned inside a beaker filled with water. A water filled tube allowed water to flow out of the beaker and in this way kept the water table in the beaker at a constant height of $4 \mathrm{~cm}$. The beaker together with the aluminium box were placed on a balance and the weight was recorded over time.

The aluminium box was initially filled with water. In this way a water potential difference of $6 \mathrm{~cm}$ was applied to the soil column inside the aluminium box leading to a water flow through the soil into the beaker. The additional amount of water left the beaker through the bended tube, leading to a decrease of the recorded weight.

As the change in the difference of water tables over time was proportional to water flow, the decrease of the water in the aluminium box was exponential. The saturated hydraulic conductivity was obtained by fitting an exponential curve to the recorded weight of the sample. We measured the saturated hydraulic conductivity of 30 samples under varying mucilage concentrations.

During each of the measurements we observed that the conductivity did not change significantly even after $100 \mathrm{ml}$ of water had been flowing through the soil sample $(1.7 \times 1.7 \times 1.7$ $\mathrm{cm}^{3}$ ), indicating that the water phase was moving much faster than the gel.

By applying Eq (5.8) the relative viscosity was derived for each of the 30 samples.

Unsaturated hydraulic conductivity In the simulations the Mualem-Brooks-Corey model was used to describe unsaturated hydraulic conductivity of untreated bulk soil:

$$
K_{\text {bu }}(\Theta)=K_{\text {sat }} \Theta^{\frac{2}{\lambda}+2+L}
$$

where $L$ accounts for the tortuosity of the soil. The saturated conductivity $K_{\text {sat }}$ has been measured as described above. Note that knowledge of the saturated conductivity can provide only a very rough estimation of the unsaturated conductivity.

Combining Eq (5.8) and Eq (5.14) provides a value for the unsaturated hydraulic conductivity of the treated soil. 
Mucilage concentration in the water phase (weight of dry mucilage per weight of water $[\mathrm{g} / \mathrm{g}]$ ) was derived from the concentration of mucilage in the soil (weight of dry mucilage per weight of dry soil $[\mathrm{g} / \mathrm{g}])$.

Neutron radiography of drying and wetting experiments Neutron radiography is a non-invasive imaging method that allows to measure spatial distribution of water inside thin samples with high accuracy. We used neutron radiography to monitor water content distribution in soils mixed with mucilage during drying and wetting. The neutron radiography was performed at the cold neutron imaging beam line ICON (Kaestner et al., 2011) of the Paul Scherrer Institute, Switzerland.

The set-up represents a simplified model of the soil-rhizosphere-root system. We used untreated soil to represent the bulk soil (referred to in the results as zone 3), treated soil as rhizosphere (referred to as zone 2) and again a region of untreated soil acting as a root (referred to as zone 1).

The samples were placed in front of the neutron beam. The transmitted intensity of the beam was converted by a scintillator plate into visible light that was acquired by a digital camera and transformed into a digital image. The high neutron attenuation coefficient of water compared to that of air and soil makes neutron radiography an excellent technique to measure water content distribution non-destructively.

For the wetting experiment we placed an aluminium box (width: $1.1 \mathrm{~cm}$, height: $1.5 \mathrm{~cm}$, length: $15 \mathrm{~cm}$; open at the top) horizontally and filled it from the top with dry soil. The first $1.5 \mathrm{~cm}$ was filled with untreated soil (zone 1), the next $0.25 \mathrm{~cm}$ with treated soil (zone 2) and the remaining $13.25 \mathrm{~cm}$ again with untreated soil (zone 3). We injected around $6 \mathrm{ml}$ of water from the far end of zone 3 and we monitored the water distribution with neutron radiography.

To account for a possibly heterogeneous distribution of mucilage in the rhizosphere the treated soil for zone 2 was prepared in the following way: dry untreated soil and dry treated soil prepared with a concentration of $0.43 \%$ weight of dry mucilage per weight of dry soil were loosely mixed at a ratio of 1:2.

For the drying process, we filled an aluminium box (width: $1.7 \mathrm{~cm}$, length: $1.7 \mathrm{~cm}$, height: $10 \mathrm{~cm}$, open at the top, closed only with a nylon membrane at the bottom) with dry soil. The lowest $6.5 \mathrm{~cm}$ was filled with untreated soil (zone 3), the following $0.5 \mathrm{~cm}$ with treated soil (zone 2), and the remaining $3 \mathrm{~cm}$ (zone 1) again with untreated soil. The aluminium box was placed for two days inside a water reservoir to be wetted by capillary rise from the 
Table 5.1: Brooks-Corey parameters fitted to the measured water retention curve of the untreated soil (Eq (5.13)).

\begin{tabular}{cccc}
\hline$\theta_{\mathrm{s}}$ & $\theta_{\mathrm{r}}$ & $\lambda$ & $\psi_{e}[\mathrm{hPa}]$ \\
\hline 0.41 & 0.02 & 0.7 & -13.6 \\
\hline
\end{tabular}

bottom. Afterwards we attached an empty aluminium box of the same size (open at top and bottom) above the prepared aluminium box. We filled the empty aluminium box from the top with dry soil, which applied a suction from the top. The reduction of water content in the lower box was monitored with neutron radiography. For zone 2, we used a soil prepared with a concentration of $0.14 \%$ mucilage.

While the wetting process was monitored in a horizontal set-up, it was convenient to measure the drying process in a vertical set-up. Therefore gravity was included in the simulation of the drying process.

Simulation Initial and boundary conditions were defined according to the experimental set-up. For the wetting process water was injected at positive pressure with a syringe into the soil. We simplified this process in the simulation by assuming that the injected amount of water was homogeneously distributed in the last $11 \mathrm{~cm}$ of the soil sample. Using this water content distribution as initial condition we simulated the spreading of water into the dry part (initial water content: 0.035 ) of the first region of the sample.

Initial conditions for simulating the drying process reproduced the experimental set-up: A wet prepared soil sample at the bottom and a dry soil sample at the top. The initial water content was obtained from the radiographs.

The equilibrium hydraulic parameters of the mucilage-soil mixtures $\left(c_{0}, c_{1}, d_{0}, d_{1}, K_{\text {sat }}, \beta\right.$, $\left.\gamma, \theta_{\mathrm{r}}, \theta_{\mathrm{s}}, \lambda, \psi_{e}, \omega_{0}\right)$ were derived from measured water retention curve and measured saturated hydraulic conductivity. The non-mucilage filled pore space parameter $R$ was defined according to the experimental set-up: for the wetting experiment $R=0.33$ since untreated and treated soil were mixed at a ratio of $1: 2$, and for the drying experiment $R=0$ since treated soil was not mixed with untreated soil. The parameter for unsaturated conductivity $L$ and the non-equilibrium parameters $\tau_{0}$ and $\gamma$ have been fitted to the observed dynamics. 


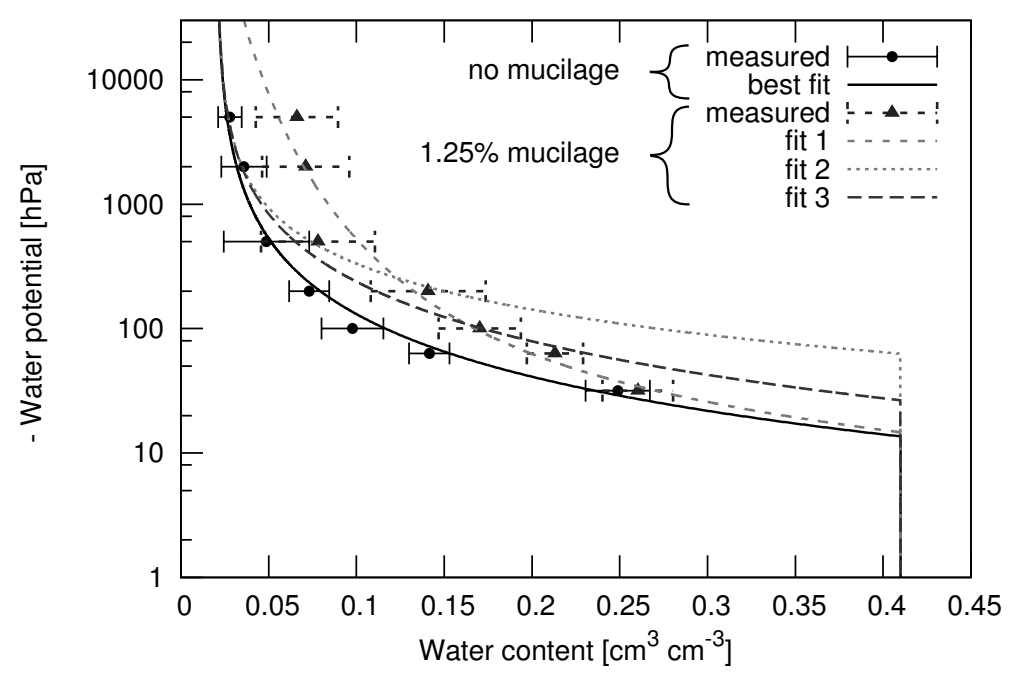

Figure 5.9: Measured and fitted water retention curve for a treated and untreated sandy soil. Measurements as well as fitted lines according to the parameters of Table 5.1 and Table 5.2 are shown.

\subsection{Results}

\subsubsection{Water retention curve}

The water retention curves of treated and untreated soil are presented in Figure 5.9. The experimental data were fitted using Eq (5.13) and Eq (5.6) for untreated and treated soil, respectively. The best fit was obtained with $\beta=4.19$.

According to the osmotic theory, however, the osmotic potential should be proportional to the concentration of the solutes and we would expect a value of $\beta=1$. Our explanation is that there are additional forces between polymers and water molecules that lead to an increase of water holding capacity at low water potentials.

Another explanation is that the unsaturated hydraulic conductivity of the treated soil at low water potentials was so small that the samples could not have reached equilibrium within the two weeks. In this case, one should not rely on the last two points of the water retention curve of treated soil $(\psi<-1000 \mathrm{hPa})$.

As a compromise we calculated the best fit to the values with $\psi>-1000 \mathrm{hPa}$ under the constraint of $\beta=1.5$, see Table 5.2 . This fit was used to simulate drying and wetting process monitored with neutron radiography. 
Table 5.2: Parameters describing the increased water holding capacity caused by mucilage (Eq (5.6)). Fit 1 is the best fit, fit 2 is the best fit under the constraint of $\beta=1$ and fit 3 is the best fit for the measured values with $\psi>-1000 \mathrm{hPa}$ under the constraint of $\beta=1.5$.

\begin{tabular}{cc|cc|cc}
\multicolumn{2}{c|}{ fit 1} & \multicolumn{2}{|c|}{ fit 2} & \multicolumn{2}{c}{ fit 3} \\
\hline$\omega_{0}[\mathrm{hPa}]$ & $\beta$ & $\omega_{0}[\mathrm{hPa}]$ & $\beta$ & $\omega_{0}[\mathrm{hPa}]$ & $\beta$ \\
$1.55 \mathrm{e} 7$ & 4.19 & $2.53 \mathrm{e} 3$ & 1 & $4.75 \mathrm{e} 3$ & 1.5 \\
\hline
\end{tabular}
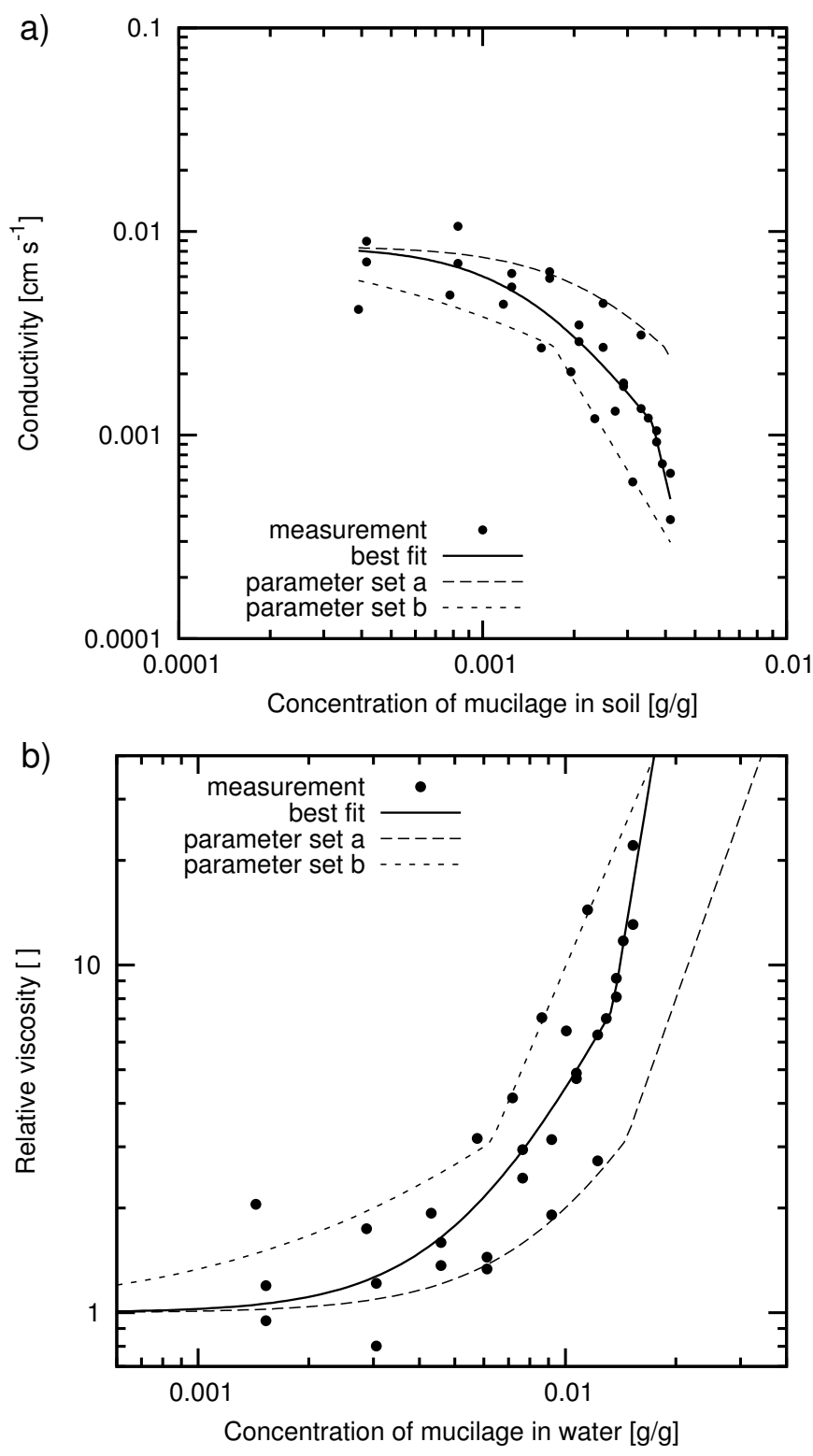

Figure 5.10: a) Saturated hydraulic conductivity as a function of mucilage concentration in a sandy soil. Parameters are listed in Table 5.3 . b) Relative viscosity as function of mucilage concentration in water, derived from soil hydraulic conductivity measurements using Eq (5.8). 
Table 5.3: Parameterization of the reduced relative viscosity (Eq 5.8)). Three parametersets are provided: One for the best fit to the measurements and two modified parameter sets to illustrate the sensitivity towards different parameters.

\begin{tabular}{cccc|ccccc|cccc}
\hline \multicolumn{4}{c|}{ best fit } & \multicolumn{4}{|c|}{ set a } & \multicolumn{5}{c}{ set b } \\
\hline$c_{0}$ & $d_{0}$ & $c_{1}$ & $d_{1}$ & $c_{0}$ & $d_{0}$ & $c_{1}$ & $d_{1}$ & $c_{0}$ & $d_{0}$ & $c_{1}$ & $d_{1}$ \\
0.005 & 1.2 & 0.009 & 8 & 0.01 & 2 & 0.01 & 3 & 0.003 & 1 & 0.004 & 2.5 \\
\hline
\end{tabular}

Table 5.4: Parameters to describe the relaxation process of soil-mucilage mixture (Eq (5.4)). The parameters have been fitted to the drying and wetting process measured with neutron radiography.

\begin{tabular}{cc|cc}
\hline \multicolumn{2}{c|}{ wetting } & \multicolumn{2}{|c}{ drying } \\
\hline$\tau_{0}[\mathrm{~s} \mathrm{hPa}]$ & $\gamma$ & $\tau_{0}[\mathrm{~s} \mathrm{hPa}]$ & $\gamma$ \\
$2.34 \mathrm{e}-4$ & 2 & $1.56 \mathrm{e}-6$ & 2 \\
\hline
\end{tabular}

\subsubsection{Hydraulic conductivity as a function of mucilage concentration}

Figure 5.10 shows the saturated hydraulic conductivity as a function of concentration of mucilage (dry weight of mucilage per dry weight of soil) and the relative viscosity as defined in $\mathrm{Eq}$ (5.8). The relative viscosity is provided as a function of mucilage concentration in water (dry weight of mucilage per weight of water).

The measurements reveal that when concentration of mucilage increases by a factor of 10 , viscosity can increase by a factor of 30 . Figure 5.10 provides two parameter sets (Table 5.3 that envelope the measurements.

\subsubsection{Non-equilibrium monitored with neutron radiography}

Figure 5.11 a shows the water content distribution inside the first $4 \mathrm{~cm}$ of the prepared sample during water infiltration. At time 0 the sample was dry and water was injected from the right side of the sample. After a few minutes zone 1 and 3 rewetted while zone 2 (representing the rhizosphere) remained dry. It shows that water can cross this region without wetting it significantly. While the equilibrium of zone 1 and 3 was reached within a very short time (around $5 \mathrm{~min}$ ), water content in zone 2 continued to increase during the following $30 \mathrm{~min}$. Approximately, 15 min after injection, zone 1, 2 and 3 had similar water content. 35 min after injection zone 2 also appeared to be close to equilibrium. The water contents after 35 min were 0.3 in zone 1 and zone 3 , and 0.32 in zone 2 . This slightly higher water content of 
a)
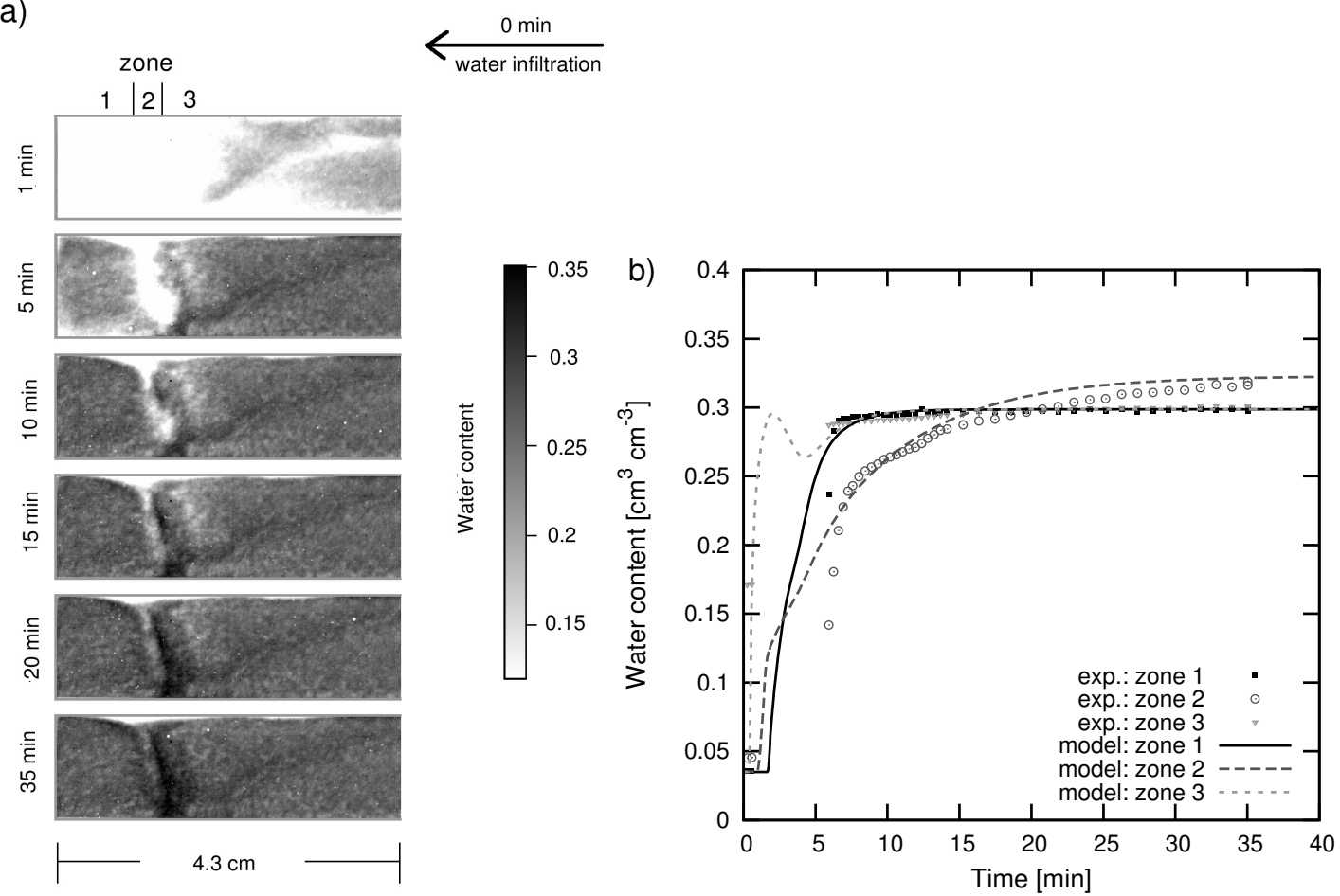

Figure 5.11: a) Water content distribution during wetting as measured with neutron radiography. Zone 1: untreated soil, zone 2: soil mixed with mucilage, zone 3: untreated soil. b) Average water content in the three zones over time. The measured points were obtained from neutron radiographs. The experiment was fitted using the parameters of Table 5.4 . 

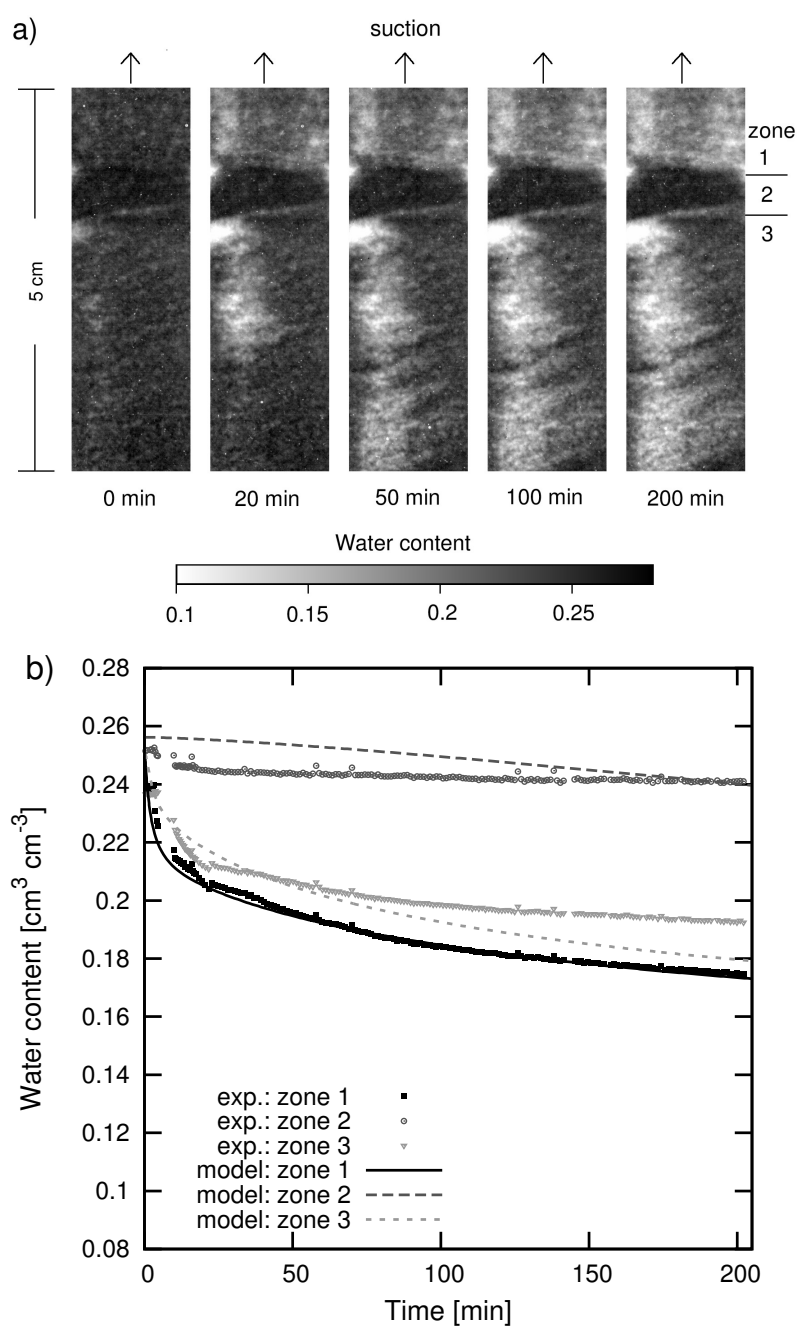

Figure 5.12: a) Water content in soil during drying measured by neutron radiography. Zone 1: untreated soil, zone 2: treated soil, zone 3: untreated soil. b) Water content of the three zones over time. The figure shows measurement and simulation based on parameters of Table 5.4

zone 2 is explained by the different equilibrium water retention curve (Figure 5.9).

Figure 5.11b shows the average water content within the three zones. It is clearly visible that zone 1 and 3 reached their equilibrium within a short time, while it took more than half an hour for zone 2 . In the simulation the relative viscosity was parameterized by the values given as set a in Table 5.3. The results were in good agreement with the measurements.

Figure 5.12 a shows the water content distribution during the drying experiment. The average water contents are plotted in Figure $5.12 \mathrm{~b}$. At time 0 the sample had a water content of around 0.25. Water content in zone 2 was slightly higher than in the bulk soil, which is in good agreement with the water retention curve of treated soil (Figure 5.9). The 
Table 5.5: Mualem-Brooks-Corey parameters of the unsaturated hydraulic conductivity of the untreated soil (Eq (5.14)). $K_{\text {sat }}$ was measured, $L$ was fitted to the wetting and drying experiments observed with neutron radiography.

\begin{tabular}{ccc}
\hline$K_{\text {sat }}\left[\mathrm{cm}^{2} \mathrm{~s}^{-1} \mathrm{hPa}^{-1}\right]$ & $L_{\text {wetting }}$ & $L_{\text {drying }}$ \\
\hline $8.48 \mathrm{e}-3$ & 0 & 2 \\
\hline
\end{tabular}

suction applied by adding dry soil from the top lead to a water uptake into the dry soil (not shown in the image) and as a consequence to a reduction of water content in zone 1 and 3. However, water content in zone 2 stayed almost constant.

Although the water retention of treated soil is higher than that of untreated soil, their slope at $\theta \approx 0.25$ is similar (Figure 5.9p). If the process was always in equilibrium one would have expected a similar drop in zone 2. However, in zone 2 such a sharp drop was not observed. Instead, the water content in zone 2 changed slowly from 0.25 to 0.24 within the first $200 \mathrm{~min}$. According to the simulations this slow change can be understood by assuming a long equilibration time of treated soil during drying. In the simulation the relative viscosity of the soil-mucilage mixture was parameterized by the best fit (Table 5.3 and Figure 5.10).

For the unsaturated conductivity we found good agreement, with $L=0$ in the drying process and $L=2$ in the wetting process (Table 5.5). Their difference might be caused by a different contact between the interfaces of zone 1, 2 and 3 .

Figure 5.13 provides a sensitivity analysis of the drying and wetting process with respect to the parameters $K_{\text {sat }}$ and $\tau_{0}$ : the outcome of the simulations based on the best fit are compared to simulations where either $K_{\text {sat }}$ or $\tau_{0}$ have been multiplied/divided by a factor of 5. Both wetting as well as drying are sensitive to changes in the saturated bulk soil hydraulic conductivity $K_{\text {sat }}$. This is obvious as $K_{\text {sat }}$ affects the parameterisation of all the three zones (Eq (5.8) and Eq (5.14)). However, the relaxation-time parameter $\tau_{0}$ affects only the parametrisation of zone 2 because in zone 1 and zone $3, R$ equals 1 , and the right term of Eq (5.3) vanishes. As a result zone 1 and zone 3 are not very sensitive to $\tau_{0}$ in the drying process. In the wetting process, however, zone 2 remains drier than zone 1 and zone 3 . This means that zone 2 has a very low effective hydraulic conductivity and controls the water flow across the regions during the wetting process. In this way the hydraulic dynamics of zone 1 and zone 3 are strongly affected by the relaxation process of zone 2 when zone 2 is dry. 

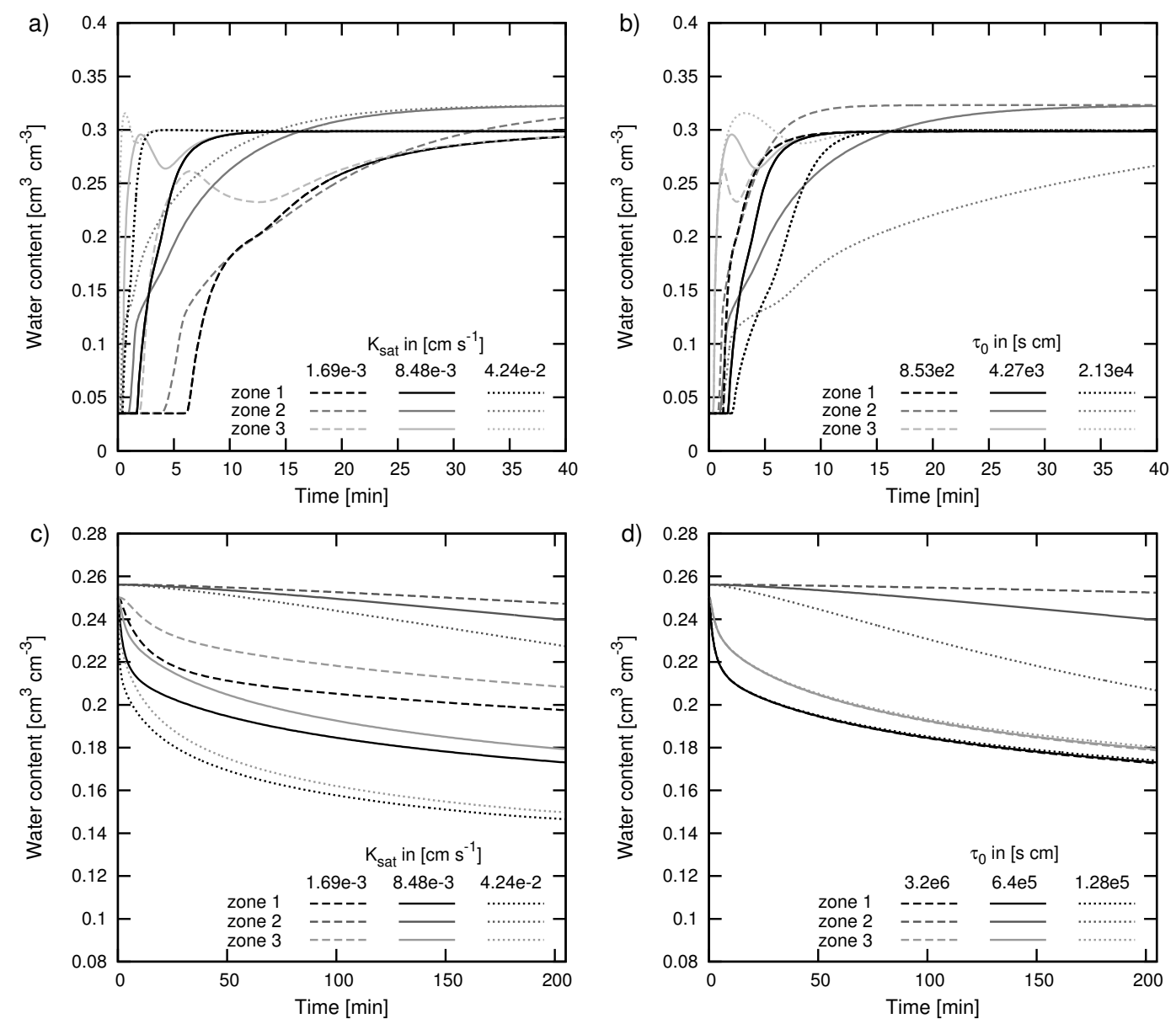

Figure 5.13: Sensitivity analysis of the model to the saturated hydraulic conductivity of untreated soil $K_{\text {sat }}$ and to the relaxation time parameter $\tau_{0}$ during the wetting (a,b) and drying $(\mathrm{c}, \mathrm{d})$ experiments. Continuous lines correspond to the best fit, dotted/dashed lines correspond to simulations where either $K_{\text {sat }}$ or $\tau_{0}$ was multiplied/divided by a factor of five. 


\subsection{Discussion}

We introduced a conceptual model of water flow in soils mixed with mucilage. The model assumes that mucilage alters both the water retention curve as well as the hydraulic conductivity of the soil. Additionally, drying and wetting of mucilage is assumed to result in a non-equilibrium relation between soil water content and matric potential.

The conceptual model was compared to experiments with soil mixed with mucilage extracted from chia seeds. Our measurements showed that:

1. Mucilage increased the soil water content at any given negative matric potential.

2. Mucilage decreased the saturated hydraulic conductivity. The saturated hydraulic conductivity decreased of more than an order of magnitude with increasing mucilage concentration. In particular, the relation between saturated hydraulic conductivity and mucilage concentration was well fitted with a dual-power law.

3. The water content in soil-mucilage mixtures during drying and rewetting showed nonequilibrium dynamics. The non-equilibrium dynamics were well fitted with our model.

The simplified experiments support the main assumption of the model, which consists of introducing a non-equilibrium term in the classic Richards equation.

Our assumption is that the used soil-mucilage mixture is a valid analogue of the rhizosphere. Our choice is partly justified by the observation that the used soil-mucilage mixtures qualitatively behaved as the rhizosphere (Carminati et al., 2010; Moradi et al., 2011; Carminati and Vetterlein, 2013). However, we are well aware that rhizosphere properties vary among plant species, mucilage and root age, bacteria (they produce gel themselves, but also degrade plant mucilage), soil texture, pore size distribution, and history of the water content - i.e. it is hypothesized that plants exude more mucilage in dry conditions. In this sense, our study should not be interpreted as an attempt to parameterize the hydraulic properties of the rhizosphere for including them in root water uptake models. It is more correct to say that the experiments served as a proof of concept of our model.

An additional aspect that we neglected is how far and how quickly mucilage penetrates into the soil upon exudation. Mucilage diffusion in soils certainly depends on water content. In a recent study, Albalasmeh and Ghezzehei (2014) suggested that a certain degree of suction is needed for mucilage to become a gel. Watt et al. (1993) arrived at a similar conclusion. We can expect that mucilage exuded in wet soils will have a low viscosity, will not form a gel 
and it will rapidly spread far from the roots, with a relative loss of its hydraulic benefit in increasing the water content near the roots.

Beside the simplifications in our experimental set-up and modeling approach, we believe that this study is an additional step towards a better understanding of the hydraulics of soil and plants. The hygroscopic properties of mucilage and the consequent high water holding capacity of the rhizosphere is expected to favour water availability to plants during drought. Facilitation in water uptake does not result only from the increased water content of the rhizosphere at equilibrium conditions. The fact that mucilage dries more slowly than the bulk soil - i.e. non-equilibrium dynamics - results in the rhizosphere remaining wetter than what expected at equilibrium. It follows that during periods of increasing transpiration the lowest water content in the rhizosphere is not reached at the transpiration peak, but afterwards. In this way, at the transpiration peak, the rhizosphere is slightly wetter and more conductive compared to the hypothetical case without mucilage.

The slow rewetting of the rhizosphere after drying may locally reduce the uptake of water after a drying/wetting cycle (Zarebanadkouki and Carminati, 2014), but it might also help plants to better tolerate drought. In fact, when the top soil is dry and the sub soil is wet, roots can take up water from the sub soil and the hydrophobic rhizosphere of the upper roots can prevent water from being sucked from the roots into the dry soil.

In conclusion, our study shows how mucilage dynamically affects water flow in unsaturated soils and suggests that mucilage plays a key role in modulating plant water stress during drying and wetting cycles. 
Table 5.6: List of symbols

\begin{tabular}{|c|c|c|}
\hline symbol & description & unit \\
\hline$c_{0}, c_{1}$ & fitting parameters for relative viscosity & {$[-]$} \\
\hline$c$ & gravimetric mucilage concentration in water & {$[\mathrm{g} / \mathrm{g}]$} \\
\hline$c_{\text {tot }}$ & gravimetric mucilage concentration in soil & {$[\mathrm{g} / \mathrm{g}]$} \\
\hline$C_{\mathrm{bu}}$ & water holding capacity of bulk soil & {$\left[\mathrm{hPa}^{-1}\right]$} \\
\hline$d_{0}, d_{1}$ & fitting parameters for relative viscosity & {$[-]$} \\
\hline$K_{\text {eff }}$ & effective hydraulic conductivity & {$\left[\mathrm{cm}^{2} \mathrm{~s}^{-1} \mathrm{hPa}^{-1}\right]$} \\
\hline$K_{\mathrm{bu}}$ & hydraulic conductivity of bulk soil & {$\left[\mathrm{cm}^{2} \mathrm{~s}^{-1} \mathrm{hPa}^{-1}\right]$} \\
\hline$K_{\text {sat }}$ & saturated hydraulic conductivity & {$\left[\mathrm{cm}^{2} \mathrm{~s}^{-1} \mathrm{hPa}^{-1}\right]$} \\
\hline$K$ & hydraulic conductivity & {$\left[\mathrm{cm}^{2} \mathrm{~s}^{-1} \mathrm{hPa}^{-1}\right]$} \\
\hline$L$ & fitting parameters for unsaturated hydr. conductivity & {$[-]$} \\
\hline$R$ & non-mucilage-filled pore ratio & {$[-]$} \\
\hline$t$ & time & {$[\mathrm{s}]$} \\
\hline$x$ & space coordinate & {$[\mathrm{cm}]$} \\
\hline$\beta$ & fitting parameter for osmotic potential & {$[\mathrm{hPa}]$} \\
\hline$\gamma$ & fitting parameter for relaxation time & {$[-]$} \\
\hline$\theta$ & water content & {$\left[\mathrm{cm}^{3} \mathrm{~cm}^{-3}\right]$} \\
\hline$\theta_{\mathrm{r}}, \theta_{\mathrm{s}}$ & residual, saturated water content for water retention curve & {$\left[\mathrm{cm}^{3} \mathrm{~cm}^{-3}\right]$} \\
\hline$\theta_{\mathrm{bu}}$ & bulk soil water content & {$\left[\mathrm{cm}^{3} \mathrm{~cm}^{-3}\right]$} \\
\hline$\theta_{\mathrm{M}}$ & water content in the region influenced by mucilage & {$\left[\mathrm{cm}^{3} \mathrm{~cm}^{-3}\right]$} \\
\hline$\Theta$ & saturation & {$[-]$} \\
\hline$\lambda$ & fitting parameter for water retention curve & {$[-]$} \\
\hline$\mu$ & relative viscosity & {$[-]$} \\
\hline$\pi$ & osmotic potential & {$[\mathrm{hPa}]$} \\
\hline$\rho_{\mathrm{bu}}, \rho_{\mathrm{w}}$ & bulk soil density, density of water & {$\left[\mathrm{g} \mathrm{cm}^{-3}\right]$} \\
\hline$\tau$ & relaxation time & {$[\mathrm{s} \mathrm{hPa}]$} \\
\hline$\tau_{0}$ & fitting parameter for the relaxation time & {$[\mathrm{s} \mathrm{hPa}]$} \\
\hline$\psi$ & water potential & {$[\mathrm{hPa}]$} \\
\hline$\psi_{e}$ & air-entry potential of the water retention curve & {$[\mathrm{hPa}]$} \\
\hline$\psi^{e q}$ & equilibrium water potential & {$[\mathrm{hPa}]$} \\
\hline$\omega_{0}$ & fitting parameter for the osmotic potential & {$[\mathrm{hPa}]$} \\
\hline
\end{tabular}




\title{
6 Mucilage exudation facilitates root water uptake in dry soils
} 6

written by Mutez Ali Ahmed, Eva Kröner, Maire Holz, Mohsen Zarebanadkouki and Andrea Carminati, published in Functional Plant Biology (Ahmed et al., 2015c)

\begin{abstract}
As plant roots take up water and the soil dries, water depletion is expected to occur in the rhizosphere. However, recent experiments showed that the rhizosphere is wetter than the bulk soil during root water uptake. We hypothesize that the increased water content in the rhizosphere is caused by mucilage exuded by roots. It is expectable that the higher water content in the rhizosphere results in higher hydraulic conductivity of the root-soil interface. In this case, mucilage exudation would favor the uptake of water in dry soils. To test this hypothesis we covered a suction cup, referred to as artificial root, with mucilage. We placed it in a soil with a water content of $0.03 \mathrm{~cm}^{3} \mathrm{~cm}^{-3}$, and we used the root pressure probe technique to measure the hydraulic conductivity of the root-soil continuum. The results were compared to measurements with roots not covered with mucilage. The root pressure relaxation curves were fitted with a model of root water uptake including rhizosphere dynamics. The results demonstrated that when mucilage is added to the root surface, it keeps the soil near the roots wet and hydraulically well conductive, facilitating the water flow from dry soils towards the root surface. Mucilage exudation seems an optimal plant trait that favors the capture of water when water is scarce.
\end{abstract}

\subsection{Introduction}

In the coming decades, water is expected to become a major constraint for agriculture worldwide (Steduto et al. 2012). Additionally, precipitation is predicted to become more irregular and sparse, increasing the risks of drought spells and yield loss in large areas of our globe. It is therefore of high interest to better understand the mechanisms that help plants to better tolerate drought.

According to Blum (2005), plants can resist to drought either by dehydration avoidance or by dehydration tolerance. To limit the risk of dehydration, plants can reduce transpiration by

\footnotetext{
${ }^{6}$ E.K. was responsible for the modeling part and was partly involved in the experimental design of the study.
} 
closing stomata or they can grow roots that are more efficient in extracting water from soils, in particular as the soil water content decreases. This result can be achieved, for instance, by plants with deep roots and shallow growth angles (Lynch, 2013). Such a root trait should allow crops to more easily extract water stored in the subsoil. Besides altering themselves, plant may also change their surroundings properties to optimize the access to water. Here, we propose another plant trait that is expected to increase the ability of roots to extract water from dry soils: mucilage exudation.

Mucilage is a polymeric gel that is exuded by most of plant roots. It is primarily composed of polysaccharides, but it contains also a small fraction of lipids (Read et al., 2003). Notably, fully hydrated mucilage has a water content of $105 \%$ (percentage of dry weight). McCully and Boyer (1997) discussed the potential role of mucilage and its large water holding capacity in protecting roots from drought. They found that mucilage loses most of its water at water potentials higher than -0.01 MPa and concluded that mucilage water content, per-se, does not play a significant role on drought protection. Indeed, the volume of water hold by mucilage when the soil is dry would be quickly consumed by plants.

To better understand the role of mucilage in plant-soil water relations it is useful to look at the fluxes of water, rather than only at the volumes of water. Carminati et al. (2011) calculated the gradients in water potential in the soil near roots covered and not with mucilage. In this modeling study, they hypothesized that: 1) mucilage increases the water content in the rhizosphere at any water potentials; 2) mucilage increases the viscosity of the soil solution and, consequently, it decreases the saturated conductivity of the rhizosphere; and 3) thanks to the increased water content, the rhizsophere has a higher unsaturated conductivity at low water potentials. The simulations showed that mucilage facilitated the water uptake of roots. Thanks to mucilage, roots had a higher water potential during the drying period, wilted at a lower soil water potential and wilted later. Carminati and Vetterlein (2013) elaborated further on this result and suggested that rhizosphere plays a key role in the acquisition of scares resources. However, the role of mucilage and rhizosphere on root water uptake and drought tolerance has not yet been demonstrated and remains largely hypothetical.

In this manuscript we aimed to experimentally test the hypothesis that mucilage exudation facilitates root water uptake from relatively dry soils. Since measuring water flow in soil-root systems is not trivial and the overall hydraulic conductance of the root-soil system depend on the properties of the roots, rhizosphere and soil, which can all be dynamic and hysteretic, we 
simplified the problem as it follows: we substituted real roots with artificial ones - namely, we used suction cups of known hydraulic conductivity, and we used mucilage extracted from chia seeds (Salvia Hispanica L.). The reasons of using chia seeds are two. The first one is practical: chia-seeds produce large quantity of mucilage. The second one is that the chemical composition of mucilage of chia seeds is similar to that of maize: both are mainly composed of xylose, glucose, and uronic acids (25\%). Furthermore, mucilage from chia seeds has similar physical characteristics to maize and lupine mucilage: it forms a gel when it is hydrated and it turns hydrophobic after drying.

The water flow into artificial roots covered with mucilage and placed into a sandy soil was measured with the root pressure probe technique (Liu et al., 2009). We employed the pressure probe to monitor the pressure relaxation after imposing a given flow of water into or out of artificial roots covered and not with mucilage. The root pressure probe has been typically used to measure the hydraulic conductivity of excised roots in hydroponics. Here, we extended the technique to roots in relatively dry soils. The non-linearity of the soil hydraulic conductivity as a function of the soil water content makes the interpretation of the relaxation experiment not trivial. Therefore, we developed a numerical model that simulates the water flow across soils mixed with mucilage. The novelty of the model consists in adding a non-equilibrium term to the Richards' equation that is usually used to simulate water flow in soils. The method is an extension of the non-equilibrium model of Carminati (2012).

We used this method to determine the hydraulic conductivity of the soil near the root when mucilage was present or not. We aimed to test the hypotheses that mucilage facilitates the water flow into the roots and it increases the soil hydraulic conductivity. In a complementary experiment, we tested to what extent mucilage is able to hold water when injected into a relatively dry soil.

\subsection{Materials and methods}

Mucilage preparation We used mucilage from Chia seeds (Salvia hispanica L.) as a model of plant mucilage. The chemical composition of mucilage of chia seeds is similar to that of maize: both are mainly composed of xylose, glucose and uronic acids. In both mucilages, the content of uronic acid is around 25\% (Lin et al., 1994; Carminati and Vetterlein, 2013). Furthermore, mucilage of chia seeds showed a physical behavior similar to that of mucilage from maize and lupines. It has been shown that mucilage of chia seeds contains significant amounts of polysaccharides that form a gel-like network around the seeds (Lin et al., 1994; 
Muñoz et al., 2012). Indeed, when we immersed chia seeds in water, mucilage appeared immediately as a transparent capsule around the seeds. We also dried the mucilage of chia seeds and observed a high contact angle and consequent hydrophobicity.

Mucilage was extracted with the following procedure: we added $50 \mathrm{~g}$ of distilled water to $5 \mathrm{~g}$ of chia seeds. The mixture was stirred with a magnetic stirrer for 2 minutes and then kept for 2 hours at room temperature. The mixture was then pushed through a sieve using a syringe that was cut at the end. In this way, we separated the seeds from their mucilage. It has to be mentioned that part of the mucilage, the most gluey and gel-like, remained attached to the seeds.

Soil preparation We used a sandy soil collected from the artificial catchment of Chicken Creek located near Cottbus, Germany. The soil (sieved to a particle size smaller than $1 \mathrm{~mm}$ ) consisted of $92 \%$ sand, $5 \%$ silt and $3 \%$ clay. We packed the soil homogeneously into aluminum containers of size $6 \mathrm{~cm} \times 10 \mathrm{~cm} \times 1.5 \mathrm{~cm}$. For the soil packing, we poured the soil through a sieve while the containers were laid horizontally to minimize soil layering. This procedure resulted in a soil with a bulk density of $1.45 \mathrm{~g} \mathrm{~cm}^{-3}$. Then, we put the samples on a balance and we slowly added $2.5 \mathrm{~g}$ of water from the top into the soil. This resulted in an average soil water content of $0.03 \mathrm{~cm}^{3} \mathrm{~cm}^{-3}$.

Water retention curve and saturated hydraulic conductivity We determined the water retention curve of the soil mixed with and without mucilage using a pressure plate apparatus. We filled three cylinders of $6 \mathrm{~cm}$ diameter and $6 \mathrm{~cm}$ height with dry soil. We slightly compacted the soil to achieve a density of $1.45 \mathrm{~g} \mathrm{~cm}^{-3}$ and we saturated it with water. We placed the cylinders in a pressure plate apparatus and determined the soil water content at different water potential. In order to determine the retention curve of the soil mixed with mucilage, we first mixed the mucilage with the soil. Mucilage was extracted from the chia seeds as described above. After extraction, mucilage was well hydrated and had a concentration of $0.0125 \mathrm{~g} \mathrm{~g}^{-1}$ (g of dry mucilage per $\mathrm{g}$ of water). We mixed $600 \mathrm{~g}$ of soil with $600 \mathrm{~g}$ of wet mucilage. Then, we incubated the mixture in the oven at $60^{\circ} \mathrm{C}$ for 24 hours. Three cylinders were filled with the treated soil and the water content was measured at different water potential in the pressure plate apparatus. Note that to wet these samples, we had to leave them immersed in water for 5 days. We also measured the saturated hydraulic conductivity of the soil without mucilage by applying a constant pressure difference at the top and bottom of the cylinders and measuring the outflow. 
Drying rate of mucilage in soil The objective of this experiment was to estimate how long and to what extent mucilage maintains the soil near the roots wet. We filled four containers of $9.5 \mathrm{~cm} \times 9 \mathrm{~cm} \times 8 \mathrm{~cm}$ with $500 \mathrm{~g}$ of dry soil. The packing resulted in a bulk density of $1.45 \mathrm{~g} \mathrm{~cm}^{-3}$ and a soil depth of $4 \mathrm{~cm}$. Then, we added $14 \mathrm{~g}$ of water to the soil to reach a water content of $0.04 \mathrm{~cm}^{3} \mathrm{~cm}^{-3}$. We closed the lid of the plastic box tightly and let the soil water content equilibrate for 10 hours. Then, we injected $0.20 \mathrm{~g}$ of mucilage in 7 points at regular intervals of ca. $3 \mathrm{~cm}$.

After injection, we closed the containers with a plastic lid to avoid evaporation. Then, we collected soil samples at different times: $0,3,5,7,9,12$ and 24 hours after mucilage injection. The soil samples were collected using an auger with a diameter of $0.4 \mathrm{~cm}$ and a length of $7 \mathrm{~cm}$. This experiment aimed to determine the water content of the soil regions embedded with mucilage over time. As a control experiment, we repeated the measurements injecting water (same amount), instead of mucilage. We repeated these experiments in four replications.

Pressure probe experiment We used a root pressure probe technique to quantify the easiness of the water flow into artificial roots covered and not with mucilage and placed into the soil. The technique has been widely used to measure the hydraulic conductivity of roots in hydroponics (Frensch and Steudle, 1989: Ranathunge et al., 2004, Knipfer and Fricke, 2010). Here, we applied the technique to artificial roots sitting in a relatively dry soil.

The root pressure probe consists of an oil-filled pressure chamber attached to a pressure transducer. The transducer converts the pressure to a voltage recorded on a computer. A metal rode is sealed inside the pressure chamber. The metal rode can be turned forward (or backward) to impose a flow out (or into) the artificial root. The two directions induce an injection or suction, respectively. A glass capillary with an internal diameter of $300 \mu \mathrm{m}$ was attached to the pressure chamber and connected to the artificial root with the aid of a silicon robber seals (Fig. 6.1). The first half of the capillary glass attached to the pressure chamber was filled with silicon oil and the half connected to the artificial roots was filled with degassed-water. A meniscus formed at the oil-water interface that allowed us to control the volume of water moved during the injection and suction experiments.

We used an artificial root as a model system to mimic a plant root. This choice let us focus on the effects of mucilage, without having the complexity of a real root with its possibly varying hydraulic conductivity. The artificial root consisted of a hollow cylinder made of a nylon membrane, a metal rod in the middle of the cylinder for mechanical support, and a 


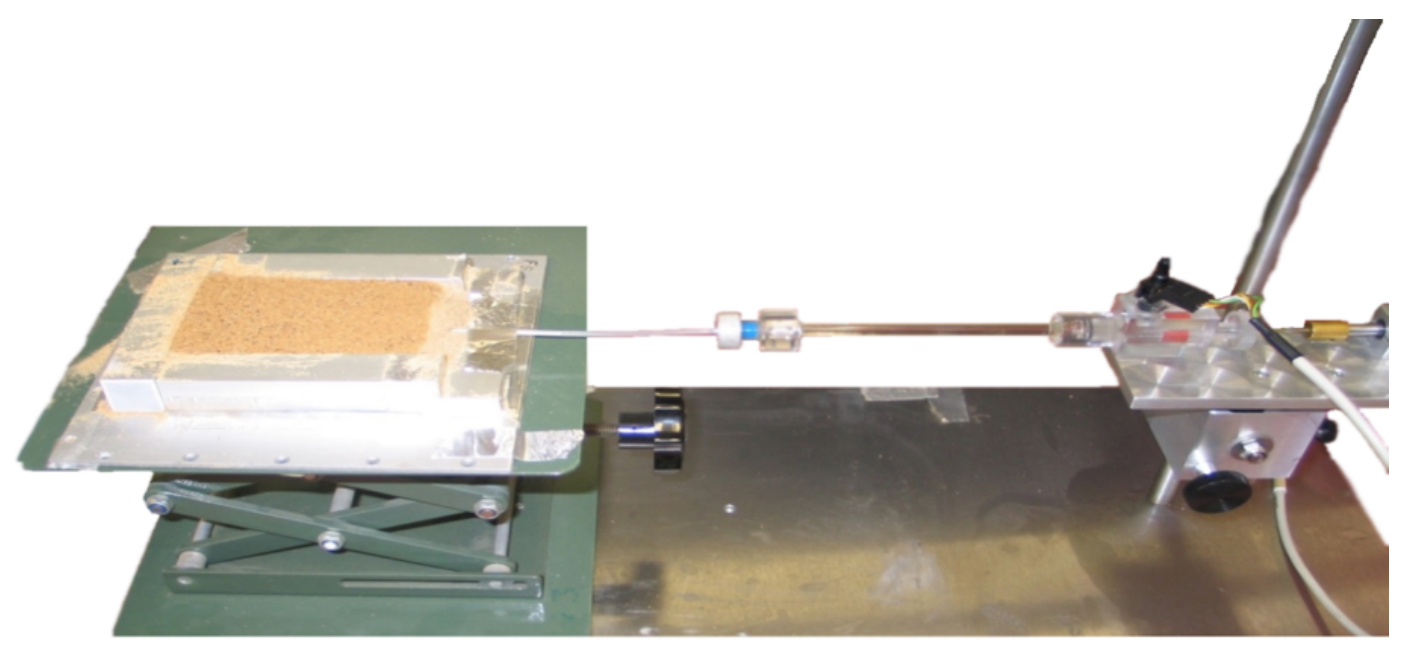

Figure 6.1: The experimental set-up for the root pressure probe experiment.

teflon tube that was glued to the nylon hollow cylinder. The permeating part of the root was $0.2 \mathrm{~cm}$ in diameter and $4.5 \mathrm{~cm}$ in length.

The soil had initially an average water content of $0.03 \mathrm{~cm}^{3} \mathrm{~cm}^{-3}$. A slight depression was formed on the soil surface and the artificial root was placed inside. We uniformly covered the artificial root with $0.5 \mathrm{~g}$ of wet mucilage. We added $0.4 \mathrm{~cm}$ of dry soil on the artificial root and we gently pressed the soil to ensure a good contact of the soil with the artificial root. Then, we covered the top of the soil with a plastic foil to minimize evaporation from the soil. Evaporation was nevertheless monitored during the experiment by leaving the sample on the balance throughout the measurement period. We waited for 1 to 2 hours until the pressure measured by the root pressure probe was constant. We refer to the measured pressure as the root pressure. Then, we induced a given volume of water into or out of the artificial root by turning the metal rode and we monitored the root pressure relaxation. We applied several injection and suction pulses at different times for a period of 24 hours. At the end of the experiment, we removed the artificial root from the soil and placed it in water to measure the half time of its relaxation. We compared the half time of the pressure relaxation in water at the end of the experiment with the one at the beginning to test if cavitation occurred during the measurements. We repeated this experiment in three replications.

As a control, we repeated the pressure probe experiment in the same set-up with the only difference that, instead of $0.5 \mathrm{~g}$ mucilage, we added $0.5 \mathrm{~g}$ of water on the top of the root. 
We applied several suction and injection pulses at different times for a period of 6 hours. We conducted this experiment in three replications.

The hydraulic conductivity of the artificial root was measured by immersing the artificial roots in water and applying a given suction or injection pulse. Determination of the hydraulic conductivity of the artificial root sitting in a nutrient solution is straightforward In this case, the pressure relaxation follows an exponential curve and the half time is inversely proportional to the hydraulic conductance of the system (Steudle et al., 1987).

The pressure relaxation curve in roots in soils is not exponential. Due to the movement of water through the soil and the non-linearity of the soil hydraulic properties, interpretations of the relaxation time requires the simulation of water flow across roots and soil, with the additional complexity of mucilage dynamics. The model description is explained in the following section.

Model of water flow in roots and soil including mucilage dynamics The experiments were simulated solving Richards' equation of water flow in soils. We used two models for the soil hydraulic properties, depending on the quantity of mucilage in the soil: if no mucilage was present in the soil, we assumed a unique function relating the volumetric water content $\theta\left[\mathrm{cm}^{3} \mathrm{~cm}^{-3}\right]$ to the soil matric potential $h$, here expressed in centimeter heads $[\mathrm{cm}]$; this relation is called soil water retention curve $\theta(h)$.

When mucilage was present in the soil, we assumed that the relation between $\theta$ and $h$ is not unique, but it changes over time. Carminati (2012) modeled the dynamic relation between $\theta$ and $h$ introducing a non-equilibrium term in the Richards' equation. Here, we further developed this concept to enable a continuous transition of hydraulic properties from the root surface (where the concentration of mucilage is high) to the parts that are more distant from the root (where there is no mucilage).

The idea behind the non-equilibrium between $\theta$ and $h$ is that the gel-like properties of mucilage cause a time delay in the water content changes. Since a certain fraction of the pore volume $R$ may not be influenced by mucilage - e.g. because mucilage does not diffuse homogeneously in all the pores, the model of Carminati (2012) is modified as:

$$
\frac{\partial \theta}{\partial t}=R C_{\text {soil }}(h) \frac{\partial h}{\partial t}+(1-R) \frac{1}{\tau}\left[h-h^{e q}\left(\theta_{G}\right)\right]
$$

where $C_{\text {soil }}=\frac{d \theta_{\text {soil }}(h)}{d h}$ is the water holding capacity of the bulk soil, $R$ is the fraction of the pore volume that is not influenced by mucilage - e.g. because mucilage does not diffuse homogeneously in all pores, $\tau$ is related to the relaxation time of the non-equilibrium process, 
$\theta_{G}$ is the water content in the mucilage-filled pore space, and $h^{e q}\left(\theta_{G}\right)$ is a function that relates the matric potential that is reached if the mucilage-filled pore space is kept at water content $\theta_{G} \cdot$

The parameter $R$ in Eqn. (6.1) can be seen as a weighting factor between the water holding properties of the pores that are not filled with mucilage (first term of the right side) and the non-equilibrium water holding dynamics of the mucilage-filled pores (second term of the right side). The parameter $\tau$ can be considered as the relaxation time of the process: A small $\tau$ (short relaxation time) leads to a large value of the second term resulting in a fast change in water content and in a fast equilibration time.

In our simulation the weighting factor $R$ depends on the concentration of mucilage $C_{t o t}$ (weight of dry mucilage per weight of dry soil):

$$
R\left(C_{t o t}\right)=e^{-a C_{t o t}}
$$

where $a$ is a fitting parameter that depends on soil and mucilage properties. Eqn. 6.2 guarantees that the hydraulic properties and non-equilibrium dynamics are not much affected when mucilage concentration is low. Due to diffusion of mucilage into the soil, it is reasonable to assume that the concentration of mucilage decreases continuously from a certain value at the root surface to zero at a certain distance from the root surface in the soil.

The relaxation time depends on the water content in the gel-filled pores:

$$
\tau\left(\theta_{G}\right)=\theta_{G}^{-\gamma} \tau_{0}
$$

where $\gamma$ and $\tau_{0}$ are the fitting parameters. Eqn. (6.3) guarantees that the relaxation time is faster when soil and mucilage are wet.

Water holding capacity The water retention curve of the soil was fitted with the BrooksCorey parameterization (Brooks and Corey, 1964).

$$
\Theta=\left(\frac{h}{h_{0}}\right)^{-\lambda}
$$

where $\Theta=\left(\theta-\theta_{r}\right) /\left(\theta_{s}-\theta_{r}\right)$ is the saturation, $\mathrm{h}_{0}[\mathrm{~cm}]$ is the air-entry value and $\lambda$ is a fitting parameter.

Mucilage is expected to increase the soil water content at negative water potentials. Flory (1953) described the mucilage as a concentrated solution enclosed in a membrane. Actually, the membrane does not exist, but is introduced to represent the forces between the polymers that do not let the polymers diffuse away. The resulting osmotic forces depend on the 
concentration of mucilage in the water phase. The following equation has been used to fit the measured water retention of the mucilage samples:

$$
h_{\text {gel }}(\theta)=h_{\text {soil }}(\theta)-\omega_{0}\left(\frac{C_{t o t}}{\theta}\right)^{\beta}
$$

where $\omega_{0}$ and $\beta$ are the fitting parameters and $h_{\text {soil }}(\theta)$ is the inverse of the water retention curve of the soil. The right term takes into account additional forces caused by the presence of mucilage. Theoretically, the exponent $\beta$ should equal 1. This estimation is based on the gas theory. Mucilage, however, consists of long polymers, that form a network with bonds that likely vary with water content. Therefore, we considered $\beta$ as a fitting parameter.

Hydraulic conductivity The Mualem-Brooks-Corey model was used to describe the unsaturated hydraulic conductivity:

$$
K(\Theta)=K_{\text {sat }} \Theta^{\frac{2}{\lambda}+2+L}
$$

where $L$ is the tortuosity.

The parameter $L$ was estimated by fitting the root pressure relaxation in the experiments without mucilage.

The presence of mucilage in the liquid phase is expected to increase the viscosity of the soil solution. The increase of viscosity due to solutes is described with the following law modified from Morris et al. (1981).

$$
\mu(c)=\mu_{\omega}\left(1+v c^{d}\right)
$$

where $c$ is the concentration of mucilage in the liquid face, $\mu_{\omega}$ is the viscosity of water, $v$ and $d$ are the fitting parameters.

The viscosity of the liquid phase leads to the following reduction in hydraulic conductivity:

$$
K_{G}(\Theta)=\frac{\mu_{\omega}}{\mu(c)} K(\Theta)
$$

Numerical solution The experiments were simulated in a one-dimensional radial geometry. The Richards' equation was solved according to Campbell (1985): the mass balance for each note was minimized with a Newton-Raphson method, where in each step the ThomasAlgorithm was used to solve the linearized equations.

Due to the high non-linearity of the problem, an implicit Euler scheme was chosen for the time discretization. Because of the non-equilibrium dynamics, water potential and water 


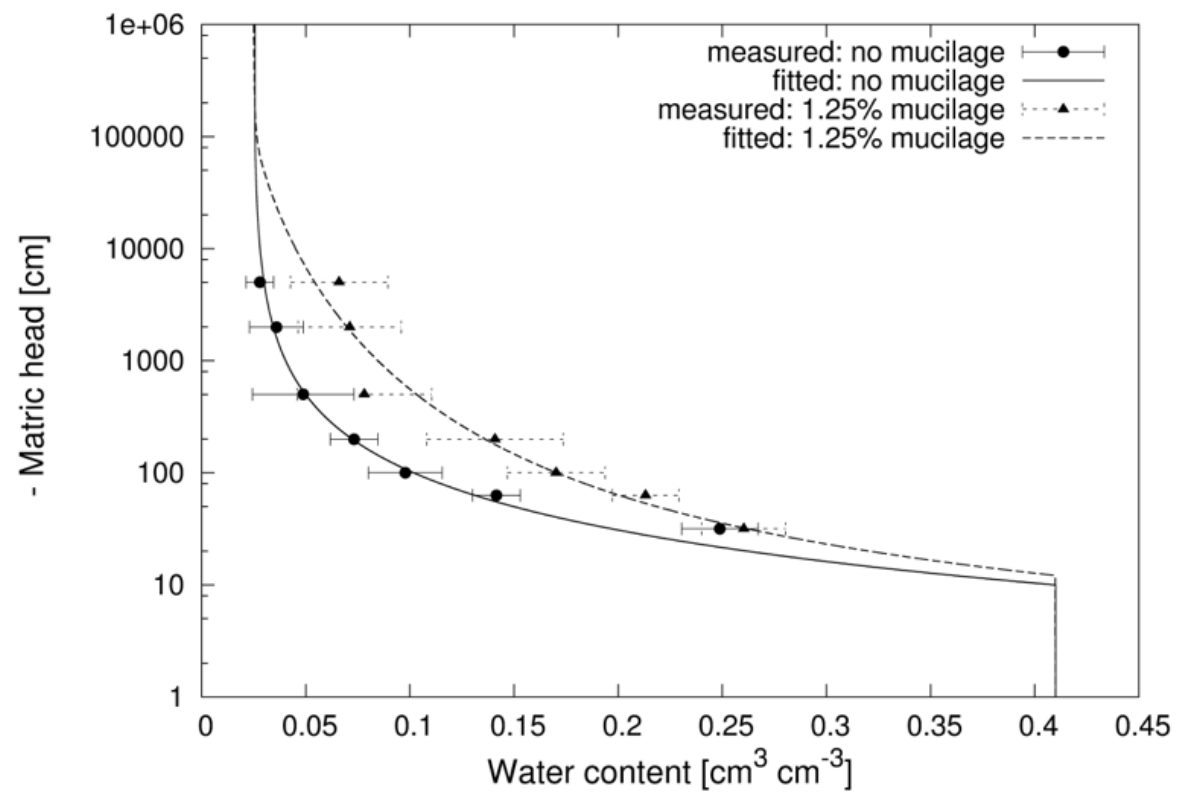

Figure 6.2: Effect of mucilage on the soil water retention curve. The figure shows that the soil mixed with mucilage retained more water than the control soil at any given water potential. The data points are averages of three replications. The error bars indicate the standard deviation of the measurements. The water retention curve of the soil without mucilage is fitted with the Brooks-Corey model (solid line). The data of soil with mucilage are fitted with Eqn.(6.5). The fitted parameters are presented in Table (6.1).

content were two independent variables and the water content had to be updated after each time step according to Eqn. 6.1.

Initial and boundary conditions correspond to the values of the experiments. The parameters of the model have been hand-fitted in order to obtain a good correlation to both experiments - i.e. the root pressure probe experiments and the mucilage drying experiment.

\subsection{Results}

Soil hydraulic properties $\quad$ Fig. 6.2 shows the effect of mucilage on the soil water retention curve. The results show that the soil mixed with mucilage retained more water than the control soil at any given water potential. The increased water content of the soil-mucilage mixture is the result of the water adsorbing capacity of mucilage. In fact, Lin et al. (1994) and Muñoz et al. (2012) showed that mucilage of chia seeds, upon wetting, formed a gel-like network with a remarkable capacity to adsorb water. 
Table 6.1: Brooks-Corey parameters of the soil water retention curve. The parameters were obtained by fitting the pressure plate apparatus measurements. The retention curve of the soil-mucilage mixture is parameterized according to Eqn. (6.4)).

\begin{tabular}{ccccrc}
$\theta_{s}$ & $\theta_{r}$ & $\lambda$ & $h_{0}[\mathrm{~cm}]$ & $\omega_{0}[\mathrm{~cm}]$ & $\beta$ \\
\hline 0.41 & 0.025 & 0.7 & -10 & $1.22 \times 10^{6}$ & 3.80 \\
\hline
\end{tabular}

Table 6.2: Parameters of the non-equilibrium model (Eqn.6.1)). The data are obtained from fitting the root pressure probe measurements and the drying of mucilage with the nonequilibrium model (Eqn.(6.1)).

\begin{tabular}{cccccc}
\hline Experiment & $a$ & $\gamma$ & $\tau_{0}[\mathrm{~s} \mathrm{~cm}]$ & $v$ & $d$ \\
\hline Root pressure probe & 100 & 4 & $1.43 \times 10^{4}$ & 566 & 1.4 \\
Injection of mucilage & 333 & 4 & $1.67 \times 10^{5}$ & 566 & 1.4 \\
\hline
\end{tabular}

The water retention curve for the soil was fitted using the Brooks-Corey parameterizations (Eqn. 6.4). The effect of mucilage is fitted using Eqn. (6.5). The fitted parameters are shown in Table 6.1

Drying rate of mucilage in soil To determine how long mucilage maintains the soil near the root wet, we injected $0.2 \mathrm{~g}$ mucilage in a soil with a water content of $0.04 \mathrm{~cm}^{3} \mathrm{~cm}^{-3}$ and we measured the local decrease in water content in the injection places. For comparison, we repeated the same experiment by injecting $0.2 \mathrm{~g}$ water in the soil. Fig. 6.3 shows the change in the volumetric water content after injection of mucilage and water. The results showed that the soil is drained much more slowly after mucilage injection than after water injection. One minute after injection, the soil water content was 2-3 times bigger than after water injection. The samples injected with mucilage approached equilibrium at a water content of $0.11 \mathrm{~cm}^{3} \mathrm{~cm}^{-3}$ in a period of approximately 24-48 hours. The samples injected with water reached a local water content of $0.05 \mathrm{~cm}^{3} \mathrm{~cm}^{-3}$ within 1-2 hours after injection. The higher water content at equilibrium for the mucilage injection is in line with the results of Fig. 6.2.

The experiment with water injection was fitted solving the Richards' equation in radial coordinates. The experiment with mucilage exudation was fitted solving the Richards' equation modified according to Eqn. (6.1)-(6.8)). The slow drainage of the mucilage is explained by two factors: the increased viscosity of mucilage (and the consequent decrease in soil hydraulic conductivity) and the low relaxation - left term of Eqn. (6.1). 


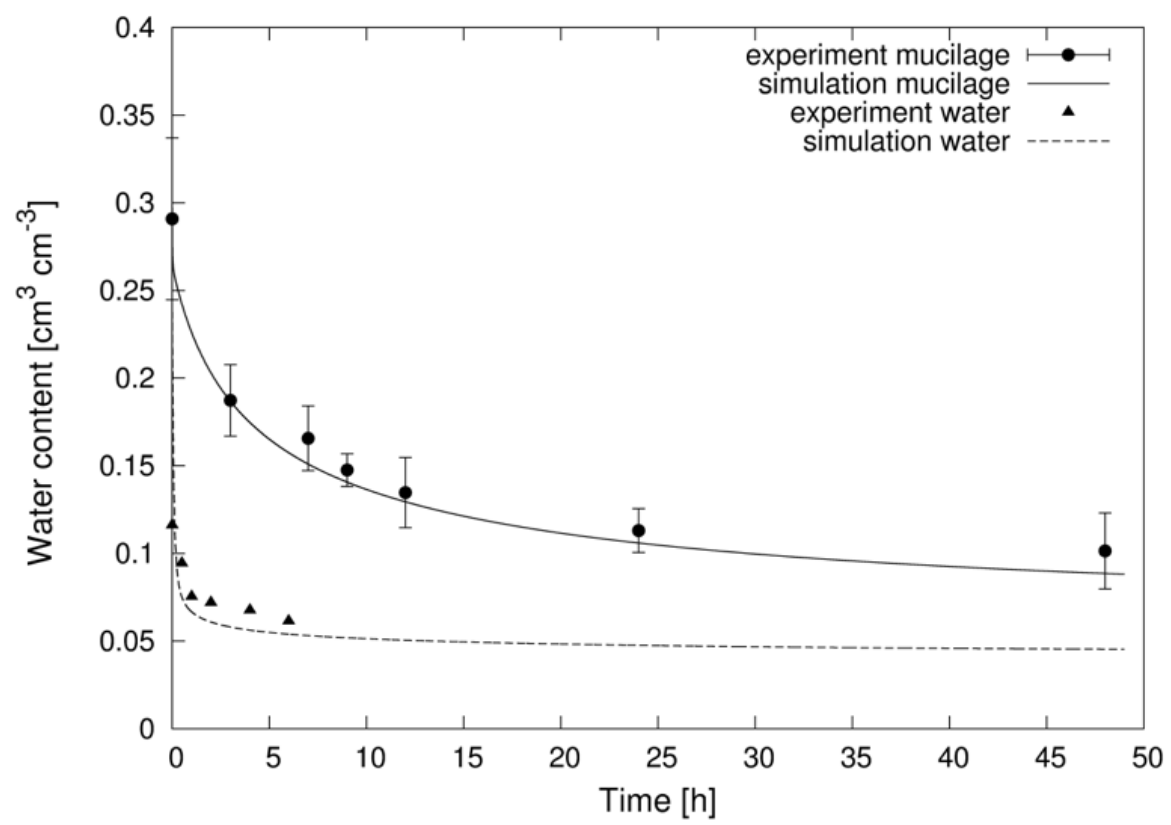

Figure 6.3: Change in the volumetric water content after injection of mucilage and water. The soil had an initial water content of $0.04 \mathrm{~cm}^{3} \mathrm{~cm}^{-3}$. The figure shows that the soil is drained much more slowly after mucilage injection than after water injection. The experiments were fitted solving the modified Richards' equation according to Eqn. (66.1)-(6.8)). The parameters are presented in Table 6.2 . 


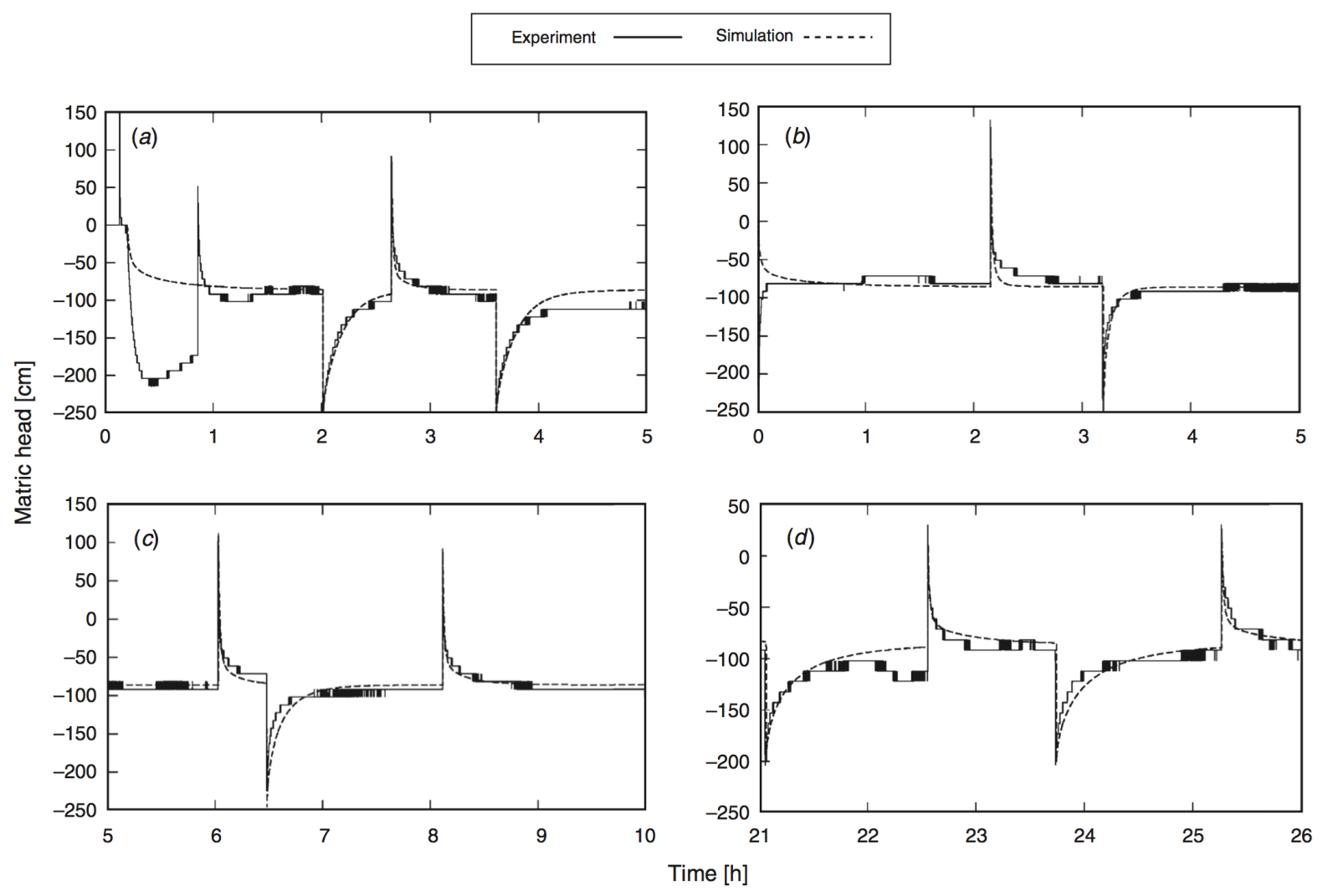

Figure 6.4: Pressure relaxation curves during several injection and suction pulses with artificial roots covered and not covered with mucilage. (a) Pressure curve for a root not covered with mucilage. After we placed the artificial roots in soil, we covered them with additional dry soil to achieve a good contact between root and soil. Due to the high water tension in the newly added soil, the root pressure dropped. When the pressure reached a constant value, we applied a series of injection and suction pulses over a period of $5 \mathrm{~h}$. The curves were fitted by solving the Richards' equation in radial coordinates. Pressure curves for an artificial root covered with mucilage over (b) 0-5 h, (c) 5-10 h and (d) 21-26 h (note the different scale in the $y$ axis in $(d)$ ). The drop in root pressure after placing the root in the soil was smaller and the pressure reached equilibrium faster. The pressure relaxations in roots covered with mucilage were initially much faster and then they became slower over time. The pressure relaxation curves were fitted solving Richards' equation modified according to Eqns (6.1)-(6.8)). The fitting parameters are shown in Table 6.2 . 
Pressure pulse experiment Fig. 6.4 shows the pressure relaxation curves during several injection and suction pulses into an artificial root covered with mucilage and into one placed in the control soil (no mucilage). During injection (positive pulses), the metal rode was quickly moved into the artificial root. The water pressure inside the root suddenly increased and induced a flow of water out of the root into the soil. As the water exited the roots, the root pressure decreased until it reached the water pressure in the soil near the root. During suction (negative pulses), the metal rode was quickly withdrawn, reducing the water pressure inside the root and inducing a water flow from the soil into the root.

Fig. 6.4 a shows the pressure curve during the experiments with the control soil (no mucilage around the artificial root). Before placing the artificial root into the soil, we measured the pressure relaxation in water. This gave the elasticity and the hydraulic conductivity of the artificial root. When we placed the artificial roots in soil, the pressure dropped sharply due to the high water tension in the soil. This caused an outflow of water from the artificial root to the soil. In addition to the low initial water potential in the soil, we also added 0.4 cm of dry soil on the top of the artificial root to achieve a good contact between the soil and the artificial root. As the newly added soil wetted, the root pressure partly recovered (around 30 minutes after placing the root in the soil). We waited around one hour until the pressure reached a constant value. Then we applied a series of injection and suction pulses over a period of 6 hours. The pressure relaxation in the suction experiment was slower than in the injection ones. This was caused by the decrease in water content near the root during the suction experiment and by the consequent reduction in hydraulic conductivity. We did not observe any significant change in pressure relaxation over time, indicating that the soil water content was close to equilibrium.

The curves were fitted by solving the Richards' equation in radial coordinates. To run the model the properties of root and soil are needed. The hydraulic conductance $K_{\text {root }}$ and the elasticity $\epsilon$ of the root pressure probe system were obtained by fitting a suction experiment when the root was placed in free water: The height of the peak leads to an elasticity of $\epsilon=$ $1.7510^{5} \mathrm{~cm} \mathrm{~cm}^{-3}$ and the relaxation time is related to the root conductance $K_{\text {root }}=5.7410^{-7}$ $\mathrm{cm}^{2} \mathrm{~s}^{-1}$ (value for the entire root surface).

Concerning the soil properties, the parameters of the water retention were determined by fitting the measured water retention curve (Table 6.1. Fig. 6.2). Additionally, we measured the saturated hydraulic conductivity of the soil $K_{\text {sat }}=8.4810^{-3} \pm 0.1310^{-3} \mathrm{~cm} \mathrm{~s}^{-1}$. The only parameter that was fitted was the tortuosity $L$. In the literature, an upper value of 2 
is recommended for the tortuosity of sandy soil. However, the simulation with $L=2$ did not match the observed curves. The bad fit was probably caused by a partial contact between the artificial root and sand. Indeed, during the measurements we realized how difficult was to place the root in uniform contact with the soil without leaving any gap. We included such complexity by introducing a thin layer of $1 \mathrm{~mm}$ thickness at the root surface where we set a different tortuosity. We obtained the best fit using $L_{b u l k}=2$ for the bulk soil and $L_{\text {int }}=4.8$ for the interface layer. This value is similar to the tortuosity of 5 obtained by Carminati et al. (2008) to describe the similar problem of partial contacts between soil aggregates. The fitting was very sensitive to the properties of the soil near the roots.

Fig. 6.4 b,c shows the pressure curves during one experiment with an artificial root covered with mucilage and placed into the soil as described above. Compared to the experiments without mucilage, the drop in root pressure after placing the root in the soil was much smaller and the pressure reached equilibrium faster. This was because the whole surface of the artificial root was covered with mucilage, that had a higher water potential than the soil. However, the root pressure at equilibrium was similar in the two experiments, suggesting that even when covered with mucilage, the root equilibrates with the water potential of the bulk soil. When the root pressure became constant, we applied a series of injection and suction pulses over a period of 24 hours. The pressure relaxation in roots covered with mucilage was much faster than in roots not covered with mucilage, in particular in the suction experiment. A quicker pressure relaxation indicates an easier flow of water across the soil-root interface. This proves that the water flow into the root was facilitated by mucilage.

Over time, the pressure relaxation became slower (Fig. 6.5). Fig. 6.5 shows the suction pulses from different samples at increasing time. After mucilage application, the pressure relaxation was quicker than in the soil without mucilage. After 24 hours, the relaxation times became similar. This was caused by the drainage of mucilage over time, as shown in Fig. 6.3 .

The pressure relaxation curves were fitted solving the Richards' equation modified according to Eqn. (6.1)-6.8)). The fitting parameters are shown in Table 6.2. The model was capable of reproducing well the evolution over time of the pressure relaxations. The fast pressure relaxation in the root covered with mucilage was caused by the high hydraulic conductivity of the soil-mucilage mixture next to the root. The evolution of the hydraulic conductivity in the soil next to the root for both cases with and without mucilage is plotted in Fig. 6.6. Fig. 6.6 shows that in both cases the hydraulic conductivity decreased after 


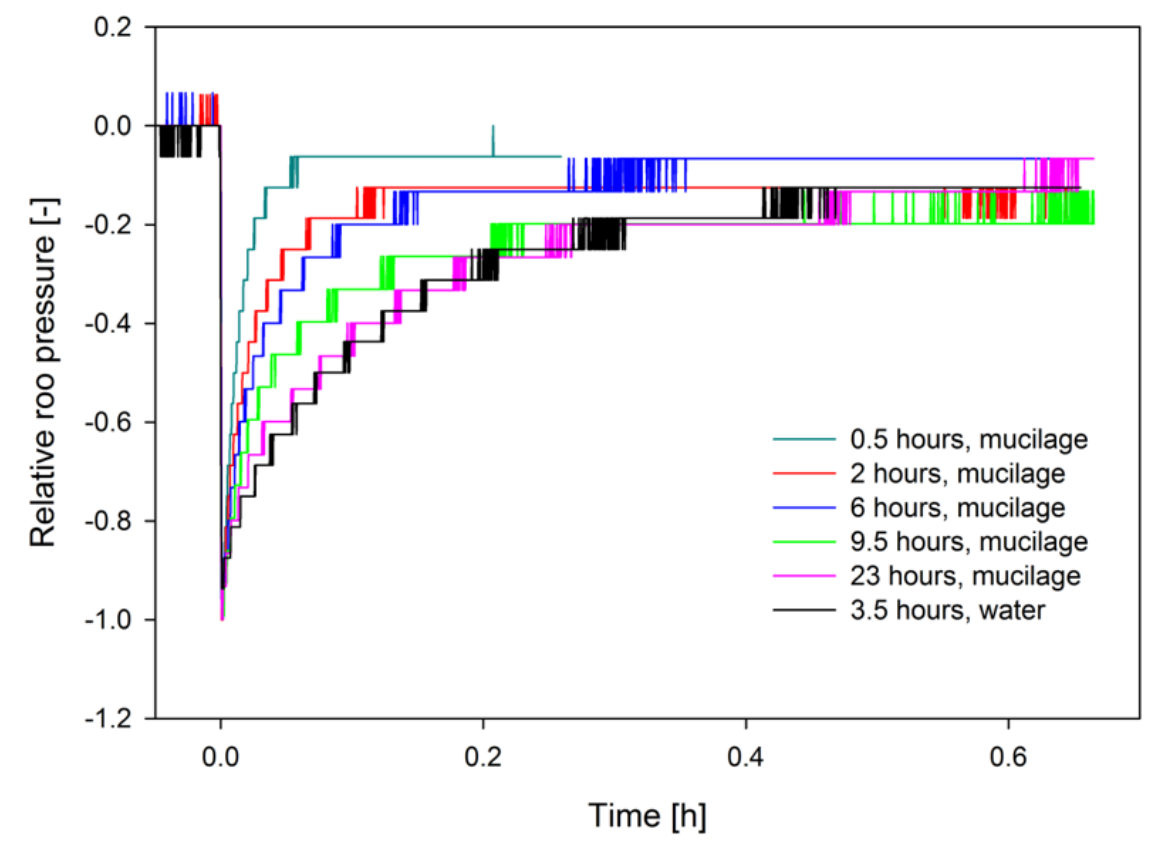

Figure 6.5: Pressure relaxation curves with artificial roots covered and not with mucilage at different times. After mucilage application, the pressure relaxation was much quicker than in the soil without mucilage. After 24 hours, the relaxation times became similar. This was caused by the drainage of mucilage over time. The data are normalized between zero and one for comparison. 


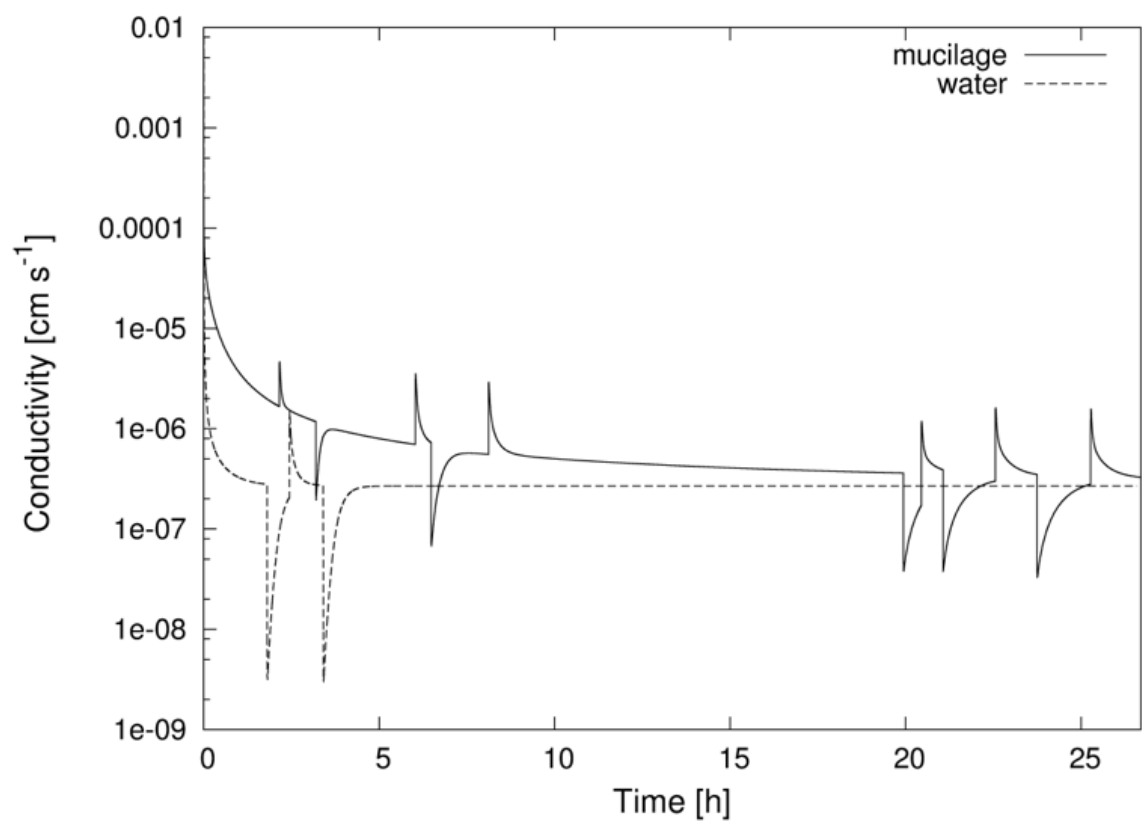

Figure 6.6: Evolution of the hydraulic conductivity in the soil next to the root for both cases, with and without mucilage. The figure shows that in both cases the hydraulic conductivity decreased after placing the root in soil. This was caused by the outflow of water from the root until the pressure reached equilibrium with the soil. Mucilage increased the hydraulic conductivity of the soil next to the root for the measured 24 hours. During the first 5 hours, the conductivity was increased of a factor of 10 times, which decreased to a factor of 2 after 24 hours. The decreasing differences over time were caused by mucilage drying.

placing the root in soil. This was caused by the outflow of water from the root until the pressure reached equilibrium with the soil. Mucilage increased the hydraulic conductivity of the soil next to the root for the measured 24 hours. During the first 5 hours, the conductivity was increased of a factor of 10 times, which decreased to a factor of 2 after 24 hours. The decreasing differences over time were caused by mucilage drying.

Interestingly, the model was able to explain the apparently contradicting results: the soil mixed with mucilage was drained much more slowly than the pure soil (Fig. 6.3), which seems to suggest a lower hydraulic conductivity of the soil mixed with mucilage. But on the other hand, the pressure pulse experiments show that mucilage allows water to flow more easily through a dry soil. These results would be difficultly understood and modeled when using the classical Richards equation. Our non-equilibrium model (Eqn. (6.1)) is able to reproduce both observations. Indeed, the two observations are not in contradiction. The slow drainage of the gel is caused by the slow diffusion of mucilage (and its solid network) 
through the pore space as well by the slow drying of mucilage and the consequent shrinking of its solid network. This was modeled introducing the non-equilibrium term on the left side of Eqn. 6.1). The consequent high water content in the soil-mucilage mixture results in an increase in the hydraulic conductivity of the soil near the root. In summary, the facilitated water flow to the root thanks to mucilage is the result of the slow spreading and drying of mucilage and the consequent increase in hydraulic conductivity.

Only two of the parameters used for the simulation of the pulse experiments (Fig. 6.4 b) were different from those used for simulating the experiment in Fig. 6.3. This was probably caused by the different way we injected mucilage in the two experiments. In the pulse experiments, we placed mucilage around the root and then we added dry soil on the top. This may have resulted in a preferential distribution of mucilage on the lateral sides of the root, which cannot be captured in our radial model. On the other hand, in the experiment of Fig. 6.4, we just injected mucilage in the soil. The two procedures may have resulted in different local soil density as well as a different spatial distribution of the gel.

\subsection{Discussion}

Our simple experiment showed that mucilage increased the unsaturated hydraulic conductivity of dry soils. Mucilage is therefore expected to favor root water uptake in relatively dry soils, when the hydraulic conductivity of the soil near the roots is expected to drop by several orders of magnitude and become the major resistance to the water flow into roots. By increasing the hydraulic conductance of the rhizosphere, mucilage helps plants to better capture water in dry conditions. The increase in hydraulic conductivity resulted from the capacity of mucilage to stay well hydrated. Specifically, we observed that: 1) mucilage increases the equilibrium water content of soil at any water potentials (Fig. 6.2); and 2) mucilage does not quickly spread into the soil and it is slowly drained (Fig. 6.3). Both results confirm the hypothesis of Young (1995), that mucilage increases the water content of the rhizosphere compared to that of the bulk soil. The results do not contradict the measurements of McCully and Boyer (1997), who showed that mucilage loses most of its water at high water potentials. The remaining volume of water retained by mucilage at more negative water potentials accounts for the increase in water content of about 0.05 (Fig. 6.2).

Our experiments were carried out using artificial roots and mucilage collected from chia seeds. This choice was functional to perform well controlled experiments, avoiding the complexity of variable root conductivity (Steudle, 2000; Maurel et al., 2008). Additionally, it 
can be argued that mucilage from chia seeds has different properties than mucilage exuded by plant roots in the field. However, following a similar reasoning, we argue that it is unlikely that mucilage properties are equal in all plant species, soil types and environmental conditions. We observed that mucilage from chia seeds has a similar behavior as that from maize and lupine: it forms a gel when immersed in water and it turns hydrophobic after drying. A similar chemical composition was also reported (Lin et al., 1994). Our conclusions on the effect of mucilage on root water uptake can therefore be generalized to more realistic cases, although the extent of these effects will vary among plants, soils and environmental conditions. For instance, we carried out our experiments in sandy soils. We expect that in more loamy and clayey soils the effects of mucilage on the soil hydraulic conductivity would be smaller.

We showed that at a water content of 0.03-0.04 mucilage maintains the soil wet and well conductive for a period of 24 hours. Afterwards, mucilage is drained and its hydraulic conductivity approaches that of the original soil. The effects of mucilage are therefore highly dynamic and are expected to be more significant in the young root segments, while old root segments are likely to be covered with old and drained mucilage. Besides being drained, mucilage can be degraded by microorganisms, which themselves are covered by extracellular polymeric substances (EPS) (Chenu and Roberson, 1996; Or et al., 2007). Drying, degradation and alteration of mucilage will therefore result in varying hydraulic properties of the rhizosphere along the root system. This conclusion follows the point of view of Watt et al. (2006), who reported that rhizosphere properties change as a function of distance from root tips and growth rate. Similarly, our findings support the hypothesis of Carminati and Vetterlein (2013), who suggested that rhizosphere plasticity helps plants to better adapt to the heterogeneous distribution of soil resources, like water and nutrients. Our results are also in line with former observations of wet rhizosphere around young root segments (Watt et al., 1994).

Drying and potential degradation of mucilage and the consequent heterogeneity of rhizosphere properties along roots complicate the up-scaling of our concept to the plant scale. According to our results, freshly exuded mucilage facilitates root water uptake for a period of at least 1 day. Assuming that roots grow at a velocity of $2 \mathrm{~cm} \mathrm{day}^{-1}$, we expect that mucilage facilitates the water flow into the first $2 \mathrm{~cm}$ near the root tip. The effect of mucilage at the plant scale depends on the capacity of the root system to efficiently transport water from the most distal root segments to the shoots. To upscale the effects of mucilage is therefore 
needed to know the properties of the root architecture (Draye et al., 2010). Interestingly, our results suggest that the positive effects of mucilage on root water uptake increase when roots grow faster.

In conclusion, we conducted a simplified experiment to test the effects of mucilage on water flow in dry soils. The results demonstrated that mucilage facilitated the water flow in dry soils and support our hypothesis that mucilage exudation helps plant roots to take up water from dry soils. Other studies are necessary to estimate the effects of mucilage exudation in more realistic scenarios and how these effects vary among plant species, soil types and growing conditions. Possibly, these studies should also consider the effects on nutrient uptake and on microorganisms living in the rhizosphere. At the moment, what we can conclude is that mucilage has the potential to increase the capability of young root segments to capture water from soils, in particular when the soil is drying. Such characteristic potentially helps plants to use soil resources and survive drought spells. A better understanding of mucilage and rhizosphere interactions may therefore bring to new strategies to decrease drought stress in plants. 


\title{
$7 \quad$ Simulation of root water uptake under consideration of non- equilibrium dynamics in the rhizosphere
}

written by Eva Kröner, Mohsen Zarebanadkouki, Marco Bittelli, Andrea Carminati, submitted to Water Resources Research (Kroener et al., submitted 2016)

\begin{abstract}
The narrow region of soil around roots, the so-called rhizosphere, defers in its hydraulic properties from the bulk soil. The rhizosphere hydraulic properties primarily depend on the drying and wetting rate of mucilage, a polymeric gel exuded by plant roots. Under equilibrium conditions mucilage increases the water holding capacity. Upon drying mucilage turns hydrophobic and makes the rhizosphere temporarily water repellent.

There are several models of root water uptake, from analytical models of water flow to a single root to complex numerical models that consider the root architecture. Most of these models, however, do not account for the specific hydraulic properties of the rhizosphere.

Here we describe a single-root model that includes the altered hydraulic properties of the rhizosphere due to mucilage exudation. We use the model to reproduce existing experiments reporting unexpected and puzzling hysteresis in the rhizosphere, which could not be explained under the assumption of homogeneous hydraulic properties. In our model the hydraulic properties depend on the concentration of mucilage. This enables a continuous transition from the bulk soil to the root surface. We assumed that: (a) mucilage increases the water holding capacity in equilibrium conditions, (b) hydrophobicity, swelling and shrinking of mucilage cause a non-equilibrium relation between water content and water potential and (c) mucilage reduces the mobility of water molecules in the liquid face resulting in a lower hydraulic conductivity at a given water content.

Our model reproduces well the experiments and suggests that mucilage softens drought stress in plants during severe drying events.
\end{abstract}

\subsection{Introduction}

The ability of plants to extract water from the soil, depends on the hydraulic properties of the roots, the soil and in particular the soil in the immediate vicinity of the roots. Under dry conditions root water uptake becomes limited by the hydraulic resistance of a narrow region of soil around roots, the rhizosphere. The hydraulic properties of the rhizosphere are 
affected by mucilage, a polymeric gel exuded by the root tips of most of the plants. Including mucilage in models of root water uptake is challenging but it is needed for properly describing the water content dynamics in the rhizosphere during drying and wetting cycles.

Advanced models of root water uptake have emerged over the last decades. These models range from macroscopic models where root water uptake is proportional to an empirical sink term (Feddes et al., 1976; Jarvis, 1989; Clausnitzer and Hopmans, 1994) to microscopic analytical models (Gardner, 1960) and finally to numerical models simulating the threedimensional water flow in soil and roots (Roose and Fowler, 2004, Doussan et al., 2006. Javaux et al. 2008). With a few exception, most of these models do not account for altered hydraulic dynamics of the rhizosphere.

Experiments with soil samples mixed with mucilage (Kroener et al., 2014b) showed that: (1) mucilage increases the water content in the rhizosphere when in equilibrium at a given soil matric potential. (2) It contains lipids - long-chained molecules (Read et al., 2003), that result in an exposed hydrophobic surface when dry and that explain water repellency found in rhizosphere at low water contents (Moradi et al., 2012, Ahmed et al., 2015c). (3) A heterogeneous distribution of root exudates in the pores and swelling and shrinking dynamics of mucilage result in a non-equilibrium relation between water content and water potential (Carminati, 2012). (4) The higher is the concentration of mucilage the more viscous is the liquid phase and the lower is the mobility of water molecules. As a result the presence of mucilage in the rhizosphere reduces the hydraulic conductivity at a given water content.

The interplay between these effects results in a dynamic hydraulic behaviour of the rhizosphere: while under equilibrium conditions the water content in rhizosphere is higher than in bulk soil (Carminati et al., 2010; Moradi et al. 2011), mucilage hydrophobity together with a slow rewetting rate of mucilage can lead to a reduced rhizosphere water content for some days after irrigation (Carminati et al. 2010).

The heterogeneous distribution of mucilage together with a percolation model Kroener et al., 2015, Benard et al., 2015) can provide an explanation at the pore scale for these nonequilibrium dynamics: when the mucilage-covered pores become dry they turn hydrophobic. Now the rewetting of these pores depends on the swelling rate of mucilage. Pores that are not filled with mucilage can rewet almost immediately when in contact with water. Near the percolation threshold, when the wettable pores form a connected network that spans throughout the rhizosphere, water can flow across the rhizosphere while the water content within the rhizosphere remains low for a longer period of time. 
In our previous study (Kroener et al., 2014b) we proposed a model that describes how a certain concentration of mucilage alters the bulk soil hydraulic functions. Now we apply and test the model to simulate existing observations of water flow across the rhizosphere. In the model the hydraulic properties are functions of mucilage concentration. In our implementation the concentration of mucilage gradually increases towards the root surface. As a result also the hydraulic properties change gradually across the rhizsophere.

In the initial part of the paper we provide a short introduction of the conceptual model (Kroener et al. 2014b) and the equations employed. Then, we use the model to simulate experiments from the literature that indicate altered hydraulic dynamics of the rhizosphere around roots of various ages (Fig. 7.1). Indeed, it is expected that the rhizosphere properties change over time. Such changes are possibly caused by daily drying/wetting of mucilage, its spreading through the rhizosphere and mucilage biodegradation.

We fitted experiments with plants and roots of varying age and varying soil conditions. The first experiment shows that shortly after exudation young mucilage can increase the hydraulic conductivity of the root-soil interface (Ahmed et al. 2014). Carminati et al. (2010) measured the slow rewetting dynamics of mucilage after irrigation and found that mucilage from older roots has slower rewetting dynamics than young mucilage. Zarebanadkouki et al. (2013) monitored rewetting dynamics in the rhizosphere as function of distance from the roots. And finally Passioura (1980) observed a puzzling hysteresis during cycles of increasing/decreasing transpiration with wheat plants.

\subsection{Material and Methods}

\subsubsection{Hydraulic properties of the rhizosphere}

In our simulations we use the model presented by Kroener et al. (2014b) to simulate observations of water flow across the rhizosphere. Objective of the model is to determine the rhizosphere hydraulic properties from bulk soil hydraulic properties and mucilage concentra-

tion $c_{t o t}\left[\mathrm{~g} \mathrm{~cm}^{-3}\right]$ (dry weight of mucilage per unit volume of soil). As it is expected that $c_{t o t}$ decreases continuously from the root surface to the bulk soil, similarly it is expectable that the soil hydraulic properties change continuously as well. The model has been applied to wetting and drying experiments of mucilage-soil mixtures (Kroener et al., 2014b) and to a root water uptake experiment with a suction cup as artificial root (Ahmed et al., 2014). The model considers that mucilage leads to

(a) a non-equilibrium relation between water potential and water content 


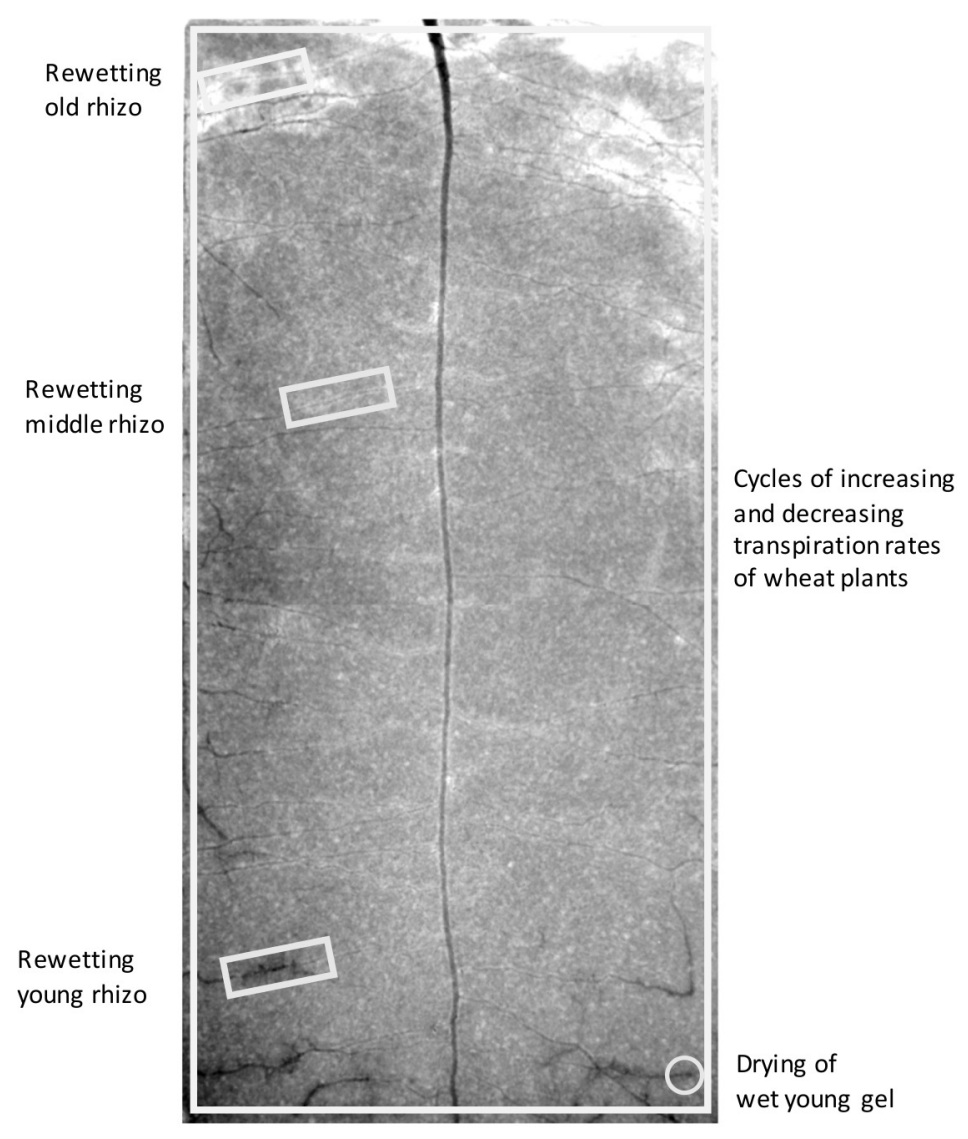

Figure 7.1: Water content distribution shortly after irrigation. The light grey boxes indicate the roots that correspond to the stage of the roots in the simulated experiments. As an illustration we used a lupine for this figure. Note that not all the simulated experiments are based on lupines. 
(b) an increase in soil water holding capacity when the system is in hydraulic equilibrium

(c) a decrease in hydraulic conductivity when kept at same water content as bulk soil.

It is assumed that in each elementary volume of the rhizosphere a certain fraction of pores is filled with mucilage and that the other pores do not contain mucilage. We defined the non-mucilage-filled pore ratio as $R=1-\frac{V_{G P}}{\Phi}$, where $\Phi\left[\mathrm{cm}^{3} \mathrm{~cm}^{-3}\right]$ is the soil porosity and $V_{G P}\left[\mathrm{~cm}^{3} \mathrm{~cm}^{-3}\right]$ is the gel-filled pore volume per unit volume of soil. It is imposed that $V_{G P}$ decreases from a value close to $\Phi$ near the root surface to zero in the bulk soil. That means that $R$ increases from a small value at the root surface to $R=1$ in the bulk soil.

The volumetric water content $\theta\left[\mathrm{cm}^{3} \mathrm{~cm}^{-3}\right]$ is the sum of the water content in the nongel-filled space $\theta_{b u}$ and the water content in the mucilage-filled space $\theta_{M}$ weighted by $R$ :

$$
\theta=R \theta_{b u}+(1-R) \theta_{M}
$$

The swelling and shrinking dynamics inside the mucilage-filled pores lead to a decoupling of water content $\theta$ and water potential $\psi[\mathrm{cm}]$. The following equation describes the change in total water content as a function of the dynamics in the two compartments:

$$
\frac{\partial \theta}{\partial t}=R C_{b u}(\psi) \frac{\mathrm{d} \psi}{\partial t}+(1-R) \frac{1}{\tau}\left[\psi-\psi^{e q}\left(\theta_{M}\right)\right]
$$

where $t[\mathrm{~s}]$ is time, $C_{b u}\left[\mathrm{~cm}^{-1}\right]$ is capacity of the bulk soil, $\psi^{e q}\left(\theta_{M}\right)$ is the water potential that would be reached under equilibrium conditions when the mucilage-filled space was kept at a water content of $\theta_{M}$. The parameter $\tau$ can be seen as the relaxation time of the process: the larger it is, the longer it takes for the water content to reach the equilibrium.

$R$ depends on total mucilage concentration $c_{t o t}\left[\mathrm{~g} \mathrm{~cm}^{-3}\right]$ (dry weight of mucilage per dry weight of soil): the more gel there is the smaller is the ratio of non-mucilage-filled pores $R$. Requirements to the function $R\left(c_{t o t}\right)$ are:

1. $R\left(c_{t o t}\right)$ is monotonically decreasing with increasing concentration of mucilage

2. $R\left(c_{t o t}\right)=1$ if $c_{t o t}=0$

3. $0 \leq R\left(c_{t o t}\right)<1$ if $c_{t o t}>0$

A simple function that fullfills these conditions is a decreasing exponential function:

$$
R\left(c_{t o t}\right)=e^{-a c_{t o t}}
$$

where $a\left[\mathrm{~cm}^{3} \mathrm{~g}^{-1}\right]$ is a fitting parameter. 
We expect that the relaxation time $\tau$ depends on $\theta_{M}$ : the drier the mucilage-filled pores, the higher the concentration of mucilage in the liquid phase, the stronger the bonds between mucilage polymers and the longer it will take for the system to go back to equilibrium. This is described by a simple polynomial:

$$
\tau\left(\theta_{M}\right)=\theta_{M}^{-\gamma} \tau_{0}
$$

where $\gamma$ and $\tau_{0}$ are fitting parameters.

The hygroscopic nature of mucilage decreases the water potential of a given volume of water. In other words: under equilibrium conditions mucilage increases the water holding capacity of bulk soil at any negative water potential. Similar to an osmotic potential that is a function of concentration of solutes, we assume that the water potential is reduced by a term related to the average mucilage concentration in the liquid phase of the mucilage-filled pores $c_{M}=\frac{c_{t o t}}{\theta_{M}(1-R) \rho_{w}}$ with density of mucilage $\rho_{w} \approx 1 \mathrm{~g} \mathrm{~cm}^{-3}$ according to:

$$
\theta_{M}^{e q}(\psi)=\theta_{b u}\left(\psi+\omega_{0} c_{M}^{\beta}\right)
$$

where $\omega_{0}$ and $\beta$ are fitting parameters. For the osmotic potentials of diluted solutions we expect $\beta=1$ - note that for diluted solutions where solutes do not interact with each other. Polymers forming mucilage, however, bind to each other creating a network. So we allowed also values higher than 1 for $\beta$.

When mucilage becomes dry its viscosity increases and the mobility of water molecules within the gel decreases. In our model the rhizosphere hydraulic conductivity is equal to the bulk soil hydraulic conductivity (at the same water content) scaled by a factor $\mu$ :

$$
K_{\mathrm{rh}}(\theta, \mu)=\frac{\mu_{w}}{\mu} K_{\mathrm{bu}}(\theta)
$$

where $\mu_{w}$ is the viscosity of water and $K_{\mathrm{rh}}$ and $K_{\mathrm{bu}}$ are the hydraulic conductivities in the rhizosphere and bulk soil, respectively. We assume that the scaling factor $\mu$ is function of mucilage concentration in the liquid phase $c=\frac{c_{t o t}}{\theta \rho_{w}}$ with density of water $\rho_{w} \approx 1 \mathrm{~g} \mathrm{~cm}^{-3}$. In our model $\mu$ follows a similar law as the viscosity of polymeric solutions (Morris et al., 1981):

$$
\mu(c)=\mu_{w} \max \left\{\begin{array}{l}
{\left[\frac{c}{c_{0}}\right]^{d_{0}}+1} \\
{\left[\frac{c}{c_{1}}\right]^{d_{1}}}
\end{array}\right.
$$

where $c_{0}, d_{0}, c_{1}$ and $d_{1}$ are fitting parameters. Here we do not distinguish between mucilagefilled pore space and non-mucilage-filled pore space, and we calculate an effective conductivity by fitting $c_{0}, d_{0}, c_{1}$ and $d_{1}$. 
Carminati et al. (2010) have found that the region within circa $r_{r h i z}=2 \mathrm{~mm}$ distance from the root differed from the bulk soil in its hydraulic behaviour. In our study we assume that the total concentration of mucilage decreases linearly with distance from the root to a value of 0 at $r_{r h i z}=2 \mathrm{~mm}$ :

$$
\begin{aligned}
& c_{\text {tot }}=\frac{r_{\text {rhiz }}-r}{r_{\text {rhiz }}-r_{\text {root }}} c_{0} \quad \text { if } r<r_{r h i z} \\
& c_{\text {tot }}=\quad 0 \quad \text { if } r \geq r_{r h i z}
\end{aligned}
$$

where the total concentration of dry gel at the root surface is assumed to be $c_{0}=1 \mathrm{mg} / \mathrm{cm}^{-3}$, based on the approximate estimation of $0.1 \%$ (Carminati and Vetterlein, 2013).

\subsubsection{Implementation of root water uptake model}

To simulate root water uptake we used a single-root approach: the complex 3-dimensional root architecture is simplified into a single root. Now it is sufficient to solve the Richard's equation in the radial coordinate $r$. Drawback of this approach is that variations within the root system, e.g. various root types and root ages, and the root architecture are not represented. The one-dimensional radial Richard's equation is:

$$
\frac{\partial \theta}{\partial t}=\frac{1}{r} \frac{\partial}{\partial r} r K \frac{\partial}{\partial r} \psi
$$

To numerically solve Eqn. (7.10) we started with the Python-program implemented by Bittelli et al. (2015) for water flow in soils: at first, the mass balance is formulated for each node, where the gradients are approximated using finite differences. Then the mass balance equation is solved with a Newton method using Thomas-Algorithm to solve the linear equations. Because of the high non-linearity of the problem, an implicit Euler scheme was chosen for the time discretization.

We modified the program to account for the rhizosphere hydraulic properties. Eqs. (7.2) and 7.10 give:

$$
\begin{aligned}
0 & =R \frac{\mathrm{d} \theta_{b u}(\psi)}{\mathrm{d} t}+(1-R) \frac{1}{\tau}\left[\psi-\psi^{e q}\left(\theta_{M}\right)\right] \\
& -\frac{1}{r} \frac{\partial}{\partial r} r K(\theta) \frac{\partial}{\partial r} \psi
\end{aligned}
$$

In each Newton iteration this equation is solved for $\psi$. After each Newton iteration $\theta_{M}$ and 
$\theta$ are updated using:

$$
\begin{aligned}
\frac{\partial \theta_{M}}{\partial t} & =(1-R) \frac{1}{\tau}\left[\psi-\psi^{e q}\left(\theta_{M}\right)\right] \\
\frac{\partial \theta}{\partial t} & =R \frac{\partial \theta_{b u}(\psi)}{\partial t}+\frac{\partial \theta_{M}}{\partial t}
\end{aligned}
$$

Adaptive time-stepping guaranteed that in each time step the maximum number of iterations, the maximum change in water content and the maximum change in matric potential were smaller than a certain tolerance. For the spatial discretization, we chose a grid of 100 cells with cell sizes increasing geometrically with distance to the root.

\subsubsection{Simulated experiments}

We simulated five experiments of root water uptake (Ahmed et al., 2014, Carminati et al., 2010; Carminati, 2013; Zarebanadkouki et al., 2015; Zarebanadkouki and Carminati, 2014; Passioura, 1980). The experiments varied regarding the age of the rhizosphere but also in their experimental set-up, the measured variables and plant and soil hydraulic conditions. All of these experiments indicate hydraulic dynamics in the rhizosphere that could not be explained by the assumption of homogeneous properties around the roots. In the following subsections we describe the idea and the concept of the experiments; a more detailed description is provided as supplementary material.

Drying of fresh mucilage Ahmed et al. (2014) found that in dry soil exudation of mucilage increases the hydraulic conductivity of the root-soil interface, and facilitates root water uptake for at least one day after exudation of fresh mucilage. They connected a suction cup, referred to as artificial root, to a root pressure probe (Liu et al., 2009) and applied several suction and injection pulses into the root. For each of the pulses a pressure transducer recorded the relaxation of the root pressure over time until it equilibrated with the soil.

The conductivity of the root-soil continuum is related to the relaxation of the root pressure: the higher the conductivity, the faster the relaxation of the pulse. They compared measurements with the artificial root covered with mucilage extracted from chia seeds to measurements with artificial root without mucilage and found that mucilage increases the conductivity by a factor of more than ten during the first hours after exudation (Fig. 6 in Ahmed et al. (2014)).

Rewetting of dry young/old rhizosphere Carminati et al. (2010) monitored the water content distribution around roots of lupine plants during a drying and wetting cycle. They 
found that during drying the rhizosphere had a higher water content than the bulk soil. The samples where irrigated by capillary rise after five days when water content in bulk soil and rhizosphere were around 0.01-0.02. In all the experiments bulk soil rehydrated relatively fast while the rhizosphere stayed temporarily dry.

The rhizosphere of roots younger than one week rewetted within one hour (Carminati, 2013). For older root segments the rewetting of the rhizosphere took much longer, around two days (Carminati et al., 2010).

Water content distribution during rewetting of dry rhizosphere Zarebanadkouki et al. (2015) used neutron radiography to quantify at higher spatial and temporal resolution the rewetting dynamics of the region around roots after irrigation. Plants and soil were the same as used in the experiment described above - i.e. lupines in a sandy soil. At all times water content distribution followed a gradual transition from the water content in the bulk soil to the water content at the root surface, consistent with the idea that mucilage concentration gradually increases from the bulk soil to the root surface. The authors have measured the profiles of water content towards the roots for 2-3 hours after irrigation. The samples were irrigated from the top.

Wheat xylem pressure during increasing and decreasing transpiration Passioura (1980) investigated the relation between transpiration rate and water potential in the leaf xylem of young wheat plants: in his experiment he controlled the transpiration rate by varying air humidity and illumination. Transpiration was increased in several steps to a maximum value and then transpiration was decreased following the same steps. During each of these steps, the pressure difference between leaf xylem and soil was measured as it follows: the soil and roots of the plant was inside a pressure chamber, while the above ground part of the plant was at atmospheric pressure. One leaf was cut and connected to a capillary. The pressure in the chamber was then adjusted in such a way that the meniscus of the capillary was in equilibrium with the atmosphere. The applied pressure was equal to the difference in pressure between the soil and leaf xylem and it contains the information on the pressure dissipation across the soil-plant continuum at varying transpiration rates and soil water contents.

The transpiration cycles were repeated during a drying period until the plant showed the first wilting symptoms. While the experiments in wet soil could be reproduced well with the classic Richards equation, Passioura (1980) observed a puzzling hysteresis in the relation between transpiration rate and leaf xylem water potential in dry soils. A similar hysteresis 
Table 7.1: Parameter set obtained by fitting the simulated results to the experimental data.

(Unit of $\mathrm{c}$ is $\left[\mathrm{g} \mathrm{cm}^{-3}\right]$ )

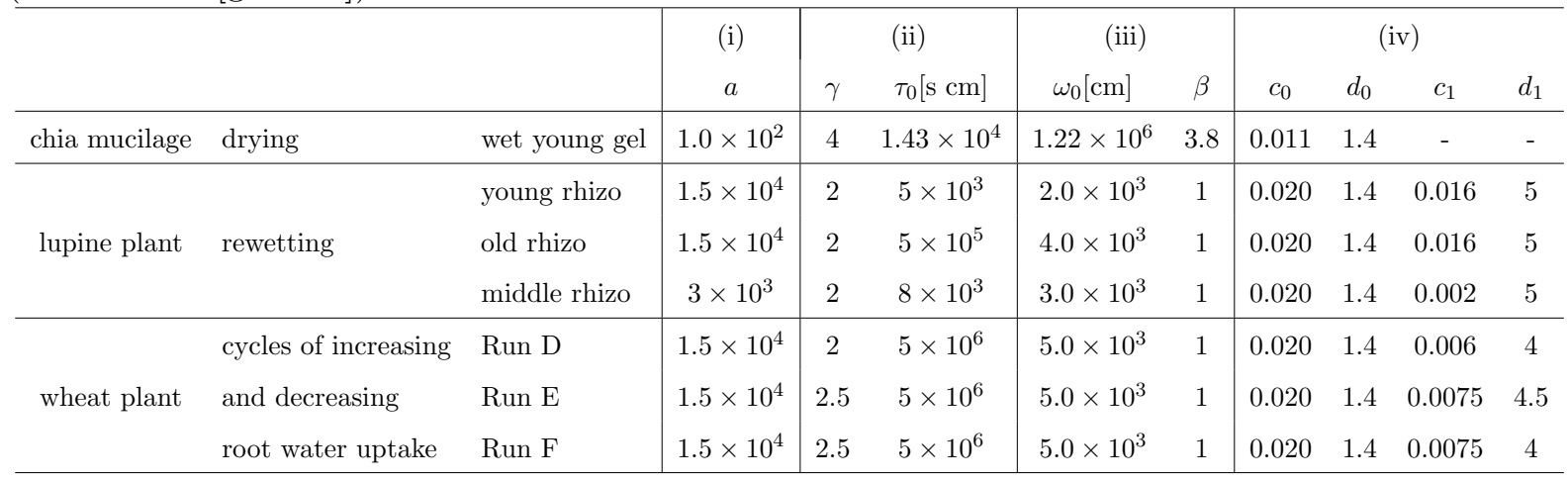

has been more recently observed by Deery et al. (2013) using a similar approach.

Our intention regarding the simulations of Passioura's experiment was to show that this unexpected hysteresis could be reproduced if time-dependent drying and wetting of mucilage in the rhizosphere are considered. However, also other factors, e.g. accumulation of salts or three-dimensional root-architecture considerations, might explain and contribute to this hysteretical behaviour.

\subsection{Results}

By fitting the simulations to the experimental data we obtained a set of parameters for each of the experiments (Tab. 7.1). In the following subsections we present the fitting of the individual experiments.

Drying of fresh mucilage Fitting the pressure relaxation measured with the root pressure probe after injection and suction pulses gives an estimation of the hydraulic conductivity of the rhizosphere at the root surface (Fig. 7.2 a). Shortly after exudation of mucilage the conductivity at the root surface is more than one order of magnitude larger than in the case without mucilage. In this experiment the hydraulic conductivities for the case with and without mucilage reach a similar value after around one day.

The fitted parameters suggest that for a range of matric potentials $-100 \mathrm{~cm}<\psi<$ $-10 \mathrm{~cm}$ under equilibrium conditions the rhizosphere hydraulic conductivity is lower than the bulk soil hydraulic conductivity (Fig. 7.2p). During drying the non-equilibrium dynamics result in the rhizosphere hydraulic conductivity being higher than the bulk soil hydraulic conductivity at the same water potentials. This increase is sensitive to parameter group (ii) (Tab. 7.1) describing the slow relaxation dynamics. 

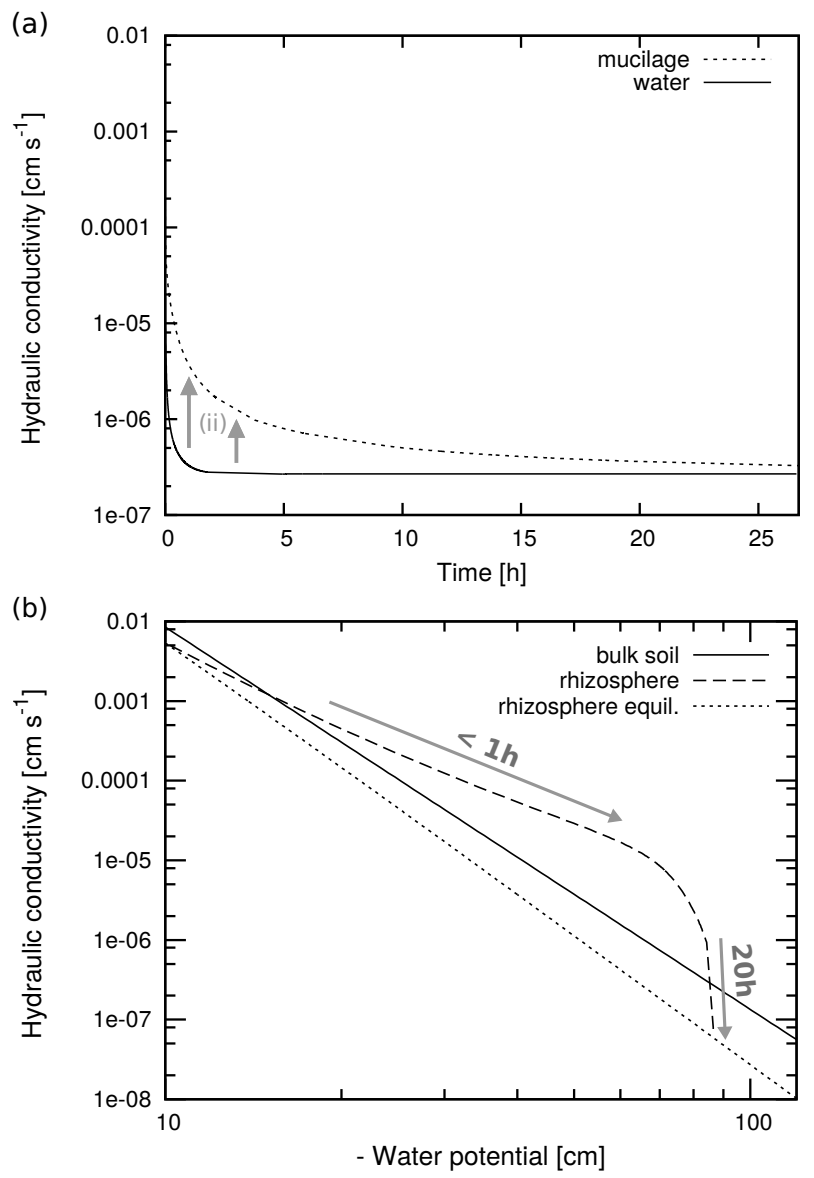

Figure 7.2: (a) Estimated hydraulic conductivity of the rhizosphere after placing mucilage respectively water in a small region around the root [adapted from Ahmed et al. (2014), Fig. 6]. The data have been obtained by fitting the measured root pressure of the artificial root. (b) Estimated hydraulic conductivity of bulk soil and in the rhizosphere at root surface both during the simulation and under equilibrium conditions. 
Rewetting of dry young/old rhizosphere Rewetting of dry rhizosphere of a young root takes around an hour while rhizosphere of old roots needs a few days to rewet (Fig. $7.3 \mathrm{a}, \mathrm{b}$ ). By fitting the data we found that the parameter group (iii) (Tab. 7.1) controls the increase of water content in rhizosphere compared to the bulk soil during the drying phase of the first four days. Water content changes after irrigation are sensitive to the parameter group (ii) (Tab. 7.1), which describes the relaxation dynamics. $a$ has a strong impact on the small increase of rhizosphere water content immediately after irrigation (Fig. $7.3 \mathrm{~b}$ at day 6 ), $\tau_{0}$ influences the relaxation rate of rhizosphere water content during the days after irrigation and $\gamma$ defines the shape of the increase of water content.

Water content dynamics in the rhizosphere under dry conditions (from day 4 to day 6) are sensitive to parameter group (iv) (Tab. 7.1) describing the reduced hydraulic conductivity. Additionally, these parameters influence the water potential at the root surface. The parameters can be conditioned considering that the plant already showed some wilting symptoms before irrigation, which suggests that the matric potential at the root surface could have been around -1.5 MPa.

At high water potentials $(\psi>-200 \mathrm{~cm})$ the equilibrium rhizosphere hydraulic conductivity (Fig. 7.3 c,d) is similar to the bulk soil hydraulic conductivity, and it is even slightly higher due to the higher water content (parameter group (iii), Tab. 7.1), until it drops at around $-150 \mathrm{~cm}$. During rewetting, the non-equilibrium dynamics result in the rhizosphere hydraulic conductivity being much lower than bulk soil hydraulic conductivity. In the simulation of rewetting of old rhizosphere (Fig. 7.3 d) there is a peak in water potential at a hydraulic conductivity of around $10^{-10} \mathrm{~cm} \mathrm{~s}^{-1}$. This peak is caused by the spatial non-equilibrium dynamics when at a point mucilage takes up water and applies suction to other parts of the rhizosphere.

\section{Water content distribution during rewetting of dry rhizosphere The simulation} could reproduce the experimental results where at all time steps a gradual transition of water content from bulk soil to root surface are visible (Fig. 7.4a). During rewetting the hydraulic conductivity of the rhizosphere at the root surface is always below the one of the bulk soil at the same water potential (Fig. $7.4 \mathrm{~b}$ ).

Wheat xylem pressure during increasing and decreasing transpiration The relation between transpiration rate and water potential in the leaf xylem (Fig. 7.5 ) shows a hysteresis which can be reproduced by our model (sensitive to parameter group (ii), Tab. 

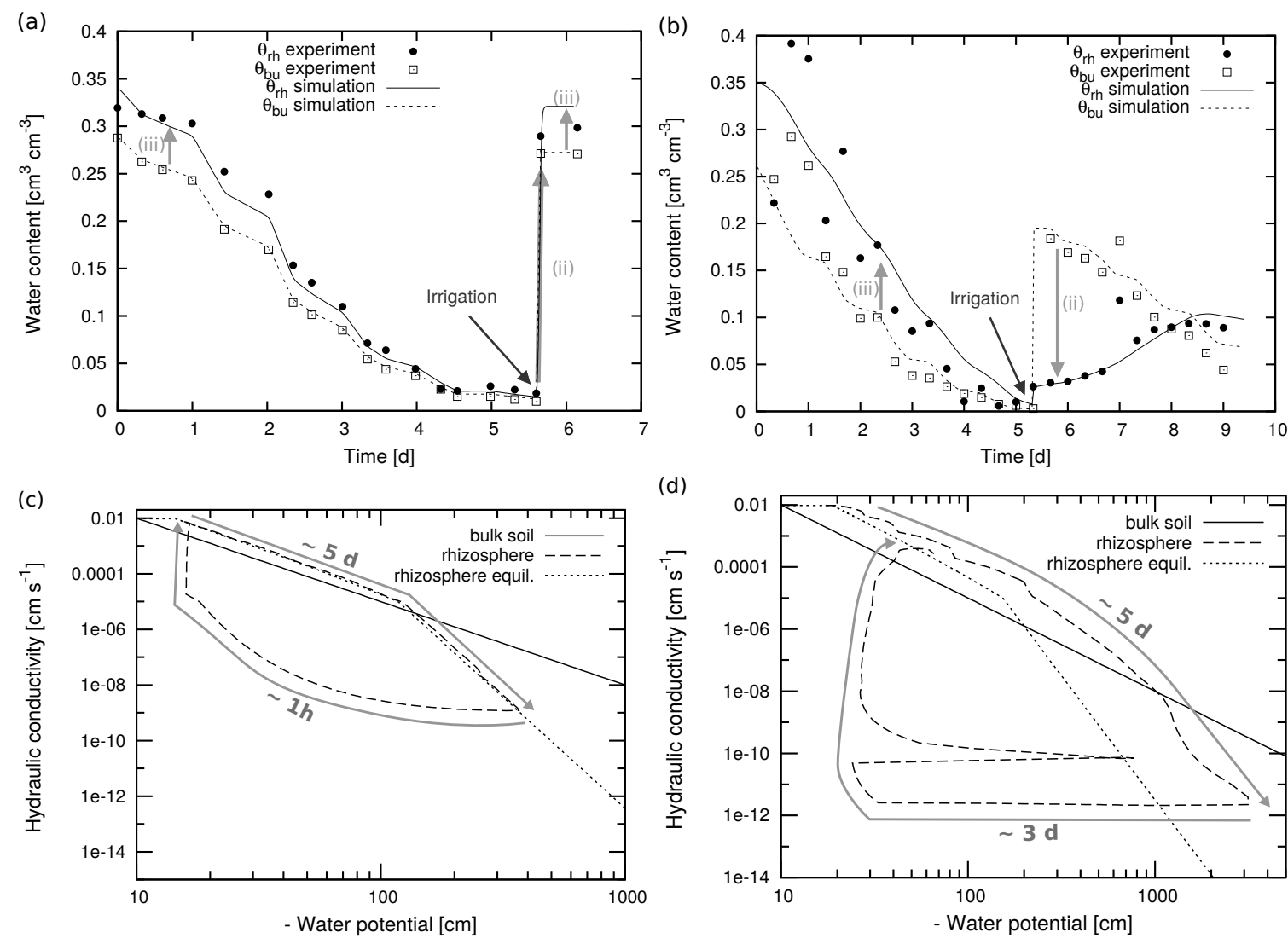

Figure 7.3: Top: evolution of average water content in rhizosphere and bulk soil during slow drying and subsequent rewetting for a young (a) and an old lupine root (b). Bottom: simulated hydraulic conductivity and equilibrium hydraulic conductivity for young (c) and old (d) rhizosphere as function of water potential. Also the bulk soil hydraulic conductivity is shown.

7.1). The assumption of rhizosphere hydraulic properties being the same as those of the bulk soil (classic) cannot produce these hysteretical dynamics. In particular, the widening of the curves between the rising and decreasing phases of the experiments cannot be preproduced by the classic Richards' equation. The measured data suggest an additional resistance in the rhizosphere which can also be reproduced by our model (parameter group (iv), Tab. 7.1).

By fitting the parameters to each of the three different runs D, E and F a slightly different parameter set has been obtained for each of them. These differences could be explained by the limitations of a single root model, i.e. in reality water might redistribute along the soil profile in the period between the runs in a way that cannot be captured by a one-dimensional model of root water uptake. Additionally the time steps between the measurements are not exactly known, which can lead to different conditions of the different runs.

In the experimental data it is well visible that, at equal transpiration rates, the suction 
(a)
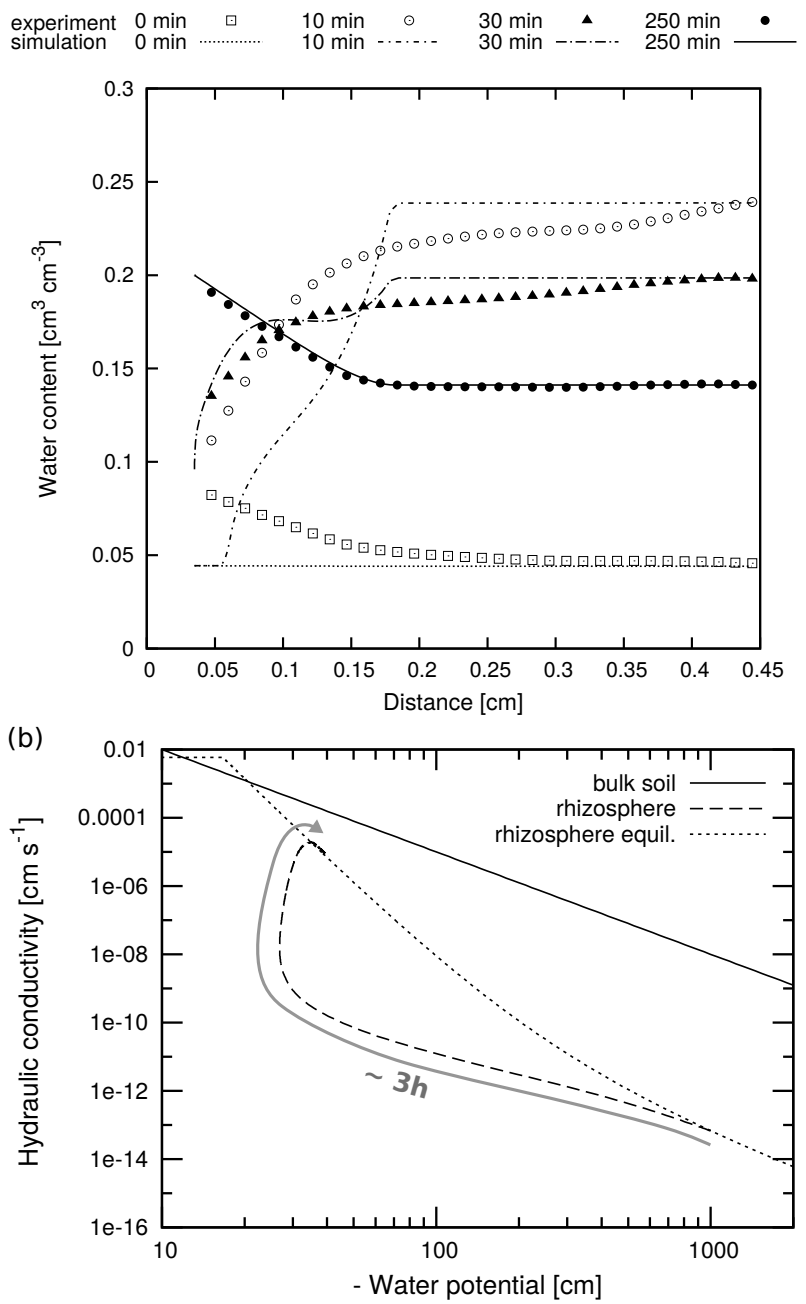

Figure 7.4: (a) Simulated and measured (Zarebanadkouki et al., 2015) evolution of water content as function of distance to the root surface of a lupine during drying and after irrigation. (b) Simulated hydraulic conductivity and equilibrium hydraulic conductivity of the rhizosphere at the root surface as function of water potential. Also the bulk soil hydraulic conductivity is shown. 

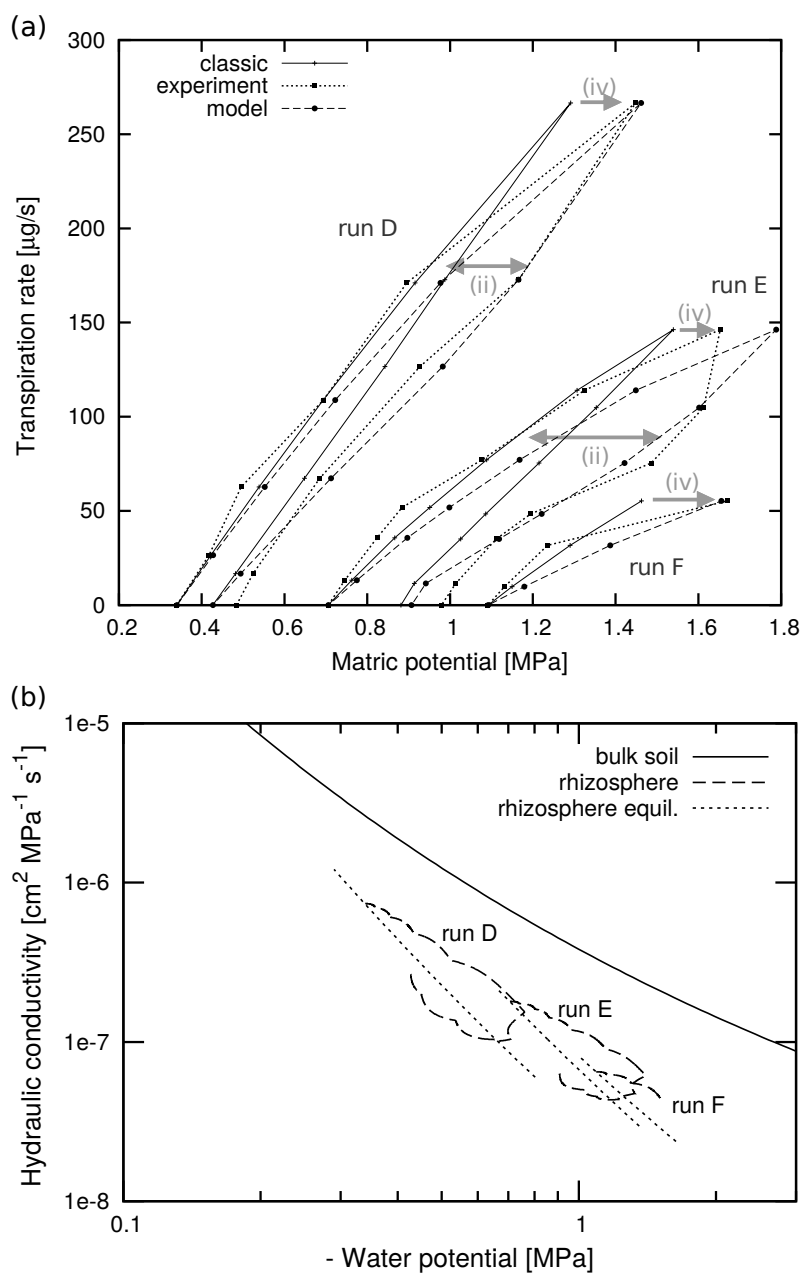

Figure 7.5: (a) Relation between water potential in the leaf xylem and transpiration rate. The dotted line shows the measured data taken from Passioura (1980), the continuous line shows the result of a simulation under the assumption that the rhizosphere has the same hydraulic properties as the bulk soil, and the dashed line shows the results of a simulation using our model. (b) Hydraulic conductivity as function of water potential. 


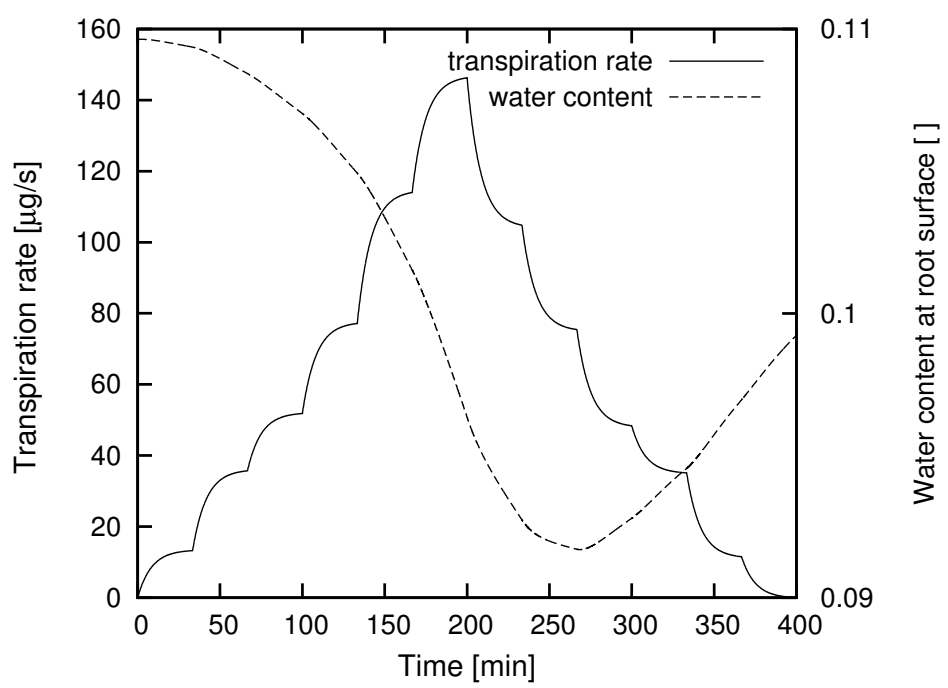

Figure 7.6: Transpiration rate and simulated water content in the rhizosphere at the root surface for run $\mathrm{E}$ of the experiment of Passioura (1980). The non-equilibrium concept in the rhizosphere means that at the time when transpiration is highest water content has not yet reached its lowest value.

in the leaf is much higher when the transpiration rate decreases than when it increases. Passioura (1980) and Deery et al. (2013) explained this observation with an additional resistance developing across the plant-soil continuum. Our model can explain and reproduce this additional resistance - here it is explained by the time-delay of water content change: when transpiration rate is highest and starts to decrease, the water content in the rhizosphere has not yet reached its equilibirum water content and hence the rhizosphere close to the root is still drying, leading to an increasing resistance at the root surface (Fig. 7.6). As a result, the necessary suction at the root surface increases while the transpiration rate already decreases.

\subsubsection{Summary of the results}

The set of parameters for each of the experiments is shown in (Tab. 7.1). Parameter $a$ describes the ratio of non-mucilage-filled pores $R$ as function of mucilage concentration. In the drying experiment $a$ is more than an order of magnitude smaller because of the different experimental set-up and the horizontal distribution of mucilage around the root Ahmed et al. 2014). The relaxation dynamics of mucilage (parameter group (ii), Tab. 7.1) depend on root age: for rewetting of old rhizosphere of a lupine $\tau_{0}$ is two orders of magnitude larger than for the rewetting of young rhizosphere of a lupine. For the experiment on wheat $\tau_{0}$ is even larger. Among the different experiments the parameters of group (iii) (Tab. 7.1), 
describing the equilibrium water retention curve, are of the same magnitude except for $\omega_{0}$ in the experiment with chia seed mucilage. We tested that a fit of the water retention curve of that experiment with $\beta=1$ results in a value for $\omega_{0}$ which is of same magnitude as in the other experiments. Parameter group (iv) (Tab. 7.1) describing the reduced hydraulic conductivity $\left(c_{0}, d_{0}, c_{1}\right.$ and $\left.d_{1}\right)$ are all of same magnitude.

\subsection{Discussion}

The simulations have demonstrated that the presented model can describe non-equilibrium processes in the rhizosphere that have been reported in the literature. The observed nonequilibrium processes in the rhizosphere were explained by the drying and wetting of mucilage. We applied a set of equations that can describe these time-dependent processes. The fitted parameters refer to the specific experiments and depend on several properties: mucilage composition, plant type, mucilage and root age, bacteria, history of water content, soil texture and pore size distribution. In particular, we found that the relaxation parameter $\tau_{0}$ increases with the age of the rhizosphere.

In Deery et al. (2013), an additional resistance within the root-soil continuum was introduced to reproduce the relation between transpiration and balancing pressure during the falling phase of the experiment. Our model is capable of describing the observed relation between transpiration and xylem pressure without introducing a new resistance, but simply by including the dynamic effects of mucilage on the hydraulic conductivity: the increase in equilibrium water content at constant water potential together with the reduction of soil hydraulic conductivity at same water content, results in a function for the equilibrium rhizosphere hydraulic conductivity which - depending on the mucilage concentration - might increase or decrease the bulk soil hydraulic conductivity at a negative water potential. Under non-equilibrium conditions, e.g. after exudation and irrigation, rhizosphere hydraulic conductivity can differ some order of magnitude from bulk soil hydraulic conductivity - typically it relatively increases during drying and it decreases during wetting.

The diffusion process of mucilage within the pore space certainly depends on water content, but in this work the focus has been on the water flow while the concentration of mucilage has been assumed to be constant during the observed process. An open question is how far and how quickly mucilage penetrates into the soil upon exudation. Another question is how roots and root exudates modify mechanical properties of soil, e.g. Aravena et al. (2010) and Aravena et al. (2013) showed compaction of rhizosphere caused by root growth. 
In the proposed model the functions have been kept as simple as possible under observance of reasonable assumptions and the requirement to reproduce the experimental data. Swelling of gel within the pore space of porous media together with the hydraulic problem is a complex process and it is not clear to what extent such a simplified model can be generalized. To discuss this question further experiments as well as numerical simulations are needed. A future application of this model is the implementation into three-dimensional architectural models of root water uptake, such as that of Javaux et al. (2008) or Dunbabin et al. (2013) or to consider rhizosphere hydraulic properties in models of nutrient uptake such as Leitner et al. (2010).

The hygroscopic properties of mucilage and the consequent high water holding capacity of the rhizosphere favours water availability to plants. Facilitation in water uptake does not result only from the increased water content of the rhizosphere at equilibrium conditions. The fact that mucilage dries more slowly than the bulk soil - i.e. non-equilibrium dynamics - results in the rhizosphere remaining wetter than what expected at equilibrium. It follows that during periods of increasing transpiration, as in Passioura's experiment, the lowest water content in the rhizosphere is not reached at the transpiration peak, but afterwords. In this way, at the transpiration peak, the rhizosphere is slightly wetter and might be more conductive compared to the hypothetical case without mucilage.

Hydrophobicity of dry mucilage may locally reduce the uptake of water after a drying/wetting cycle (Zarebanadkouki and Carminati, 2014), but it might also help plants to adapt to soil drying. For instance, when the top soil is dry and the sub soil is wet, then roots can take up water in the sub soil and the hydrophobic rhizosphere of the upper roots can prevent that water is sucked out of the roots into the dry soil. In conclusion, both characteristics of mucilage and their temporal dynamics have the potential to favour water availability to plants in dry conditions. 


\subsection{Supplementary Material}

Drying of fresh mucilage For details of the simulation of this experiment we refer to Ahmed et al. (2014).

Rewetting of dry young/old rhizosphere Lupines were grown in aluminum containers filled with as a sandy soil. During growth period, the plants were irrigated by capillary raise from bottom. When the plants were three weeks old the irrigation was stopped. During the following five days the plant took up water from the soil and the water content decreased. To minimize evaporation, the upper side of the containers were covered with aluminium tape. On day six the plants started to show severe wilting symptoms and the water reservoir was re-connected to the containers allowing capillary rise at a water potential of $h=-20 \mathrm{~cm}$ at the bottom of the container. In this way the soil rewetted and the plants were able to recover. In the following days water content of the samples decreased again as plants took up water from soil. During drying and subsequent rewetting cycles the water redistribution in the soil was monitored using a time series neutron radiography at three times a day with an interval of 8 hours. Data of neutron radiography are presented in Carminati et al. (2010). After root segmentation, the average soil water content in the bulk soil and the rhizosphere was quantified through the radiographs. Based on the profile of soil water content around the roots during drying and rewetting cycle, they defined the rhizosphere as a region with distance of around $2 \mathrm{~mm}$ from root surface. They observed that during the first drying cycle water content in the rhizosphere was higher than in the bulk soil. After re-wetting, the water content of the bulk soil quickly recovered, while the rhizosphere stayed temporarily drier than the bulk soil and it took almost two days until the water content recovered in the rhizosphere of the old plant. The authors concluded that the rhizosphere had different hydraulic properties than the bulk soil. Fig. 7 from Carminati et al. (2010) shows that the region of distinct hydraulic properties has a characteristic extension of $0.15-0.2 \mathrm{~cm}$.

As hydraulic parameters of the bulk soil we used the values measured by Carminati et al. (2010) (Tab. 7.2). As suggested in Carminati et al. (2010) the radius of the root for the single-root model is: $r_{\text {root }}=0.05 \mathrm{~cm}$, the outer radius of the rhizosphere: $r_{r h i z}=0.2 \mathrm{~cm}$ and the radius of the bulk soil: $r_{b u l k}=1 \mathrm{~cm}$. As boundary condition at $r_{\text {root }}$ we used the flux $[\mathrm{cm} / \mathrm{s}]$ that is related to root water uptake: the measured differences in weight of the sample was divided by root surface, density of water, and the time intervals between measurements. As a result of this procedure a day and night cycle of the root water uptake is still visible 
Table 7.2: Hydraulic parameters used for the simulation. The parameters have been taken from Carminati (2012). To account for the low bulk soil water contents measured with neutron radiography, the residual water content has been modified from 0.01 to 0.00

\begin{tabular}{cccccc}
\hline$\theta_{s}$ & $\theta_{r}$ & $\lambda$ & $h_{0}[\mathrm{~cm}]$ & $\tau$ & $K_{\text {sat }}[\mathrm{cm} / \mathrm{s}]$ \\
\hline 0.45 & 0.00 & 1 & -10 & 3 & $10^{-2}$ \\
\hline
\end{tabular}

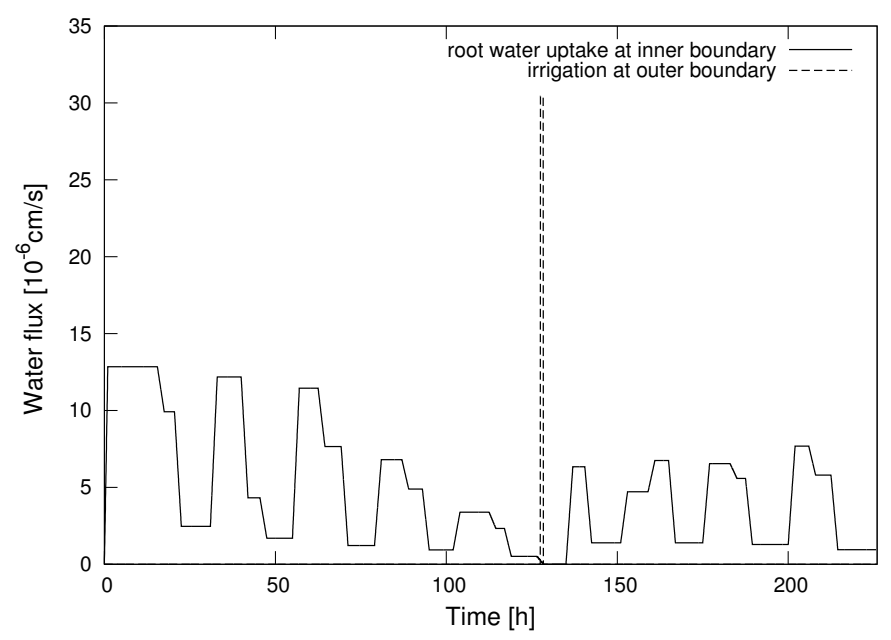

Figure 7.7: Boundary flux used for simulating the experiment described in Carminati (2012). It is obtained from the data of the weight of the sample, see Fig. 2 in Carminati (2012).

(Fig. 7.7). However, by averaging the flow over the entire time interval between the weight measurements, daily high and low transpiration peaks were dumped.

The described procedure lead to an inflow during the time-interval where re-wetting took place. This inflow has been used as outer boundary condition - the value has been multiplied by the ratio of inner and outer boundary - and the boundary condition at the root surface was chosen as no-flow. For the remaining time intervals, we imposed no-flow at the outer boundary (Fig. 7.7).

The hydraulic properties describing the non-equilbrium of the rhizosphere have been hand-fitted so that they matched well with the measured water content dynamics in bulk soil and in rhizosphere.

Water content distribution during rewetting of dry rhizosphere The experimental conditions are very similar to the above described experiment. Therefore in the numerical simulation the bulk soil parameters are same as before. Since the experiment was during night, there were no-flow conditions at the root surface. The boundary conditions at the bulk soil were extracted from the measured data. $r_{\text {rhiz }}=0.16 \mathrm{~cm}$ was fitted to the measured 
Table 7.3: Parameter set for the hydraulic properties of the bulk soil used by Passioura (1980). The parameters have been obtained by least-square fitting to the data points of water retention and conductivity (Fig. 7.8).

\begin{tabular}{ccccc}
\hline$\theta_{s}$ & $\lambda$ & $\psi_{e}[\mathrm{MPa}]$ & $b$ & $K_{0}\left[\mathrm{~cm}^{2} \mathrm{MPa}^{-1} \mathrm{~s}^{-1}\right]$ \\
\hline 0.2 & 0.263 & -0.0486 & 13.01 & $1.075 \mathrm{e}-9$ \\
\hline
\end{tabular}

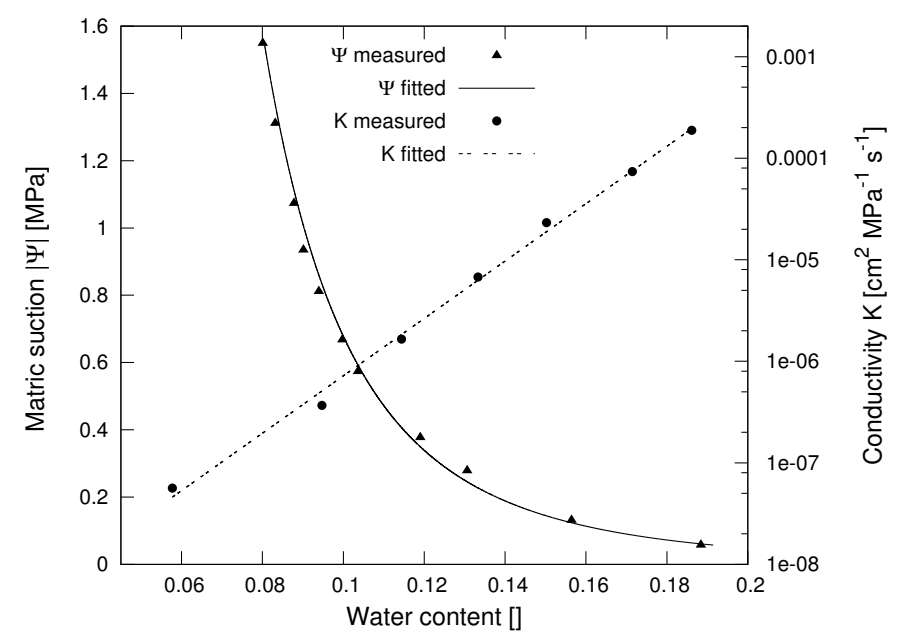

Figure 7.8: Hydraulic properties of the bulk soil used in Passioura (1980). The points show the measured values taken from Passioura (1980) and the lines are the fitted hydraulic functions.

data as well.

Wheat xylem pressure during increasing and decreasing transpiration The hydraulic properties for the bulk soil (see Fig. 7.8 and Tab. 7.3) have been fitted to the data measured by Passioura (1980).

For the water retention curve, we chose Campbell's model (Campbell, 1974):

$$
\begin{aligned}
& \theta=\theta_{s}\left[\frac{\psi}{\psi_{e}}\right]^{-\lambda} \quad \text { if } \psi<\psi_{e} \\
& \theta=\theta_{s} \quad \text { if } \psi \geq \psi_{e}
\end{aligned}
$$

where $\theta_{s}$ and $\psi_{e}$ are the saturated water content and the air entry pressure of the bulk soil. The parameters in Tab. 7.3 were estimated using a least squares fit. Passioura (1980) measured the hydraulic diffusivity at several water contents. Based on the water retention curve (Eqn. (7.15) ) and the measured data points of diffusivity, the hydraulic conductivity is calculated as: 


$$
K\left(\theta_{i}\right)=\left.D\left(\theta_{i}\right) \frac{\partial \theta}{\partial \psi}\right|_{\psi=\psi\left(\theta_{i}\right)}
$$

The obtained values for the hydraulic conductivity are shown in Fig. 7.8. An exponential function seemed to fit very well the relation between hydraulic conductivity and water content:

$$
K(\theta)=K_{0} \exp \left(b \frac{\theta}{\theta_{s}}\right)
$$

The fitting parameters are reported in Tab. 7.3 .

The soil volume was $V=294.5 \mathrm{~cm}^{3}$. Passioura (1980) suggested that the root length per unit volume that is active in water uptake is around $1.4 \mathrm{~cm} \mathrm{~cm}^{-3}$, which is circa $30 \%$ of the total root length per unit volume, $5.2 \mathrm{~cm} \mathrm{~cm}^{-3}$. The total length of active roots in the pot is $l=412.3 \mathrm{~cm}$. The mean root radius was $r_{\text {root }}=0.015 \mathrm{~cm}$ which leads to a total active root surface of $A=2 \pi l r_{\text {root }}=38.86 \mathrm{~cm}^{2}$.

The average water flux at the root surface $j\left[\mathrm{~cm} \mathrm{~s}^{-1}\right]$ can be obtained by dividing the measured transpiration rate $E\left[\mu \mathrm{g} \mathrm{s}^{-1}\right]$ by the active root surface $A$ and the density of water at room temperature:

$$
j=2.578 \mathrm{e}-8{\mathrm{~cm} \mu \mathrm{g}^{-1} \cdot E}^{-1}
$$

The total volume of the pot and the total length of active roots lead to the outer radius of the cylinder:

$$
r_{c y l}=\sqrt{\frac{V}{\pi l}}=0.4768 \mathrm{~cm}
$$

The hydraulic resistance $R_{\text {plant }}$ of the plant (from root surface to leaf xylem potential) is assumed to be constant. Then the pressure drop between root surface and leaf xylem is: $\Delta \psi_{\text {plant }}=E \cdot R_{\text {plant }}$. Under saturated soil conditions the resistance of the soil can be neglected which means that $\Delta \psi_{\text {tot }}=\Delta \psi_{\text {plant }}$, where $\Delta \psi_{\text {tot }}$ is the total difference in water potential between soil and leaf xylem. Indeed Passioura observed a linear relation between $\Delta \psi_{\text {tot }}$ and $E$ under saturated soil conditions as can be seen in the first cycle of Fig. 3 in Passioura (1980)). By calculating the slope of this line one obtains the resistance of the plant:

$$
R_{\text {plant }}=2.632 \mathrm{e}-3 \mathrm{MPa} \mathrm{s} \mu \mathrm{g}^{-1}
$$

Eq. 7.20 leads to the water potential at the root surface in this one-dimensional singleroot model: 


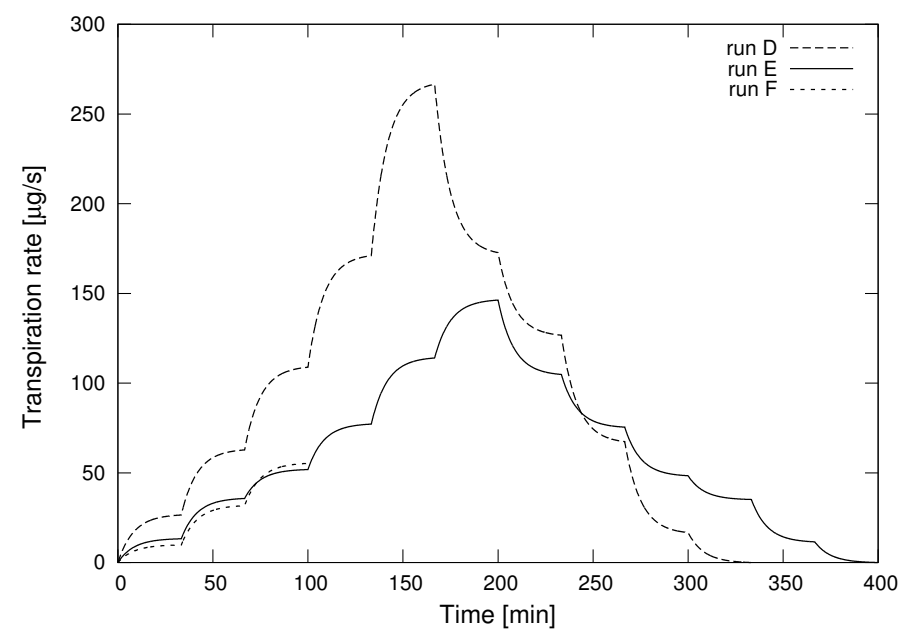

Figure 7.9: Transpiration rate versus time for the simulation of the experiment of Passioura (1980). It has been assumed that the transpiration rate increases in each step exponentially to the new transpiration rate and that the time interval of each step is 2000 s.

$$
\psi_{\text {root }}=\psi_{\text {leaf }}+R E
$$

where $\psi_{\text {leaf }}$ is the water potential in the leaf xylem.

In the simulation, no-flow was imposed as outer boundary condition at $r=r_{b u l k}$, and $j$ as inner boundary condition at $r=r_{\text {root }}$. The parameters used for the above described model of the hydraulic non-equilibrium in the rhizosphere have been fitted by hand in such a way that a good correlation is achieved between simulated and measured water potential at the root surface. 


\section{Conclusions}

\subsection{Summary}

Water flow from soil into roots strongly depends on hydraulic dynamics in the rhizosphere. Although it is very thin, all the water taken up by roots has to flow across this layer. Its hydraulic conductivity is controlled by complex bio-physical processes involving mucilage and soil.

We developed a model describing the rewetting of dry rhizosphere. We found that below a certain concentration of mucilage water can easily cross the rhizosphere, at higher concentrations of mucilage water could no longer cross it during the first minutes or even hours after irrigation. The mucilage concentration at percolation threshold depends on mean soil particle size: the smaller the particles, the bigger the soil specific surface and the more mucilage is needed to make the soil surface hydrophobic and to prevent water flow across the thin layer.

We found that the threshold depends also on bulk soil water potential after irrigation: the higher the water potential the more mucilage is needed to prevent water flow across the layer. Combining a pore-network model with the Young-Laplace equation gives an estimation of mucilage concentration at percolation threshold as function of water potential and mean soil particle size (Eq. 4.10.

The percolation model as well as our experiments (Fig. 2.5p) showed, that for mucilage concentrations slightly below the percolation threshold water could flow across the layer without rewetting it significantly. When describing water flow on the larger scale using the Richards equation a non-equilibrium relation between water content and water potential needs to be included to describe these dynamics: while changes of water potential can be transported across this layer within a short time it can take up to two days for the water content to recover within the layer.

We presented a model for the hydraulic dynamics of rhizosphere motivated by physical properties of gel-soil mixtures: (a) we included the non-equilibrium relation between water potential and water content, (b) we modified the water retention curve by adding a term similar to an osmotic potential to account for the water holding capacity of mucilage and (c) the hydraulic conductivity has been scaled by a factor accounting for the higher viscosity of mucilage which reduces the mobility of water molecules. The model was tested on various experiments from the literature. 


\subsection{Application, limitations and outlook}

Our studies, together with former observations of water dynamics in the rhizosphere, suggest that under some conditions the rhizosphere is near the percolation threshold, where small variations in mucilage concentration sensitively control the rhizosphere hydraulic conductivity. Is mucilage exudation a plant mechanism to efficiently control the rhizosphere conductivity and the access to water?

We mentioned that the relaxation of mucilage depends on root and mucilage age: after irrigation water content of young rhizosphere recovers quickly while it takes up to two days for the water content of old rhizosphere to recover. How does the relaxation time and other parameters of our model depend on bio-chemical processes that alter the mucilage? Bacteria, for example, consume mucilage but also produce mucilage.

We kept the functions of the model for the rhizosphere hydraulic properties as simple as possible under observance of reasonable assumptions and the requirement to reproduce the experimental results. However, swelling of gel within the pore space coupled with the hydraulic problem of water flow is a complex system and it is not clear if such a simplified model can be generalized. To discuss this question further numerical simulations as well as experiments are needed. A possible future application of this model is the implementation into complex three-dimensional architectural models of root water uptake, such as that of Javaux et al. (2008).

Major challenges to experimentally determine the rhizosphere hydraulic conductivity are the small extension of the layer as well as its long equilibration time. Especially under dry conditions huge gradients can develop across this layer which has an extension of just a few millimeters. These gradients make it tricky to measure the local water potential with tensiometers. Under saturated conditions mucilage reduces the hydraulic conductivity. It would be exciting to experimentally measure if mucilage increases the hydraulic conductivity at low water potentials close to the wilting point. This, however, could be a tricky task since it takes a very long time to bring soil-mucilage-mixtures at these low water potentials to local equilibrium conditions.

The fact that mucilage dries more slowly than the bulk soil - i.e. non-equilibrium dynamics - results in the rhizosphere remaining wetter than what expected at equilibrium. It follows that during periods of increasing transpiration the lowest water content in the rhizosphere is not reached at the transpiration peak, but afterwords. In this way, at the transpiration peak, the rhizosphere is slightly wetter and might be more conductive compared to the hypothetical 
case without mucilage.

Locally hydrophobicity of dry mucilage may reduce the uptake of water after a drying/wetting cycle (Zarebanadkouki and Carminati, 2014), but it might also help plants: for instance, when the top soil is dry and the sub soil is wet, then roots can take up water from the sub soil and the hydrophobic rhizosphere of the upper roots can prevent that water is sucked out of the roots into the dry soil. In conclusion, both characteristics of mucilage and their temporal dynamics have the potential to favour water availability to plants in dry conditions.

In this thesis we focused on the impact of mucilage on water flow dynamics, only. However, water content itself will also influence diffusion and distribution of mucilage in soil. An open question is how far and how quickly mucilage penetrates into the soil upon exudation and how this depends on soil hydraulic conditions. Independent measurements of mucilage distribution in the rhizosphere would help to fully understand the complex interactions between water and mucilage in porous media. Finally, interdisciplinary research in both, plant and soil science is needed to understand the implications of such biophysical processes in the rhizosphere for plant water relations. 


\title{
A Numerical simulation of coupled heat, liquid water and wa- ter vapor in soils for heat dissipation of underground elec- trical power cables
}

written by Eva Kroener, Andrea Vallati and Marco Bittelli, published in Applied Thermal Engineering (Kroener et al., 2014a)

\begin{abstract}
The trend towards renewable energy comes along with a more and more decentralized production of electric energy. As a consequence many countries will have to build hundreds or even thousands of miles of underground transmission lines during the next years. The lifetime of a transmission line system strongly depends on its temperature. Therefore an accurate calculation of the cable temperature is essential for estimating and optimizing the systems lifetime.

The International Electrotechnical Commission and the Institute of Electronics and Electrical Engineers are still employing classic approaches, dating back from the 1950s, that are missing fundamental phenomena involved in heat transport in soils. In recent years several authors (Saito et al. 2006, Bittelli et al., 2008) pointed out that for a proper computation of heat transport in soils, physical processes describing heat, liquid water and vapor transport must be coupled and the respective environmental weather conditions need to be considered.

In this study we present a numerical model of coupled liquid water, vapor and heat flow, to describe heat dissipation from underground cables. At first the model is tested and validated on a downscaled experiment (Minopoli, 2007/2008), secondly the model is applied on a simplified system to demonstrate the strong relation of the cables temperature on soil water content and finally the model is applied using real weather conditions to demonstrate that small changes in the design of underground transmission line systems can lead to considerable improvements in both average as well as peak-to-peak temperatures.
\end{abstract}

\section{A.1 Introduction}

In the past the high prices and operational limitations of laying power cables subterraneously was one of the major reasons for using overhead lines. In the last years the need to connect an increasing number of new wind farms, is forcing many countries to face the prospect of installing hundreds of miles of new cables and hundreds more pylons across the countryside. 
Therefore the use of underground power cables has become now the only valuable alternative.

Increasing their lifetime is one option of reducing the high costs of underground transmission line systems. Ageing of underground power cables (Densley, 2001) can be accelerated by thermal (expansion/contraction, melting/flow of insulation, chemical reaction), electrical (electrical/water treeing, intrinsic breakdown), mechanical (yielding, cracking, rupture) and environmental factors (corrosion). These ageing mechanisms depend strongly on temperature: in the $80-110^{\circ} \mathrm{C}$ range the degradation rate doubles with an increase of $8-10^{\circ} \mathrm{C}$ (Cigre, 1998). An experimental study (Chen et al., 2012) demonstrated that the mechanism of electrical treeing is very sensitive to temperature: the average growth rate can be more than 100 times faster at $70^{\circ} \mathrm{C}$ than at $10^{\circ} \mathrm{C}$.

Hence a correct design of a buried underground power cable is based on its thermal analysis, needed to determine the ampacity of the cable, i.e., its current capacity. The detailed calculations employed to design an underground cable system have been developed many years ago: The International Electro-technical Commission (IEC) and the Institute of Electronics and Electrical Engineers (IEEE) employ the classic approach of Neher and McGrath (Oct. 1957). This approach is based on the assumption that the soil is homogeneous and the thermal conductivity is constant. Clearly, these assumptions are not realistic, and although the soil used to fill the trench may have homogeneous properties, the soil surrounding the backfill has different properties. Because of the limitations of the classic approach, several analytical (Papagiannopoulos et al., 2013, Chatziathanasiou et al., 2013; de Lieto Vollaro et al. 2011b) and numerical (Kovac et al., 2013, Canova et al., 2012, de Lieto Vollaro et al., 2011a; De Leon and Anders, 2008) studies have been proposed.

However, these studies are still missing some fundamental physical aspects of heat dissipation in natural soils. Since early works in the 1950's and 1960's (Taylor and Cavazza, 1954; de Vries, 1963), it was found that the movement of soil heat, water vapor, and liquid water in soils are coupled. A very important process that determines the coupling between water and heat, is the transport of latent heat by vapor flux within the soil. Latent heat transport is not only related to changes in humidity but in non-isothermal processes it is also driven by temperature gradients. Soil temperature may be significantly underestimated when the energy transfer associated with vapor is not considered (Saito et al., 2006).

On the other hand, infiltration fronts after heavy rainfall events cause strong convective transfer of thermal energy away from the cable and can lead to sudden cooling of the cable. Sudden changes of temperature lead to shrinking and following expansion of the cable. These 
mechanical stresses within the system can favor electric treeing and shorten the systems lifetime.

Another important issue for a correct computation is the estimation of soil thermal conductivity. The seminal study of de Vries (1963) demonstrated that soil thermal conductivity is dependent on the soil textural composition. Moreover, Campbell et al. (1994) showed that soil thermal conductivity increased dramatically with temperature in moist soils. These results are a clear indication that thermal conductivity cannot be assumed to be a constant parameter and that knowledge of soil water content is necessary for a correct estimation of thermal conductivity.

Overall, to correctly describe heat dissipation of buried underground cables it is necessary to simulate coupled heat, liquid water and vapor fluxes for non-homogeneous materials and to include in the computation the dependence of thermal conductivity on soil properties and water content.

In this paper, we present a two-dimensional numerical model for computation of coupled heat, liquid water and vapour fluxes of an underground power transmission system. The numerical model will be applied:

1. to a downscaled experiment to validate the model,

2. to a system with realistic dimensions, but simplified geometry in order to highlight the importance of considering water flow when discussing heat dissipation from an underground cable,

3. to a system with realistic dimensions and geometry under consideration of real weather conditions during a 300 day-period.

Aim of this study is to demonstrate the key role of water flow - in particular of weather and soil conditions - in the overall energy budget and to present and validate a numerical model for heat dissipation from an electrical power cable under consideration of hydraulic dynamics. In this way we present an approach that opens new possibilities to optimize the design of underground transmission line systems under the constraints of the region's weather and soil conditions. 


\section{A.2 Theory}

\section{A.2.1 Description of coupled model}

The following description follows mainly Bittelli et al. (2008) where a one dimensional model of coupled heat, water and vapor flow has been introduced and validated with an evaporation experiment from bare soil under real weather conditions. Here we will provide a short description of the equations employed. More detailed informations about the parametrizations of soil hydraulic properties and interactions between soil and atmosphere can also be found in Campbell (1985).

Transport equations Liquid water flow is described by Richards' equation, where water flow $q_{w}$ is driven by a gradient in water potential (sum of matric potential $\psi$ and gravitational potential $g z$ ) and is proportional to the hydraulic conductivity $K$ :

$$
q_{w}=-K \nabla(\psi+g z)
$$

$K$ depends in a highly non-linear way on the matric potential $\psi$.

According to Fick's law water vapor flow is driven by a gradient in water vapor concentration. Vapor concentration depends on both humidity $h$ and temperature $T$. Therefore the total water vapor flow $q_{v}$ can be formulated as sum of an isothermal flux component $q_{v, i}$ and a temperature driven flux component $q_{v, T}$ :

$$
q_{v}=q_{v, i}+q_{v, T}=-D_{v} c_{v}^{\prime} \nabla h-D_{v} h s \nabla T
$$

where $D_{v}$ is vapor diffusivity, $c_{v}^{\prime}$ saturated vapor concentration, $s$ the slope of saturation vapor concentration function and humidity $h=c_{v} / c_{v}^{\prime}$ is the fraction between vapor concentration $c_{v}$ and saturated vapor concentration $c_{v}^{\prime}$.

Thermal energy flow in soils can be divided into sensible, latent heat and the convective part carried by flowing liquid water. Sensible heat flow is driven by a gradient in temperature $T$ and is proportional to the thermal conductivity $\lambda$. Latent heat flow is the thermal energy carried by water vapor. It is proportional to the sum of latent heat of vaporization $L$ and thermal energy of liquid water $T C_{w}$. Thermal energy carried by liquid water is proportional to water flow $q_{w}$ and thermal energy of water $T C_{w}$. Therefore the total heat flow $q_{h}$ is:

$$
q_{h}=-\lambda \nabla T+\left(L+T C_{w}\right) q_{v}+T C_{w} q_{w}
$$

Conservation of mass provides the relation between flow of water, both in liquid as well as vapor form, and change of water content $\rho_{w} \theta$, where $\rho_{w}$ is the density of water and $\theta$ the 
volumetric water content:

$$
\rho_{w} \frac{\partial \theta}{\partial t}=-\nabla \cdot\left(q_{w}+q_{v}\right)
$$

Under low water potentials, the hydraulic conductivity of liquid water becomes very small and therefore the contribution of vapor flow can then be in the same range as liquid water flow or even bigger making vapor flow a key element of water transport. The contribution of water vapor to the local water content, however, can be neglected.

Conservation of energy states that change in specific heat equals to negative divergence of thermal energy flow. Specific heat is stored in the liquid water, $C_{w} \rho_{w} \theta$, the solid part, $C_{m} \rho_{m} x_{m}$ and the air fraction. $\rho_{m}$ is the specific density of minerals, $x_{m}$ the volumetric fraction of the solid part, $C_{m}$ is the specific heat of soil minerals and $C_{w}$ the specific heat of water. Changes in the thermal energy stored in the air fraction, however, are so small compared to changes in thermal energy in the liquid and solid fraction that we can neglect the contribution of the air fraction to the changes in thermal energy:

$$
\left[C_{m} \rho_{m} x_{m}+C_{w} \rho_{w} \theta\right] \frac{\partial T}{\partial t}=-\nabla \cdot q_{h}
$$

\section{Parameter functions}

Hydraulic parameters Campbell's parametrization (Campbell and Shiozawa, 1992) is used to describe the relation between water content and water potential and between hydraulic conductivity and water potential:

$$
\begin{gathered}
\theta= \begin{cases}\theta_{s}\left(\frac{\psi_{e}}{\psi}\right)^{1 / b} & \text { if }\left(\psi<\psi_{e}\right) \\
\theta_{s} & \text { if }\left(\psi \geq \psi_{e}\right)\end{cases} \\
K= \begin{cases}K_{s}\left(\frac{\psi_{e}}{\psi}\right)^{2+3 / b} & \text { if }\left(\psi<\psi_{e}\right) \\
K_{s} & \text { if }\left(\psi \geq \psi_{e}\right)\end{cases}
\end{gathered}
$$

where $\psi_{e}$ is air entry potential, $\theta_{s}$ saturated volumetric water content, $b$ a shape parameter, $K$ unsaturated hydraulic conductivity and $K_{s}$ is saturated hydraulic conductivity. Saturated water content $\theta_{s}$ is equal to the porosity $\Phi$ and is related to bulk density $\rho_{b}$ and average density of soil minerals $\rho_{m}$ :

$$
\Phi=1-\frac{\rho_{b}}{\rho_{m}}
$$

Water retention curve and hydraulic conductivity function for a sandy soil and for expanded clay aggregates - these materials have been used by Minopoli $(2007 / 2008)$ - can be seen in 

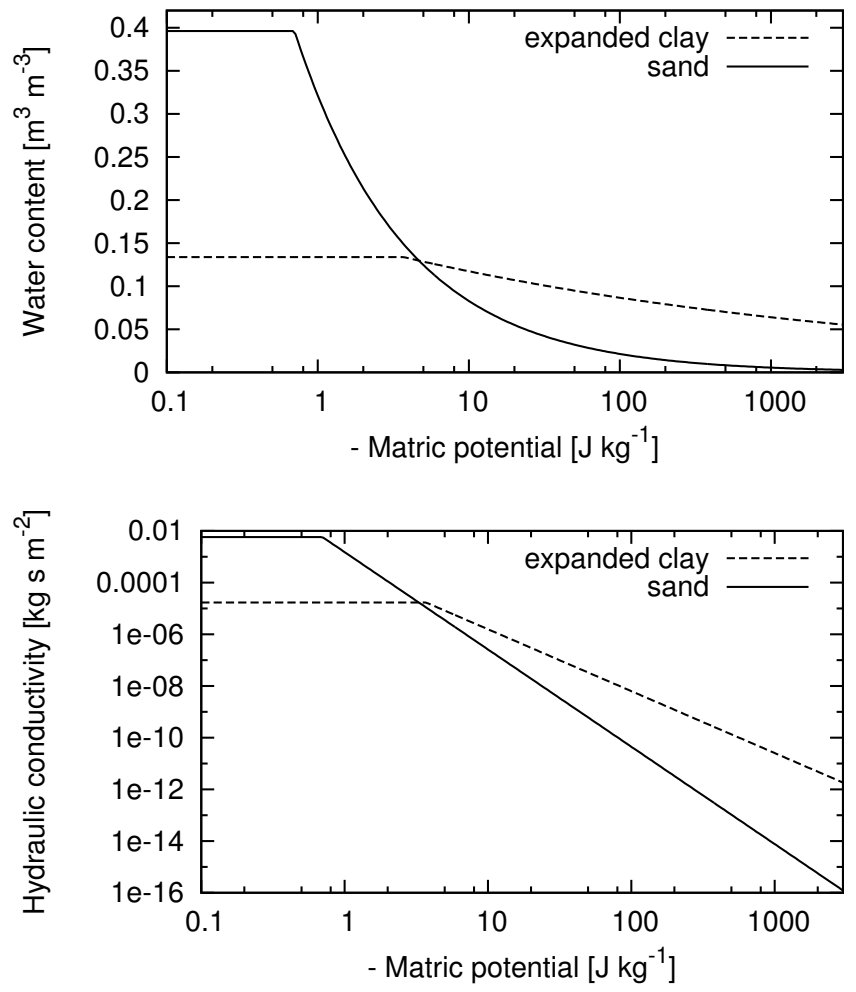

Figure A.1: Water retention curve and hydraulic conductivity function for two materials that have been used in the simulations, see table A.1. 
Fig A.1. While this parametrization works fine for many soil types, the hydraulic properties of expanded clay aggregates are very complex because they have on one hand very large pores between their aggregates and on the other hand very small pores within their aggregates. The large pores drain very fast, while the small pores drain in the same way as normal clay soils. In this study the water potential is negative enough that the big pores are always drained and changes in total water content are caused by processes within the aggregates. These processes can be described by typical clay hydraulic parameters. However, to account for the drained big pores the saturated water content is reduced by multiplying it with the fraction between measured density of the used expanded clay aggregates to an average bulk density of soil: $\theta_{s}($ clay ag $)=\theta_{s}$ (clay) $\frac{\left.\rho_{b} \text { (clay ag }\right)}{\rho_{b}}$. While there exist much more elaborated concepts for dual porosity water flow (Gerke and Genuchten, 1993 , Larsson and Jarvis, 1999) the simulations of this study are not very sensitive on the liquid water flow in the region of clay aggregates and hence the above described simplified model should be sufficient here.

Vapor diffusion parameters Humidity is related to water potential:

$$
h=\exp \left(\frac{M_{w} \psi}{R T_{K}}\right)
$$

where $M_{w}$ is the molecular weight of water, $T_{K}$ the Kelvin temperature and $R$ the gas constant.

The slope of saturation vapor concentration function is: $s=\Delta M_{w} /\left(R T_{K}\right)$ where $\Delta$ is the slope of the saturation vapor pressure curve. Multiplication by $M_{w} /\left(R T_{K}\right)$ is necessary to convert pressure $[\mathrm{kPa}]$ to concentration $\left[\mathrm{kg} / \mathrm{m}^{3}\right]$. For the vapor pressure curve we used the equation of Buck (1981):

$$
e_{s}(T)=0.611 \exp \left(\frac{17.27 T}{T+237.3}\right)
$$

where $T$ is temperature in $\left[{ }^{\circ} \mathrm{C}\right]$. Evaluating the derivative with respect to temperature yields the slope of vapor pressure curve:

$$
\Delta=\frac{4098 \cdot e_{s}}{(T+237.3)^{2}}
$$

Saturation vapor concentration is: $c_{v}^{\prime}=e_{s} M_{w} /\left(R T_{K}\right)$.

Water vapor diffusivity $D_{v}$ depends on the air filled porosity and on the binary diffusion coefficient of water vapor in air $D_{0}(T, P)$ :

$$
D_{v}=D_{0}(T, P) \epsilon\left(x_{a}\right)
$$


where $\epsilon$ is a parameter that depends on the air filled volume fraction $x_{a}$ :

$$
\epsilon\left(x_{a}\right)=\beta\left(x_{a}\right)^{m}
$$

with $\beta=0.9$ and $m=2.3$ (Penman, 1940). The binary diffusion coefficient of water vapor in air is:

$$
D_{0}\left(T_{K}, P\right)=D_{0}(273.15 \mathrm{~K}, 101.3 \mathrm{kPa})\left(\frac{T_{K}}{273.15 \mathrm{~K}}\right)^{1.75}\left(\frac{101.3 \mathrm{kPa}}{P}\right)
$$

with the binary diffusion coefficient for standard conditions: $D_{0}(273.15 \mathrm{~K}, 101.3 \mathrm{kPa})=$ $2.12 \times 10^{-5} \mathrm{~m}^{2} \mathrm{~s}^{-1}$.

Parameters for heat flow The thermal conductivity $\lambda$ of soil strongly depends on both mineral as well as water content and additionally on the soil's temperature. In our simulations the apparent thermal conductivity model from Campbell et al. (1994) - a model that is based on the theory of de Vries (1963) - is used. They fitted their model to thermal conductivity measurements of soil samples differing in texture, bulk density, water content and temperature. In their model the thermal conductivity is a weighted average of the components' conductivities, water $\lambda_{w}$, air $\lambda_{a}$ and minerals $\lambda_{m}$ :

$$
\lambda=-\frac{k_{w} \theta \lambda_{w}+k_{a} x_{a} \lambda_{a}+k_{m} x_{m} \lambda_{m}}{k_{w} \theta+k_{a} x_{a}+k_{m} x_{m}}
$$

The conductivities are weighted by the components' volume fractions $\left(\theta, x_{a}\right.$ and $\left.x_{m}\right)$ and by additional weighting factors $\left(k_{w}, k_{a}\right.$ and $\left.k_{m}\right)$. de Vries (1963) provides further information on how to calculate these weighting factors.

The apparent thermal conductivity as a function of soil water content is illustrated in Fig A.2 for a temperature of $25{ }^{\circ} \mathrm{C}$.

Partial differential equations The transport equations (EqA.1) - Eq(A.5) combined with the parameter functions (Eq(A.6) - Eq(A.15) ) lead to a coupled system of two partial differential equations, that need to be solved for the two unknowns - temperature $T$ and matric potential $\psi$ : 


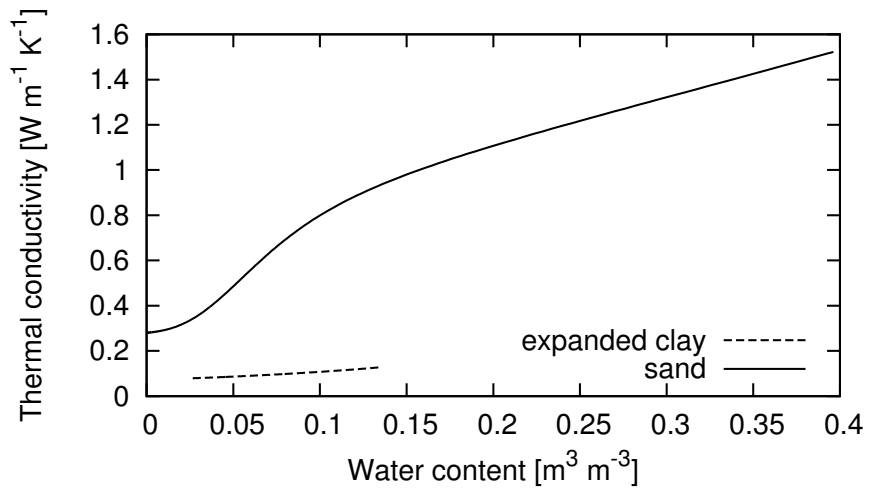

Figure A.2: Thermal conductivity as a function of water content at a temperature of $25{ }^{\circ} \mathrm{C}$ for the parameters of table A.1.

$$
\begin{array}{ll} 
& \rho_{w} \frac{\partial}{\partial t} \theta(\psi) \\
=\quad & \nabla \cdot[K(\psi) \nabla(\psi+g z) \\
+\quad & \left.D_{v} h c_{v}^{\prime} \frac{M_{w}}{R T} \nabla \psi+D_{v} h s \nabla T\right] \\
& \left(C_{m} \rho_{m} x_{m}+C_{w} \rho_{w} \theta(\psi)\right) \frac{\partial T}{\partial t} \\
=\quad & \nabla \cdot[\lambda \nabla T \\
+\quad & \left(T C_{w}\right) K(\psi) \nabla(\psi+g z) \\
+\quad & \left.\left(L+T C_{w}\right) D_{v} h c_{v}^{\prime} \frac{M_{w}}{R T} \nabla \psi+\left(L+T C_{w}\right) D_{v} h s \nabla T\right]
\end{array}
$$

This system of equations is solved under the following boundary conditions.

\section{A.2.2 Atmospheric boundary conditions}

Both weather as well as soil surface conditions determine the flow of energy and water across the interface between soil and atmosphere - the upper boundary in our model.

Water Liquid water flow at the upper surface is given by precipitation $\mathrm{Pr}$. In case of irrigation it has to be added to the precipitation.

Vapor Vapor flow is given by evaporation, with an evaporation rate $E$ of:

$$
E=\frac{c_{v s}-c_{v a}}{r_{v}+r_{s}}
$$

where $E>0$ means that water is evaporating from the soil to the air, in case of dew it is $E<0 . r_{v}$ is aerodynamic resistance for water vapor transfer, $r_{s}$ soil surface resistance 
for water vapor transfer, $c_{v a}$ the atmospheric vapor concentration at height $z_{r e f}$ and $c_{v s}$ soil surface vapor concentration. The surface is at height $z_{0}=0 \mathrm{~m}$.

As described in Griend and Owe (1994) soil surface resistance depends on the water content in the top soil layer:

$$
r_{s}=10 \mathrm{~s} \mathrm{~m}^{-1} \exp \left(0.3563\left(\theta_{\min }-\theta_{\text {top }}\right)\right)
$$

where $\theta_{\text {min }}=15 \%$ is an empirical parameter and $\theta_{\text {top }}[\%]$ is the water content in the top 1 cm layer.

Aerodynamic resistance $r_{v}$ depends on wind speed, level of turbulence, soil surface roughness and thermal stratification of the boundary layer. For the calculation of $r_{v}$ we will follow the description of Campbell (1985):

$$
r_{v}=\frac{1}{u^{*} k}\left[\ln \left(\frac{z_{\text {ref }}-d_{0}+z_{H}}{z_{H}}\right)+\Phi_{H}\right]
$$

where $u^{*}$ is friction velocity, $k$ von Karman's constant, $z_{\text {ref }}$ the height where air temperature is measured, $d_{0}$ is the zero plane displacement for the surface, $z_{H}$ is called surface roughness parameter for heat and $\Phi_{H}$ is a stability correction factor for heat.

Friction velocity is:

$$
u^{*}=u k\left[\ln \left(\frac{z_{\mathrm{ref}}-d_{0}+z_{M}}{z_{M}}\right)+\Phi_{M}\right]^{-1}
$$

where $z_{M}$ is called surface roughness parameter for momentum and $\Phi_{M}$ is a stability correction factor for momentum. The parameters for typical crop surfaces are:

$$
\begin{aligned}
d_{0} & =0.77 h_{c} \\
z_{M} & =0.13 h_{c} \\
z_{H} & =0.2 z_{M}
\end{aligned}
$$

where $h_{c}$ is the height of the surface elements, in our simulations of bare soil this is around: $h_{c}=0.01 \mathrm{~m}$.

The stability correction factor depends on the difference between air and soil temperature: When soil temperature is colder than air, the atmosphere is stable. But when soil is warmer, warmed-up air moves upwards and leads to turbulence. Therefore the stability correction factors depend on a stability parameter $\zeta$ that accounts for this phenomenon:

$$
\zeta=\frac{-k z_{r e f} g H}{C_{h} T_{K} u^{* 3}}
$$


where $H$ is the sensible heat flux in the boundary layer. $H<0$ means that sensible heat flux is negative, hence soil temperature is lower than air temperature and the atmosphere is in stable conditions. The stability correction factor can now be calculated:

$$
\Phi_{H}= \begin{cases}4.7 \zeta & \text { if } H<0 \\ -2 \ln \left[\frac{1}{2}\left(1+[1-16 \zeta]^{\frac{1}{2}}\right)\right] & \text { if } H \geq 0\end{cases}
$$

and

$$
\Phi_{M}= \begin{cases}\Phi_{H} & \text { if } H<0 \\ 0.6 \Phi_{H} & \text { if } H \geq 0\end{cases}
$$

The stability parameter $\zeta$ depends on the sensible heat flow $H$ at the upper boundary, $H$, however, depends in turn on $\zeta$. Therefore $\zeta$ and the corresponding heat flow $H$ have been calculated in 3 for - loops: for the first for - loop $\Phi_{H}=0$ and $\Phi_{M}=0$ have been set and in the following for - loops $\Phi_{H}, \Phi_{M}, \zeta$ and $H$ (as described below) were updated .

Energy: Thermal energy flow at the surface boundary consists of sensible heat, latent heat, a convective part transported by liquid water during precipitation and radiative energy flow:

$$
q_{h}=H+L E-T C_{w} P r+R_{n e t}
$$

where positive $q_{h}$ means that net thermal energy is moving from the soil surface upwards. $H$ is sensible heat flow, $E$ evaporation rate, $L$ latent heat of vaporization, $T$ air temperature, $\operatorname{Pr}$ precipitation rate and $R_{n e t}$ is net radiation from the soil surface upwards, that means radiation from sun and atmosphere contribute negatively and radiation from the soil is positive.

Sensible heat flow is:

$$
H=\frac{C_{h}\left(T_{s}-T_{a}\right)}{r_{h}}
$$

where $r_{h}=r_{v}$ is aerodynamic resistance for heat which is equal to aerodymic resistance for vapor. $T_{a}$ is temperature of the air at reference height $z_{r e f}$ and $T_{s}$ the soil surface temperature.

The radiative energy exchange at the soil surface is composed of the absorbed short-wave radiation from the sun $R_{\text {sun }}$, the long-wave radiation from the atmosphere $R_{\text {atm }}$ and the long-wave radiation emitted by the soil surface $R_{\text {soil }}$.

The energy emitted from the soil is given by Stephan Boltzmann's law:

$$
R_{\text {soil }}=\epsilon_{s} \sigma T_{K, \text { soil }}^{4}
$$

where $\epsilon_{s}$ is the soil emissivity, $\sigma$ Boltzmann's constant and $T_{K, \text { soil }}$ the soil surface temperature in $[\mathrm{K}]$. 
The absorbed short-wave radiation is measured incoming short-wave radiation $R_{\text {meas }}$ weighted by the absorptivity ( 1 - albedo):

$$
R_{\text {sun }}=(1-\text { albedo }) R_{\text {meas }}
$$

For the albedo an average value for bare soil of 0.2 was chosen.

Long-wave radiation from the atmosphere is:

$$
R_{a t m}=\epsilon_{a c} \sigma T_{K, a i r}^{4}
$$

where $T_{K, \text { air }}$ is the temperature of the air and $\epsilon_{a c}$ is the atmospheric emissivity, that depends on vapor concentration of the air $c_{v a}$, and fractional cloud cover $c_{1}$ :

$$
\epsilon_{a c}=\left(1-0.84 c_{1}\right) 0.58 c_{v a^{\frac{1}{7}}}+0.84 c_{1}
$$

The fractional cloud cover is related to the transmissivity $T_{t}$ of the atmosphere: $c_{1}=2.33-$ $3.33 T_{t}$ (valid for $0<c_{1}<1$ ). The transmissivity of the air is a daily value calculated by comparing the measured daily incoming short-wave radiation $R_{\text {meas,day }}$ to the potential incoming short wave radiation $R_{\text {pot,day }}$ :

$$
T_{t}=\frac{R_{\text {meas }, \text { day }}}{R_{\text {pot }, \text { day }}}
$$

The potential incoming short wave radiation during one day depends on latitude $\phi$, solar declination $\delta$ and half daylength $h_{s}$ (in $\mathrm{rad}$ ):

$$
R_{\text {pot }, \text { day }}=117.5 \mathrm{MJ} \mathrm{m}^{-2}\left[h_{s} \sin \phi \sin \delta+\cos \phi \cos \delta \sin h_{s}\right] / \pi
$$

where half daylength is given by $\cos h_{s}=-\tan \phi / \tan \delta$ and the solar declination $\delta$ depends on the day of the year, $d$ :

$$
\sin \delta=0.399 \sin [4.87+0.0172 d+0.0335 \sin (6.22+0.0172 d)]
$$

Finally the total radiative energy flux is:

$$
R_{\text {net }}=R_{\text {soil }}-R_{\text {sun }}-R_{\text {atm }}
$$

\section{A.2.3 Boundary conditions at cable surface}

Since the inside of the cable is hydraulically isolated from the soil, the cable's boundary conditions are no-flow conditions for water and the thermal energy flow is:

$$
q_{\text {cable }}=\frac{J}{2 \pi r_{c}}
$$

where $J\left[\mathrm{~W} \mathrm{~m}^{-1}\right]$ is average electric energy loss of the cable and $r_{c}$ radius of the cable. 


\section{A.3 Simulations}

\section{A.3.1 Applications}

The model was applied to three different cases:

1. to a downscaled experiment, see Minopoli (2007/2008) and de Lieto Vollaro et al. (2014), to test and validate the model

2. to a simplified geometry to demonstrate the key role of hydraulic processes in the evolution of the cable temperature

3. to an underground transmission line system with realistic extensions and under real weather conditions.

In the following these three cases are explained in more details. The shape and dimensions of the simulation domain are depicted in Fig A.3.

Test on a downscaled experiment The experimental data is taken from Minopoli $(2007 / 2008)$ and de Lieto Vollaro et al. (2014). They monitored the temporal evolution and spatial distribution of temperature within a buried electrical cable system. The classical extensions of a transmission line system have been downscaled by a factor of ten to obtain a laboratory suitable size, see Fig A.4. The set-up consists of a box with a trench. Thirtyone thermocouples monitored the temperature and their positions are indicated in Fig A.4.

The cable is a hollow cylinder made of stainless steel with an outer radius $r_{c}=2.5 \times 10^{-3}$ m. An electric current flowing through the cable generates thermal energy. By measuring and controlling current and tension the thermal energy loss of the cable is monitored and fixed to a certain value. The box is filled with expanded clay aggregates (a material often used in constructions because of its low density) and the trench with different materials. We will focus only on the case of a sandy soil as backfill material of the trench (run 1,2,5,6 in Minopoli $(2007 / 2008))$.

The soil properties are typical values (Campbell and Norman, 1998) of a sandy soil and a clay soil with a reduced saturated water content, see Tab A.1 and Fig A.1, the bulk densities have been taken from de Lieto Vollaro et al. (2014).

Note that the measured thermal conductivity values: $\lambda=0.14 \mathrm{~W} \mathrm{~m}^{-1} \mathrm{~K}^{-1}$ (expanded clay aggregates) and $\lambda=0.3 \mathrm{~W} \mathrm{~m}^{-1} \mathrm{~K}^{-1}$ (dry sand) fit very well with our model even though the measured values of thermal conductivity have not been included in the model: in our simulation the values of thermal conductivity are in a range of $0.08 \mathrm{~W} \mathrm{~m}^{-1} \mathrm{~K}^{-1}<\lambda<0.14$ 
a)

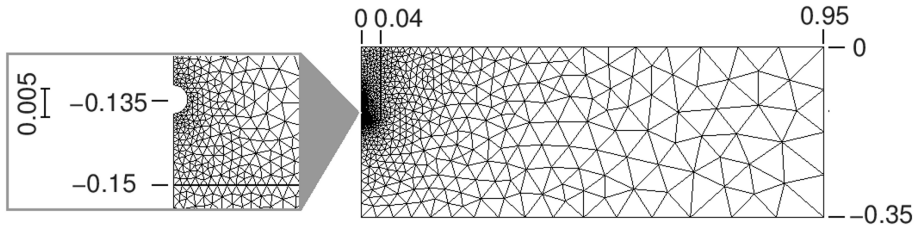

b)

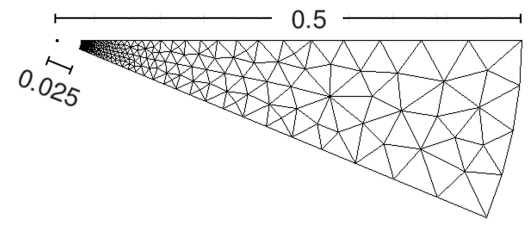

c)

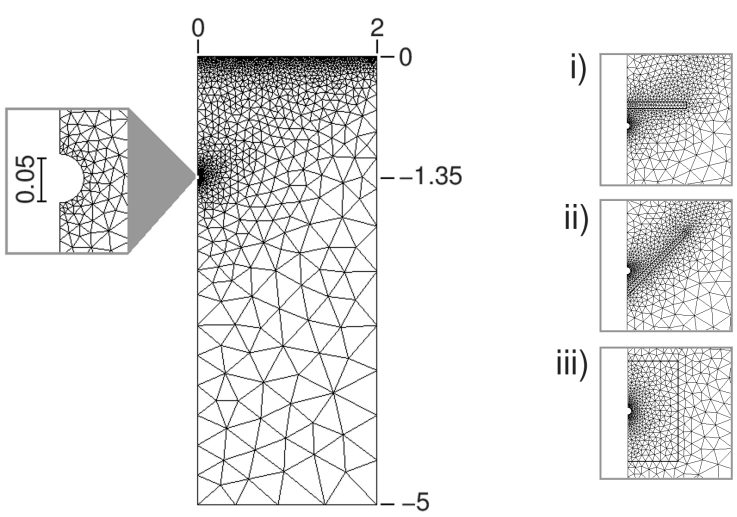

Figure A.3: Shape of the domain and spatial discretization used in the simulations: a) down-scaled experiment b) simplified cylindrical geometry with realistic extensions c) realistic situation with a local grid refinement depending on the scenario: i) concrete slab, ii) hydraulic barrier of v-shape and iii) silt layer.

Table A.1: Hydraulic properties of the soil types used in the simulation of the downscaled experiment. Values for $\psi_{e}, b, K_{s}$ and clay content are from Campbell and Norman (1998), the bulk densities $\rho_{b}$ have been measured by de Lieto Vollaro et al. (2014).

\begin{tabular}{lccccc}
\hline & $\begin{array}{c}\psi_{e} \\
{\left[\mathrm{~J} \mathrm{~kg}^{-1}\right]}\end{array}$ & $b$ & $\begin{array}{c}K_{s} \\
{\left[\mathrm{~kg} \mathrm{~s} \mathrm{~m}^{-2}\right]}\end{array}$ & Clay & $\begin{array}{c}\rho_{b} \\
{\left[\mathrm{~kg} \mathrm{~m}^{-3}\right]}\end{array}$ \\
\hline sand & -0.7 & 1.7 & $5.8 \times 10^{-3}$ & 0.03 & 1,600 \\
expanded clay & -3.7 & 7.6 & $1.7 \times 10^{-5}$ & 0.60 & 540 \\
\hline
\end{tabular}



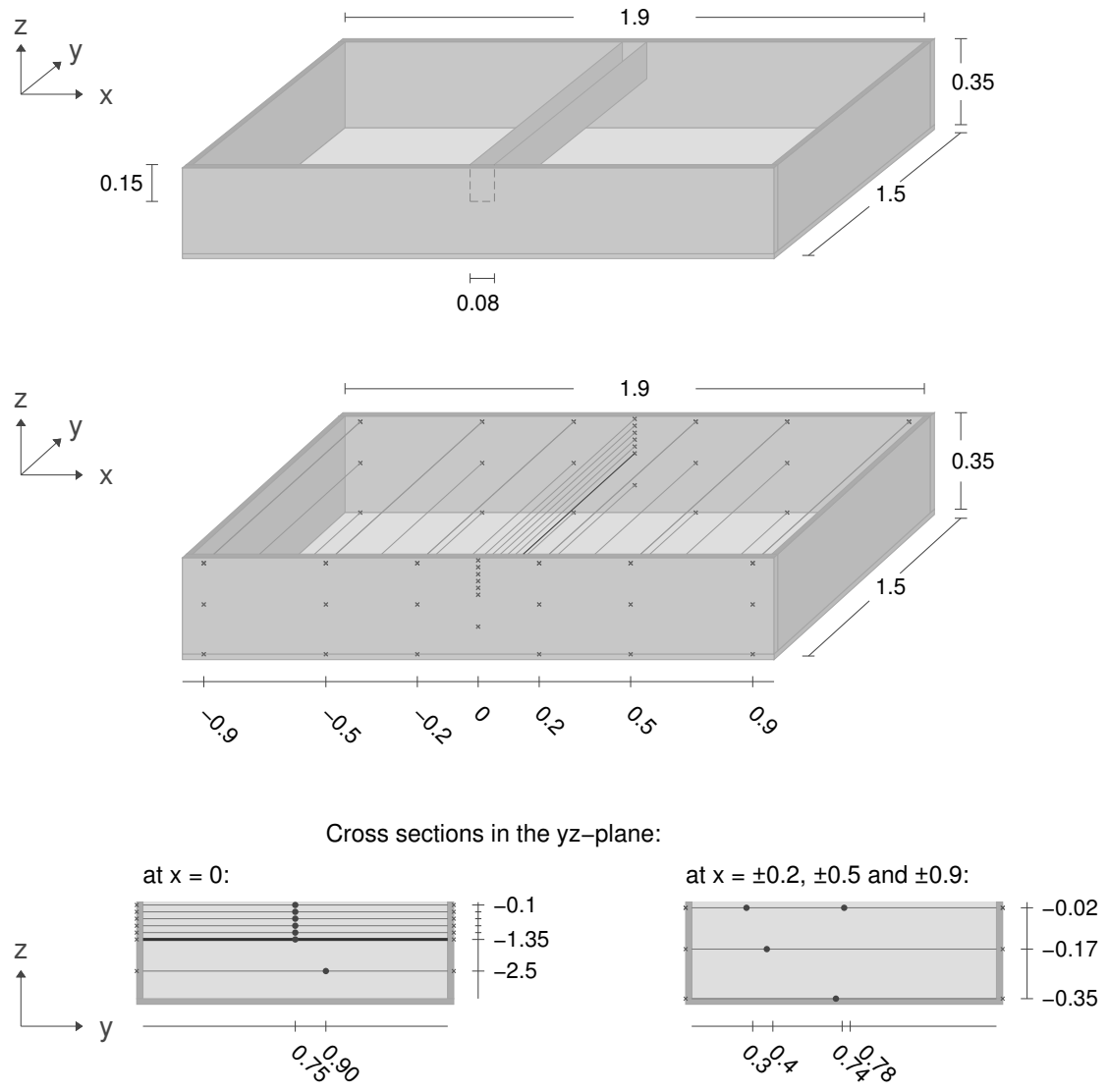

Figure A.4: Top: Dimensions of experimental set-up. Units are [m]. Bottom: The position of the installed thermocouples (filled circles in the cross sections) and the position of the cable (dark line) within the soil-box. Numbers are the corresponding coordinates, where $x=0$ is in the middle of the box, $y=0$ at the front of the box and $z=0$ at the top. Units are $[\mathrm{m}]$. 
$\mathrm{W} \mathrm{m}{ }^{-1} \mathrm{~K}^{-1}$ (clay aggregates) and $0.28 \mathrm{~W} \mathrm{~m}^{-1} \mathrm{~K}^{-1}<\lambda<0.40 \mathrm{~W} \mathrm{~m}^{-1} \mathrm{~K}^{-1}$ (dry sand), see also Fig A.2.

Minopoli (2007/2008) monitored several runs under different room temperature and different thermal energy loss of the cable (see Tab A.3), where each run took around four days.

The initial matric potential was fitted by hand to the experimental data. We obtained a good fit for $\psi=-2,000 \mathrm{~J} \mathrm{~kg}^{-1}$. Since the sandy soil was very dry this value is quite reasonable. The initial temperature was set to the measured room temperature. The room temperatures in the different experimental runs varied between $16^{\circ} \mathrm{C}$ and $24^{\circ} \mathrm{C}$ (see Tab A.3).

Since there was almost no wind in the room we assumed a wind speed of $0.1 \mathrm{~m} \mathrm{~s}^{-1}$. For humidity a value of $h=70 \%$ was used. In the room there is no sunshine, so the radiative energy exchange is given by long-wave radiation emitted from the room and longwave radiation emitted from the soil:

$$
q_{h}=\epsilon_{s} \sigma T_{K, \text { soil }}^{4}-\epsilon_{\text {room }} \sigma T_{K, \text { room }}^{4}
$$

The long wave emissivity of bare soil is usually a value in the range of $0.95-1$. For the simulation we used $\epsilon_{s}=1$ and $\epsilon_{\text {room }}=1$.

The soil box is also emitting radiation at the side and lower boundaries. Therefore we chose EqA.39 as lower and side boundary condition for thermal energy flow. For water and vapor the conditions at the side and lower boundary were no-flow.

The problem was simplified into a 2-dimensional cross section in the xz-plane. For symmetric reasons it is sufficient to solve only one side of the cross section (see Fig A.3), with no-flow condition at the new boundary - the plane of symmetry.

To compare our model to models that do not account for water and vapor flow, we simulated additionally the expected thermal processes under the assumption that heat is only transported by conduction with a thermal resistivity of $0.9 \mathrm{mC} / \mathrm{W}$, a value that is sometimes mentioned for soil thermal conductivity (Campbell and Bristow, 2006; Kellow, 1981). As boundary conditions for heat flow we imposed Eq(A.39), as well.

Simulation based on simplified geometry Aim of these simulations is to demonstrate the impact of water content on the thermal dynamics under simplified conditions. We neglected gravity driven water flow and looked at a radially symmetrical domain with a cable generating energy at the center and with an outside boundary of constant water potential $\psi=\psi_{0}$ and constant temperature $T=7^{\circ} \mathrm{C}$. These values have also been chosen as initial 
condition for temperature and water potential in the entire domain. The radius of the cable (inner boundary of the domain) is a realistic value of $0.025 \mathrm{~m}$. The radius of the outer boundary is $0.5 \mathrm{~m}$. This has been chosen since underground electric cables are often buried in a depths of around $1 \mathrm{~m}$. In this way the entire cylindrical domain would still be completely below surface.

The soil type of the simulation was a sandy soil with properties described in Tab A.1.

The cable generated heat at a constant rate of $J=100 \mathrm{~W} \mathrm{~m}^{-1}$. In nine runs hydraulic and thermal dynamics have been simulated during a 10-days period. The water potential $\psi_{0}$ was a constant value within each run that increased among the runs from $-0.7 \mathrm{~J} \mathrm{~kg}^{-1}$ to -14 $\mathrm{J} \mathrm{kg}^{-1}$.

Since gravity is neglected the entire problem is radially symmetrical and hence it is sufficient to simulate only a sector of the circle with no-flow boundary conditions at the new radial boundaries, see Fig A.3

Simulation of underground cable system under real weather conditions In these simulations diameter, depths and electric loss of the cable correspond to values of real underground electric transmission lines.

As natural soil we imposed the properties of a sandy soil, see Tab A.1. The upper boundary conditions are defined by the weather conditions measured by a weather station (44 $24 \mathrm{~N}, 1128 \mathrm{E}$ ) of the University of Bologna, which is located in the Centonara Watershed, south-east of Bologna, Italy, during the 300 day period from $1^{\text {st }}$ of March 2006 till $26^{\text {th }}$ of December 2006. Precipitation and temperature can be seen in Fig A.5.

At the end of winter the soil is still rather cold and we started the simulation with an initial condition of $7^{\circ} \mathrm{C}$ and for the water potential we set the initial value to $\psi=-6 \mathrm{~J} \mathrm{~kg}^{-1}$ which corresponds for a sandy soil to a water content of around $\theta=0.1$.

Due to symmetry it is sufficient to simulate the half plane on one side of the vertical plane of symmetry through the center of the cable, see Fig A.3. In this way the half circle of the cable together with the plane of symmetry become a new outer boundary. The boundary condition at the plane of symmetry is no-flow.

We set the other side boundary at a distance of $2 \mathrm{~m}$ and the boundary condition to noflow. We tested that the thermal dynamics close to the cable are not sensitive to an increase of the distance towards that boundary. In this way we think choosing no-flow conditions is justified.

If the groundwater table is deeper than a few meters depths the hydraulic conditions are 

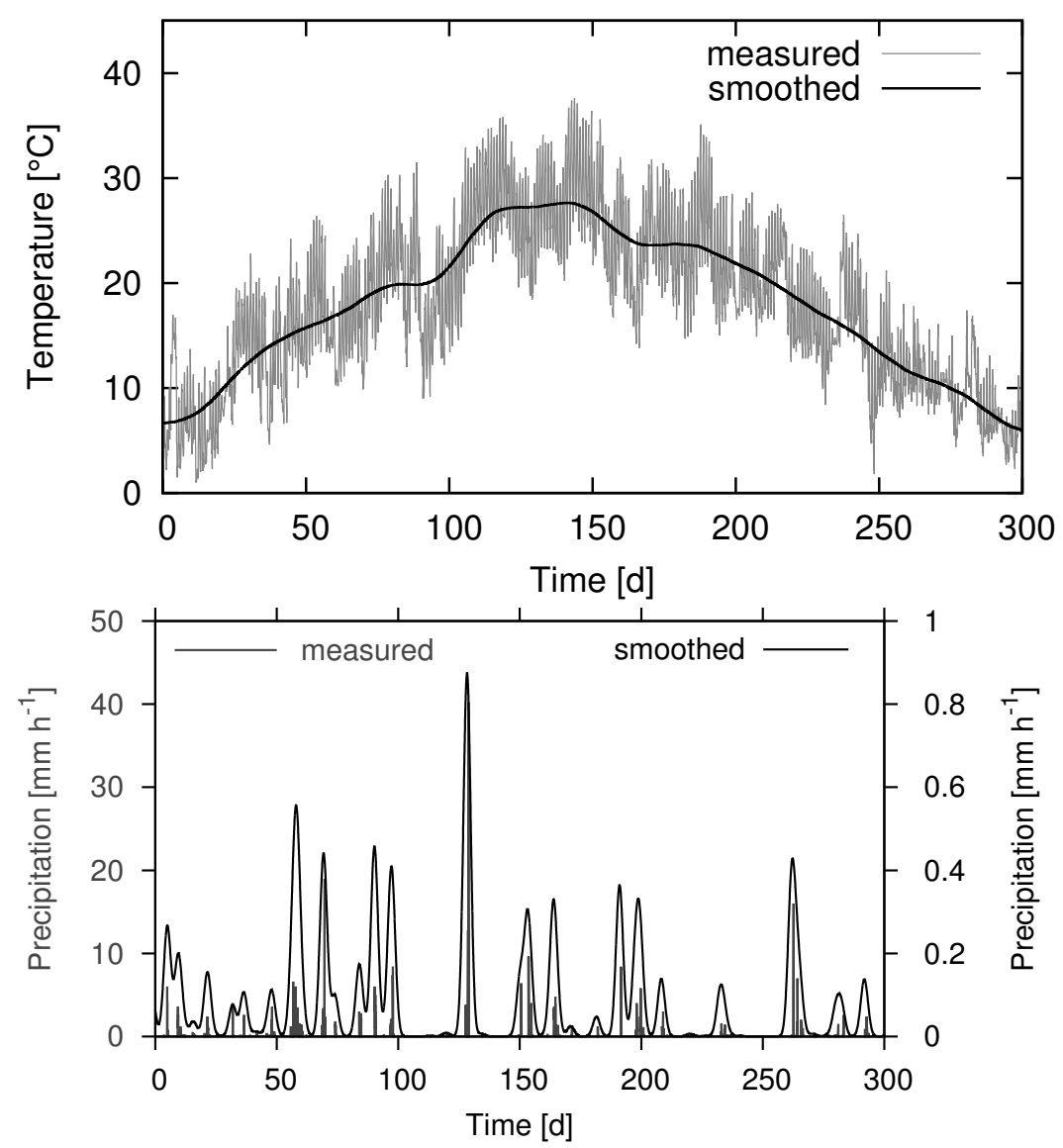

Figure A.5: Weather conditions at the upper boundary. Measured temperature and precipitation and smoothed function of both are shown. They have been smoothed by a convolution with a Gaussian function. The standard deviations of the used Gaussian functions were $6 \mathrm{~d}$ for the temperature plot and $35 \mathrm{~h}$ for the precipitation plot. 
Table A.2: Hydraulic parameters of the different materials used in Run B-D of the weather study.

\begin{tabular}{lccccc}
\hline & $\begin{array}{c}\psi_{e} \\
{\left[\mathrm{~J} \mathrm{~kg}^{-1}\right]}\end{array}$ & $b$ & $\begin{array}{c}K_{s} \\
{\left[\mathrm{~kg} \mathrm{~s} \mathrm{~m}^{-2}\right]}\end{array}$ & Clay & $\begin{array}{c}\rho_{b} \\
{\left[\mathrm{~kg} \mathrm{~m}^{-3}\right]}\end{array}$ \\
\hline $\begin{array}{l}\text { concrete layer (i) } \\
\text { non-permeable }\end{array}$ & -3.7 & 7.6 & $1.7 \times 10^{-7}$ & 0.60 & 2,040 \\
barrier (ii) & -0.7 & 1.7 & $5.8 \times 10^{-7}$ & 0.03 & 1,600 \\
silt loam (iii) & -2.1 & 4.7 & $1.9 \times 10^{-4}$ & 0.15 & 1,600 \\
\hline
\end{tabular}

mainly influenced by weather conditions and not by capillary rise. In this case the condition for matric potential at the lower boundary is free drainage (unit gradient), furthermore we set both vapor as well as thermal energy flow to zero.

The conditions at the remaining boundaries - the cable and the surface - were imposed as described in the theory of section A.2.

In different runs we tested the influence of different structures in the set-up of underground transmission line systems:

A Homogeneous system of a sandy soil.

B Sandy soil as natural soil and a concrete slab above the cable that is recommended (Public Survice Commission of Wisconsin, 2011) to protect the cable against mechanical damage from the surface

C Sandy soil with a hydraulic barrier of v-shape below the cable. This could be realist by an impermeable foil within the soil.

D Sandy soil with a silty soil as backfill material in a rectangular region around the cable.

The hydraulic parameters used for these additional materials are provided in Tab A.2

For all runs heat was generated at the cable with a constant rate of $J=60 \mathrm{~W} \mathrm{~m}^{-1}$.

In two additional runs we investigated the effect of differences in heat dissipation during day and night. In the future these differences might be enhanced by new technologies, like charging electric vehicles (Marshall et al., 2013): Run A' and run D' have the same set-up as run A and run D respectively. The loss of energy from the cable, however, is a sinus curve with a period length of one day, an amplitude of $0.5 \mathrm{~J}$ and an average heat loss of $J=60 \mathrm{~W}$ $\mathrm{m}^{-1}$. 


\section{A.3.2 Numerical Implementation}

For the discretization of $\mathrm{Eq}$ A.16 and $\mathrm{Eq}$ A.17) we applied continuous piecewise linear finite elements coupled with a time stepping scheme. For the spatial discretization we used unstructured grids (see Fig A.3 which have been generated with Gmsh, a mesh generator developed by Geuzaine and Remacle (2009). To improve the accuracy we considered locally refined meshes. After local grid refinement the cell size is very small close to the cable ( a) $0.7 \mathrm{~mm}$; b) $0.8 \mathrm{~mm}$; c) $6 \mathrm{~mm}$ ) and larger in very distant parts (a) $10 \mathrm{~cm}$; b) $6 \mathrm{~cm}$; c) $60 \mathrm{~cm}$ ). In the real weather study (c) there occur in particular close to the surface steep gradients caused by evaporation, the fast changing radiation and temperature conditions and infiltration fronts. Therefore in case (c) the grid has been locally refined also at the surface to an average cell size of $1.8 \mathrm{~cm}$. In order to include the different structures $[B],[C]$ and $[D]$, the mesh has been refined according to the shape and size of the respective structure, see Fig A.3.

For time discretization we used an implicit Euler scheme and an automatically controlled time step with an upper limit of 0.5 hours. For the simulation of the experiment we compared solutions based on an upper limit of $1 \mathrm{~h}$ for the maximum time step to solutions based on an upper limit of $5 \mathrm{~min}$ and we found that the temporal evolution of the cable temperature defers less than $0.01{ }^{\circ} \mathrm{C}$. When the weather conditions at the upper boundary in the realweather simulation change very rapidly the time step was in the size of a few minutes or several seconds.

In each time step the non-linear equations were solved with a Newton method. The Newton equations have been solved with a bi conjugate gradient stabilized solver (BiCGSTAB) and SSOR preconditioning. The implementation is based on PDELab, a discretization module for partial differential equations. PDELab depends on the Distributed and Unified Numerics Environment (DUNE). Further informations about PDELab can be found in PDELab (2014) and Bastian et al. (2010), informations about DUNE are provided in Blatt and Bastian (2007b); Bastian et al. (2008b a) and on the project homepage DUNE (2014).

\section{A.4 Results and Discussion}

\section{A.4.1 Down-scaled Experiment}

The temporal evolution of the cable temperature is shown for all four runs in Fig A.6 and can be compared to the experimental data. The temperatures increased very fast during the first hours and after $10 \mathrm{~h}$ they have almost reached their final equilibrium value. Coupled 


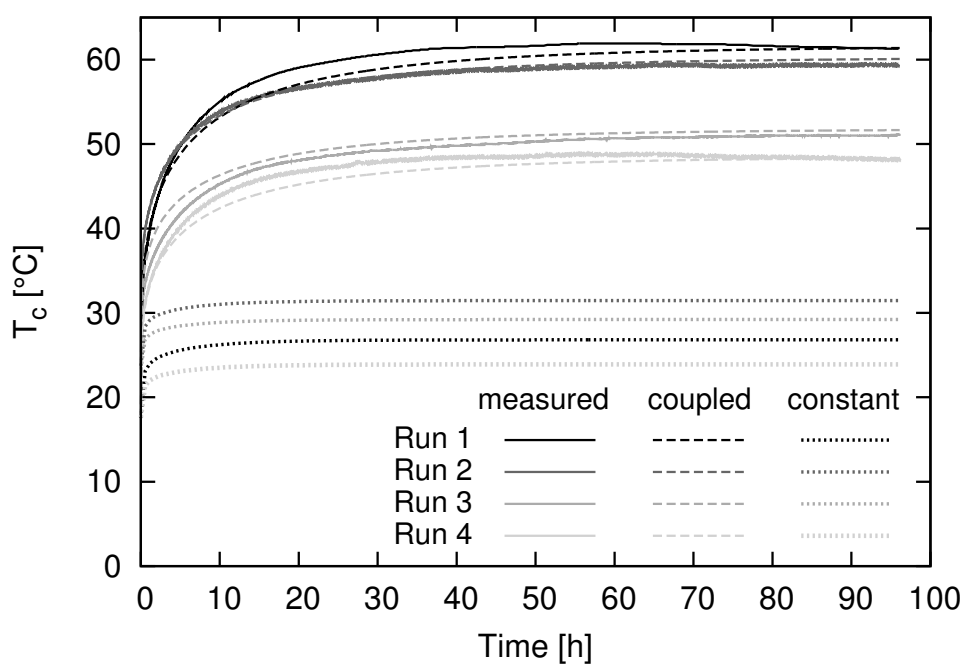

Figure A.6: Temporal evolution of the cable's temperature during the 4-day measurement period for 4 different runs. For each of them measured data (continuous line) and simulated based on the coupled program (dashed line) and based on the assumptions of a constant thermal resistivity of $0.9 \mathrm{mC} / \mathrm{W}$ (dotted line) are plotted.

model and measurements lead to similar results, while the assumption of a constant thermal resistivity of $0.9 \mathrm{mC} / \mathrm{W}$ can lead to an error of more than $30^{\circ} \mathrm{C}$ in the calculated temperature of the cable.

The corresponding values of the cable's temperature after the 4 days can be found in Tab A.3.

A comparison of the spatial distribution of temperature for run 1 is provided in Fig A.7.

Also in the spatial distribution coupled model and measurements were in good agreement while the assumption of a resistivity of $0.9 \mathrm{~m}^{\circ} \mathrm{C} / \mathrm{W}$ severely underestimated the temperature in the soil.

\section{A.4.2 Simplified geometry}

For the simulated 10 day-period the evolution of temperature and matric potential at the cable can be found in Fig A.8. Cable's temperature increased for all initial conditions most strongly during the first day and for most of them it has reached after the second day almost its final value. The initial conditions $\psi=-0.7 \mathrm{~J} \mathrm{~kg}^{-1}$ to $\psi=-12 \mathrm{~J} \mathrm{~kg}^{-1}$ led to an almost constant cable temperature from day 6 till day 10 (change less than $0.5^{\circ} \mathrm{C}$ in five days). However, the initial condition of $\psi=-14 \mathrm{~J} \mathrm{~kg}^{-1}$ led to a change of more than $1^{\circ} \mathrm{C}$ from day 6 till the end of day 10 . 
Table A.3: Generation of cable energy at the cable, ambient air temperature and temperature at the cable after 4 days obtained by: experiment, simulation based on coupling of liquid water, vapor and heat and simulation based on the recommended value for thermal resistivity of $0.9 \mathrm{mC} / \mathrm{W}$.

\begin{tabular}{cccccc}
\hline & $J\left[\mathrm{~W} \mathrm{~m}^{-1}\right]$ & $T_{a}\left[{ }^{\circ} \mathrm{C}\right]$ & \multicolumn{3}{c}{$T_{c}\left[{ }^{\circ} \mathrm{C}\right]$} \\
& & & meas. & coupl. & const. \\
\hline Run 1 & 12.3 & 17.6 & 61.5 & 61.4 & 26.8 \\
Run 2 & 10.3 & 23.8 & 59.4 & 60.1 & 31.5 \\
Run 3 & 7.9 & 23.4 & 51.1 & 51.7 & 29.2 \\
Run 4 & 8.3 & 17.7 & 48.2 & 48.4 & 23.9 \\
\hline
\end{tabular}

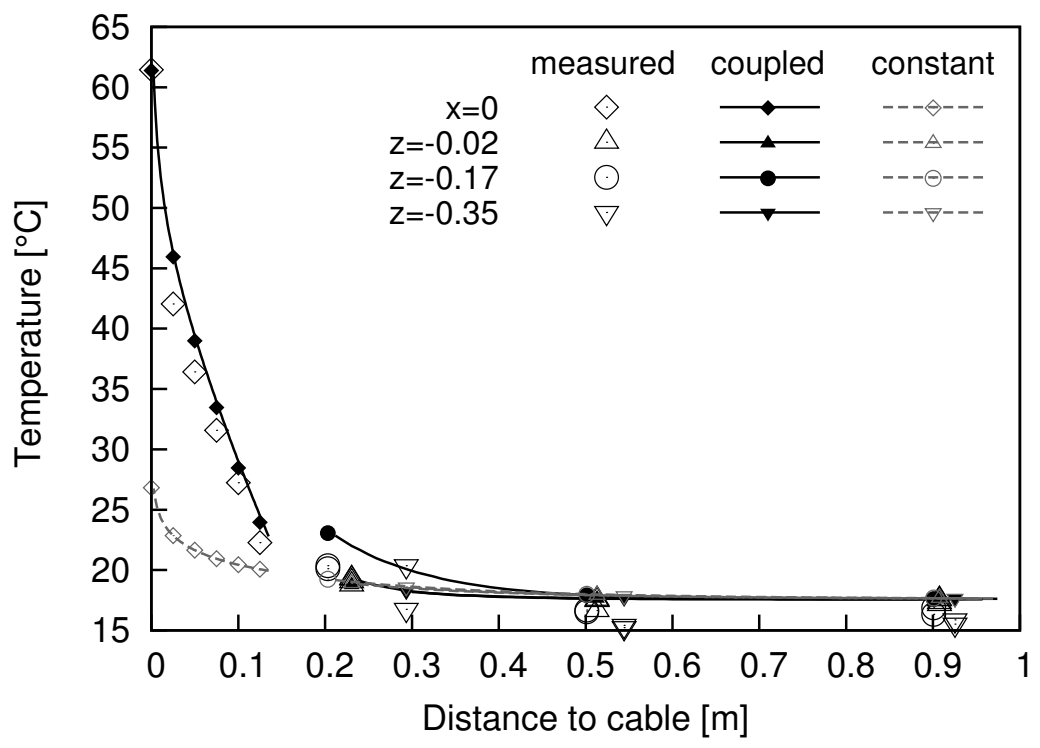

Figure A.7: Spatial distribution of temperature at the end of run 1. Empty symbols correspond to the measured temperature, black symbols with a continuous line to the simulated data based on the coupled model and empty symbols with dashed line to the simulated data based on the assumption of a constant thermal resistivity of $0.9 \mathrm{mC} / \mathrm{W}$. 

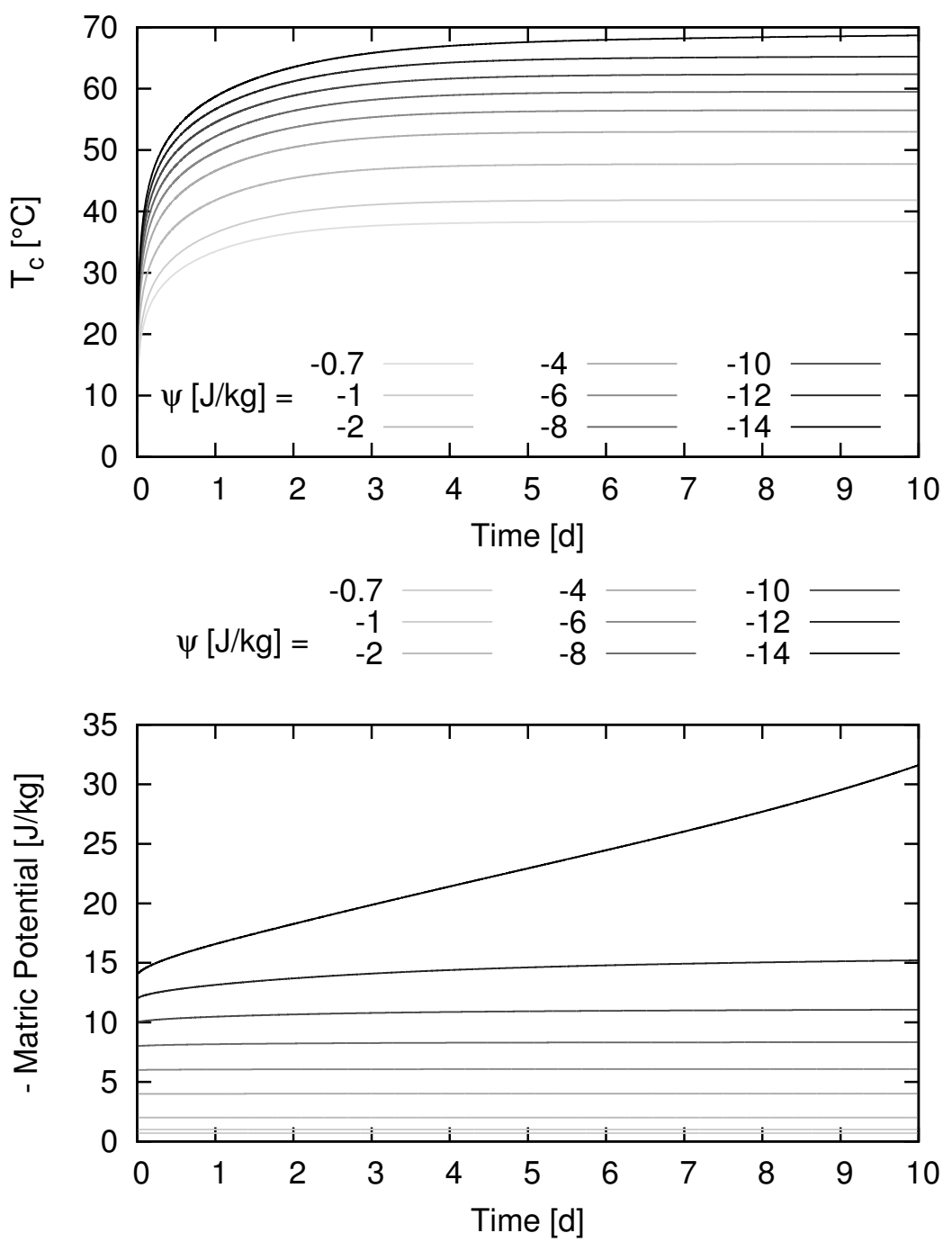

Figure A.8: Evolution of temperature (top) and matric potential at the cable bottom) during the first 10 days under different initial conditions of water potential ranging from $\psi=-0.7$ $\mathrm{J} \mathrm{kg}^{-1}$ to $\psi=-14 \mathrm{~J} \mathrm{~kg}^{-1}$. 
When looking at the matric potential one can observe a similar behaviour: for an initial condition of $\psi=-14 \mathrm{~J} \mathrm{~kg}^{-1}$ the matric potential kept decreasing also at day 10. For the other initial conditions, however, it stayed more or less constant after day 2.

An explanation for this behaviour is that the thermal energy emitted from the cable was heating up the soil around the cable. Due to the increased temperature liquid water evaporated. The increased water vapor concentration led to a vapor flow from the cable towards the distant regions. The vapor flow led to a reduction of liquid water content close to the cable and reduced the matric potential there. The resulting gradient in matric potential caused a liquid water flow towards the center. According to Eq A.7 and Fig A.1 the hydraulic conductivity depends strongly on the matric potential. In our simulations the hydraulic conductivity for an initial matric potential lower than $\psi=-12 \mathrm{~J} \mathrm{~kg}^{-1}$ was still high enough to compensate the vapor flow by liquid water flow. For a matric potential of $\psi=-14 \mathrm{~J} \mathrm{~kg}^{-1}$, however, the conductivity was not high enough, the soil dried out and the matric potential kept decreasing over time.

This explanation is supported by the distribution of temperature and water content at the end of the 10 day period, see Fig A.9. For most initial conditions the distribution of water content after 10 days was more or less constant. However, for $\psi=-14 \mathrm{~J} \mathrm{~kg}^{-1}$ the soil close to the cable was considerably dryer than in the more distant regions.

The temperature distribution shows that depending on the initial conditions of the matric potential the cable temperature varies between $38^{\circ} \mathrm{C}$ and $68^{\circ} \mathrm{C}$.

We see that dry soil leads in several ways to an increase of the temperature at the cable:

1. Dry soil has a lower thermal conductivity (see Eq(A.15)

2. Dry soil has a lower heat capacity (see EqA.5) ).

3. Under steady state conditions the vapor flow from the cable and the liquid water flow towards the cable equilibrate. Therefore the cooling effect of evaporating water close to the cable is limited by the water flow towards the cable that is very low in dry soils because of their low hydraulic conductivity.

Fig A.10 demonstrates the cable's temperature after 10 days as a relation of the initial water content. It is well visible that especially in the dry part the cable's temperature strongly depends on the water content: A difference in water content of $\Delta \theta=0.044 \mathrm{~m}^{3} \mathrm{~m}^{-3}$ can lead to a difference in the cable's temperature of $\Delta T=12.2^{\circ} \mathrm{C}$, therefore stressing the importance of accounting for liquid and vapor flow calculations for cable heat dissipation. 


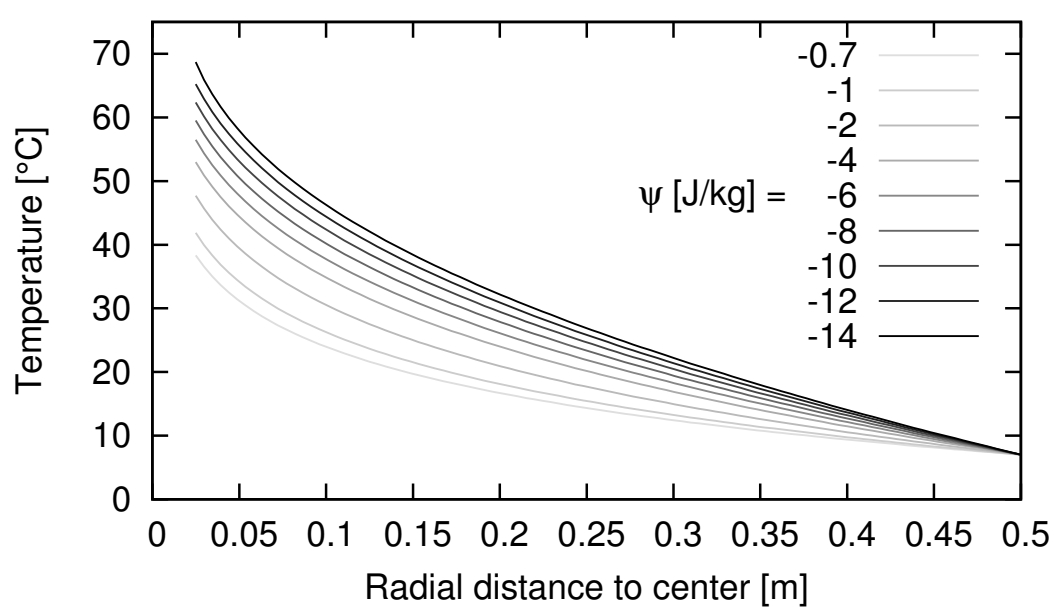

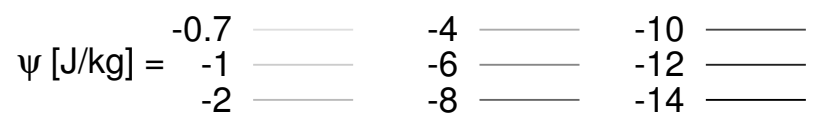

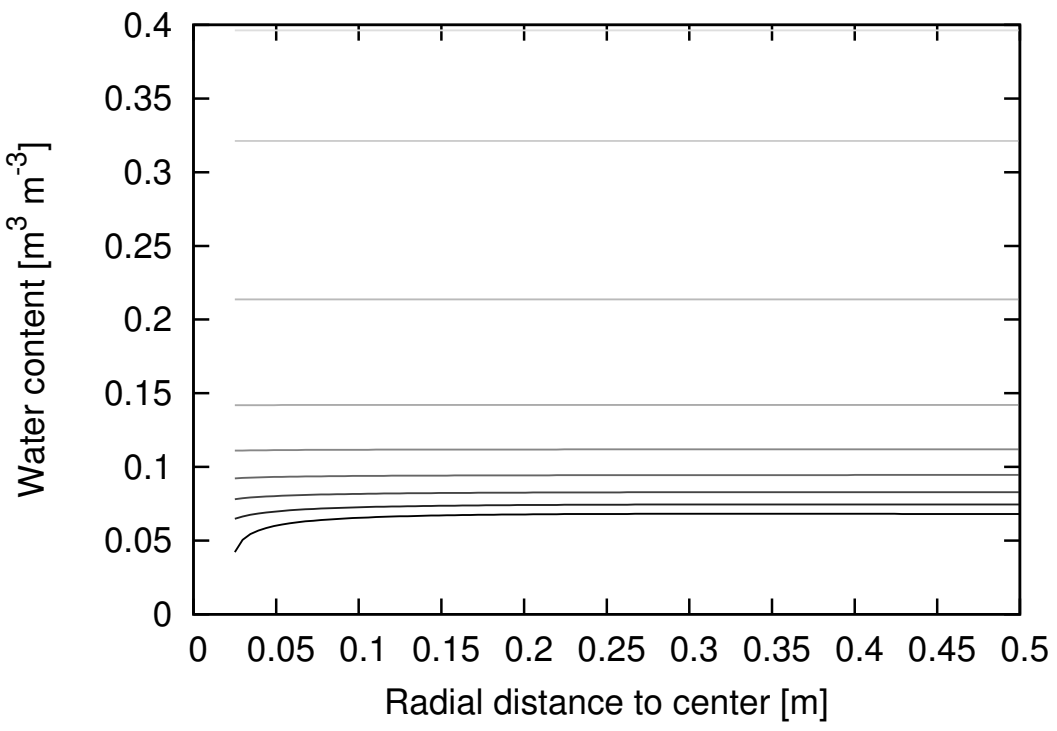

Figure A.9: Radial temperature (top) and radial water content (bottom) distribution after 10 days for different initial water potentials. 


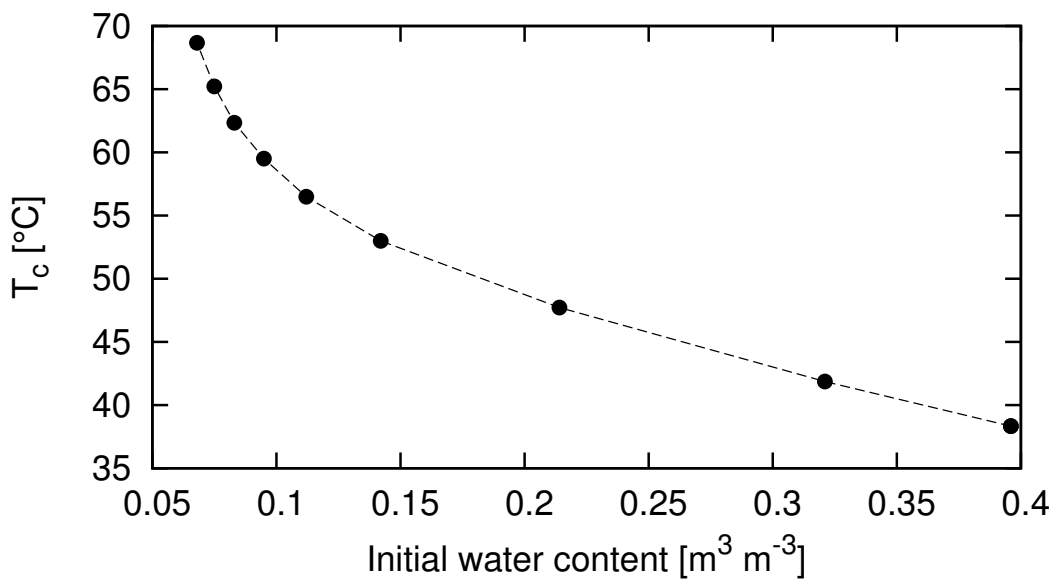

Figure A.10: Simplified geometry simulation: Cable's temperature after 10 days related to initial water content

Table A.4: Average contribution of each component to the energy exchange at the upper surface. Units are $\left[\mathrm{Wm}^{-2}\right]$

\begin{tabular}{lc}
\hline global radiation & -169.6 \\
reflected radiation & 33.9 \\
long-wave downward & -360.0 \\
long-wave upward & 435.1 \\
sensible heat & 48.7 \\
latent heat & 75.0 \\
\hline
\end{tabular}

\section{A.4.3 Simulations under realistic atmospheric conditions}

Fig A.11 provides the contribution of each component to the overall energy exchange between soil and atmosphere during the 300 day period in 2006. Average values for each component can be seen in table A.4.

The evolution of the temperature at the cable is illustrated in Fig A.12. For comparing the results of the different runs with each other, statistical values are provided in Tab A.5. Run $\mathrm{A}$ and run $\mathrm{B}$ have almost the same average temperature, while it is around $2^{\circ} \mathrm{C}$ smaller in run $\mathrm{C}$, in run $\mathrm{D}$ it is even more reduced: around $4{ }^{\circ} \mathrm{C}$ compared to run $\mathrm{A}$. The added daily oscillations in the cable's loss of energy (run A' and run D') do not change the average temperature very much. The variation, however, is increased by a factor of 100. Among the runs with constant loss of energy, run $\mathrm{A}$ has highest variation, run $\mathrm{B}, \mathrm{C}$ and $\mathrm{D}$ have all a similar variation. 

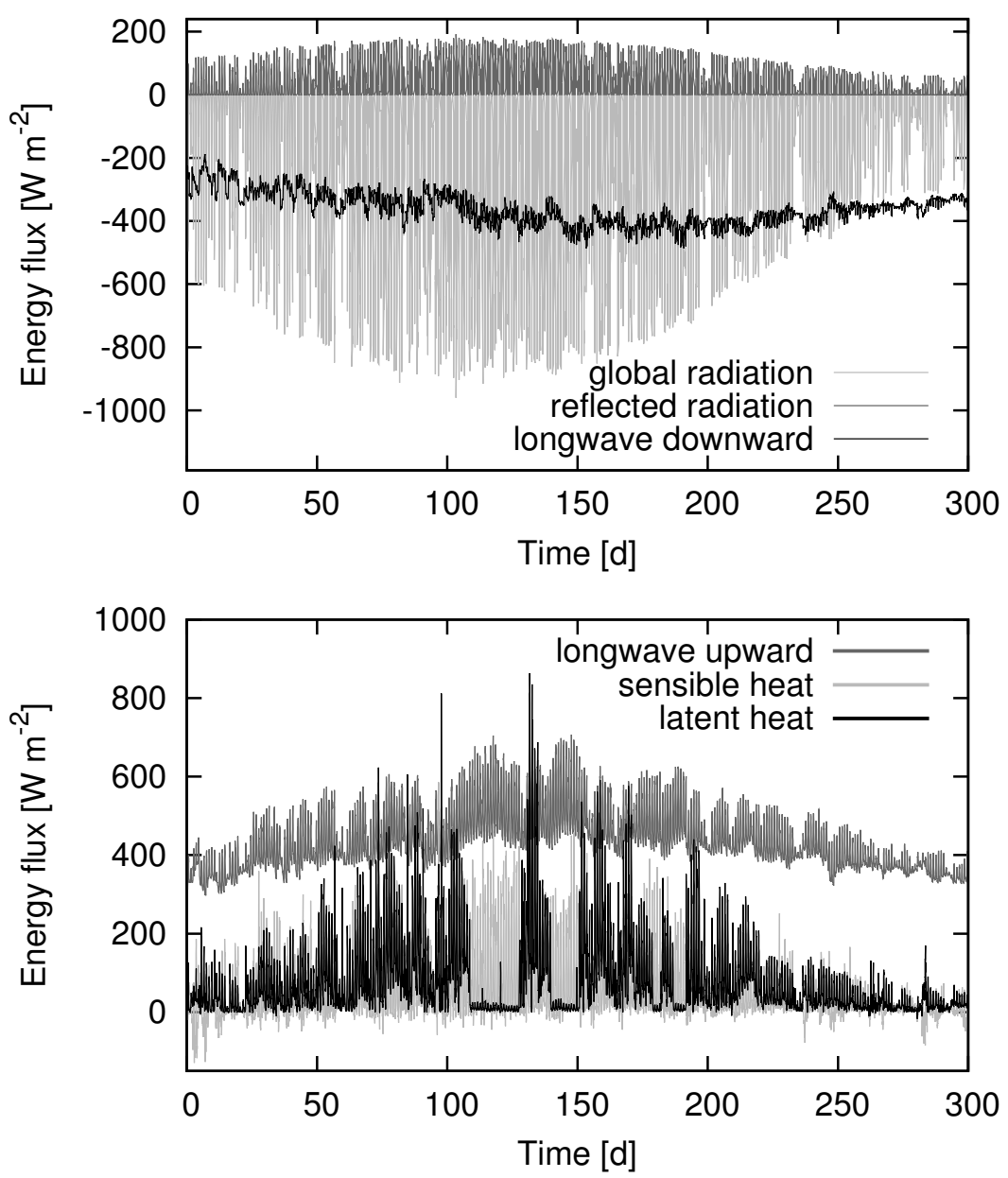

Figure A.11: Thermal energy exchange at the soil surface separated into: sensible heat, latent heat, global solar radiation (measured), radiation reflected by the soil surface, long wave radiation from the atmosphere and long wave radiation emitted from the soil surface

Table A.5: Average temperature $T_{\mathrm{av}}$, variation as an indicator for the oscillation of the cable temperature and the average difference between daily maximum and minimum cable temperature.

\begin{tabular}{cccc}
\hline & $\begin{array}{c}T_{\mathrm{av}} \\
{\left[{ }^{\circ} \mathrm{C}\right]}\end{array}$ & $\begin{array}{c}\operatorname{var}(T) \\
{\left[{ }^{\circ} \mathrm{C}\right]}\end{array}$ & $\begin{array}{c}(\Delta T)_{\mathrm{av}} \\
{\left[{ }^{\circ} \mathrm{C}\right]}\end{array}$ \\
\hline Run A & 59.9 & 65.0 & 0.26 \\
Run B & 59.8 & 58.4 & 0.24 \\
Run C & 57.7 & 60.1 & 0.25 \\
Run D & 55.7 & 57.6 & 0.22 \\
Run A' & 59.8 & $6,760.8$ & 11.6 \\
Run D' & 55.7 & $5,818.8$ & 10.0 \\
\hline
\end{tabular}



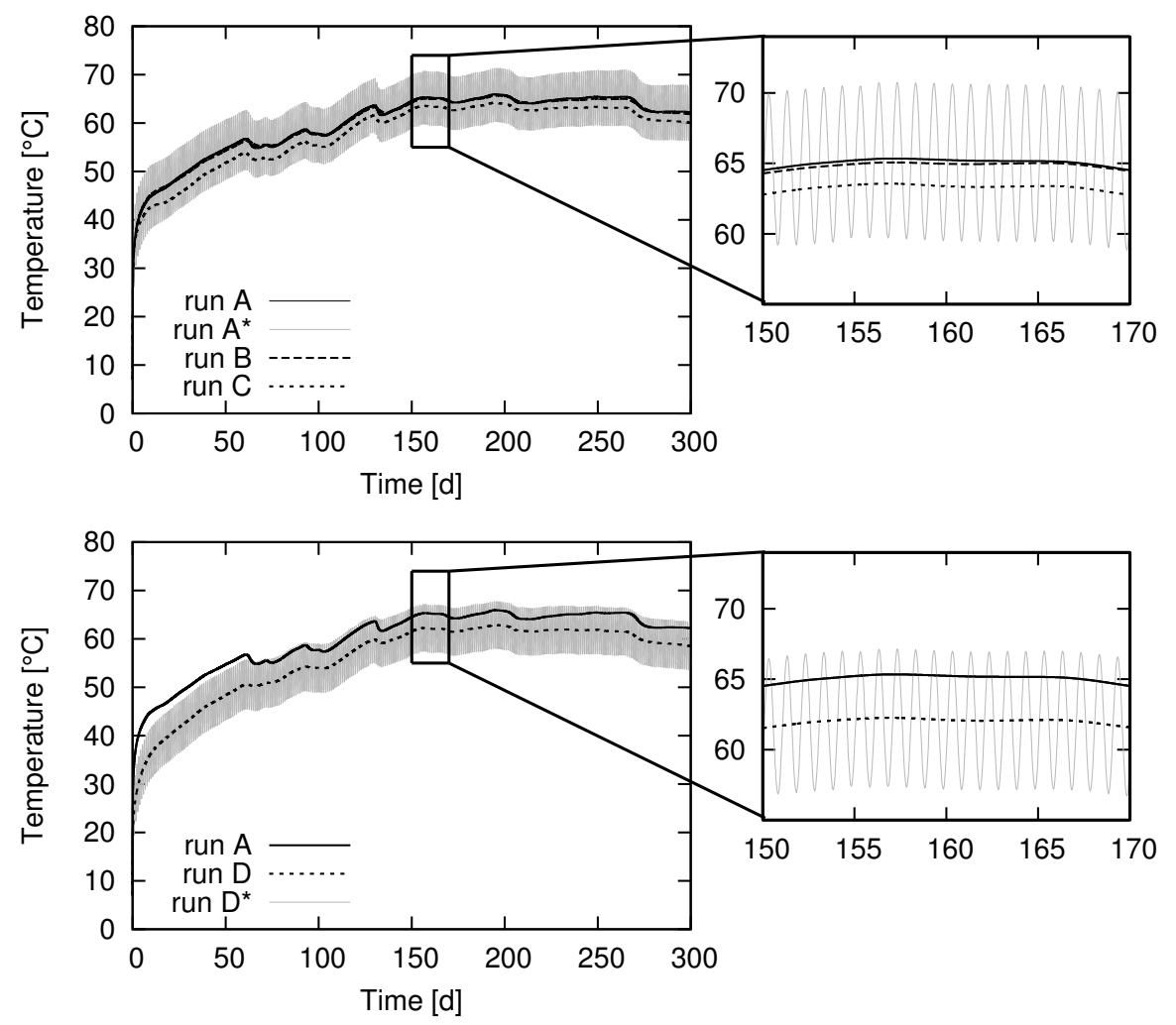

Figure A.12: Temperature at cable surface versus time. (The temperatures of run A and run $\mathrm{B}$ are so close that they can only be distinguished after zooming in.) 

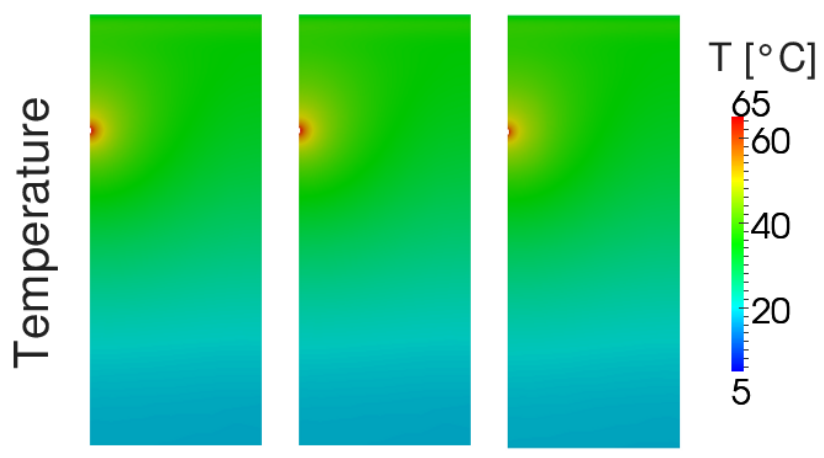

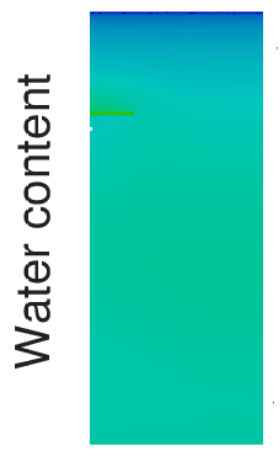

(i)

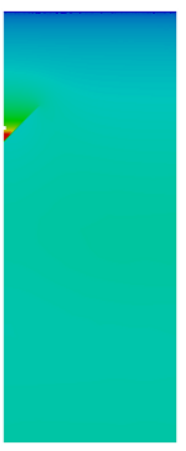

(ii)

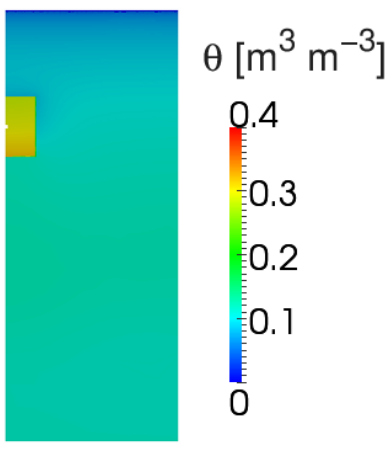

(iii)

Figure A.13: Distribution of temperature and water content at day 150 for the simulated cases (i), (ii) and (iii).

Temperature and water content distribution at day 150 are shown in Fig A.13. Distribution of temperature is quite similar for all the cases (i), (ii) and (iii). In the distribution of water content, however, the added structures are well visible: while the concrete slab (i) doesn't modify the water content around the cable, the impermeable layer (ii) increases the water content around the cable and also the silt layer (iii) increases the water content.

Evolution of the water content at the cable is shown in Fig A.14. Run C and run D have a higher average water content than run $\mathrm{A}$ and run $\mathrm{B}$. While the water content in run A, run $\mathrm{B}$ and run $\mathrm{D}$ is rather stable, it changes more in run $\mathrm{C}$.

That means for this study:

1. The slab of concrete used in underground cable construction against mechanical damage does not negatively influence the average temperature and reduces the variations of the cables temperature.

2. Adding an impermeable layer below the cable keeps the water content around the cable higher and therefore also the thermal conductivity and heat capacity. In this way it leads to a lower average temperature of the cable and less variations. 


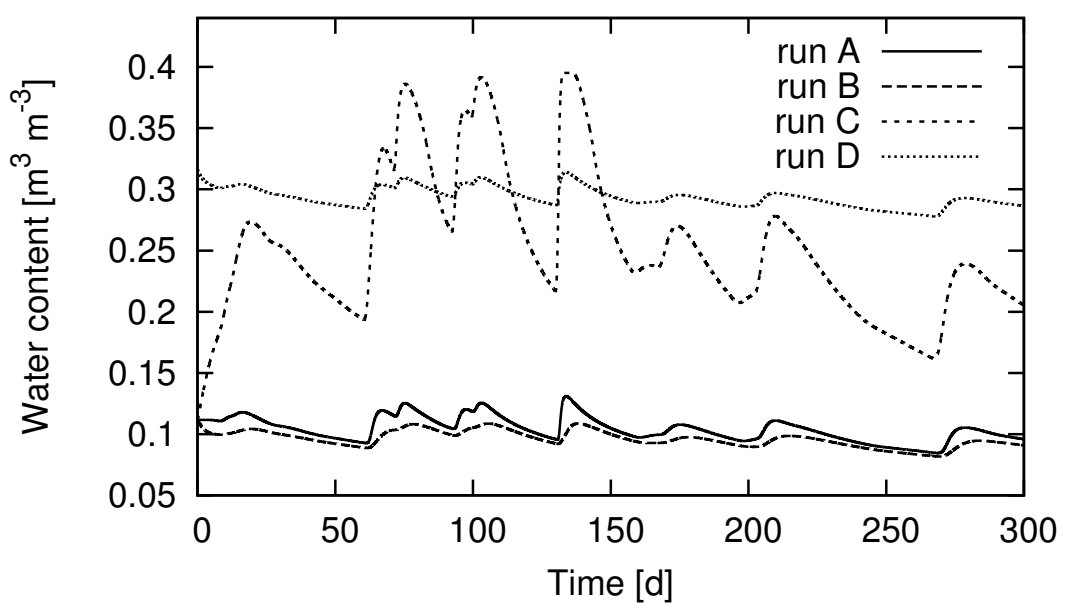

Figure A.14: Water content at cable surface related to time

3. Also a silt layer around the cable can reduce both: average cable temperature as well as variation. This is because of the higher water holding capacity of this soil type, that is finer compared to a sandy soil.

Fig A.15 demonstrates the strong dependence of thermal dynamics at the cable on weather conditions: The air temperature defines - with a delay of 50 days - the seasonal evolution of cable temperature, while the precipitation is strongly correlated - with a delay of 3.15 days - to the momentary changes of the temperature.

\section{A.5 Discussion}

In this study a numerical model of heat dissipation of underground transmission line system including coupled heat, water and vapor flow was presented. In the first part the model was validated by simulating a down-scaled experiment. It was furthermore demonstrated that a model based on a constant value of thermal resistivity of $0.9 \mathrm{~m}^{\circ} \mathrm{C} / \mathrm{W}$ can severely underestimate the cable temperature by more than $30^{\circ} \mathrm{C}$.

Secondly simulations with a simplified geometry demonstrate that good knowledge about the hydraulic dynamics in the soil are essential for an appropriate estimation of the evolution of the cable temperature.

The life-time of an electrical underground transmission line system is very sensitive to both average temperature and fast changes of temperature. Therefore wrong estimates of the cables temperature can lead to severe errors in the estimated life-time of more than 10 years (usually estimates of the total life-time are around 70-80 years (Jones and McManus, 2010)). 

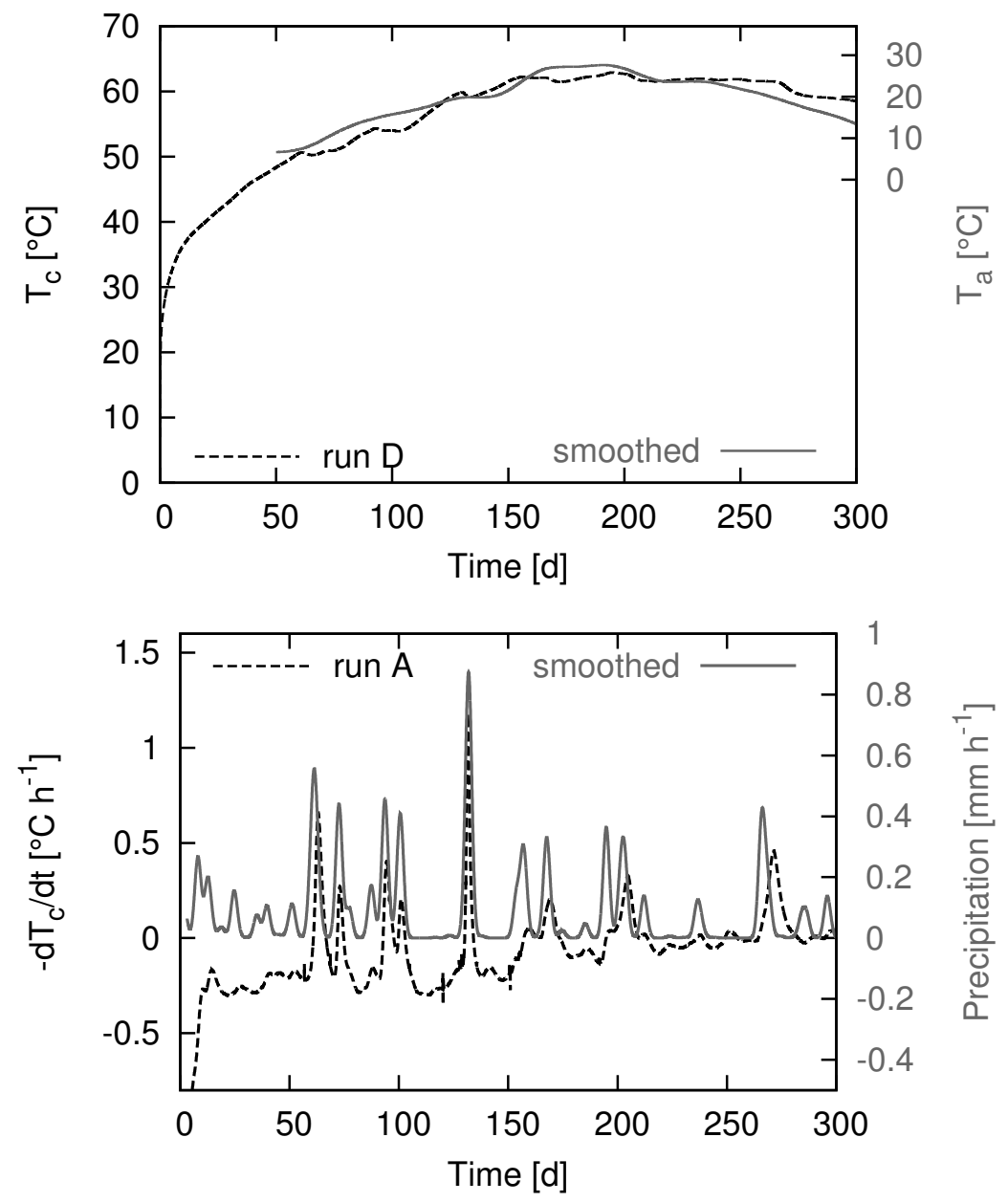

Figure A.15: Top: Cable's temperature for run D and smoothed measured air temperature. The smoothed line is also shown in figure A.5 but here it is shifted forward by 50 days. Bottom: Negative change of the cable's temperature $-d T / d t$ for run A and smoothed precipitation. The smoothed curve is the same as the one shown in Fig A.5 but it is now shifted forward by 3.15 days. 
Finally simulations based on real weather data revealed on one hand that seasonal air temperature defines the seasonal evolution of cable temperature, while precipitation is strongly related to changes in cable temperature on a time scale of hours to a few days. Simulations under consideration of additional structures (concrete slab, impermeable v-shape layer, silt layer) showed that already these simple test cases can reduce average temperature and variation of temperature by some degrees and in this way increase the system's life time compared with the homogeneous example.

This study demonstrated that during construction of an electric underground transmission line system it is fundamental to consider coupling of liquid water, vapor and heat flux when estimating the temperature and expected life-time of the system.

While we demonstrated here that the thermal processes are strongly influenced by weather conditions it might be interesting to compare the differences among various weather conditions at different sites and in different years.

In general, a correct simulation, allows for a better design of cable installations. In particular it allows for choosing the correct cable specifics, the installation depth and the surrounding backfill. These choices may change depending on the properties of the natural soil and the weather conditions.

A limitation of this study is that when soil becomes very dry and hot, the matric potential decreases strongly. The resulting steep gradients can lead to problems for the numerical solver.

The coupled model has been validated by Bittelli et al. (2008) for a one dimensional evaporation scenario from bare soil and in this study we validated it for the down-scaled experiment of Minopoli (2007/2008); de Lieto Vollaro et al. (2014). A comparison, however, to a realistic underground transmission line system will certainly be a good option for supporting this model.

Overall we think that this model can lead to new, better and less expansive ways to estimate the evolution of the cable temperature of an underground cable system and in this way make a positive contribution in the optimization of the life-time of underground transmission line systems. 


\section{A.6 Nomenclature}

\begin{tabular}{|c|c|c|}
\hline Symbol & Unit & Description \\
\hline$b$ & & shape parameter \\
\hline$c_{1}$ & & fractional cloud cover \\
\hline$c_{v}, c_{v}^{\prime}, c_{v a}, c_{v s}$ & $\mathrm{~kg} \mathrm{~m}^{-3}$ & vapor concentration, saturated, atmospheric, at soil surface \\
\hline$C_{h}$ & $\mathrm{~J} \mathrm{~m}^{-3} \mathrm{~K}^{-1}$ & volumetric heat of air $\left(1.2 \times 10^{3}\right)$ \\
\hline$C_{m}, C_{w}$ & $\mathrm{~J} \mathrm{~kg}^{-1} \mathrm{~K}^{-1}$ & specific heat of minerals $\left(0.9 \times 10^{3}\right)$, water $\left(4.18 \times 10^{3}\right)$ \\
\hline$d$ & $\mathrm{~d}$ & day of the year \\
\hline$d_{0}$ & $\mathrm{~m}$ & zero plane displacement \\
\hline$D_{0}$ & $\mathrm{~m}^{2} \mathrm{~s}^{-1}$ & binary diffusion coefficient for a gas \\
\hline$D_{v}$ & $\mathrm{~m}^{2} \mathrm{~s}^{-1}$ & vapor diffusivity \\
\hline$e_{s}$ & $\mathrm{kPa}$ & saturation vapor pressure \\
\hline$E$ & $\mathrm{~kg} \mathrm{~m}^{-2} \mathrm{~s}^{-1}$ & evaporation rate \\
\hline$g$ & $\mathrm{~m} \mathrm{~s}^{-2}$ & acceleration due to gravity \\
\hline$h$ & & fractional relative humidity \\
\hline$h_{c}$ & $\mathrm{~m}$ & height of crop \\
\hline$h_{s}$ & rad & half day length \\
\hline$H$ & $\mathrm{~J} \mathrm{~m}^{-2} \mathrm{~s}^{-1}$ & sensitive heat flow \\
\hline$J$ & $\mathrm{~W} \mathrm{~m}^{-1}$ & electric energy loss of cable \\
\hline$k$ & & von Karman's constant $(0.41)$ \\
\hline$k_{a}, k_{m}, k_{w}$ & & weighting factor for air, minerals, water \\
\hline$K$ & $\mathrm{~kg} \mathrm{~s} \mathrm{~m} \mathrm{~m}^{-3}$ & hydraulic conductivity \\
\hline$K_{s}$ & $\mathrm{~kg} \mathrm{~s} \mathrm{~m}^{-3}$ & saturated hydraulic conductivity \\
\hline$L$ & $\mathrm{~J} \mathrm{~kg}^{-1}$ & latent heat of vaporization $\left(2.45 \times 10^{6}\right)$ \\
\hline$m$ & & empirical parameter $(2.3)$ \\
\hline$M_{w}$ & $\mathrm{~kg} \mathrm{~mol}{ }^{-1}$ & molecular weight of water $(0.018)$ \\
\hline$P$ & $\mathrm{kPa}$ & barometric pressure \\
\hline $\operatorname{Pr}$ & $\mathrm{kg} \mathrm{m}^{-2}$ & precipitation \\
\hline$q_{\text {cable }}$ & $\mathrm{J} \mathrm{m}^{-2} \mathrm{~s}^{-1}$ & heat flux from surface of cable \\
\hline$q_{h}$ & $\mathrm{~J} \mathrm{~m}^{-2} \mathrm{~s}^{-1}$ & heat flux \\
\hline$q_{w}, q_{v}, q_{v, i}, q_{v, T}$ & $\mathrm{~kg} \mathrm{~m}^{-2} \mathrm{~s}^{-1}$ & water flux, vapor flux (total, isothermal, temperature driven) \\
\hline$r_{c}$ & $\mathrm{~m}$ & radius of cable \\
\hline$r_{h}, r_{v}$ & $\mathrm{~s} \mathrm{~m}^{-1}$ & aerodynamic resistance for heat, for water vapor transfer \\
\hline$r_{s}$ & $\mathrm{~s} \mathrm{~m}^{-1}$ & soil surface resistance for water vapor transfer \\
\hline$R$ & $\mathrm{~J} \mathrm{~mol}^{-1} \mathrm{~K}^{-1}$ & gas constant $(8.31)$ \\
\hline$R_{\text {atm }}, R_{\text {soil }}$ & $\mathrm{W} \mathrm{m} \mathrm{m}^{-2}$ & long-wave radiation from the atmosphere, the soil \\
\hline$R_{\text {meas }}$ & $\mathrm{W} \mathrm{m}^{-2}$ & measured incoming short-wave radiation \\
\hline$R_{\text {meas }, \text { day }}, R_{\text {pot,day }}$ & $\mathrm{J} \mathrm{m}^{-2}$ & measured / potential incoming short-wave radiation during a day \\
\hline$R_{\text {net }}$ & $\mathrm{W} \mathrm{m}^{-2}$ & net radiation across the soil atmosphere interface \\
\hline$R_{\text {sun }}$ & $\mathrm{W} \mathrm{m}^{-2}$ & absorbed short-wave radiation from the sun \\
\hline$s$ & $\mathrm{~kg} \mathrm{~m}{ }^{-3} \mathrm{~K}^{-1}$ & slope of the saturation vapor concentration function \\
\hline$t$ & $\mathrm{~s}$ & time \\
\hline $\begin{array}{l}T, T_{a}, T_{c}, T_{s} \\
T_{t}\end{array}$ & ${ }^{\circ} \mathrm{C}$ & $\begin{array}{l}\text { temperature, in air, at cable, at soil surface } \\
\text { atmospheric transmissivity }\end{array}$ \\
\hline$T_{K}$ & $\mathrm{~K}$ & temperature in Kelvin \\
\hline
\end{tabular}

Table A.6: List of Latin Symbols (A-T) 


\begin{tabular}{lcl}
\hline Symbol & Unit & Description \\
\hline$u$ & $\mathrm{~m} \mathrm{~s}^{-1}$ & wind speed \\
$u^{*}$ & $\mathrm{~m} \mathrm{~s}^{-1}$ & friction velocity \\
$x_{a}, x_{m}$ & $\mathrm{~m}^{3} \mathrm{~m}^{-3}$ & volumetric fraction of air, solid minerals \\
$z$ & $\mathrm{~m}$ & height \\
$z_{r e f}$ & $\mathrm{~m}$ & reference height for data about the atmosphere \\
$z_{H}, z_{M}$ & $\mathrm{~m}$ & surface roughness for heat flux, for momentum flux \\
\hline
\end{tabular}

Table A.7: List of Latin Symbols (U-Z)

\begin{tabular}{|c|c|c|}
\hline Symbol & Unit & Description \\
\hline$\beta$ & & empirical parameter $(0.9)$ \\
\hline$\delta$ & $\mathrm{rad}$ & solar declination \\
\hline$\Delta$ & $\mathrm{kPa} \mathrm{K}^{-1}$ & slope of the saturation vapor pressure curve \\
\hline$\epsilon$ & & parameter for calculation of diffusion \\
\hline$\epsilon_{a c}$ & & atmospheric emissivity depending on cloud cover \\
\hline$\epsilon_{s}$ & & soil emissivity \\
\hline$\underset{\theta}{\lambda}, \lambda_{a}, \lambda_{m}, \lambda_{w}$ & $\mathrm{~W} \mathrm{~m} \mathrm{~m}^{-1} \mathrm{~K}^{-1}$ & thermal conductivity, of air, minerals, water \\
\hline$\theta_{\min }$ & $\%$ & empirical parameter \\
\hline$\theta_{\text {top }}$ & $\%$ & water content in the top $1 \mathrm{~cm}$ layer \\
\hline$\theta_{s}$ & $\mathrm{~m}^{3} \mathrm{~m}^{-3}$ & saturated volumetric water content \\
\hline$\rho_{b}$ & $\mathrm{~kg} \mathrm{~m}^{-3}$ & soil bulk density \\
\hline$\rho_{m}, \rho_{w}$ & $\mathrm{~kg} \mathrm{~m}^{-3}$ & density of soil minerals $\left(2.65 \times 10^{3}\right)$, water $\left(1 \times 10^{3}\right)$ \\
\hline$\sigma$ & $\mathrm{W} \mathrm{m}{ }^{-2} \mathrm{~K}^{-4}$ & StefanBoltzmann constant $\left(5.67 \times 10^{-8}\right)$ \\
\hline$\zeta$ & & stability parameter \\
\hline$\phi$ & $\mathrm{rad}$ & latitude \\
\hline & $\mathrm{m}^{3} \mathrm{~m}^{-3}$ & porosity \\
\hline$\Phi_{H}, \Phi_{M}$ & & atmospheric stability correction factor for heat flux, momentum \\
\hline $\begin{array}{l}\psi \\
\psi_{e}\end{array}$ & $\begin{array}{l}\mathrm{J} \mathrm{kg}^{-1} \\
\mathrm{~J} \mathrm{~kg}^{-1}\end{array}$ & $\begin{array}{l}\text { flux } \\
\text { matric potential } \\
\text { air entry potential }\end{array}$ \\
\hline
\end{tabular}

Table A.8: List of Greek Symbols 


\title{
B Estimation of thermal instabilities in soils for heat dissipa- tion of underground electrical power cables
}

written by Eva Kröner, Gaylon S. Campbell and Marco Bittelli, (Kroener et al., in preparation)

\begin{abstract}
The decentralized production of electric energy from wind requires the installation of more and more underground power cables. From an economic standpoint it becomes very important to estimate and optimize the cables' lifetime which strongly depends on the temperature. A correct estimation of cables' temperature requires an accurate description of the heat balance equation at and around the cable.

Most of the models used to assess heat dissipation from cables, however, do not consider that the soil thermal conductivity strongly depends on soil water content and hydraulic dynamics in the vicinity of the cable. The high temperature around the cable induces a water-vapor-cycle in the soil: liquid water evaporates near the cable and vapor condensates in the more distant and colder parts of the soil. Above a critical heat dissipation rate, the soil around the cable dries out and the water-vapor-cycle breaks.

In this study we propose an analytical estimation for the critical heat dissipation rate as function of soil type, soil temperature and water content. We validate the analytical estimation by comparison to a numerical solution of the coupled heat-water-vapor transport for various soil types and conditions.

The proposed estimation of thermal instabilities in soils can become a powerful tool in the design of underground electrical power cable systems.
\end{abstract}

\section{B.1 Introduction}

Wind energy is an important renewable energy source and has achieved remarkable advances in recent years. Wind technology has significant advanced due to improvements in the design of turbines, generators and structures. In a good windy location, wind power is already competitive with traditional fossil-fuels technologies (Islam et al., 2013). The global wind power installed in the year 2001 was of $238,351 \mathrm{MW}$, with an increase in total installed generating capacity of nearly $75 \%$ over the period of $2005-2011$. Further increase in total capacity is foreseen. 
Because of the distributed nature of energy from each single turbine, wind farms require the installation of underground power cables to transmit the generated energy. An optimally sized cable results in minimum cost and high reliability. Wind and solar power plants, in particular, strive to optimize cable design. There are many causes of cable's deterioration: thermal (expansion, contraction, melting/flow of insulation, chemical reaction), electrical (electrical/water treeing, intrinsic breakdown), mechanical (yielding, cracking, rupture) and environmental (corrosion). In general, as time passes, the cables' insulation weakens, which increases the potential for a line fault. If the cables are installed and designed properly, this debilitating process can take years and might be avoided.

One of the most important causes of cable's deterioration is the thermal deterioration. Electric losses in the cables result in thermal energy that dissipates into the environment through the soil. The cable temperature, for a given rate of heat production, is determined by the thermal conductivity of the soil, the temperature of the environment, and the geometry of the path between the cable and the environment. Many analytical (Neher and McGrath, Oct. 1957; Papagiannopoulos et al., 2013; Chatziathanasiou et al., 2013; de Lieto Vollaro et al., 2011b) and numerical (Ocłoń et al., 2015a b; Kovac et al., 2013, Canova et al., 2012; de Lieto Vollaro et al., 2011a; De Leon and Anders, 2008) researches have been performed and presented, to study the heat dissipation of buried electric cables. In these studies, however, it was not considered that heat dissipation strongly depends on hydraulic dynamics and water content distribution in soil (Taylor and Cavazza, 1954; de Vries, 1963).

Numerical models that account for coupled heat, water and vapor flow such as the one presented by Kroener et al. (2014a) are powerful tools to better design cable installations. Moreover, they allow for choosing the correct cable specifications, installation depth and backfill. However, multi-dimensional numerical solutions are time consuming, they require knowledge of numerical methods, and they require a detailed parameterization of the model. Therefore simpler analytical solutions, that still preserve the fundamental driving forces of the system, are useful for decision making.

The soil thermal conductivity is strongly dependent on the water content of the soil. Heat from cables tends to dry the soil around it, thus decreasing the thermal conductivity of the soil and increasing the cable temperature. A soil, in which this phenomena occurs, is defined as thermally unstable. If the soil around the cable ultimately will completely dry out due to cable's heating, heat dissipation will be highly reduced facilitating cable's damage. On the other hand, if it can be guaranteed that the soil will stay wet, heat dissipation will be 
facilitated leading to cable's preservation. Since soil thermal conductivity depends on soil water content, these conditions must be determine for a correct cable's design. For instance, cable's cost depends on the cable's resistance to temperature increase. If the analysis suggests that under given conditions the cable may experience high temperatures because of thermal instability, it may be necessary to select a cable that can operate at higher temperatures without failure.

The purpose of this paper is: a) to present a simplified analysis to demonstrate the soil conditions under which thermal stability occurs, b) to derive, based on the simplified analysis, an analytical solution for the critical heat dissipation rate and c) to test the proposed solution against experimental data and numerical studies.

\section{B.2 Governing equations}

Analyzing coupled transport of heat and water in soil can be complex (Hartley and Black, 1981; Bittelli et al., 2008). Thermal energy flux in soil is the sum of sensible heat flux, through thermal conduction in soil, $q_{\mathrm{hc}}$ and latent heat flux $q_{\mathrm{hL}}$ transported by water vapor. Total water flux in soil is composed of liquid water flux $q_{\mathrm{lw}}$, temperature driven vapor flux $q_{\mathrm{vT}}$ and vapor flux driven by water potential $q_{\mathrm{vP}}$.

Fig. B.1 illustrates the contribution of the different fluxes to the energy and mass budget in wet and dry soil: (a) under wet soil conditions a stable liquid water-water vapor cycle is established, where liquid water flow toward the cable is in equilibrium with vapor flow away from the cable, the latter carrying out a considerable part of thermal energy away from the cable as latent heat, (b) under dry soil conditions the soil hydraulic conductivity is very low and the contribution of the liquid water flow almost disappears, therefore also reducing the dissipation of latent heat away from the cable. Under condition (b), temperature driven vapor flow equilibrates with vapor flow driven by water potential. The net water vapor flow becomes zero, and the thermal energy can no longer dissipate in form of latent heat, instead all heat dissipates by thermal conduction in the soil and the cable temperature is controlled by the low, dry soil thermal conductivity.

Here we provide a short summary of the main equations employed to describe coupled heat, liquid water and vapor flow. For more detailed informations we refer to Bittelli et al. (2008) were it was tested against experimental data of heat, water and vapor flow dynamics in soils. Kroener et al. (2014a) have used this model to describe heat dissipation from underground electrical power cables. They tested the model with data from a down-scaled 
a) wet soil conditions

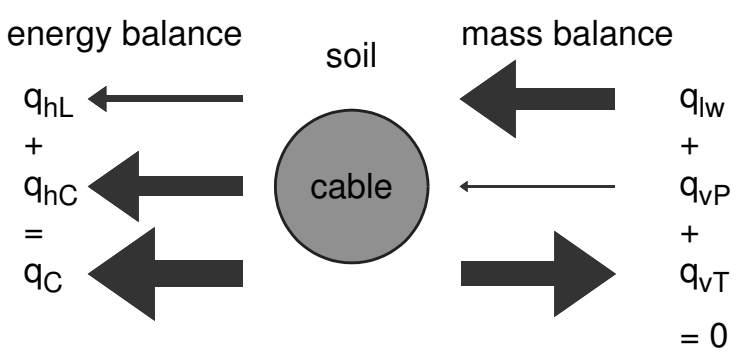

b) dry soil conditions

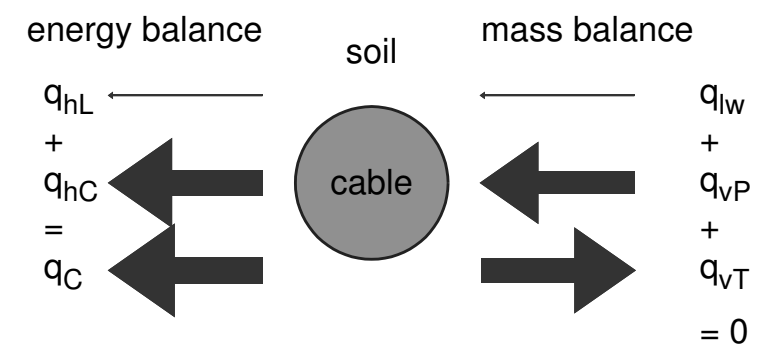

Figure B.1: Energy and mass balance for heat dissipation in a) wet soil and b) dry soil. The thickness of the arrows indicates the quantitative contribution of latent heat flux $q_{\mathrm{hL}}$, sensitive heat flux $q_{\mathrm{hC}}$, heat dissipation from the cable $q_{\mathrm{C}}$, water vapor flux driven by a gradient in water potential $q_{\mathrm{vP}}$, liquid water flux $q_{\mathrm{lw}}$, and water vapor flux driven by a gradient in temperature $q_{\mathrm{vT}}$ to the overall energy and mass budget. 
experiment of heat dissipation from electrical underground cables de Lieto Vollaro et al. 2014).

Richards equation describes liquid water flow in soils. Neglecting gravity, and for a radial geometry of the cable's environment it is formulated as:

$$
\frac{q_{\mathrm{lw}}}{2 \pi r}=-K \frac{\mathrm{d} \psi}{\mathrm{d} r}
$$

where $r$ is the radial coordinate, $q_{\mathrm{lw}}$ is flux of water to the cable per unit length of cable $\left(\mathrm{kg} \mathrm{s}^{-1} \mathrm{~m}^{-1}\right), K$ is hydraulic conductivity of the soil $\left(\mathrm{kg} \mathrm{s} \mathrm{m}^{-3}\right)$ and $\psi$ is the matric potential of the soil $\left(\mathrm{J} \mathrm{kg}^{-1}\right)$.

Here we neglect the contribution of temperature driven liquid flow which is generally much smaller than liquid flow driven by the water potential gradient.

The hydraulic conductivity can be expressed as:

$$
K= \begin{cases}K_{s}\left(\frac{\psi_{e}}{\psi}\right)^{n} & \text { if }\left(\psi<\psi_{e}\right) \\ K_{s} & \text { if }\left(\psi \geq \psi_{e}\right)\end{cases}
$$

where $n$ is a constant ranging from 2 to 3.5. The saturated conductivity $K_{s}$ and the air entry matric potential $\psi_{e}$, as well as $n$ depend on soil texture and bulk density (Bittelli et al. 2015)

Fick's first law governs steady vapor transport away from the cable. We can write

$$
\frac{q_{\mathrm{v}}}{2 \pi r}=-D \frac{\mathrm{d} c}{\mathrm{~d} r}
$$

where $c$ is the vapor concentration $\left(\mathrm{kg} \mathrm{m}^{-3}\right)$ and $D$ is the vapor diffusivity in soil. The vapor diffusivity in soil is (Campbell, 1985):

$$
D=D_{0} \beta \phi^{m}
$$

where $\phi$ is the air filled porosity of the soil and $D_{0}$ is the binary diffusion coefficient of water vapor in air $D_{0}(T, P)\left(\mathrm{m}^{2} \mathrm{~s}^{-1}\right) . \beta=0.9$ and $m=2.3$ are constants. The air filled porosity is:

$$
\phi=\theta_{s}-\theta=\theta_{s}\left[1-\left(\psi_{e} / \psi\right)^{1 / b}\right]
$$

where $\theta_{s}$ is the saturation water content of the soil and $b$ a parameter that depends on the soil texture. 
Since the soil vapor concentration depends on water potential and temperature, we can expand Eq. (B.3) to express vapor flow as the sum of a water potential driven flux component $q_{\mathrm{vP}}$ and a temperature driven flux component $q_{\mathrm{vT}}$ :

$$
\frac{q_{\mathrm{v}}}{2 \pi r}=\frac{q_{\mathrm{vP}}}{2 \pi r}+\frac{q_{\mathrm{vT}}}{2 \pi r}=-D h c_{v}^{\prime} \frac{M_{w}}{R T_{K}} \frac{\mathrm{d} \psi}{\mathrm{d} r}-D h s \frac{\mathrm{d} T}{\mathrm{~d} r}
$$

where $h=\exp \left(M_{w} \psi / R T_{K}\right)$ is relative humidity, $c_{v}^{\prime}$ is saturated vapor concentration, $M_{w}$ is the molecular mass of water, $R$ is the universal gas constant, $T_{K}$ is the soil Kelvin temperature, and $s$ is the slope $\mathrm{d} c_{v}^{\prime} / \mathrm{d} T$ of saturation vapor concentration function.

Conductive heat flux is driven by a gradient in temperature and proportional to the soil thermal conductivity $\lambda$ :

$$
\frac{q_{\mathrm{hC}}}{2 \pi r}=-\lambda \frac{\mathrm{d} T}{\mathrm{~d} r}
$$

Latent heat flux $q_{\mathrm{hL}}$ is proportional to vapor flux and the latent heat of vaporization $L$ :

$$
q_{\mathrm{hL}}=L q_{\mathrm{v}}
$$

Continuity for mass states that the sum of water and vapor flow from the cable is zero and continuity for energy states that sum of sensible and latent heat flow from the cable are equal to the heat produced by the cable $q_{\mathrm{C}}$. Eqs. B.1, B.6, B.7 and B.8 yield to the coupled system of equations for steady state heat dissipation from the cable:

$$
\begin{aligned}
0 & =K \frac{\mathrm{d} \psi}{\mathrm{d} r}+D h c_{v}^{\prime} \frac{M_{w}}{R T_{K}} \frac{\mathrm{d} \psi}{\mathrm{d} r}+D h s \frac{\mathrm{d} T}{\mathrm{~d} r} \\
\frac{q_{\mathrm{c}}}{2 \pi r} & =-\lambda \frac{\mathrm{d} T}{\mathrm{~d} r}-L D h c_{v}^{\prime} \frac{M_{w}}{R T_{K}} \frac{\mathrm{d} \psi}{\mathrm{d} r}-L D h s \frac{\mathrm{d} T}{\mathrm{~d} r}
\end{aligned}
$$

\section{B.3 Order of magnitude estimation}

In the simplified analysis we assume that water movement away from the cable is entirely in the vapor phase and entirely driven by a temperature gradient, and that water flow back toward the cable is entirely in the liquid phase and entirely driven by a matric potential gradient. Condensation of vapor into liquid water leads to a continuous decrease of water and vapor flow with increasing distance from the cable. In this order of magnitude estimation, however, we assume that liquid water $q_{\mathrm{lw}}$ and water vapor flow $q_{\mathrm{v}}$ do not decrease with increasing distance from the cable. 
Table B.1: Hydraulic properties of soils as a function of soil texture

\begin{tabular}{lcccccc}
\hline Texture & Silt & Clay & $\begin{array}{c}\psi_{e} \\
\mathrm{~J} \mathrm{~kg}^{-1}\end{array}$ & $b$ & $\begin{array}{c}K_{s} \\
\mathrm{~kg} \mathrm{~s} \mathrm{~m}^{-3}\end{array}$ & $\theta_{s}$ \\
\hline sand & 0.05 & 0.03 & 0.7 & 1.7 & $5.8 \times 10^{-3}$ & 0.37 \\
loamy sand & 0.12 & 0.07 & 0.9 & 2.1 & $1.7 \times 10^{-3}$ & 0.38 \\
sandy loam & 0.25 & 0.10 & 1.5 & 3.1 & $7.2 \times 10^{-4}$ & 0.41 \\
loam & 0.40 & 0.18 & 1.1 & 4.5 & $3.7 \times 10^{-4}$ & 0.43 \\
silt loam & 0.65 & 0.15 & 2.1 & 4.7 & $1.9 \times 10^{-4}$ & 0.44 \\
sandy clay loam & 0.13 & 0.27 & 2.8 & 4.0 & $1.2 \times 10^{-4}$ & 0.41 \\
clay loam & 0.34 & 0.34 & 2.6 & 5.2 & $6.4 \times 10^{-5}$ & 0.45 \\
silty clay loam & 0.58 & 0.33 & 3.3 & 6.6 & $4.2 \times 10^{-5}$ & 0.46 \\
sandy clay & 0.07 & 0.40 & 2.9 & 6.0 & $3.3 \times 10^{-5}$ & 0.44 \\
silty clay & 0.45 & 0.45 & 3.4 & 7.9 & $2.5 \times 10^{-5}$ & 0.51 \\
clay & 0.20 & 0.60 & 3.7 & 7.6 & $1.7 \times 10^{-5}$ & 0.50 \\
\hline
\end{tabular}

Under the assumption of constant liquid water flow, the hydraulic process is similar to water flow to a plant root, which was analyzed by Cowan (1965): the unsaturated hydraulic conductivity function of soil is such that there is a limiting rate of water flow for any given bulk soil water content or potential. If the rate of vapor flow from the cable is greater than this limiting rate of liquid return flow the soil is thermally unstable and will dry out.

\section{B.3.1 Limiting rate of liquid water flow toward the cable}

Combining Eq. (B.1) and Eq. (B.2) and integrating from the cable surface at $r_{c}$ to the bulk soil at $r_{s}$ gives

$$
\frac{q_{\mathrm{lw}}}{2 \pi} \ln \left(\frac{r_{c}}{r_{s}}\right)=\frac{K_{s}\left|\psi_{e}\right|^{n}}{1-n}\left(\left|\psi_{s}\right|^{1-n}-\left|\psi_{c}\right|^{1-n}\right)
$$

where $\psi_{s}$ and $\psi_{c}$ are the water potential at the soil and cable surface respectively, while $|\cdot|$ indicates the absolute value. The maximum value for water flow occurs when the absolute value of the water potential at the cable surface goes to infinity (very dry) and the term $\left|\psi_{c}\right|^{1-n}$ in Eq. (B.11) becomes zero. We can therefore write the maximum flux per unit length of cable $\left(\mathrm{kg} \mathrm{m}^{-1} \mathrm{~s}^{-1}\right)$ as

$$
q_{\mathrm{lw}}^{\lim }=\frac{2 \pi K_{s}\left|\psi_{e}\right|^{n}\left|\psi_{s}\right|^{1-n}}{(n-1) \ln \left(r_{s} / r_{c}\right)}
$$

To evaluate $q_{\mathrm{lw}}^{\text {lim }}$ we need air entry conductivity and water potentials for representative soils. For typical soil types Campbell and Norman (1998) give the values shown in Tab. B.1.

The value for $n$ is computed as $n=2+3 / b$ (Campbell, 1985). Assuming that the bulk soil values are measured at about 3 times the radius of the cable, the term $\ln \left(r_{s} / r_{c}\right)$ has a value around 1. The results we obtained are not very sensitive to this assumption, 


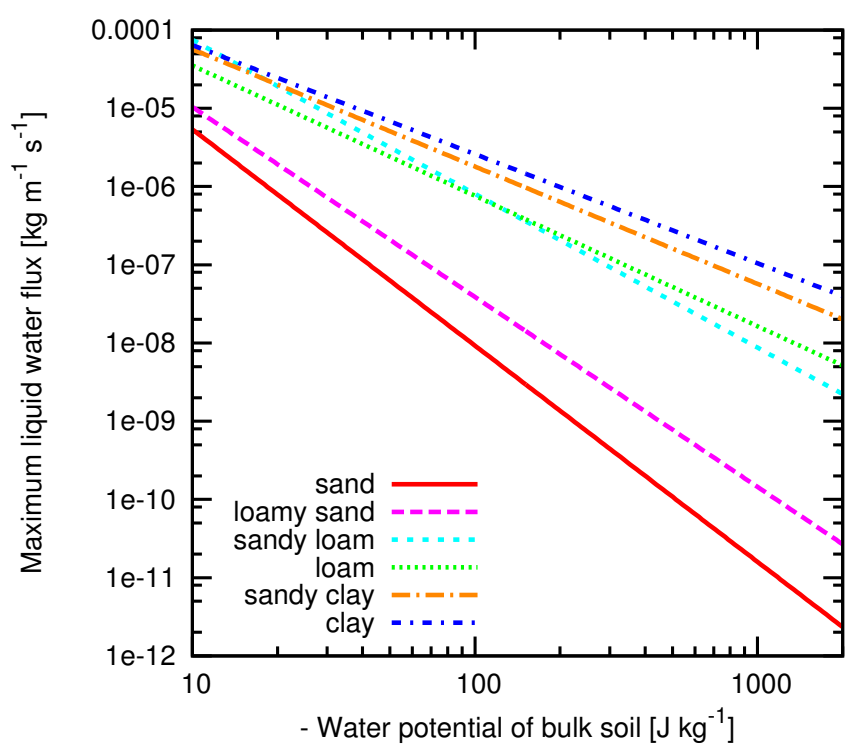

Figure B.2: Limiting water flux $q_{\mathrm{lw}}^{\text {lim }}$ as function of bulk soil water potential (Eq. B.12 for typical soil types using the parameters of Tab. B.1

but measurements have shown that the drying effect only influences the soil close to the cable, so this seems like a reasonable value to use. Fig. B.2 shows the limiting flux $q_{\mathrm{lw}}^{\text {lim }}$ for various soil types. For instance at a bulk soil water potential of $\psi_{s}=-100 \mathrm{~J} \mathrm{~kg}^{-1}$ the maximum liquid water flow toward a cable in a sandy soil is ca. $10^{-8} \mathrm{~kg} \mathrm{~m}^{-1} \mathrm{~s}^{-1}$. Overall, although there is soil property variability within a textural class, Tab. B.1 is very useful for practical purposes. Underground cables are installed over many kilometers of land, with variable textural properties along the buried line, making textural analysis expensive and time consuming. The use of geological and soil maps where texture is commonly available together with this table allows for acquisition of the required parameters.

\section{B.3.2 Vapor flow away from the cable}

Using the assumption that vapor flow is driven by a temperature gradient only, Eq. B.6 gives:

$$
q_{v}=-2 \pi r D s h \frac{\mathrm{d} T}{\mathrm{~d} r}
$$

The humidity in soil is usually near one and therefore we assume $h=1$.

Solving Eq. B.7) for the temperature gradient and substituting it into Eq. B.13) gives a relation between vapor flow $q_{\mathrm{v}}$ and conductive heat flow $q_{\mathrm{hC}}$ : 


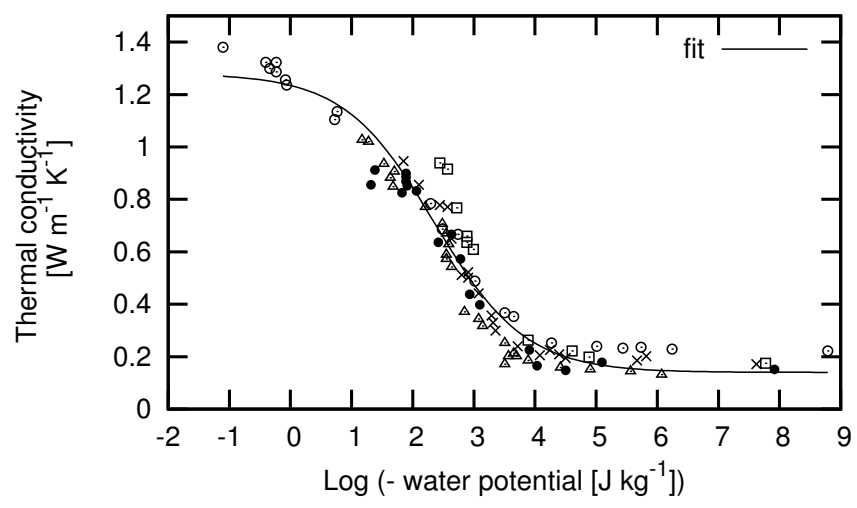

Figure B.3: Thermal conductivity as function of water potential (Eq. B.16) obtained as best fit to the data set from McInnes (1981).

$$
q_{\mathrm{v}}=\frac{s D}{\lambda} q_{\mathrm{hC}}
$$

Using Eq. (B.4) for the vapor diffusivity and the assumption that the heat from the cable dissipates completely by thermal conductivity $\left(q_{\mathrm{C}}=q_{\mathrm{hC}}\right)$ we obtain the vapor flow as function of energy loss at the cable:

$$
q_{\mathrm{v}}=\frac{s D_{0} \beta \phi^{m}}{\lambda} q_{\mathrm{C}}
$$

To determine $q_{\mathrm{v}}$ we need values for $\lambda, \phi, s, D_{0}$ and $q_{\mathrm{C}}$. For this simplified analysis we consider these quantities as being constant in space, dependent only of bulk soil water potential and temperature and independent of the decrease of water potential and increase of soil temperature towards the cable. Based on the thermal conductivity data of five soil types ranging from fine sand to silt loam (McInnes, 1981), Campbell (1988) showed that the thermal conductivity of soil can be approximated as a function of water potential and independent of texture. We obtained the best fit to their data with the following parametrization (Fig. B.3):

$$
\lambda=1.28-\frac{1.14}{1+(-\psi / 227)^{-0.588}}
$$

The slope $s$ of the saturation vapor concentration versus temperature function has a value of $0.001 \mathrm{~kg} \mathrm{~m}^{-3} \mathrm{~K}^{-1}$ at $20^{\circ} \mathrm{C}$ and $0.004 \mathrm{~kg} \mathrm{~m}^{-3} \mathrm{~K}^{-1}$ at $50{ }^{\circ} \mathrm{C}$ (Campbell, 1985). We used the larger value for our calculations, assuming the cable would be above ambient temperature. The diffusivity of vapor in air has a weak temperature dependence. We used $D_{0}=2.9 \times 10^{-5}$ $\mathrm{m}^{2} \mathrm{~s}^{-1}$, which is the value at $50{ }^{\circ} \mathrm{C}$. 


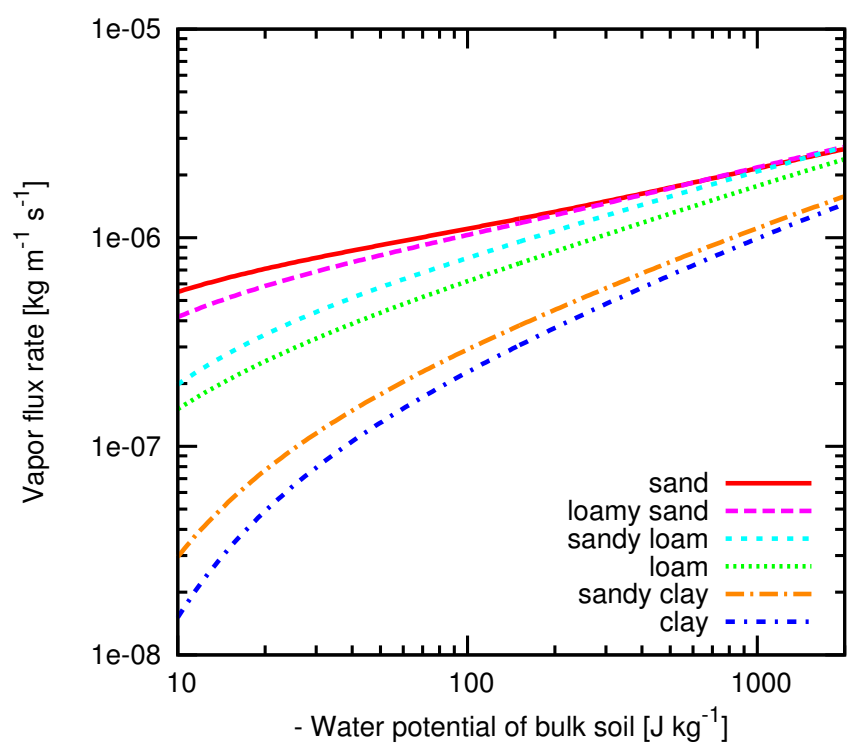

Figure B.4: Vapor flux $q_{\mathrm{v}}$ as function of bulk soil water potential (Eq. B.15) for typical soil types (Tab. B.1) assuming a cable heat dissipation rate of $q_{\mathrm{C}}=100 \mathrm{~W} \mathrm{~m}^{-1}$.

Fig. B.4 shows the vapor flow from the cable as function of soil water potential (Eq. (B.15) for these values and a heat production at the cable of $q_{\mathrm{C}}=100 \mathrm{~W} \mathrm{~m}^{-1}$.

\section{B.3.3 Critical heat dissipation rate}

At the critical heat dissipation rate where the soil begins to be thermally unstable, liquid water flow $q_{\mathrm{lw}}$ toward the cable and vapor flow from the cable $q_{\mathrm{v}}$ are equal. Combining Eqs. B.12 and (B.15) provides a relation between bulk soil water potential and critical heat dissipation rate $q_{\mathrm{C}}^{c r}$ from the cable at the threshold of stable thermal soil conditions:

$$
q_{\mathrm{C}}^{c r}=\frac{2 \pi K_{s} \psi_{e}^{n} \psi_{s}^{1-n} \lambda}{(n-1) \ln \left(r_{s} / r_{c}\right) s D_{0} \beta \phi^{m}}
$$

\section{B.4 Numerical simulation}

To perform a comparison between the analytical solution presented above and a numerical solution, the coupled system of heat dissipation (Eqs. $B .9$ and $(B .10)$ ) was numerically solved, without the simplifications and assumptions used in the analytical solution (order of magnitude estimation). The numerical solution accounts for vapor condensation in the soil, leading to a decrease in water flow rate $q_{\mathrm{lw}}$ and vapor flow rate $q_{\mathrm{v}}$, with increasing distance to the cable. 


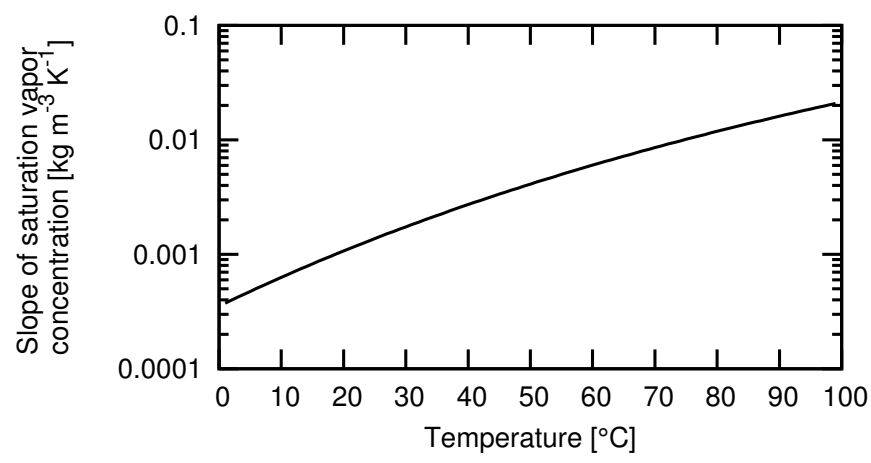

Figure B.5: Slope of saturation vapor concentration (Eq. B.20).

\section{B.4.1 Parameter functions}

Humidity is now expressed as function of water potential:

$$
h=\exp \left(\frac{M_{w} \psi}{R T_{K}}\right)
$$

where $M_{w}$ is the molecular mass of water, $T_{K}$ the Kelvin temperature and $R$ the gas constant. Saturation vapor concentration is derived from Buck (1981):

$$
c_{v}^{\prime}=0.611 \exp \left(\frac{17.27 T}{T+237.3}\right) \frac{M_{w}}{R T_{K}}
$$

Multiplication by $M_{w} /\left(R T_{K}\right)$ is necessary to convert pressure [kPa] to concentration $[\mathrm{kg}$ $\mathrm{m}^{-3}$. The slope of saturation vapor concentration (Fig. B.5) is:

$$
s=\frac{4098 \cdot c_{v}^{\prime}}{(T+237.3)^{2}}
$$

The binary diffusion coefficient of water vapor in air is:

$$
D_{0}\left(T_{K}, P\right)=D_{0}(273.15 \mathrm{~K}, 101.3 \mathrm{kPa})\left(\frac{T_{K}}{273.15 \mathrm{~K}}\right)^{1.75}\left(\frac{101.3 \mathrm{kPa}}{P}\right)
$$

with the binary diffusion coefficient for standard conditions: $D_{0}(273.15 \mathrm{~K}, 101.3 \mathrm{kPa})=$ $2.12 \times 10^{-5} \mathrm{~m}^{2} \mathrm{~s}^{-1}$. We used the apparent thermal conductivity model from Campbell et al. (1994) - it originates in the theory of de Vries (1963) - to express the soil thermal conductivity $\lambda$ as a weighted sum of the components' conductivities, $\lambda_{w}$ (water), $\lambda_{a}$ (air) and $\lambda_{m}$ (minerals):

$$
\lambda=-\frac{k_{w} \theta \lambda_{w}+k_{a} x_{a} \lambda_{a}+k_{m} x_{m} \lambda_{m}}{k_{w} \theta+k_{a} x_{a}+k_{m} x_{m}}
$$


where $\theta, x_{a}$ and $x_{m}$ are the components' volume fractions and $k_{w}, k_{a}$ and $k_{m}$ are additional weighting factors. For further information on how to calculate these weighting factors we refer to Bittelli et al. (2015).

\section{B.4.2 Boundary conditions}

The boundary conditions at the cable surface at $r_{c}=0.025 \mathrm{~m}$ are no-flow for water and a thermal energy flow of $q_{C}=J$, where $J\left[\mathrm{~W} \mathrm{~m}^{-1}\right]$ is average electric energy loss of the cable. The boundary conditions in the bulk soil at $r_{s}=3 r_{c}=0.075 \mathrm{~m}$ is a constant temperature of $T_{s}=20^{\circ}$ and a constant water potential of $\psi_{s}$. These conditions have also been chosen in the entire domain as an initial guess for the Newton solver.

\section{B.4.3 Critical bulk water potential}

To determine the critical water potential for a given cable heat dissipation rate, the system of equations is numerically solved several times with decreasing water potential stepwise as a bulk soil condition until the critical bulk water potential is reached. The definition of the critical point, however, is not obvious: with decreasing bulk water potential the hydraulic conductivity $K$ decreases and the ratio between liquid water $q_{\mathrm{lw}}$ and potential driven vapor flow $q_{\mathrm{vP}}$ becomes smaller. We defined the critical water potential as the point where liquid water and water potential driven vapor flow have the same value

$$
q_{\mathrm{lw}}\left(r=r_{c}\right)=q_{\mathrm{vP}}\left(r=r_{c}\right)
$$

Note that mass balance $q_{\mathrm{lw}}+q_{\mathrm{vP}}+q_{\mathrm{vT}}=0$ requires $q_{\mathrm{lw}}\left(r=r_{c}\right)=q_{\mathrm{vP}}\left(r=r_{c}\right)=$ $-1 / 2 q_{\mathrm{vT}}\left(r=r_{c}\right)$.

\section{B.4.4 Implementation}

The stationary radial problem (Eqs. (B.9) and (B.10) has been solved using a one dimensional grid with 100 elements. The element size is geometrically increasing from $8 \cdot 10^{-9}$ $\mathrm{m}$ at the cable surface to $7 \cdot 10^{-4} \mathrm{~m}$ in the bulk soil. The non-linear equations have been solved with a Newton method and the linear equations with the Thomas-Algorithm. The Python-based program is available online at http://www.dista.unibo.it/ bittelli/. 


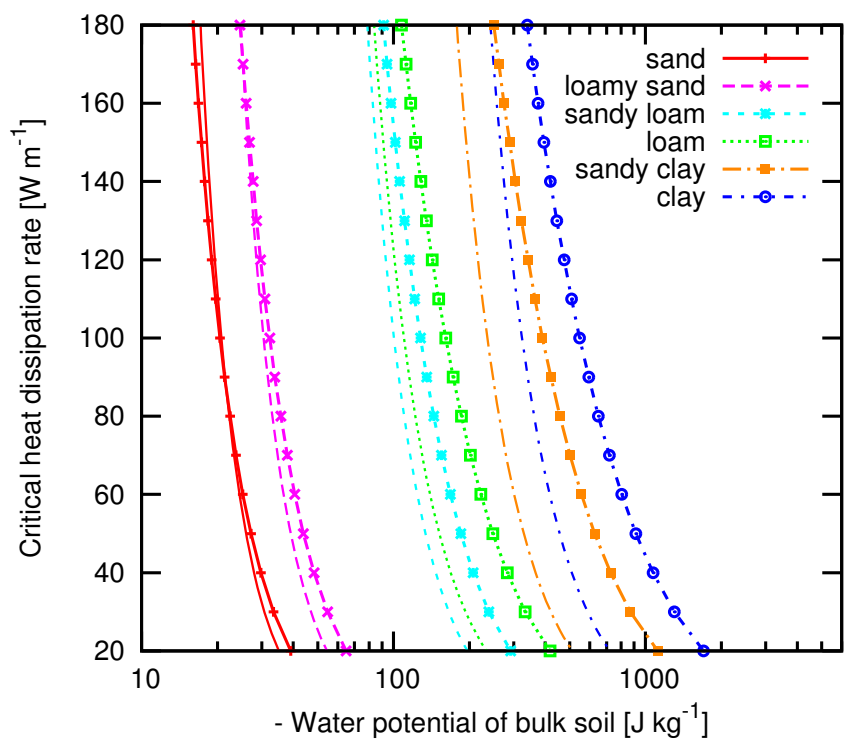

Figure B.6: Relation between bulk soil water potential and cable heat dissipation rate for various soil types (Tab. B.1) at the critical point where soil turns thermally unstable. The results of the numerical simulations are illustrated as linespoints. The lines show the analytical estimations (Eq. B.17).

\section{B.5 Results}

According to Hartley and Black (1981) the practical range of heat generation from electrical power cables is between 20 and $180 \mathrm{~W} \mathrm{~m}^{-1}$. Within this range, Fig. B.6 shows the critical heat dissipation rate $q_{\mathrm{C}}^{\text {cr }}$ for various soil textures as function of bulk soil water potential. Both analytical estimation (Eq. B.17) as well as numerical solution are plotted. The critical bulk soil water potentials for a sandy soil are in the range from $-15 \mathrm{~J} \mathrm{~kg}^{-1}$ to $-40 \mathrm{~J} \mathrm{~kg}^{-1}$ and for a clay soil in the range from $-330 \mathrm{~J} \mathrm{~kg}^{-1}$ to $-1700 \mathrm{~J} \mathrm{~kg}^{-1}$. For coarser soils the estimated critical heat dissipation rate fits very well with the numerical simulations. In finer soils, however, the critical bulk soil water potential determined numerically is lower than the one predicted by Eq. B.17.

The differences between analytical estimation and numerical solution for the finer textured soils can be explained by the simplifactions of the analytical solution: (a) the simplified approximation of the thermal conductivity (Eq. (B.16), (b) the assumption that $s, D, h$ and $\lambda$ do not change with decreasing water potential near the cable but depend only on bulk soil water potential and (c) that there is no vapor flow driven by a gradient in water potential. Indeed when changing the points (a), (b) and (c) in the numerical program the result is the 
same as the analytical expectation.

For a sandy soil under wet conditions hydraulic conductivity is very high compard to vapor diffusivity but then the hydraulic conductivity decreases strongly with decreasing water potentail. For a clay soil, however, the hydraulic conductivity is low under wet conditions and then decreasese slowly with decreasing water potential. The range of matric potential where vapor diffusivity and hydraulic conductivity are of same magnitude is larger for a fine textured soil. This explains why the deviation between analytical and numerical solution in Fig. B.6 is bigger for the finer soils than for the coarse textured soils.

For a sand and a clay the radial solution of temperature, water potential, and water content at the critical point, where soil turns thermally unstable, are shown in Fig. B.7 for heat dissipation rates of $q_{\mathrm{C}}=20,80$ and $100 \mathrm{~W} \mathrm{~m}^{-1}$.

Fig. B.7 shows that the temperature at the critical heat dissipation rate is higher in sandy soils $\left(29-64^{\circ} \mathrm{C}\right)$ than in clay soils $\left(25-55^{\circ} \mathrm{C}\right)$. This can be understood when looking at the hydraulic condition: the critical water potentials are less negative in sandy soils, but the bigger pores of a sandy soil drain fast and therefore the soil around the cable is much drier for a sandy soil $(\theta=0.01-0.03)$ than for a clay soil $(\theta=0.18-0.21)$. The dryer sandy soil has a lower thermal conductivity and therefore the temperatures at the cable surface are higher compared to those in a clay soil. Temperature and soil water content at the cable surface at the critical point where the soil turns thermally unstable can be seen in Fig. B.8 as function of cable heat dissipation rate for various soil types. One should consider that this temperature depends on the location of the outer boundary $r_{s}$. The larger $r_{s}$, the larger the temperature at the cable surface.

Fig. B.9 shows conductive heat flow, temperature driven vapor flow, water flow, and water potential driven vapor flow at the critical bulk soil water potential where thermal instabilities occur at the cable surface.

For both, the sandy soil as well as for the clay soil, the conductive heat flow $q_{\mathrm{hC}}$ is almost the same as the cable heat dissipation rate $q_{\mathrm{C}}$, except for small deviations in the vicinity of the cable due to latent heat transport. Water flow toward the cable and temperature driven vapor flow away from the cable almost equilibrate, only under dry conditions near the cable surface the contribution of potential driven vapor flow becomes considerable in the total mass balance.

While the potential driven vapor flow explains the differences between water flow $q_{\mathrm{lw}}$ and temperature driven vapor flow $q_{\mathrm{vT}}$ in the immediate vicinity of the cable, it cannot explain 


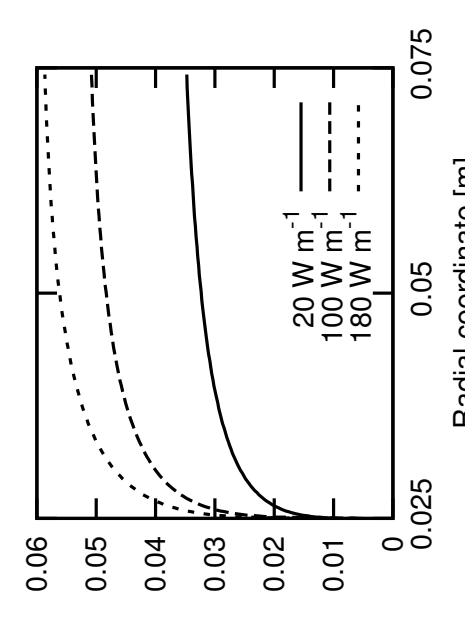

$\left[{ }_{\varepsilon} w_{\varepsilon} m\right]$

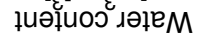

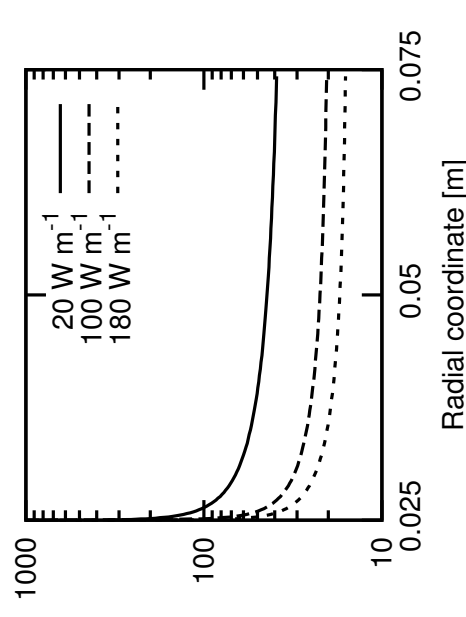

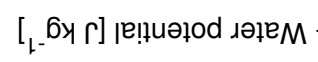

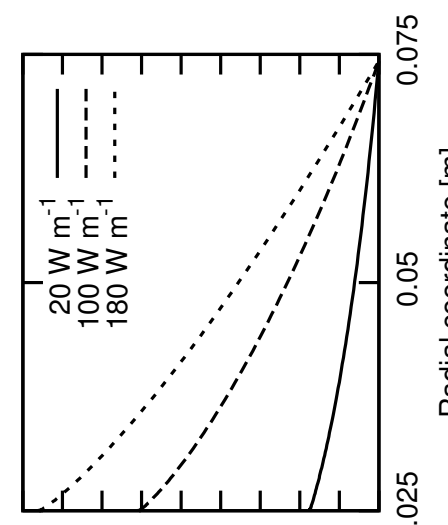

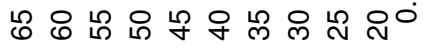

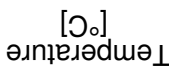

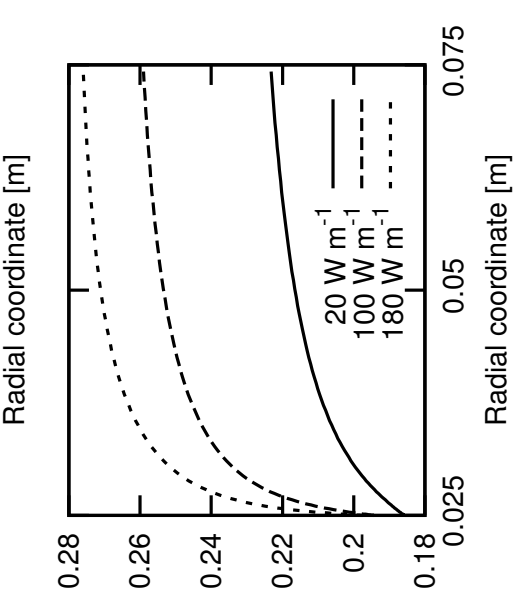

$\left[\begin{array}{ll}L_{-} u & w]\end{array}\right.$

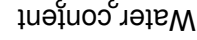

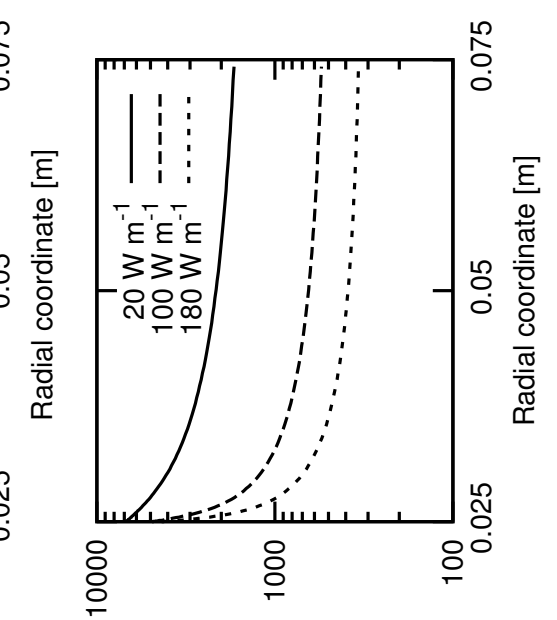

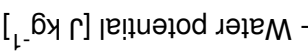

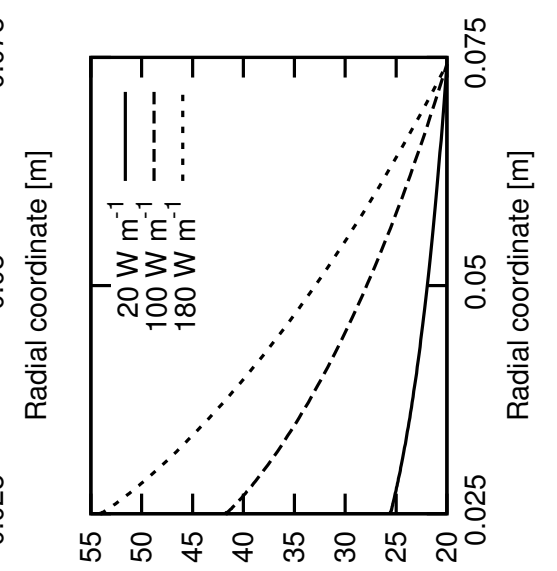

[O०]

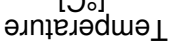

:

$\underset{\square}{\square} \overline{0}$

๘

ส્ઞ

눙

궁

疍

$\approx$ ठี

శี

官

")

苞

क

के

$\overrightarrow{7}$

牚

ن.

范

ซี

.డ్తే స్త్ర

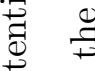

ฉ. .

¿

营

ब

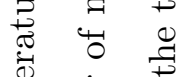

峁 苛

वे

崩苞 苞

范

总莺

용

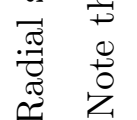

步

$\begin{array}{lll}\approx & 0 \\ \ddot{\sim} & \ddot{0} & 0\end{array}$

苘 $\ddot{0}$

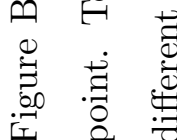



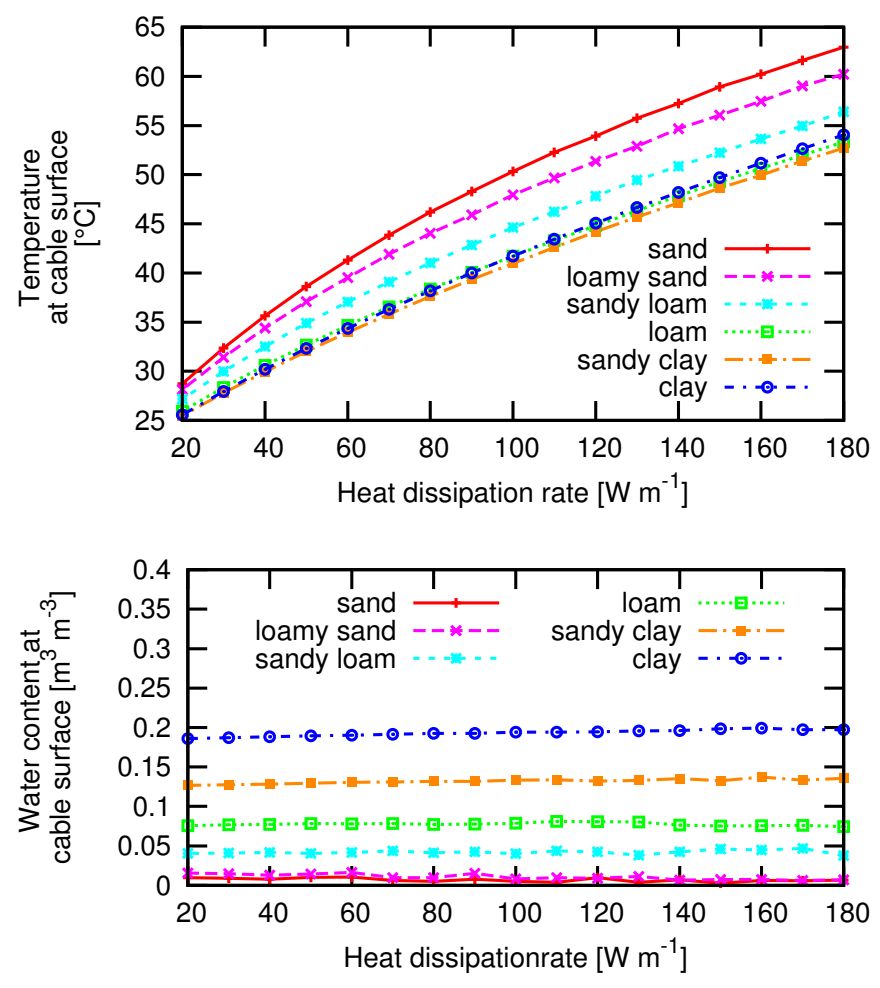

Figure B.8: Soil temperature and soil water content at the cable surface as function of heat dissipation rate at the critical point where soil turn thermally unstable. Note that the bulk soil water potentials vary among the different solutions as shown in Fig. B.6 
why both, water flow as well as temperature driven vapor flow, decrease with increasing distance from the cable. This, however, becomes clear when looking at Eq. B.15): $s$ and $\phi$ decrease with decreasing absolute water potential and decreasing temperature while $\lambda$ increases with decreasing absolute water potential.

Since the conductive heat $q_{\mathrm{hC}}$ is almost constant (Fig. B.9) this means that the vapor flow away from the cable $q_{\mathrm{vT}}$ decreases with increasing distance to the cable. The condensed vapor results in return flow of liquid water toward the cable. To guarantee mass balance also the water flow toward the cable $q_{\mathrm{lw}}$ has to decrease with increasing distance from the cable as shown in Fig. B.9. Note that in the order of magnitude estimation $s, \phi$ and $\lambda$ are evaluated as function of bulk soil water potential and bulk soil temperature only. They are constant in space and as a result $q_{\mathrm{v}}$ is constant in space when determined using Eq. (B.15). The order of magnitude estimation does not consider condensation of vapor in the colder parts of the soil.

Fig. B.10 shows solutions for a sandy soil beyond the critical point where the liquid water - water vapor cycle no longer reaches to the cable surface. Instead the soil around the cable is dry and the hydraulic conductivity becomes so low that liquid water flow can no longer equilibrate the temperature driven vapor flow. Instead the steep decrease in water potential results in a larger potential driven vapor flow. Now $q_{\mathrm{vT}}$ and $q_{\mathrm{vP}}$ equilibrate around the cable so that the net vapor flow $q_{\mathrm{v}}$ becomes negligible. The water-vapor cycle starts now in the wetter region of the soil, compare distribution of water content and water flow.

Fig. B.11 shows that evaporation occurs in a very narrow region where the soil starts to become wet enough so that water flow can sustain the evaporation rate. Condensation instead occurs within a wide region after the narrow region of evaporation. The condensation rate decreases with increasing distance from the cable. Calculations (not shown here) revealed that in clay soils the evaporation occurs in a broader region than in a sandy soil.

\section{B.6 Discussion}

For sand, at a cable heat dissipation rate of $180 \mathrm{~W} / \mathrm{m}$, the critical water potential is around $-10 \mathrm{~J} / \mathrm{kg}$, and at $20 \mathrm{~W} / \mathrm{m}$ it is around $-50 \mathrm{~J} / \mathrm{kg}$. For the clay the critical water potentials are around $-300 \mathrm{~J} / \mathrm{kg}$ and $-1700 \mathrm{~J} / \mathrm{kg}$. To put these values in perspective, field capacity water potential is considered to be around $-10 \mathrm{~J} / \mathrm{kg}$ for sands and $-33 \mathrm{~J} / \mathrm{kg}$ for finer textured soils. Permanent wilt water potential is taken as $-1500 \mathrm{~J} / \mathrm{kg}$. Field capacity is the water potential of the soil a few days after a heavy rain or irrigation. Water at higher potentials typically 

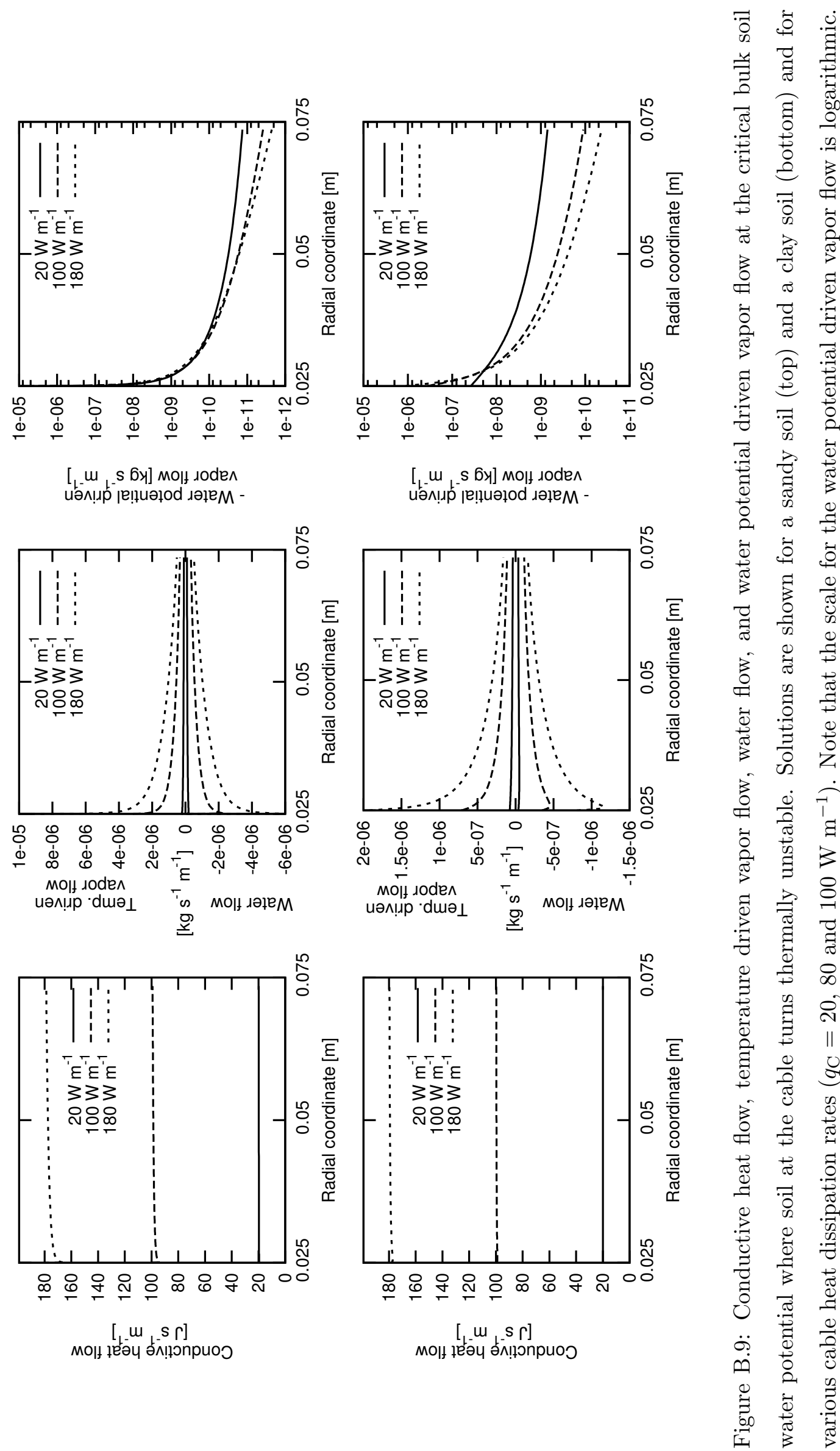

[ $\mathrm{w}$ s $\mathrm{s}$ 6y] Molf toden

[ w s by] MO|f doden

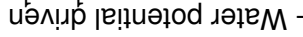

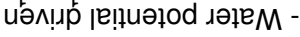
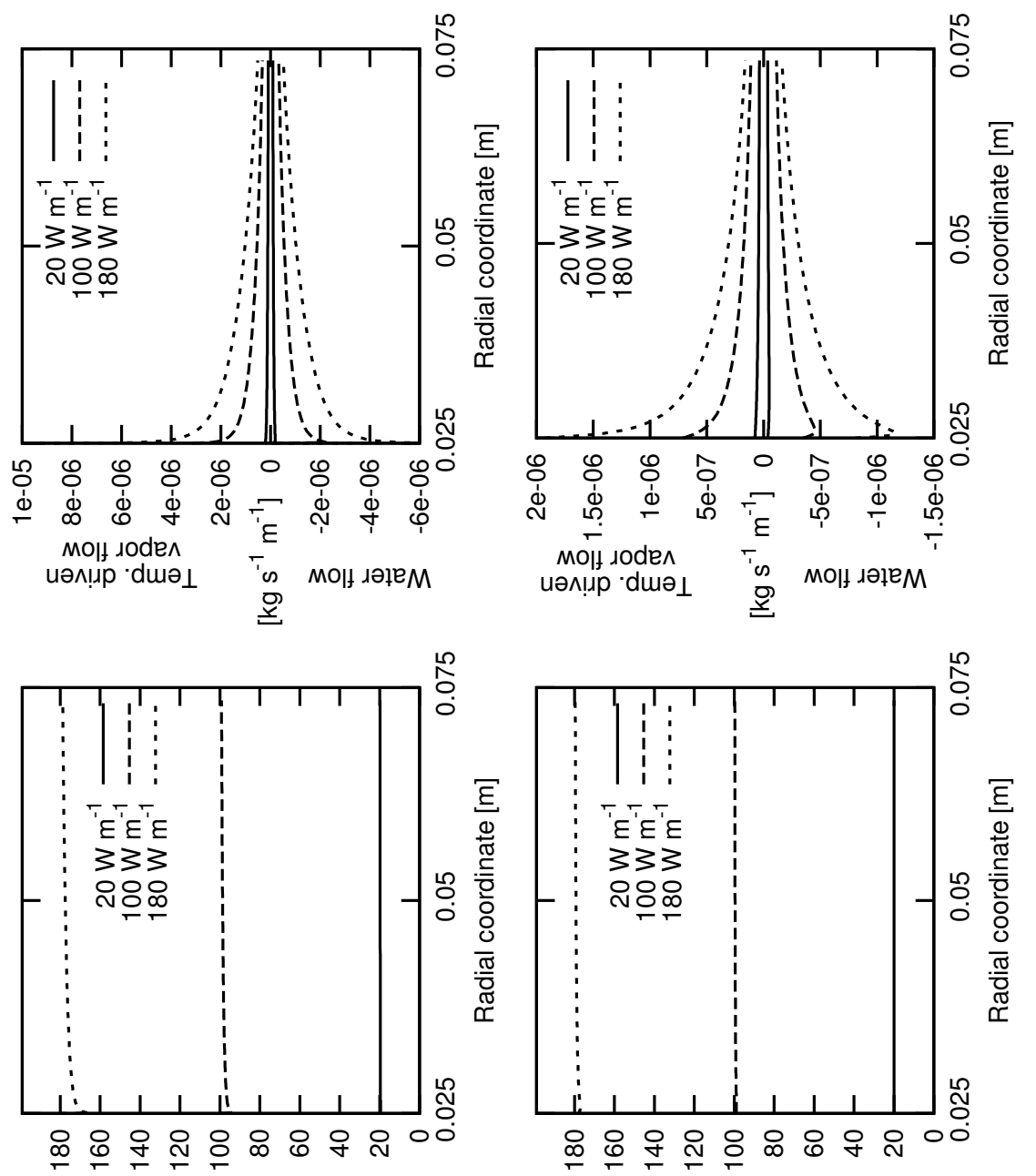

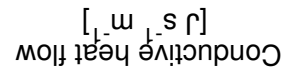

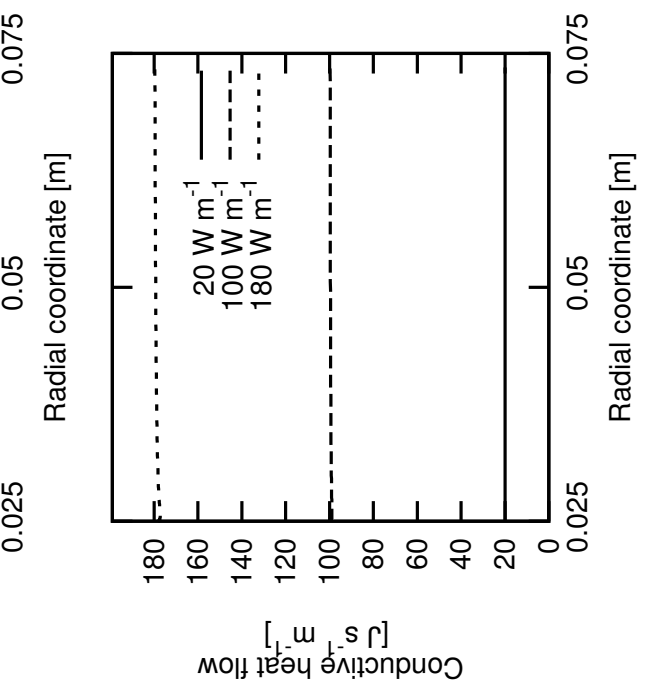

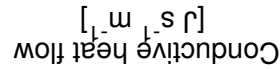



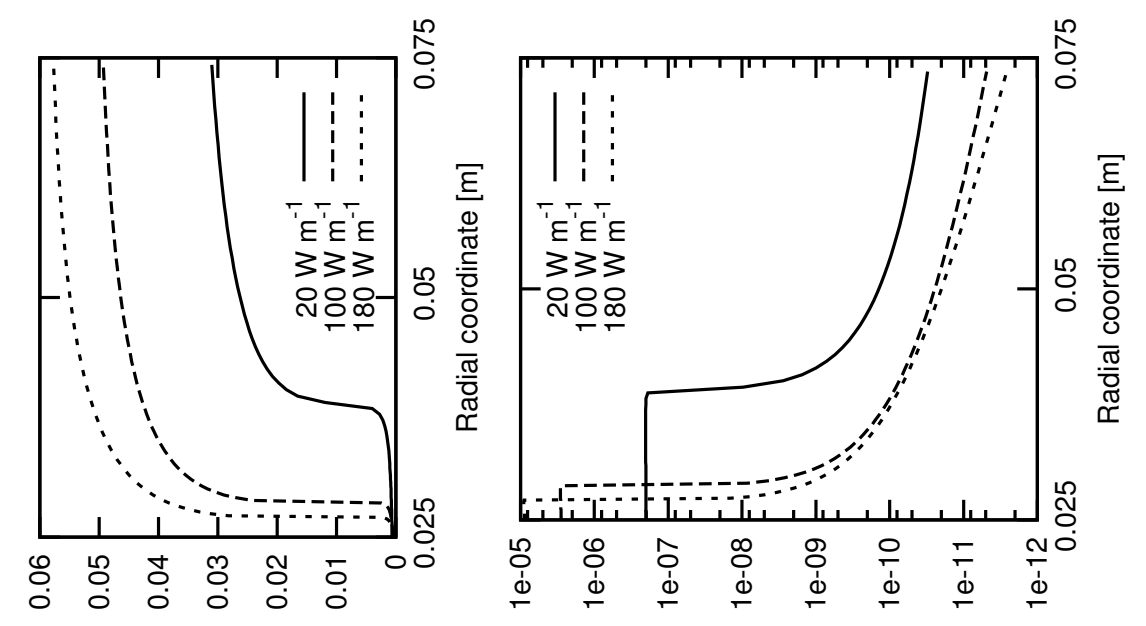

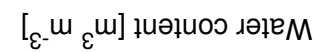

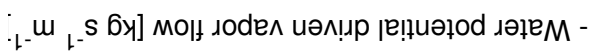
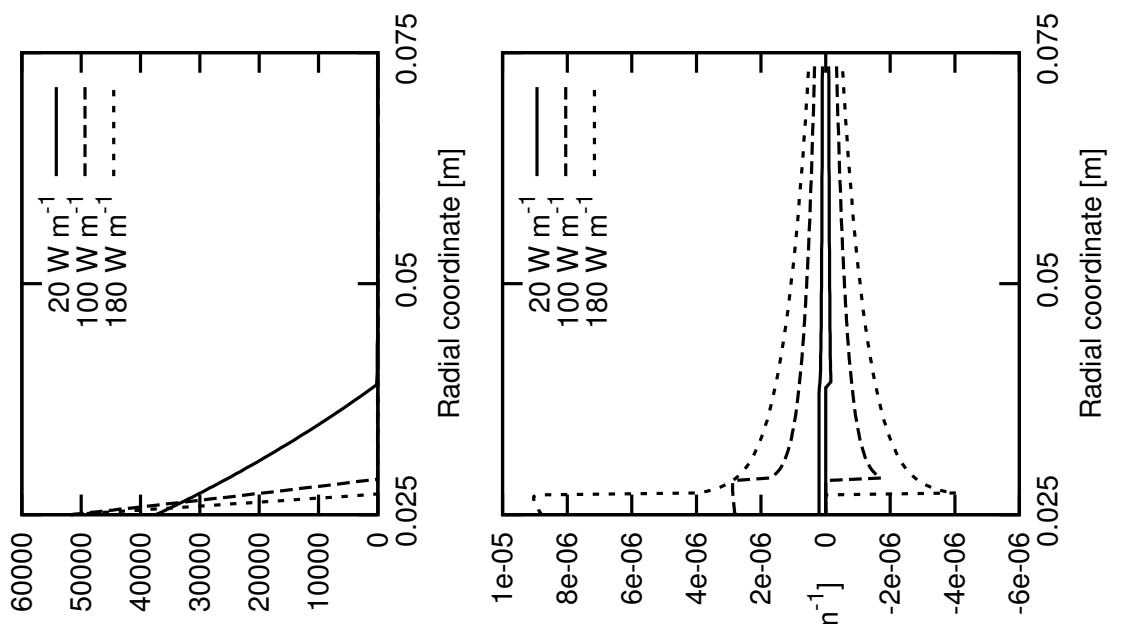

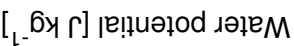

Mo|f Lodes

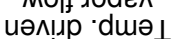

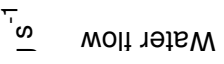

若

范

孛

造鸪

龸言

要

藍

ฮี

芯焉焉

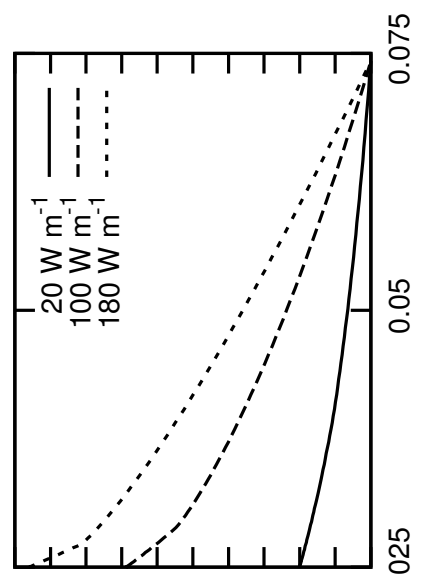

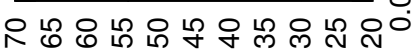

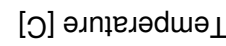

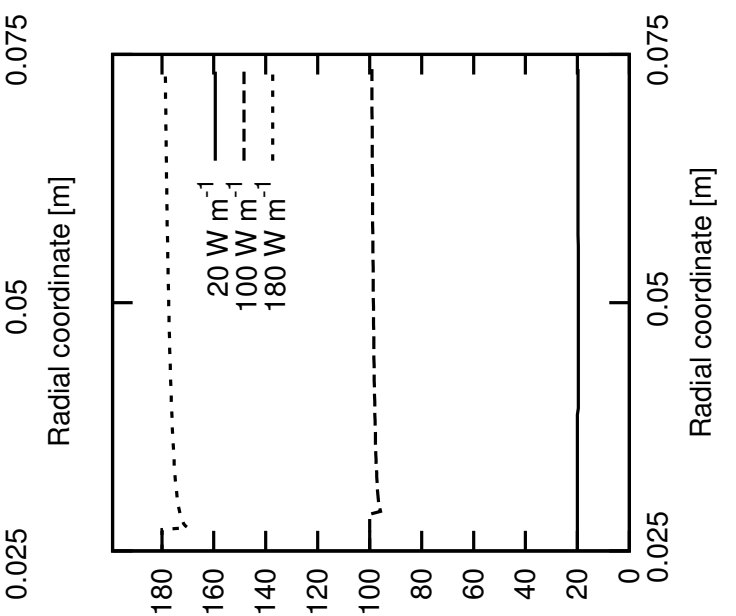

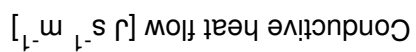



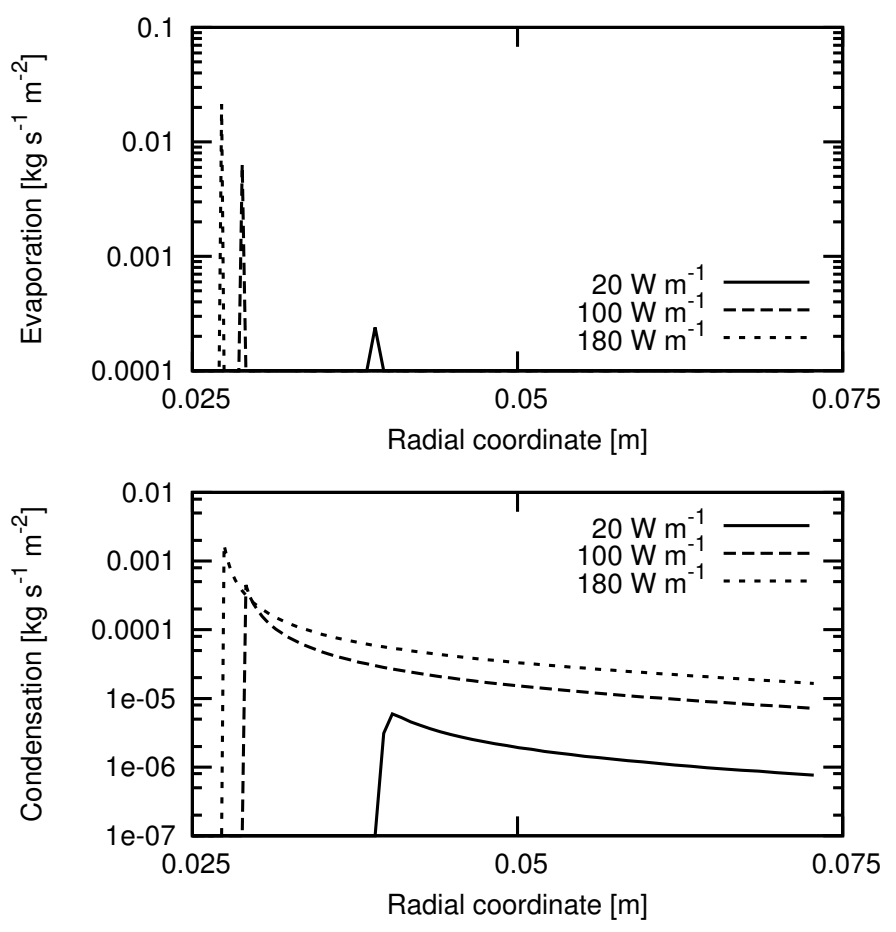

Figure B.11: Evaporation and condensation derived from the solutions presented in Fig. B.10.

drains quickly from the profile. Permanent wilt is the water potential below which plants are no longer able to extract water. The range of plant available water is considered to be between field capacity and permanent wilt. From Fig. B.6 it appears that both soils would stay wet around the cable if the water potential were at field capacity, but all soils would be below critical water potential if the soil dried below the permanent wilt point. Clearly, coarse textured materials are more susceptible to thermal instability than fine textured materials. Not only are coarser soils more susceptible but the instability is also more critical since temperature is higher and water content lower at the point where soil turns thermally unstable.

For designing and selecting the backfill material, as well as choosing the soil in which installing underground cables, fine textured materials are more efficient than coarse materials since they maintain the soil wet and thermally stable for a broader range of water potentials. The high hydraulic conductivity of wet soils allows faster heat dissipation from the cable. Note that soil mechanics have not been analyzed in our study. Swelling clays are prone to shrinking and swelling due to changes in their water content. When temperature changes around the cable and the soil dries out, cracks can form and it can result in air gaps around the cable. This phenomena has a negative effect on heat dissipation, since air has a low 
thermal conductivity.

It would appear that soils near a water table should always be thermally stable, suggesting that in some specific cases it may be advisable to install the cable deeper into the soil profile. Soils from which plant roots have been excluded should also be thermally stable, particularly if they are periodically replenished by rain or irrigation. It appears that soils in which plants are growing would always be susceptible to thermal instability in the investigated range of thermal energy loss from the cable.

\section{B.7 Conclusion}

In our study we presented an analytical estimation for the critical heat dissipation rate from underground electrical power cables at which the soil around the cable dries out. In wet conditions water evaporates at the cable, diffuses as vapor away from the cable, condensates in the dry part and flows back as liquid water. The point where this water-vapor cycle breaks indicates where the soil gets dry and the low thermal conductivity of dry soil has to be used for designing the underground power line system.

Most previous studies about heat dissipation from underground cables did not account for hydraulic dynamics. Our estimation, however, is a powerful and simple tool that considers the complex coupling of hydraulic and thermal dynamics in soils and can be employed in the construction of underground power cables system. 


\section{B.8 Nomenclature}

\begin{tabular}{|c|c|c|}
\hline Symbol & Unit & Description \\
\hline$\beta$ & & empirical parameter $(0.9)$ \\
\hline$\lambda, \lambda_{a}, \lambda_{m}, \lambda_{w}$ & $\mathrm{~W} \mathrm{~m} \mathrm{~m}^{-1} \mathrm{~K}^{-1}$ & thermal conductivity, of air, minerals, water \\
\hline$\theta$ & $\mathrm{m}^{3} \mathrm{~m}^{-3}$ & volumetric water content \\
\hline$\theta_{s}$ & $\mathrm{~m}^{3} \mathrm{~m}^{-3}$ & saturated volumetric water content \\
\hline$\Phi$ & $\mathrm{m}^{3} \mathrm{~m}^{-3}$ & air-filled porosity \\
\hline$\psi$ & $\mathrm{J} \mathrm{kg}^{-1}$ & matric potential \\
\hline$\psi_{e}$ & $\mathrm{~J} \mathrm{~kg}^{-1}$ & air entry potential \\
\hline$b$ & & hydraulic soil parameter \\
\hline$c_{v}^{\prime}$ & $\mathrm{kg} \mathrm{m}^{-3}$ & saturated vapor concentration \\
\hline$D_{0}$ & $\mathrm{~m}^{2} \mathrm{~s}^{-1}$ & binary diffusion coefficient for a gas \\
\hline$D$ & $\mathrm{~m}^{2} \mathrm{~s}^{-1}$ & vapor diffusivity \\
\hline$J$ & $\mathrm{~W} \mathrm{~m}^{-1}$ & electric energy loss of cable \\
\hline$h$ & & relative humidity \\
\hline$k_{a}, k_{m}, k_{w}$ & & weighting factor for air, minerals, water \\
\hline$K$ & $\mathrm{~kg} \mathrm{~s} \mathrm{~m}{ }^{-3}$ & hydraulic conductivity \\
\hline$K_{s}$ & $\mathrm{~kg} \mathrm{~s} \mathrm{~m}^{-3}$ & saturated hydraulic conductivity \\
\hline$L$ & $\mathrm{~J} \mathrm{~kg}^{-1}$ & latent heat of vaporization $\left(2.45 \times 10^{6}\right)$ \\
\hline$m$ & & empirical parameter $(2.3)$ \\
\hline$n$ & & hydraulic soil parameter \\
\hline$M_{w}$ & $\mathrm{~kg} \mathrm{~mol}{ }^{-1}$ & molecular weight of water $(0.018)$ \\
\hline$q_{\mathrm{C}}$ & $\mathrm{J} \mathrm{m}^{-1} \mathrm{~s}^{-1}$ & cable heat dissipation rate \\
\hline$q_{\mathrm{hC}}$ & $\mathrm{J} \mathrm{m}^{-1} \mathrm{~s}^{-1}$ & conductive heat flow \\
\hline$q_{\mathrm{v}}, q_{\mathrm{vP}}, q_{\mathrm{vT}}$ & $\mathrm{kg} \mathrm{m}^{-1} \mathrm{~s}^{-1}$ & vapor flow, driven by water potential, driven by temperatu \\
\hline$q_{\mathrm{lw}}$ & $\mathrm{kg} \mathrm{m}^{-1} \mathrm{~s}^{-1}$ & liquid water flow \\
\hline$R$ & $\mathrm{~J} \mathrm{~mol}^{-1} \mathrm{~K}^{-1}$ & gas constant $(8.31)$ \\
\hline$r_{c}, r_{s}$ & $\mathrm{~m}$ & radius of cable, of bulk soil boundary \\
\hline$s$ & $\mathrm{~kg} \mathrm{~m}^{-3} \mathrm{~K}^{-1}$ & slope of the saturation vapor concentration function \\
\hline$T, T_{c}, T_{s}$ & ${ }^{\circ} \mathrm{C}$ & temperature, at cable, at soil surface \\
\hline$T_{K}$ & $\mathrm{~K}$ & temperature in Kelvin \\
\hline$x_{a}, x_{m}$ & $\mathrm{~m}^{3} \mathrm{~m}^{-3}$ & volumetric fraction of air, solid minerals \\
\hline
\end{tabular}

Table B.2: List of symbols 


\section{References}

Ahmed, M., M. Zarebanadkouki, A. Kaestner, And A. Carminati (2015a): "Measurements of water uptake of maize roots: the key function of lateral roots," Plant and Soil.

Ahmed, M. A., M. Holz, S. K. Woche, J. Bachmann, and A. Carminati (2015b): "Effect of soil drying on mucilage exudation and its water repellency: a new method to collect mucilage," Journal of Plant Nutrition and Soil Science, 178, 821-824.

Ahmed, M. A., E. Kroener, P. Benard, M. Zarebanadkouki, A. Kaestner, and A. Carminati (2015c): "Drying of mucilage causes water repellency in the rhizosphere of maize: measurements and modelling," Plant and Soil, 1-11.

Ahmed, M. A., E. Kroener, M. Holz, M. Zarebanadkouki, and A. Carminati (2014): "Mucilage exudation facilitates root water uptake in dry soils," Functional Plant Biology, 41, 1129-1137.

Albalasmen, A. A. And T. A. Ghezzenei (2014): "Interplay between soil drying and root exudation in rhizosheath development," Plant and Soil, 374, 739-751.

Aravena, J. E., M. Berli, T. A. Ghezzehei, And S. W. Tyler (2010): "Effects of rootinduced compaction on rhizosphere hydraulic properties - x-ray microtomography imaging and numerical simulations," Environmental Science and Technology, 45, 425-431.

Aravena, J. E., M. Berli, S. Ruiz, F. Suárez, T. A. Ghezzehei, and S. W. Tyler (2013): "Quantifying coupled deformation and water flow in the rhizosphere using X-ray microtomography and numerical simulations," Plant and Soil, 1-16.

Axelos, M. AND M. KolB (1990): "Crosslinked biopolymers: Experimental evidence for scalar percolation theory," Physical Review Letters, 64, 1457.

BAlBerg, I. (1987): "Recent developments in continuum percolation," Philosophical Magazine Part B, 56, 991-1003.

Bastian, P., M. Blatt, A. Dedner, C. Engwer, R. Klöfkorn, R. Kornhuber, M. Ohlberger, And O. Sander (2008a): "A Generic Grid Interface for Parallel and Adaptive Scientific Computing. Part II: Implementation and Tests in DUNE," Computing, $82,121-138$. 
Bastian, P., M. Blatt, A. Dedner, C. Engwer, R. Klöfkorn, M. Ohlberger, And O. Sander (2008b): "A Generic Grid Interface for Parallel and Adaptive Scientific Computing. Part I: Abstract Framework," Computing, 82, 103-119.

Bastian, P., F. Heimann, and S. Marnach (2010): "Generic implementation of finite element methods in the Distributed and Unified Numerics Environment (DUNE)," Kybernetika, 46, 294-315.

Bauters, T., T. Steenhuis, D. DiCarlo, J. Nieber, L. Dekker, C. Ritsema, J. ParLANGe, And R. HaVerkamp (2000): "Physics of water repellent soils," Journal of Hydrology, 231, 233-243.

Becker, A. M. AND R. M. ZIFF (2009): "Percolation thresholds on two-dimensional Voronoi networks and Delaunay triangulations," Physical Review E, 80, 041101.

Benard, P., E. Kroener, P. Vontobel, A. Kaestner, and A. Carminati (2015): "Water percolation through the root-soil interface," Advances in Water Resources.

Bengough, A. (2012): "Water dynamics of the root zone: rhizosphere biophysics and its control on soil hydrology," Vadose Zone Journal, 11.

Berkowitz, B. AND I. BAlberg (1992): "Percolation approach to the problem of hydraulic conductivity in porous media," Transport in Porous Media, 9, 275-286.

(1993): "Percolation theory and its application to groundwater hydrology," Water Resources Research, 29, 775-794.

Berkowitz, B. And R. P. Ewing (1998): "Percolation theory and network modeling applications in soil physics," Surveys in Geophysics, 19, 23-72.

Bittelli, M., G. S. Campbell, And F. Tomei (2015): Soil Physics with Python: Transport in the Soil-Plant-Atmosphere System, Oxford University Press, Oxford.

Bittelli, M., F. Ventura, G. S. Campbell, R. L. Snyder, F. Gallegati, and P. R. PISA (2008): "Coupling of heat, water vapor, and liquid water fluxes to compute evaporation in bare soils," Journal of Hydrology, 362, 191-205.

Blatt, M. And P. Bastian (2007a): "The Iterative Solver Template Library," in Applied Parallel Computing. State of the Art in Scientific Computing, ed. by B. Kågström, E. Elmroth, J. Dongarra, and J. Waśniewski, Springer, vol. 4699 of Lecture Notes in Computer Science, 666-675. 
- (2007b): The iterative solver template library, Springer, New York.

Blum, A. (2005): "Drought resistance, water-use efficiency, and yield potentialare they compatible, dissonant, or mutually exclusive?" Crop and Pasture Science, 56, 1159-1168.

Blunt, M. J. (2001): "Flow in porous mediapore-network models and multiphase flow," Current Opinion in Colloid \& Interface Science, 6, 197-207.

Brinker, C. J. AND G. W. Scherer (1990): Sol-gel science: the physics and chemistry of sol-gel processing, Elsevier, Amsterdam.

Brooks, R. H. AND A. T. Corey (1964): "Hydraulic properties of porous media," Hydrology Papers, Colorado State University.

BUCK, A. L. (1981): "New equations for computing vapor pressure and enhancement factor," Journal of Applied Meteorology, 20, 1527-1532.

Campbell, G. (1988): "Soil water potential measurement: An overview," Irrigation Science, 9, 265-273.

Campbell, G. S. (1974): "A simple method for determining unsaturated conductivity from moisture retention data," Soil Science, 117, 311-314.

(1985): Soil physics with BASIC: transport models for soil-plant systems, vol. 14, Elsevier, Amsterdam.

Campbell, G. S. And K. L. Bristow (2006): "Underground Power Cable Installations: Soil Thermal Resistivity," Tech. rep., Decagon Devices.

Campbell, G. S., J. Jungbauer JR, W. Bidlake, and R. Hungerford (1994): "Predicting the effect of temperature on soil thermal conductivity," Soil Science, 158, 307-313.

Campbell, G. S. and J. M. Norman (1998): An introduction to environmental biophysics, second edition, Springer, New York.

Campbell, G. S. and S. Shiozawa (1992): "Prediction of hydraulic properties of soils using particle-size distribution and bulk density data," in Proc. Int. Workshop on Indirect Methods for Estimating the Hydraulic Properties of Unsaturated Soils. University of California, Riverside, 317-328. 
Canova, A., F. Freschi, L. Giaccone, and A. Guerrisi (2012): "The high magnetic coupling passive loop: A steady-state and transient analysis of the thermal behavior," Applied Thermal Engineering, 37, 154-164.

Carminati, A. (2012): "A Model of Root Water Uptake Coupled with Rhizosphere Dynamics," Vadose Zone Journal, 11.

(2013): "Rhizosphere wettability decreases with root age: a problem or a strategy to increase water uptake of young roots?" Frontiers in Plant Science, 4.

Carminati, A., A. Kaestner, H. Flühler, P. Lehmann, D. Or, E. Lehmann, and M. StAMPANONi (2007): "Hydraulic contacts controlling water flow across porous grains," Physical Review E, 76, 026311.

Carminati, A., A. Kaestner, P. Lehmann, And H. Flühler (2008): "Unsaturated water flow across soil aggregate contacts," Advances in water resources, 31, 1221-1232.

Carminati, A., E. Kroener, M. Ahmed, M. Zarebanadkouki, M. Holz, and T. Ghezzenei (in press): "Water for carbon, carbon for water," Vadose Zone Journal, 10, 988-998.

Carminati, A., A. Moradi, D. Vetterlein, P. Vontobel, E. Lehmann, U. Weller, H. Vogel, ANd S. Oswald (2010): "Dynamics of soil water content in the rhizosphere," Plant and Soil, 332, 163-176.

Carminati, A., C. Schneider, A. Moradi, M. Zarebanadkouki, D. Vetterlein, H. Vogel, A. Hildebrandt, U. Weller, L. Schüler, And S. Oswald (2011): "How the Rhizosphere May Favor Water Availability to Roots," Vadose Zone Journal, 10, 988998.

Carminati, A. and D. Vetterlein (2013): "Plasticity of rhizosphere hydraulic properties as a key for efficient utilization of scarce resources," Annals of Botany, 112, 277-290.

Carminati, A., D. Vetterlein, N. Koebernick, S. Blaser, U. Weller, and H.-J. Vogel (2013): "Do roots mind the gap?" Plant and Soil, 367, 651-661.

Carminati, A., D. Vetterlein, U. Weller, H.-J. Vogel, and S. E. Oswald (2009): "When roots lose contact," Vadose Zone Journal, 8, 805-809. 
Chatziathanasiou, V., P. Chatzipanagiotou, i. Papagiannopoulos, G. De Mey, AND B. WiKCEK (2013): "Dynamic thermal analysis of underground medium power cables using thermal impedance, time constant distribution and structure function," Applied Thermal Engineering, 60, 256-260.

Chen, X., L. Hu, Y. Xu, X. CaO, And S. Gubanski (2012): "Investigation of temperature effect on electrical trees in XLPE cable insulation," in Electrical Insulation and Dielectric Phenomena (CEIDP), 2012 Annual Report Conference on, IEEE, 612-615.

Chenu, C. and E. Roberson (1996): "Diffusion of glucose in microbial extracellular polysaccharide as affected by water potential," Soil Biology and Biochemistry, 28, 877884.

Cigre, W. (1998): "21.05, Diagnostic Methods for HV Paper Cables and Accessories," Electra, 60060-3.

Clausnitzer, V. And J. Hopmans (1994): "Simultaneous modeling of transient threedimensional root growth and soil water flow," Plant and Soil, 164, 299-314.

CowAn, I. (1965): "Transport of water in the soil-plant-atmosphere system," Journal of Applied Ecology, 221-239.

Czarnes, S., P. Hallett, A. Bengough, and I. Young (2000): "Root-and microbialderived mucilages affect soil structure and water transport," European Journal of Soil Science, 51, 435-443.

Daly, K. R., S. J. Mooney, M. J. Bennett, N. M. Crout, T. Roose, and S. R. TRACY (2015): "Assessing the influence of the rhizosphere on soil hydraulic properties using X-ray computed tomography and numerical modelling," Journal of experimental botany, 66, 2305-2314.

De Gennes, P.-G. (1976): "On a relation between percolation theory and the elasticity of gels," Journal de Physique Lettres, 37, 1-2.

De Leon, F. And G. J. Anders (2008): "Effects of backfilling on cable ampacity analyzed with the finite element method," Power Delivery, IEEE Transactions on, 23, 537-543.

de Lieto Vollaro, R., L. Fontana, A. Quintino, and A. Vallati (2011a): "Improving evaluation of the heat losses from arrays of pipes or electric cables buried in homogeneous soil," Applied Thermal Engineering, 31, 3768-3773. 
de Lieto Vollaro, R., L. Fontana, and A. Vallati (2011b): "Thermal analysis of underground electrical power cables buried in non-homogeneous soils," Applied Thermal Engineering, 31, 772-778.

(2014): "Experimental study of thermal field deriving from an underground electrical power cable buried in non-homogeneous soils," Applied Thermal Engineering, 62, 390-397.

DE VRIEs, D. (1963): "Thermal properties of soils," Physics of Plant Environment, 1, 57-109.

Deery, D. M., J. B. Passioura, J. R. Condon, And A. Katupitiya (2013): "Uptake of water from a Kandosol subsoil. II. Control of water uptake by roots," Plant and Soil, 368(1-2), 649-667.

Del Gado, E., L. de Arcangelis, and A. Coniglio (1998): "A percolation dynamic approach to the sol-gel transition," Journal of Physics A: Mathematical and General, 31, 1901.

Deng, W., P. D. Hallett, D.-S. Jeng, G. R. Squire, P. E. Toorop, and P. P. IANNETta (2015): "The effect of natural seed coatings of Capsella bursa-pastoris L. Medik.(shepherds purse) on soil-water retention, stability and hydraulic conductivity," Plant and Soil, 387, 167-176.

Densley, J. (2001): "Ageing mechanisms and diagnostics for power cables-an overview," Electrical Insulation Magazine, IEEE, 17, 14-22.

Dirmeyer, P. A., X. GaO, M. Zhao, Z. Guo, T. Oki, and N. Hanasaki (2006): "GSWP-2: Multimodel analysis and implications for our perception of the land surface," Bulletin of the American Meteorological Society, 87, 1381-1397.

Doussan, C., A. Pierret, E. Garrigues, and L. Pagès (2006): "Water uptake by plant roots: II-Modelling of water transfer in the soil root-system with explicit account of flow within the root system-Comparison with experiments," Plant and soil, 283, 99-117.

Draye, X., Y. Kim, G. Lobet, and M. Javaux (2010): "Model-assisted integration of physiological and environmental constraints affecting the dynamic and spatial patterns of root water uptake from soils," Journal of Experimental Botany, 61, 2145-2155.

Dunbabin, V. M., J. A. Postma, A. Schnepf, L. Pagès, M. Javaux, L. Wu, D. Leitner, Y. L. Chen, Z. Rengel, And A. J. Diggle (2013): "Modelling root-soil inter- 
actions using three-dimensional models of root growth, architecture and function," Plant and Soil, 372, 93-124.

DUNE (2014): "Distributed and Unified Numerics Environment," Url: http://www. dune-project.org.

Ebrahimi, A. N. And D. Or (2014): "Microbial dispersal in unsaturated porous media: Characteristics of motile bacterial cell motions in unsaturated angular pore networks," Water Resources Research.

Feddes, R. A., P. Kowalik, K. Kolinska-Malinka, and H. Zaradny (1976): "Simulation of field water uptake by plants using a soil water dependent root extraction function," Journal of Hydrology, 31, 13-26.

Flory, P. J. (1953): Principles of polymer chemistry, Cornell University Press.

Frensch, J. And E. Steudle (1989): "Axial and radial hydraulic resistance to roots of maize (Zea mays L.)," Plant Physiology, 91, 719-726.

GaO, Y., Y. Yang, W. Ling, H. Kong, And X. Zhu (2011): "Gradient distribution of root exudates and polycyclic aromatic hydrocarbons in rhizosphere soil," Soil Science Society of America Journal, 75, 1694-1703.

Gardner, W. (1960): "Dynamic aspects of water availability to plants," Soil Science, 89, $63-73$.

Gefen, Y., W.-H. Shin, R. B. Laibowitz, and J. Viggiano (1986): "Nonlinear behavior near the percolation metal-insulator transition," Physical Review Letters, 57, 3097.

Gerke, H. And M. V. Genuchten (1993): "A dual-porosity model for simulating the preferential movement of water and solutes in structured porous media," Water Resources Research, 29, 305-319.

Geuzaine, C. And J.-F. Remacle (2009): "Gmsh: A 3-D finite element mesh generator with built-in pre-and post-processing facilities," International Journal for Numerical Methods in Engineering, 79, 1309-1331.

Ghanbarzadeh, S., M. Prodanović, and M. A. Hesse (2014): "Percolation and Grain Boundary Wetting in Anisotropic Texturally Equilibrated Pore Networks," Physical Review Letters, 113, 048001. 
Goebel, M.-O., S. K. Woche, P. M. Abraham, G. E. Schaumann, and J. BachMANN (2013): "Water repellency enhances the deposition of negatively charged hydrophilic colloids in a water-saturated sand matrix," Colloids and Surfaces A: Physicochemical and Engineering Aspects, 431, 150-160.

Gregory, P. (2006): "Roots, rhizosphere and soil: the route to a better understanding of soil science?" European Journal of Soil Science, 57, 2-12.

Griend, A. A. AND M. Owe (1994): "Bare soil surface resistance to evaporation by vapor diffusion under semiarid conditions," Water Resources Research, 30, 181-188.

Guinel, F. And M. MCCully (1986): "Some water-related physical properties of maize root-cap mucilage," Plant, Cell \& Environment, 9, 657-666.

Hallett, P., D. Gordon, And A. Bengough (2003): "Plant influence on rhizosphere hydraulic properties: direct measurements using a miniaturized infiltrometer," New Phytologist, 157, 597-603.

Hartley, J. AND W. Black (1981): "Transient simultaneous heat and mass transfer in moist, unsaturated soils," Journal of Heat Transfer, 103, 376-382.

Hassanein, R., E. Lehmann, and P. Vontobel (2005): "Methods of scattering corrections for quantitative neutron radiography," Nuclear Instruments and Methods in Physics Research Section A: Accelerators, Spectrometers, Detectors and Associated Equipment, 542, 353-360.

Hinsinger, P., A. Bengough, D. Vetterlein, And I. Young (2009): "Rhizosphere: biophysics, biogeochemistry and ecological relevance," Plant and Soil, 321, 117-152.

Huber, K., J. Vanderborght, M. Javaux, N. Schröder, I. C. Dodd, And H. VEREECKEN (2014): "Modelling the impact of heterogeneous rootzone water distribution on the regulation of transpiration by hormone transport and/or hydraulic pressures," Plant and Soil, 384, 93-112.

Hunt, A. (2001): "Applications of percolation theory to porous media with distributed local conductances," Advances in Water Resources, 24, 279-307.

(2004): "Continuum percolation theory for water retention and hydraulic conductivity of fractal soils: Estimation of the critical volume fraction for percolation," Advances in Water Resources, 27, 175-183. 
Islam, M., S. Mekhilef, ANd R. SAidur (2013): "Progress and recent trends of wind energy technology," Renewable and Sustainable Energy Reviews, 21, 456-468.

JARVIS, N. (1989): "A simple empirical model of root water uptake," Journal of Hydrology, $107,57-72$.

Javaux, M., T. Schröder, J. Vanderborght, and H. Vereecken (2008): "Use of a three-dimensional detailed modeling approach for predicting root water uptake," Vadose Zone Journal, 7, 1079-1088.

JoEkAR-NiAsar, V. AND S. HASSANIZADEH (2012): "Analysis of fundamentals of two-phase flow in porous media using dynamic pore-network models: A review," Critical Reviews in Environmental Science and Technology, 42, 1895-1976.

Jones, C. I. And M. C. McManus (2010): "Life-cycle assessment of $11 \mathrm{kV}$ electrical overhead lines and underground cables," Journal of Cleaner Production, 18, 1464-1477.

Kaestner, A., S. Hartmann, G. Kühne, G. Frei, C. Grünzweig, L. Josic, F. Schmid, And E. Lehmann (2011): "The ICON beamline-a facility for cold neutron imaging at SINQ," Nuclear Instruments and Methods in Physics Research Section A: Accelerators, Spectrometers, Detectors and Associated Equipment, 659, 387-393.

KELLOW, M. (1981): "A numerical procedure for the calculation of the temperature rise and ampacity of underground cables," Power Apparatus and Systems, IEEE Transactions on, $3322-3330$.

Keyes, S. D., K. R. Daly, N. J. Gostling, D. L. Jones, P. Talboys, B. R. Pinzer, R. Boardman, I. Sinclair, A. Marchant, and T. Roose (2013): "High resolution synchrotron imaging of wheat root hairs growing in soil and image based modelling of phosphate uptake," New Phytologist, 198, 1023-1029.

Klute, A., C. Dirksen, et Al. (2003): "Hydraulic conductivity and diffusivity: Laboratory methods," SOIL SCIENCE SOCIETY OF AMERICA BOOK SERIES, 687-734.

Knipfer, T. And W. Fricke (2010): "Root pressure and a solute reflection coefficient close to unity exclude a purely apoplastic pathway of radial water transport in barley (Hordeum vulgare)," New Phytologist, 187, 159-170.

Kovac, N., N. Grulovic-Pavluanic, And A. Kukavica (2013): "Generated heat within power cable sheaths per unit time and volume," Applied Thermal Engineering, 52, 90-96. 
Kroener, E., M. A. Ahmed, And A. Carminati (2015): "Roots at the percolation threshold," Physical Review E, 91, 042706.

Kroener, E., G. S. Campbell, and M. Bittelli (in preparation): "Estimation of thermal instabilities in soils for heat dissipation of underground electrical power cables," .

Kroener, E., A. Vallati, and M. Bittelli (2014a): "Numerical simulation of coupled heat, liquid water and water vapor in soils for heat dissipation of underground electrical power cables," Applied Thermal Engineering, 70, 510 - 523.

Kroener, E., M. Zarebanadkouki, M. Bittelli, and A. Carminati (submitted 2016): "Simulation of root water uptake under consideration of non-equilibrium dynamics in the rhizosphere," submitted to Water Resources Research.

Kroener, E., M. Zarebanadkouki, A. Kaestner, And A. Carminati (2014b): "Nonequilibrium water dynamics in the rhizosphere: How mucilage affects water flow in soils," Water Resources Research, 50, 6479-6495.

KrÖNER, A., E. KrÖNER, AND H. KrÖNER (submitted 2015): "Numerical approximation of level set power mean curvature flow," submitted to SIAM Journal on Numerical Analysis.

LARsson, M. AND N. JaRvis (1999): "Evaluation of a dual-porosity model to predict fieldscale solute transport in a macroporous soil," Journal of Hydrology, 215, 153-171.

Leitner, D., A. Schnepf, S. Klepsch, and T. Roose (2010): "Comparison of nutrient uptake between three-dimensional simulation and an averaged root system model," Plant Biosystems, 144, 443-447.

Li, M., T. Shinano, and T. Tadano (1997): "Distribution of exudates of lupin roots in the rhizosphere under phosphorus deficient conditions," Soil Science and Plant Nutrition, $43,237-245$.

Lin, K.-Y., J. R. DANiel, And R. L. Whistler (1994): "Structure of chia seed polysaccharide exudate," Carbohydrate polymers, 23, 13-18.

Liu, B.-B., E. Steudle, X.-P. Deng, and S.-Q. Zhang (2009): "Root pressure probe can be used to measure the hydraulic properties of whole root systems of corn (Zea mays L.)," Botanical Studies (Taipei, Taiwan), 50, 303-310. 
Lobet, G., V. Couvreur, F. Meunier, M. Javaux, And X. Draye (2014): "Plant water uptake in drying soils," Plant physiology, 164, 1619-1627.

Lorenz, C. D. And R. M. ZIFF (1998): "Precise determination of the bond percolation thresholds and finite-size scaling corrections for the sc, fcc, and bcc lattices," Physical Review E, 57, 230.

LYNCH, J. P. (2013): "Steep, cheap and deep: an ideotype to optimize water and N acquisition by maize root systems," Annals of Botany, 112, 347-357.

LyNCH, J. P. AND K. M. BROWn (2012): "New roots for agriculture: exploiting the root phenome," Philosophical Transactions of the Royal Society B: Biological Sciences, 367, $1598-1604$.

LyNCH, J. P. AND T. WOJCIEChOWski (2015): "Opportunities and challenges in the subsoil: pathways to deeper rooted crops," Journal of Experimental Botany, eru508.

Marshall, J. S., P. D. Hines, J. D. Zhang, F. Minervini, and S. Rinjitham (2013): "Modeling the impact of electric vehicle charging on heat transfer around underground cables," Electric Power Systems Research, 97, 76-83.

MA'Shum, M. AND V. FARMER (1985): "Origin and assessment of water repellency of a sandy South Australian soil," Soil Research, 23, 623-626.

Maurel, C., L. Verdoucq, D.-T. Luu, and V. Santoni (2008): "Plant aquaporins: membrane channels with multiple integrated functions," Annu. Rev. Plant Biol., 59, 595624.

McCully, M. (1995): "How Do Real Roots Work?(Some New Views of Root Structure)." Plant physiology, 109, 1.

MCCully, M. AND J. Boyer (1997): "The expansion of maize root-cap mucilage during hydration. 3. Changes in water potential and water content," Physiologia Plantarum, 99, 169-177.

McInNEs, K. (1981): "Thermal conductivities of soils from dryland wheat regions in Eastern Washington," Ph.D. thesis, Washington State University.

Meir, Y. (1999): "Percolation-type description of the metal-insulator transition in two dimensions," Physical Review Letters, 83, 3506. 
Minopoli, D. (2007/2008): "Analisi sperimentale del campo termico nell'intorno di un cavidotto interrato per valutare i limiti di sicurezza," Ph.D. thesis, Facoltà di ingegneria Sapienza Roma.

Moradi, A., A. Carminati, D. Vetterlein, P. Vontobel, E. Lehmann, U. Weller, J. Hopmans, H. Vogel, And S. Oswald (2011): "Three-dimensional visualization and quantification of water content in the rhizosphere," New Phytologist.

Moradi, A. B., A. Carminati, A. Lamparter, S. K. Woche, J. Bachmann, D. VetTERLEin, H.-J. Vogel, AND S. E. Oswald (2012): "Is the rhizosphere temporarily water repellent?" Vadose Zone Journal, 11.

Moradi, A. B., H. M. Conesa, B. Robinson, E. Lehmann, G. Kuehne, A. Kaestner, S. Oswald, AND R. Schulin (2009): "Neutron radiography as a tool for revealing root development in soil: capabilities and limitations," Plant and Soil, 318, 243-255.

Morris, E., A. Cutler, S. Ross-Murphy, D. Rees, And J. Price (1981): "Concentration and shear rate dependence of viscosity in random coil polysaccharide solutions," Carbohydrate Polymers, 1, 5-21.

Muñoz, L., A. Cobos, O. Diaz, And J. Aguilera (2012): "Chia seeds: Microstructure, mucilage extraction and hydration," Journal of Food Engineering, 108, 216-224.

Neher, J. AND M. MCGRath (Oct. 1957): "The calculation of the temperature rise and load capability of cable systems," AIEE Transactions, Part 3, 752-772.

North, G. B. And P. S. Nobel (1997): "Drought-induced changes in soil contact and hydraulic conductivity for roots of Opuntia ficus-indica with and without rhizosheaths," Plant and Soil, 191, 249-258.

OcŁoń, P., P. Cisek, M. Pilarczyk, and D. Taler (2015a): "Numerical simulation of heat dissipation processes in underground power cable system situated in thermal backfill and buried in a multilayered soil," Energy Conversion and Management, 95, 352-370.

OcŁoń, P., P. Cisek, D. Taler, M. Pilarczyk, and T. Szwarc (2015b): "Optimizing of the underground power cable bedding using momentum-type particle swarm optimization method," Energy. 
Or, D., S. Phutane, And A. Dechesne (2007): "Extracellular polymeric substances affecting pore-scale hydrologic conditions for bacterial activity in unsaturated soils," Vadose Zone Journal, 6, 298-305.

Oswald, S. E., M. Menon, A. Carminati, P. Vontobel, E. Lehmann, And R. Schulin (2008): "Quantitative imaging of infiltration, root growth, and root water uptake via neutron radiography," Vadose Zone Journal, 7, 1035-1047.

Papagiannopoulos, I., V. Chatziathanasiou, L. Exizidis, G. Andreou, G. De Mey, AND B. WiKceK (2013): "Behaviour of the thermal impedance of buried power cables," International Journal of Electrical Power 83 Energy Systems, 44, 383-387.

PAssioura, J. B. (1980): "The transport of water from soil to shoot in wheat seedlings," Journal of Experimental Botany, 31, 333-345.

PDELAB (2014): Url: http://www.dune-project.org/pdelab/index.html.

Penman, H. (1940): "Gas and vapour movements in the soil: I. The diffusion of vapours through porous solids," The Journal of Agricultural Science, 30, 437-462.

Pike, G. And C. Seager (1974): "Percolation and conductivity: A computer study. I," Physical Review B, 10, 1421.

Pleinert, H. and E. Lehmann (1997): "Determination of hydrogenous distributions by neutron transmission analysis," Physica B: Condensed Matter, 234, 1030-1032.

Public Survice Commission of Wisconsin (2011): "Underground Electric Transmission Lines," Url: psc.wi.gov/thelibrary/publications/electric/electric11.pdf.

RAIKH, M. AND I. RUZIN (1990): "Size effect in the longitudinal hopping conduction of a narrow two-dimensional channel," Physical Review B, 42, 11203.

Ranathunge, K., L. Kotula, E. Steudle, and R. Lafitte (2004): "Water permeability and reflection coefficient of the outer part of young rice roots are differently affected by closure of water channels (aquaporins) or blockage of apoplastic pores," Journal of Experimental Botany, 55, 433-447.

Read, D., A. Bengough, P. Gregory, J. Crawford, D. Robinson, C. Scrimgeour, I. Young, K. Zhang, And X. Zhang (2003): "Plant roots release phospholipid surfactants that modify the physical and chemical properties of soil," New Phytologist, 157, 315-326. 
Read, D. And P. Gregory (1997): "Surface tension and viscosity of axenic maize and lupin root mucilages," New phytologist, 137, 623-628.

Richards, L. A. (1931): "Capillary conduction of liquids through porous mediums," Journal of Applied Physics, 1, 318-333.

Roose, T. AND A. Fowler (2004): "A mathematical model for water and nutrient uptake by plant root systems," Journal of Theoretical Biology, 228, 173-184.

Saito, H., J. Simunek, And B. P. Mohanty (2006): "Numerical analysis of coupled water, vapor, and heat transport in the vadose zone," Vadose Zone Journal, 5, 784-800.

Schmidt, S., A. Bengough, P. Gregory, D. V. Grinev, and W. Otten (2012): "Estimating root-soil contact from 3D X-ray microtomographs," European Journal of Soil Science, 63, 776-786.

Seager, C. And G. Pike (1974): "Percolation and conductivity: A computer study. II," Physical Review B, 10, 1435.

Sposito, G. (2013): "Green water and global food security," Vadose Zone Journal, 12.

Stauffer, D. (1985): Introduction to percolation theory, Taylor \& Francis Ltd.

Steduto, P., J.-M. Faurès, J. Hoogeveen, J. Winpenny, And J. Burke (2012): Coping with water scarcity: an action framework for agriculture and food security, Food and Agriculture Organization of the United Nations Rome, Italy.

Steenhuis, T. S., A. G. Hunt, J.-Y. Parlange, and R. P. Ewing (2005): "Assessment of the application of percolation theory to a water repellent soil," Soil Research, 43, 357360.

Steudle, E. (2000): "Water uptake by plant roots: an integration of views," Plant and Soil, $226,45-56$.

Steudle, E., R. Oren, And E.-D. Schulze (1987): "Water transport in maize roots measurement of hydraulic conductivity, solute permeability, and of reflection coefficients of excised roots using the root pressure probe," Plant Physiology, 84, 1220-1232.

Taylor, S. A. And L. Cavazza (1954): "The movement of soil moisture in response to temperature gradients," Soil Science Society of America Journal, 18, 351-358. 
Thullner, M. And P. Baveye (2008): "Computational pore network modeling of the influence of biofilm permeability on bioclogging in porous media," Biotechnology and Bioengineering, 99, 1337-1351.

Tron, S., G. Bodner, F. Laio, L. Ridolfi, and D. Leitner (2015): "Can diversity in root architecture explain plant water use efficiency? A modeling study," Ecological modelling, 312, 200-210.

Valvatne, P. H. And M. J. Blunt (2004): "Predictive pore-scale modeling of two-phase flow in mixed wet media," Water Resources Research, 40.

Vandevivere, P. And P. Baveye (1992): "Effect of bacterial extracellular polymers on the saturated hydraulic conductivity of sand columns." Applied and Environmental Microbiology, 58, 1690-1698.

Vogel, H.-J. And K. Roth (2001): "Quantitative morphology and network representation of soil pore structure," Advances in water resources, 24, 233-242.

Wasson, A., R. Richards, R. Chatrath, S. Misra, S. S. Prasad, G. Rebetzke, J. KirkegaArd, J. Christopher, And M. WATt (2012): "Traits and selection strategies to improve root systems and water uptake in water-limited wheat crops," Journal of Experimental Botany, 63, 3485-3498.

Watt, M., M. McCully, And C. Jeffree (1993): "Plant and bacterial mucilages of the maize rhizosphere: comparison of their soil binding properties and histochemistry in a model system," Plant and Soil, 151, 151-165.

Watt, M., M. E. McCully, And M. J. Canny (1994): "Formation and stabilization of rhizosheaths of Zea mays L.(Effect of soil water content)," Plant Physiology, 106, 179-186.

Watt, M., W. K. Silk, And J. B. Passioura (2006): "Rates of root and organism growth, soil conditions, and temporal and spatial development of the rhizosphere," Annals of Botany, 97, 839-855.

YounG, I. (1995): "Variation in moisture contents between bulk soil and the rhizosheath of wheat (Triticum aestivum L. cv. Wembley)," New Phytologist, 130, 135-139.

Zarebanadkouki, M., M. A. Ahmed, And A. Carminati (2015): "Hydraulic conductivity of the root-soil interface of lupin in sandy soil after drying and rewetting," Plant and Soil, 1-14. 
Zarebanadkouki, M. And A. Carminati (2014): "Reduced root water uptake after drying and rewetting," Journal of Plant Nutrition and Soil Science, 177, 227-236.

Zarebanadkouki, M., Y. X. Kim, And A. Carminati (2013): "Where do roots take up water? Neutron radiography of water flow into the roots of transpiring plants growing in soil," New Phytologist. 


\section{Lebenslauf}

\section{Name: Eva Kröner}

Geboren: 21.08.1987 in Aachen, Deutschland

\section{Ausbildung}

2006 Abitur an der Heimschule St. Landolin in Ettenheim, Deutschland

Okt. 2006 Diplomstudium in Physik an der Ruprecht-Karls-Universität

- Jul. 2012 Heidelberg, Deutschland

Okt. 2012 Doktorandin in der Abteilung Bodenhydrologie der Fakultät für Agrar-

- Feb. 2016 wissenschaften an der Georg-August Universität Göttingen, Deutschland 



\section{Declarations}

1. Hiermit erkläre ich, dass diese Arbeit weder in gleicher noch in ähnlicher Form bereits anderen Prüfungsbehörden vorgelegen hat. Weiter erkläre ich, dass ich mich an keiner anderen Hochschule um einen Doktorgrad beworben habe.

Göttingen, den 5. Januar 2016

(Eva Kröner)

2. Hiermit erkläre ich eidesstattlich, dass diese Dissertation selbständig und ohne unerlaubte Hilfe angefertigt wurde.

Göttingen, den 5. Januar 2016

(Eva Kröner) 
